2. To: (Receiving Organization)

Distribution

5. Proj./Prog./Dept./Div.:

TWRS

8. Originator Remarks:

For approval and release.

11. Receiver Remarks

11A. Design Baseline Document?

[] Yes

[X] No
3. From: (originating organization)

W. L. Will is, NHC

6. Design Authority/ Design Agent/Cog. Engr.:

W. L. Willis

\section{Related EDT No.:}

NA

7. Purchase order No.:

NA

9. Equip./Component No.:

NA

10. System/8ldg./Facility: NA

12. Major Assm. Dwg. No.:

NA

13. Permit/Permit Application No.: NA

14. Required Response Date:

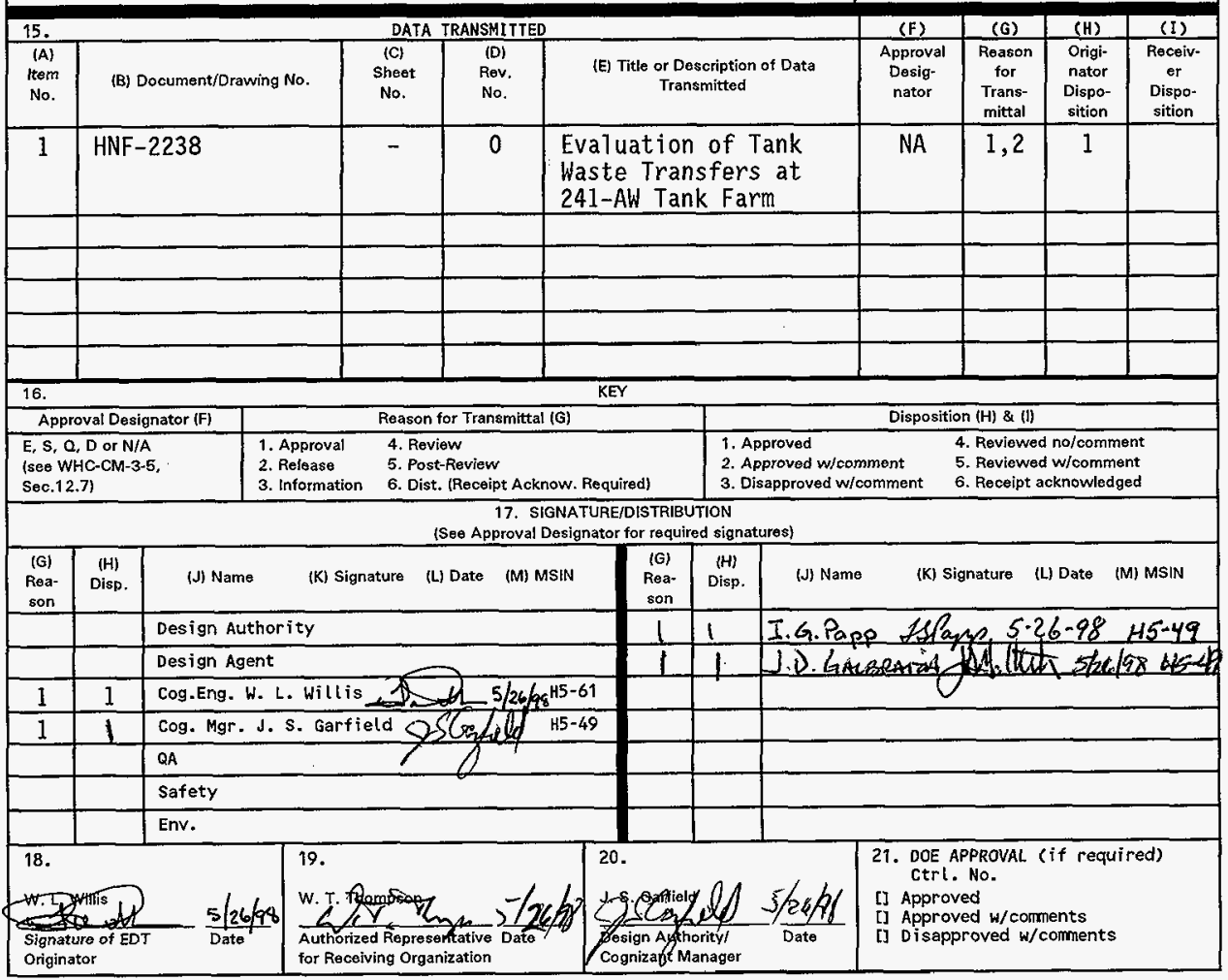

BD-7400-172-2 (05/96) GEF097 
HNF-2238, Rev. 0

\section{Evaluation of Tank Waste Transfers at 241-AW Tank Farm}

W. L. Willis, W. A. Peiffer (B\&W), B. B. Peters (MACTEC), and

T. L. Waldo II (Cogema)

Numatec Hanford Corporation, Richland, WA 99352

U.S. Department of Energy Contract DE-AC06-96RL13200

EDT/ECN: EDT 622714

Org Code: $8 \mathrm{C} 451$

B\&R Code: EW3130010

UC: 721

Charge Code: D2713

Tota1 Pages: 205

Key Words: 241-AW, evaluation, privatization, TWRS

Abstract: The purpose of this evaluation is to determine if existing or $\mathrm{pl}$ anned equipment and systems are capable of supporting the

Privatization Mission of the Tank Farms and continuing operations

through the end of Phase 1B Privatization Mission.

TRADEMARK DISCLAIMER. Reference herein to any specific commercial product, process, or service by trade name, trademark, manufacturer, or otherwise, does not necessarily constitute or imply its endorsement, recommendation, or favoring by the United States Government or any agency thereof or $i$ ts contractors or subcontractors.

Printed in the United States of America. To obtain copies of this document, contact: Document control Services, P.O. Box 950, Mailstop H6-08, Richland WA 99352, Phone (509) 372-2420; Fax (509) 376-4989.

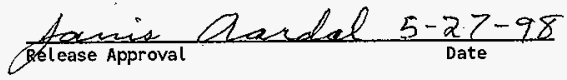

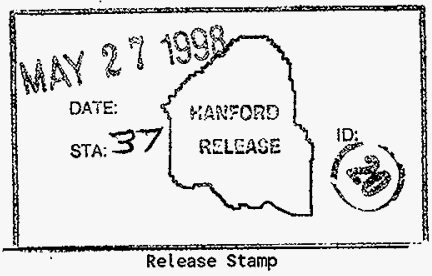


HNF-2238

Revision 0

\title{
EVALUATION OF TANK WASTE TRANSFERS AT 241-AW TANK FARM
}

May 1998

\author{
W. L. Willis \\ Numatec Hanford Corporation \\ Richland, Washington \\ W. A. Peiffer \\ B\&W Hanford Company \\ Richland, Washington \\ B. B. Peters \\ MACTEC \\ Richland, Washington \\ T. L. Waldo II \\ SGN Eurisys Services Company \\ Richland, Washington
}

Prepared for

U.S. Department of Energy

Richland, Washington 
HNF-2238

Revision 0

This page intentionally left blank. 
HNF-2238

Revision 0

CONTENTS

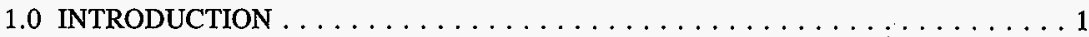

1.1 PURPOSE OF EVALUATION $\ldots \ldots \ldots \ldots \ldots \ldots \ldots \ldots \ldots \ldots$

1.2 BACKGROUND INFORMATION $\ldots \ldots \ldots \ldots \ldots \ldots \ldots \ldots \ldots$

1.3 DOCUMENT ORGANIZATION $\ldots \ldots \ldots \ldots \ldots \ldots \ldots \ldots \ldots$

1.3.1 Road Map to Appendices $\ldots \ldots \ldots \ldots \ldots \ldots \ldots$

2.0 PROCESS AND EQUIPMENT EXPECTATIONS $\ldots \ldots \ldots \ldots \ldots \ldots \ldots$

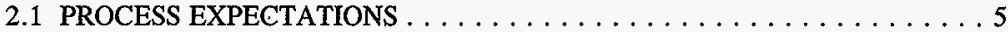

2.2 EQUIPMENT EXPECTATIONS $\ldots \ldots \ldots \ldots \ldots \ldots \ldots \ldots \ldots \ldots$

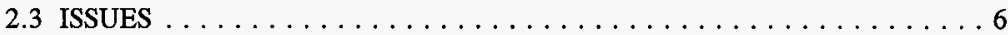

3.0 CONCLUSIONS AND RECOMMENDATIONS $\ldots \ldots \ldots \ldots \ldots \ldots$

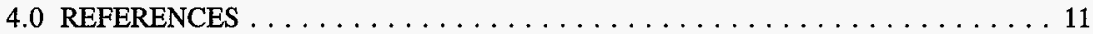

APPENDIX

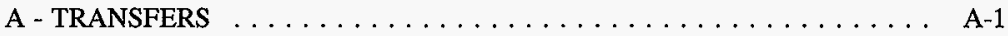

B - TRANSFER ISSUES $\ldots \ldots \ldots \ldots \ldots \ldots \ldots \ldots \ldots$ B-1

C - TRANSFER REQUIREMENTS AND EXPECTATIONS $\ldots \ldots \ldots \ldots \ldots$ C-1

D - CALCULATIONS $\ldots \ldots \ldots \ldots \ldots \ldots \ldots \ldots \ldots \ldots \ldots \ldots \ldots \ldots \ldots \ldots$

E - 241-AW PROJECT REQUIREMENTS AND BASIS $\ldots \ldots \ldots \ldots \ldots \ldots$ E-1 
HNF-2238

Revision 0

\section{LIST OF TERMS}

$\begin{array}{ll}\text { ALARA } & \text { As-low-as-reasonably-achievable } \\ \text { BIO } & \text { Basis for Interim Operation } \\ \text { CAS } & \text { Condition Assessment Survey } \\ \text { DOE } & \text { U.S. Department of Energy } \\ \text { DST } & \text { Double-shell tank } \\ \text { ESP } & \text { Environmental Simulation Program } \\ \text { GRE } & \text { Gas Release Event } \\ \text { HVAC } & \text { Heating, ventilating, and air conditioning } \\ \text { I\&C } & \text { Instrumentation and control } \\ \text { LANL } & \text { Los Alamos National Laboratory } \\ \text { LAW } & \text { Low-activity waste } \\ \text { M\&C } & \text { Monitor and control } \\ \text { NESHAP } & \text { National Emission Standards for Hazardous Air Pollutants } \\ \text { NPSH } & \text { Net Positive Suction Head } \\ \text { PNNL } & \text { - Pacific Northwest National Laboratory } \\ \text { RAM } & \text { Reliability, availability, maintainability } \\ \text { RCRA } & \text { Resource Conservation and Recovery Act of 1976 } \\ \text { TAP } & \text { Toxic Air Pollutants } \\ \text { TBD } & \text { To be determined } \\ \text { TWRS } & \text { Tank Waste Remediation System } \\ \text { TWRSO\&UP } & \text { Tank Waste Remediation System Operation and Utilization Plan } \\ \text { WAC } & \text { Washington Administrative Code } \\ \text { WFD } & \text { Waste Feed Delivery }\end{array}$


HNF-2238

Revision 0

EVALUATION OF TANK WASTE TRANSFERS AT 241-AW TANK FARM

\subsection{INTRODUCTION}

A number of waste transfers are needed to process and feed waste to the private contractors in support of Phase 1 Privatization. Other waste transfers are needed to support the 242-A Evaporator, saltwell pumping, and other ongoing Tank Waste Remediation System (TWRS) operations.

\subsection{PURPOSE OF EVALUATION}

The purpose of this evaluation is to determine if existing or planned equipment and systems are capable of supporting the Privatization Mission of the Tank Farms and continuing operations through the end of Phase 1B Privatization Mission. Projects W-211 and W-314 have been established and will support the privatization effort. Equipment and system upgrades provided by these projects (W-211 and W-314) will also support other ongoing operations in the tank farms. It is recognized that these projects do not support the entire transfer schedule represented in the Tank Waste Remediation System Operation and Utilization Plan (TWRSO\&UP) (Kirkbride et al. 1997). Additionally, transfers surrounding the 241-AW farm must be considered. This evaluation is provided as information, which will help to define transfer paths required to complete the Waste Feed Delivery (WFD) mission. This document is not focused on changing a particular project, but it is realized that new project work in the 241-AW Tank Farm is required.

\subsection{BACKGROUND INFORMATION}

The transfers identified in the TWRSO\&UP (Kirkbride et al. 1997) have been reviewed and specific transfers were extracted for evaluation. The transfers evaluated either begin or end in one of the six 241-AW tanks. All six of the 241-AW Tank Farm tanks are involved in at least one planned transfer. Approximately 180 separate transfers were identified. Transfer routes are dependent upon the timeframe (i.e., after fiscal year 2000, the new W-314 waste transfer system will be used along with existing pipelines).

From the 180 transfers, 32 different source and receiver tank combinations were identified and engineering judgement was used to postulate transfer routes. In postulating the transfer routes, the most direct $7.6 \mathrm{~cm}$ (3-in.) line was used (transfers from the 242A Evaporator to tank 241-AW-106 use $5.1 \mathrm{~cm}$ (2-in.) lines). Equipment and systems included in the 32 separate transfer routes were evaluated using the best available drawings and other data obtained from the pump and transfer cognizant engineers, and from project information. At 
this point in the evaluation missing pieces of the transfer routings such as jumpers in valve or pump pits were identified.

The 180 transfers were then grouped into three categories:

1. Feed Delivery. This category includes transfers of diluted supernate, and slurry from tank 241-AW-101 to tanks 241-AP-102/104 and supernate transfers from tank 241-AW-104 to tanks 241-AP-102/104.

2. Solids Cleanout from Tank 241-SY-102. This category includes only one transfer from tank 241-SY-102 to tank 241-AW-105. The transfer from tank 241-SY-102 to tank 241-AW-105 includes approximately 4 vol\% insoluble solids.

3. Other Transfers. This category includes the remaining approximately 175 transfers. Transfers in the "other" category are characterized as supernate transfers expected to contain less than 1 vol\% solids.

Each category of transfers involves a different set of requirements as compared to the other two. As an example, tank 241-AW-101 Contains saturated salt solutions, a crust layer on the top of the tank, and a layer of sludge on the bottom of the tank. Dilution studies have not been performed on the tank, but Environmental Simulation Program (ESP) ${ }^{1}$ modeling (included in Appendix D) indicates that most of the solids in the tank, both in the crust layer and in the sludge, are soluble. The requirements for waste retrieval from this tank will likely include: mixing the tank contents to degas; adding diluent (raw water) at the pump inlet to prevent solids from precipitating from saturated solution in the transfer line; ensuring that the pump is capable of transferring waste at a velocity of 1.8 to $2.7 \mathrm{~m} / \mathrm{s}(6$ to $9 \mathrm{ft} / \mathrm{s})$; and finally adding diluent (raw water) directly to the tank to ensure that as much as possible of the soluble solids are dissolved and removed from the tank. Requirements for the transfers from other tanks evaluated may not include mixing, dilution, or as high of a transfer rate.

A listing of the requirements for each of the three categories was developed using Performance Requirements for Phase 1 Waste Feed Delivery (HNF-1985) (Claghorn et al. 1998) for category 1 transfers and engineering judgement for category 2 and 3 transfers identified above.

Pressure drop and flow rate calculations were done for a number of transfer lengths using pump curves for two different pumps and estimated density and viscosity values. These calculations are then used to estimate pressure drops and flow rates for all 32 transfer routes. Tank temperatures were evaluated and precipitation due to temperature drop was estimated for the worst case tank. Based on Calculation No W314-P-006, (A.N. Palit March 12, 1997) a temperature drop of $6^{\circ} \mathrm{C}\left(10^{\circ} \mathrm{F}\right)$ in the transfer lines was used in the evaluation.

IESP is a trademark of OLI Systems, Inc. 
Also included in this evaluation was a review of open issues related to safety and environmental permitting of Tank Farm activities.

\subsection{DOCUMENT ORGANIZATION}

The main body of this document includes only a summary of the evaluation. Backup information and calculations are contained in the appendices. Section 2.0 contains process and equipment expectations and a summary of identified issues. Section 3.0 provides the conclusions and recommendations.

\subsubsection{Road Map to Appendices}

Appendix A contains a table listing the 32 transfer routes and the equipment and systems identified for each route. Existing equipment and planned equipment are identified as such and missing equipment is also identified. A sketch of the 241-AW Tank Farm lines including the connections to the 241-AP Valve Pit, 241-A-A Valve Pit, and to the 242-A Evaporator is also included in Appendix A. Missing jumper connections are shown on the sketch as heavy lines. The transfer schedule from the TWRSO\&UP (Kirkbride et al. 1997) is also included.

Appendix B contains all of the issues identified in this evaluation. Some of the issues are general in nature and others apply to a few or even only one transfer. An opportunity was also identified in this evaluation, and is included in the issues list. These issues are summarized in Section 2.3.

Appendix $\mathrm{C}$ contains a table of process and equipment requirements for each of the three identified categories of transfers. The basis of the requirements, and potential issues are also listed.

Appendix D contains the calculations of pressure drop and flow rates along each of the 32 transfer routes. The section also includes the temperature evaluation.

Appendix E contains the basis and requirements to assign work scope to resolve issues within the AW Tank Farm. 
HNF-2238

Revision 0

This page intentionally left blank. 
HNF-2238

Revision 0

\subsection{PROCESS AND EQUIPMENT EXPECTATIONS}

This section is a list of process and equipment expectations. This is a summary and does not list the bases for these expectations. The bases for these expectations are listed in Appendix C. As a summary, this list is not intended to give details. The details for each process or equipment expectation are listed in Appendix C.

\subsection{PROCESS EXPECTATIONS}

The process expectations are as follows:

- Transfer rates for Phase 1 waste feed delivery and for slurries will be maintained at 1.8 to $2.7 \mathrm{~m} / \mathrm{sec}(6$ to $9 \mathrm{ft} / \mathrm{sec})$.

- Temperatures and concentrations will be controlled and maintained during transfers such that no precipitates are formed.

- Supernates will contain no more than 5 vol\% suspended solids.

- Solutions transferred to the evaporator will be dilute and will meet evaporator requirements.

- Evaporator will not generate solutions from which solids will form under expected storage conditions.

\subsection{EQUIPMENT EXPECTATIONS}

The equipment expectations are as follows:

- Tanks from which supernates are to be transferred will have appropriate pumps and ancillary equipment such that solids in solution can be limited to required levels.

- Tanks from which slurries are to be transferred will be equipped with the capability to suspend the settled solids into a slurry, and will have pumps capable of transferring slurries.

- Tanks will be equipped with pumps which are capable of transferring waste at the rate required for the transfer.

- Where necessary, tanks will be equipped with the capability of diluting or heating the waste to ensure that no precipitates are formed during the transfer. 
HNF-2238

\section{Revision 0}

- Tanks will have liquid level measuring capability.

- System will be capable of monitoring tank levels.

- For as-low-as-reasonably-achievable (ALARA) radiation exposure reasons, system will be capable of making routing changes in valve pits without routine pit entry.

\subsection{ISSUES}

The following issues related to transfers originating or terminating in the 241-AW Tank Farm were identified during this evaluation. Complete descriptions and discussions of these issues are included in Appendix B.

\section{General}

- Current risk and RAM analysis efforts are not yet complete.

- Engineering studies that are aimed at optimizing the WFD system are not yet complete.

- The transfer schedule identified in the TWRSO\&UP (Kirkbride et al. 1997) reflects staging transfers that are not agreed to by TWRS operations. (Changes to the transfer schedule will occur.)

- The transfer schedule identified in the TWRSO\&UP (Kirkbride et al. 1997) reflects transfer rates that cannot be achieved by existing or planned equipment. (The TWRSO\&UP will be revised.)

- Dilution/dissolution studies have not been performed for tank 241-AW-101.

\section{Transfer Pumps}

- The existing pumps in the AW Farm are either incapable or only marginally capable of delivering feed at the required 1.8 to $2.7 \mathrm{~m} / \mathrm{s}(6$ to $9 \mathrm{ft} / \mathrm{s})$ velocity.

- A pump design is needed which allows periodic preventative maintenance (i.e. pump flushing, recirculation, etc.) to improve pump reliability.

- The existing transfer pump in tank 241-AW-104 has failed; work scope and funding to replace the pump have not been identified. 
HNF-2238

Revision 0

- The existing pumps in most of the tanks are stick type pumps that may not be appropriate for supernate transfers.

- The Design of the Existing Supernate Flex/Float Pumps Causes Pump to Lose Prime if Transfer is Interrupted.

\section{Mixer Pumps}

- Crust Softening/Removal Techniques are not Tested.

\section{Transfer Routings}

- The current jumper system in valve pits AW-A and AW-B consists of flex and rigid jumpers and is inadequate to support transfers identified in the TWRSO\&UP (Kirkbride et al. 1997).

- Current Jumper System in Pump Pit 241-AW-02A lacks the flexibility to support all of the transfers identified in the TWRSO\&UP (Kirkbride et al. 1997).

- Line 3-in. SN-219-M25 is not operable due to the existence of potentially leaky cleanout boxes in the line.

- Abandonment of 241-A-A valve pit requires that line LIQW-702 be connected directly to line SN-220.

\section{Regulatory Compliance}

- Compliance of the Transfer Lines with Washington Administrative Code (WAC) 173-303, Dangerous Waste Regulations.

- Concrete line encasements are not accepted by the Washington State Department of Ecology as appropriate.

- Ancillary Equipment Secondary Containment may need coatings or liners

- Written Integrity Assessment has not been completed

\section{Safety}

- System upgrades may be required to support Authorization Basis/Basis for Interim Operation (BIO) 
HNF-2238

Revision 0

- It will be difficult to isolate malfunctioning systems from systems which are functioning because the BIO does not accept valves as an appropriate means of isolating active transfer equipment from inactive systems.

\section{Instrumentation/Ancillary Equipment}

- Real time automatic liquid level detection is not available in all tanks.

- Equipment/instrument control strategies are not consistent throughout the farms.

- The current system is very complex.

- Integration requirements for new and existing instrumentation and controls have not been established.

\section{Ventilation}

- Electrical supply and control circuits to primary fans were identified in 1995 as being in poor condition.

- 241-AW primary ventilation stack will likely require upgrades to provide continuous air emissions monitoring.

- 241-AW primary ventilation system may require emission controls for Toxic Air Pollutants (TAPs).

- 241-AW-101 primary ventilation exhaust has no flow measurement capability.

\section{Electrical}

- A 1995 assessment found numerous deficiencies with MCC-241-AW.

- Retrieval systems added to tank 241-AW-101 will add a significant load to the 241-AW farm electrical system.

\section{Utilities}

- Transfer line flush water flow requirements have not been established.

- The 241-AW air compressors cycle frequently resulting in early failures 
HNF-2238

Revision 0

Waste Retrieval

- Process requirements have not been completed for retrieval of waste from 241-AW-101. 
HNF-2238

Revision 0

This page intentionally left blank. 
HNF-2238

Revision 0

\subsection{CONCLUSIONS AND RECOMMENDATIONS}

This evaluation has identified a number of questions and issues regarding the capability of the system to support the TWRSO\&UP (Kirkbride et al. 1997) identified transfers with existing or planned equipment. Some of the issues such as the failed transfer pump in tank 241-AW-104, and the lack of jumper connections in Valve Pit 241-AW-A/B would need to have already been addressed to support the identified transfers. (Two supernate transfers from AW-104 are scheduled to occur later this calendar year.)

Some of the issues such as the compliance of the existing lines with dangerous waste regulations are already being negotiated. The outcome of these negotiations is uncertain and furthermore the ramifications of negative outcomes are potentially extremely costly in terms of both budget and schedule.

Other issues such as the lack of jumpers in the AW-A and AW-B Valve Pits, and the potentially inappropriate pump types in the AW (and other farms as well) will require work scope and funding to make the necessary modifications.

A much more detailed evaluation of all the transfers should be undertaken as soon as possible. This evaluation has limited its scope to transfers originating or terminating in the 241-AW Tank Farm. The detailed evaluation should look at all transfers through the end of Phase 1 of the Waste Feed delivery mission. The evaluation should produce a complete list of performance requirements for all of the transfers. The evaluators should be given authority to make necessary changes or additions to work scope.

The opportunity of using tank 241-AW-101 as a backup in the case of a component failure in the primary transfer route from tank 241-AN-105 to the Phase 1 waste feed staging tanks should be implemented. This unique opportunity to ensure timely feed delivery and fee avoidance needs to be started now so that the W-211 Project schedule can be adjusted or accelerated as necessary before the 2001 delivery date. 
HNF-2238

Revision 0

This page intentionally left blank. 
HNF-2238

Revision 0

\subsection{REFERENCES}

Claghorn, R. D., I. G. Papp, and B. B. Peters (MACTEC), 1998, Performance Requirements for Phase 1 Waste Feed Delivery Components, HNF1985, Rev. 0, Numatec Hanford Corporation, Richland, Washington.

Golberg, R. L., 1996, DST and DCRT Tank Farm Electrical Distribution Systems Initial Assessment, WHC-SD-W314-ES-020, Rev. 0, Westinghouse Hanford Company, Richland, Washington.

Grams, W. H., W. L. Cowley, and K. H. Morris, 1997, Authorization Basis Assessment of Waste Feed Delivery, HNF-1948, Rev. 0, Duke Engineering and Services Hanford, Richland, Washington.

Kirkbride, R. A., G. K. Allen, P. J. Certa, A. F. Manuel, R. M. Orme, L. W. Shelton, E. J. Slaathaug, R. S. Wittman, and G. T. MacLean and D. L. Penwell (SESC), 1997, Tank Waste Remediation System Operation and Utilization Plan, HNF-SD-WM-SP-012, Rev. 0, Vol. I and II, Numatec Hanford Corporation, Richland, Washington.

Kriskovich, J., 1996, Initial Assessment Report HVAC Systems, WHC-SD-W314-ES-022, Rev. 0, Westinghouse Hanford Company, Richland, Washington.

LANL, 1992, Los Alamos National Laboratory Safety Assessment for the 241-SY-101 Mixer Pump, LA-UR-92-3196, Los Alamos National Laboratory, Los Alamos, New Mexico.

Merrick and Associates, and W. L. Willis, 1996, Hot Conditioning System Equipment Conceptual Design Report, WHC-SD-SNF-CDR-007, Rev. 0, Westinghouse Hanford Company, Richland, Washington.

Riesenweber, S. D. 1992, Tank Farms Pumping Equipment List, WHC-SD-WM-RPT-025, Rev. 1, Westinghouse Hanford Company, Richland, Washington.

WAC 173-303, "Dangerous Waste Regulations," Washington Administrative Code, as amended.

Willingham, W. E., Jr., 1997a, Technical Basis for Installation of the Double-Shell Tank Exhaust Flow Monitoring Systems, HNF-SD-WM-ER-629, Rev. 0, SGN Eurisys Services Corporation, Richland, WA.

Willingham, W. E., Jr., 1997b, Double-Shell Tank Primary Ventrilation Exhaust Flow Monitor System Design Description, HNF-SD-WM-SDD-074, Rev. 0, SGN Eurisys Services Corporation, Richland, WA. 
HNF-2238

Revision 0

This page intentionally left blank. 
HNF-2238

Revision 0

\section{APPENDIX A}

\section{TRANSFERS}


HNF-2238

Revision 0

This page intentionally left blank. 
HNF-2238

Revision 0

APPENDIX A

TRANSFERS

Appendix A contains a partial list of transfers which are included in the Tank Waste Remediation System Operation and Utilization Plan (TWRSO\&UP) (Kirkbride et al. 1997). The transfers listed here are scheduled to begin or end in the 241-AW Tank Farm. The first transfer listed here is scheduled to occur in calendar year 1998. The last transfer listed here is scheduled to occur in 2011. A route for each of the transfer paths was then postulated, and the existence of or plan for all necessary equipment in each transfer route was verified on best available drawings, project documents, and other available information.

Appendix A also includes a sketch of the necessary jumper connections in the AW-A and AW-B valve pits and the $241-\mathrm{AW}-102$ $02 \mathrm{~A}$ central pump pit.

Table A-1 is a listing of the transfers. Table A-2 is a listing of necessary equipment in each transfer route. Equipment is identified as either existing or planned. Table A-3 is a listing of the pipe material pressure and temperature limitation of the piping in the 241-AW Tank Farm. 
HNF-2238

Revision 0

This page intentionally left blank. 
HNF-2238

Revision 0

Table A-1. Transfers. (6 Sheets)

\begin{tabular}{|c|c|c|c|c|c|c|c|c|c|}
\hline $\begin{array}{l}\text { Boot } \\
\text { Strap }\end{array}$ & $\begin{array}{l}\text { HLW } \\
\text { Flag }\end{array}$ & $\begin{array}{l}\text { LAW } \\
\text { Flag }\end{array}$ & From & To & $\begin{array}{l}\text { Start } \\
\text { Date }\end{array}$ & $\begin{array}{l}\text { End } \\
\text { Date }\end{array}$ & $\begin{array}{l}\text { Liquid } \\
\text { (gal) }\end{array}$ & $\begin{array}{l}\text { Solid } \\
\text { (gal) }\end{array}$ & Comments \\
\hline & & & AW-106 & AW-101 & $3 / 1 / 03$ & $3 / 3 / 03$ & 550,400 & 26 & \\
\hline & & & AW-106 & AW-101 & $7 / 1 / 03$ & $7 / 1 / 03$ & 34,750 & 2 & \\
\hline & & & AW-106 & AW-101 & $1 / 1 / 04$ & $1 / 1 / 04$ & 746 & 0 & \\
\hline & & & AW-106 & AW-101 & $7 / 1 / 04$ & $7 / 1 / 04$ & 11,151 & 1 & \\
\hline & & & AW-106 & AW-101 & $1 / 1 / 05$ & $1 / 1 / 05$ & 95,306 & 4 & \\
\hline & & & AW-106 & AW-101 & $7 / 1 / 05$ & $7 / 1 / 05$ & 121,700 & 6 & \\
\hline & & & AW-106 & AW-101 & $1 / 1 / 06$ & $1 / 1 / 06$ & 51,422 & 2 & \\
\hline & & & AW-106 & AW-101 & $7 / 1 / 06$ & $7 / 1 / 06$ & 22,041 & 1 & \\
\hline & & & AW-106 & AW-101 & $1 / 1 / 07$ & $1 / 1 / 07$ & 74,822 & 3 & \\
\hline & & & AW-106 & AW-101 & $7 / 1 / 07$ & $7 / 1 / 07$ & 33,387 & 2 & \\
\hline & & & AW-106 & AW-101 & $1 / 1 / 08$ & $1 / 1 / 08$ & 1,111 & 0 & \\
\hline & & & AW-106 & AW-101 & $7 / 1 / 08$ & $7 / 1 / 08$ & 12,300 & 1 & \\
\hline & & & AW-106 & AW-101 & $1 / 1 / 09$ & $1 / 1 / 09$ & 867 & 0 & \\
\hline & & & AW-106 & AW-101 & $7 / 1 / 09$ & $7 / 1 / 09$ & 10,622 & 0 & \\
\hline & & & AW-106 & AW-101 & $1 / 1 / 10$ & $1 / 1 / 10$ & 560 & 0 & \\
\hline & & & AW-106 & AW-101 & $7 / 1 / 10$ & $7 / 1 / 10$ & 16,078 & 1 & \\
\hline & & & AW-106 & AW-101 & $1 / 1 / 11$ & $1 / 1 / 11$ & 1,173 & 0 & \\
\hline & & & AW-106 & AW-101 & $7 / 1 / 11$ & $7 / 1 / 11$ & 0 & 0 & \\
\hline & & & & & & & & & \\
\hline & & $\mathrm{Y}$ & AW-101 & AP-102 & $1 / 9 / 03$ & $1 / 13 / 03$ & 537,200 & 13,013 & $\begin{array}{l}\text { Retrieve/Stage LAW } \\
\text { Batch 3, Contractor 1, } \\
\text { Envelope A }\end{array}$ \\
\hline & & $Y$ & AW-101 & AP-102 & $1 / 9 / 03$ & $1 / 13 / 03$ & 845,400 & 13,013 & As-received Ghost \\
\hline & & $\mathrm{Y}$ & AW-101 & AP-104 & $1 / 14 / 03$ & $1 / 18 / 03$ & 537,200 & 13,013 & $\begin{array}{l}\text { Retrieve/Stage LAW } \\
\text { Batch 3, Contractor 2, } \\
\text { Envelope A }\end{array}$ \\
\hline & & $Y$ & AW-101 & AP-104 & $1 / 14 / 03$ & $1 / 18 / 03$ & 845,400 & 13,013 & As-received Ghost \\
\hline & & & & & & & & & \\
\hline & & & AP-104 & AW-102 & $3 / 1 / 98$ & $3 / 6 / 98$ & 995,300 & 34 & \\
\hline & & & AP-107 & AW-102 & $4 / 25 / 98$ & $5 / 7 / 98$ & $1,104,000$ & 40 & \\
\hline & & & EVAPF & AW-102 & $10 / 13 / 98$ & $10 / 14 / 98$ & 195,000 & 0 & \\
\hline & & & AW-106 & AW-102 & $11 / 6 / 98$ & $11 / 6 / 98$ & 150,000 & 6 & \\
\hline & & & AP-104 & AW-102 & $10 / 3 / 98$ & $11 / 10 / 98$ & $1,110,000$ & 42 & \\
\hline & & & AP-107 & AW-102 & $2 / 15 / 99$ & $3 / 9 / 99$ & $1,095,000$ & 40 & \\
\hline & & & AP-103 & AW-102 & $7 / 1 / 99$ & $7 / 8 / 99$ & $1,017,000$ & 44 & \\
\hline \multirow[t]{4}{*}{$\mathrm{Y}$} & & & EVAPF & AW-102 & $9 / 7 / 99$ & $9 / 7 / 99$ & 70,000 & 0 & \\
\hline & & $\mathrm{Y}$ & AP-104 & AW-102 & $9 / 10 / 99$ & $9 / 29 / 99$ & $1,104,000$ & 41 & \\
\hline & & & AW-106 & AW-102 & $10 / 6 / 99$ & $10 / 7 / 99$ & 300,000 & 13 & \\
\hline & & & AP-107 & AW-102 & $11 / 1 / 99$ & $11 / 8 / 99$ & $1,110,000$ & 43 & \\
\hline $\mathrm{Y}$ & & & AP-104 & AW-102 & $7 / 2 / 00$ & $7 / 8 / 00$ & $1,110,000$ & 43 & \\
\hline
\end{tabular}


HNF-2238

Revision 0

Table A-1. Transfers. (6 Sheets)

\begin{tabular}{|c|c|c|c|c|c|c|c|c|c|}
\hline $\begin{array}{l}\text { Boot } \\
\text { Strap }\end{array}$ & $\begin{array}{c}\text { HLW } \\
\text { Flag }\end{array}$ & $\begin{array}{l}\text { LAW } \\
\text { Flag }\end{array}$ & From & To & $\begin{array}{l}\text { Start } \\
\text { Date }\end{array}$ & $\begin{array}{l}\text { End } \\
\text { Date }\end{array}$ & $\begin{array}{l}\text { Liquid } \\
\text { (gal) }\end{array}$ & $\begin{array}{l}\text { Solid } \\
\text { (gal) }\end{array}$ & Comments \\
\hline $\mathrm{Y}$ & & $\mathrm{Y}$ & AP-106 & AW-102 & $10 / 2 / 00$ & $10 / 4 / 00$ & 474,000 & 16 & \\
\hline$Y$ & & $Y$ & AP-106 & AW-102 & $10 / 5 / 00$ & $10 / 5 / 00$ & 71,998 & 2 & \\
\hline \multirow[t]{2}{*}{$\mathrm{Y}$} & & $Y$ & AP-106 & AW-102 & $10 / 6 / 00$ & $10 / 6 / 00$ & 130,000 & 4 & \\
\hline & & $\mathrm{Y}$ & AP-106 & AW-102 & $10 / 7 / 00$ & $10 / 7 / 00$ & 60,191 & 2 & \\
\hline \multirow[t]{34}{*}{$Y$} & & & AW-105 & AW-102 & $10 / 6 / 00$ & $10 / 8 / 00$ & 414,000 & 14 & \\
\hline & & $\mathrm{Y}$ & AP-106 & AW-102 & $10 / 8 / 00$ & $10 / 8 / 00$ & 0 & 0 & \\
\hline & & & AW-105 & AW-102 & $10 / 9 / 00$ & $10 / 10 / 00$ & 213,100 & 7 & \\
\hline & & & AP-106 & AW-102 & $2 / 7 / 01$ & $2 / 7 / 01$ & 0 & 0 & \\
\hline & & & SY-102 & AW-102 & $4 / 4 / 01$ & $4 / 5 / 01$ & 384,000 & 16 & \\
\hline & & & SY-102 & AW-102 & $4 / 6 / 01$ & $4 / 6 / 01$ & 10,000 & 0 & \\
\hline & & & AN-105 & AW-102 & $6 / 10 / 01$ & $6 / 12 / 01$ & 520,100 & 21 & \\
\hline & & & WATER & AW-102 & $10 / 19 / 01$ & $10 / 19 / 01$ & 35,000 & 0 & \\
\hline & & & EVAPF & AW-102 & $10 / 19 / 01$ & $10 / 20 / 01$ & 225,000 & 0 & \\
\hline & & & AN-105 & AW-102 & $12 / 10 / 01$ & $12 / 10 / 01$ & 35,011 & 1 & \\
\hline & & & SY-102 & AW-102 & $4 / 4 / 02$ & $4 / 4 / 02$ & 29,999 & 1 & \\
\hline & & & SY-102 & AW-102 & $4 / 4 / 02$ & $4 / 4 / 02$ & 4,000 & 0 & \\
\hline & & & AN-105 & AW-102 & $6 / 10 / 02$ & $6 / 11 / 02$ & 281,100 & 10 & \\
\hline & & & EVAPF & AW-102 & $9 / 20 / 02$ & $9 / 20 / 02$ & 50,000 & 0 & \\
\hline & & & AN-105 & AW-102 & $12 / 10 / 02$ & $12 / 13 / 02$ & 681,500 & 23 & \\
\hline & & & SY-102 & AW-102 & $4 / 4 / 03$ & $4 / 4 / 03$ & 29,999 & 1 & \\
\hline & & & SY-102 & AW-102 & $4 / 4 / 03$ & $4 / 4 / 03$ & 4,000 & 0 & \\
\hline & & & AN-105 & AW-102 & $6 / 10 / 03$ & $6 / 10 / 03$ & 0 & 0 & \\
\hline & & & EVAPF & AW-102 & $9 / 20 / 03$ & $9 / 20 / 03$ & 35,000 & 0 & \\
\hline & & & AN-105 & AW-102 & $12 / 10 / 03$ & $12 / 11 / 03$ & 268,900 & 9 & \\
\hline & & & SY-102 & AW-102 & $4 / 4 / 04$ & $4 / 4 / 04$ & 29,999 & 1 & \\
\hline & & & SY-102 & AW-102 & $4 / 4 / 04$ & $4 / 4 / 04$ & 3,000 & 0 & \\
\hline & & & AN-105 & AW-102 & $5 / 12 / 04$ & $5 / 12 / 04$ & 0 & 0 & \\
\hline & & & AN-105 & AW-102 & $6 / 10 / 04$ & $6 / 11 / 04$ & 231,200 & 9 & \\
\hline & & & EVAPF & AW-102 & $9 / 20 / 04$ & $9 / 20 / 04$ & 85,000 & 0 & \\
\hline & & & AN-105 & AW-102 & $12 / 10 / 04$ & $12 / 11 / 04$ & 258,900 & 9 & \\
\hline & & & AN-105 & AW-102 & $5 / 21 / 05$ & $5 / 25 / 05$ & 772,500 & 13,977 & \\
\hline & & & AN-105 & AW-102 & $6 / 10 / 05$ & $6 / 12 / 05$ & 390,600 & 14 & \\
\hline & & & EVAPF & AW-102 & $9 / 20 / 05$ & $9 / 20 / 05$ & 35,000 & 0 & \\
\hline & & & AN-105 & AW-102 & $12 / 10 / 05$ & $12 / 12 / 05$ & 581,100 & 20 & \\
\hline & & & AN-105 & AW-102 & $6 / 10 / 06$ & $6 / 14 / 06$ & 872,900 & 30 & \\
\hline & & & AN-105 & AW-102 & $12 / 10 / 06$ & $12 / 12 / 06$ & 431,900 & 15 & \\
\hline & & & $S Y-102$ & AW-102 & $4 / 4 / 07$ & $4 / 4 / 07$ & 29,195 & 1 & \\
\hline & & & AN-105 & AW-102 & $6 / 10 / 07$ & $6 / 10 / 07$ & 0 & 0 & \\
\hline
\end{tabular}


HNF-2238

Revision 0

Table A-1. Transfers. (6 Sheets)

\begin{tabular}{|c|c|c|c|c|c|c|c|c|c|}
\hline $\begin{array}{l}\text { Boot } \\
\text { Strap }\end{array}$ & $\begin{array}{l}\text { HLW } \\
\text { Flag }\end{array}$ & $\begin{array}{l}\text { LAW } \\
\text { Flag }\end{array}$ & From & To & $\begin{array}{l}\text { Start } \\
\text { Date }\end{array}$ & $\begin{array}{l}\text { End } \\
\text { Date }\end{array}$ & $\begin{array}{l}\text { Liquid } \\
\text { (gal) }\end{array}$ & $\begin{array}{l}\text { Solid } \\
\text { (gal) }\end{array}$ & Comments \\
\hline & & & EVAPF & AW-102 & $9 / 20 / 07$ & 9/20/07 & 35,000 & 0 & \\
\hline & & & AN-105 & AW-102 & $12 / 10 / 07$ & $12 / 11 / 07$ & 259,900 & 9 & \\
\hline & & & SY-102 & AW-102 & $4 / 4 / 08$ & $4 / 4 / 08$ & 29,999 & 1 & \\
\hline & & & AN-105 & AW-102 & $6 / 10 / 08$ & $6 / 10 / 08$ & 0 & 0 & \\
\hline & & & EVAPF & AW-102 & $9 / 20 / 08$ & 9/20/08 & 85,000 & 0 & \\
\hline & & & AN-105 & AW-102 & $12 / 10 / 08$ & $12 / 11 / 08$ & 259,900 & 9 & \\
\hline & & & SY-102 & AW-102 & $4 / 4 / 09$ & $4 / 4 / 09$ & 29,999 & 1 & \\
\hline & & & AN-105 & AW-102 & $6 / 10 / 09$ & $6 / 10 / 09$ & 0 & 0 & \\
\hline & & & EVAPF & AW-102 & $9 / 20 / 09$ & $9 / 20 / 09$ & 35,000 & 0 & \\
\hline & & & AN-105 & AW-102 & $12 / 10 / 09$ & $12 / 11 / 09$ & 270,900 & 9 & \\
\hline & & & SY-102 & AW-102 & $4 / 4 / 10$ & $4 / 4 / 10$ & 29,999 & 1 & \\
\hline & & & AN-105 & AW-102 & $6 / 10 / 10$ & $6 / 10 / 10$ & 0 & 0 & \\
\hline & & & EVAPF & AW-102 & $9 / 20 / 10$ & $9 / 20 / 10$ & 85,000 & 0 & \\
\hline & & & EVAPF & AW-102 & $10 / 19 / 10$ & $10 / 19 / 10$ & 35,000 & 0 & \\
\hline & & & AN-105 & AW-102 & $12 / 10 / 10$ & $12 / 11 / 10$ & 285,900 & 10 & \\
\hline & & & SY -102 & AW-102 & $4 / 4 / 11$ & $4 / 4 / 11$ & 29,360 & 640 & \\
\hline & & & AN-105 & AW-102 & $6 / 10 / 11$ & $6 / 10 / 11$ & 50,020 & 2 & \\
\hline & & & & & & & & & \\
\hline & & & AW-102 & 242-A & $4 / 5 / 98$ & $4 / 9 / 98$ & 995,300 & 35 & \\
\hline & & & AW-102 & 242-A & $5 / 5 / 98$ & $5 / 10 / 98$ & $1,104,000$ & 39 & \\
\hline & & & AW-102 & AW-106 & $10 / 28 / 98$ & $10 / 28 / 98$ & 150,000 & 6 & \\
\hline & & & AW-102 & $242-A$ & $11 / 5 / 98$ & $11 / 10 / 98$ & $1,195,000$ & 46 & \\
\hline & & & AW-102 & $242-\mathrm{A}$ & $3 / 5 / 99$ & $3 / 10 / 99$ & $1,130,000$ & 36 & \\
\hline & & & AW-102 & $242-A$ & $7 / 5 / 99$ & $7 / 9 / 99$ & 979,200 & 36 & \\
\hline & & & AW-102 & AW-106 & 9/22/99 & $9 / 23 / 99$ & 300,000 & 12 & \\
\hline & & & AW-102 & $242-A$ & $10 / 5 / 99$ & $10 / 10 / 99$ & $1,139,000$ & 45 & \\
\hline & & & AW-102 & $242-\mathrm{A}$ & $11 / 5 / 99$ & $11 / 10 / 99$ & $1,110,000$ & 34 & \\
\hline & & & AW-102 & $242-\mathrm{A}$ & $7 / 6 / 00$ & $7 / 11 / 00$ & $1,145,000$ & 36 & \\
\hline & & & AW-102 & $242-\mathrm{A}$ & $10 / 5 / 00$ & $10 / 12 / 00$ & $1,476,000$ & 52 & \\
\hline & & & AW-102 & $242-\mathrm{A}$ & $6 / 1 / 01$ & $6 / 3 / 01$ & 394,000 & 16 & \\
\hline & & & AW-102 & $242-\mathrm{A}$ & $12 / 1 / 01$ & $12 / 4 / 01$ & 780,100 & 30 & \\
\hline & & & AW-102 & $242-\mathrm{A}$ & $6 / 1 / 02$ & $6 / 1 / 02$ & 69,010 & 3 & \\
\hline & & & AW-102 & $242-\mathrm{A}$ & $12 / 1 / 02$ & $12 / 3 / 02$ & 331,100 & 11 & \\
\hline & & & AW-102 & $242-\mathrm{A}$ & $6 / 1 / 03$ & $6 / 4 / 03$ & 715,500 & 24 & \\
\hline & & & AW-102 & $242-\mathrm{A}$ & $12 / 1 / 03$ & $12 / 1 / 03$ & 35,011 & 1 & \\
\hline & & & $\mathrm{AW}-102$ & $242-\mathrm{A}$ & $6 / 1 / 04$ & $6 / 2 / 04$ & 301,900 & 10 & \\
\hline & & & AW-102 & $242-\mathrm{A}$ & $12 / 1 / 04$ & $12 / 2 / 04$ & 316,200 & 12 & \\
\hline & & & AW-102 & $242-\mathrm{A}$ & $6 / 1 / 05$ & $6 / 5 / 05$ & 872,800 & 31 & \\
\hline
\end{tabular}


HNF-2238

Revision 0

Table A-1. Transfers. (6 Sheets)

\begin{tabular}{|c|c|c|c|c|c|c|c|c|c|}
\hline $\begin{array}{l}\text { Boot } \\
\text { Strap }\end{array}$ & $\begin{array}{c}\text { HLW } \\
\text { Flag }\end{array}$ & $\begin{array}{l}\text { LAW } \\
\text { Flag }\end{array}$ & From & To & $\begin{array}{l}\text { Start } \\
\text { Date }\end{array}$ & $\begin{array}{l}\text { End } \\
\text { Date }\end{array}$ & $\begin{array}{l}\text { Liquid } \\
\text { (gal) }\end{array}$ & $\begin{array}{l}\text { Solid } \\
\text { (gal) }\end{array}$ & Comments \\
\hline & & & AW-102 & 242-A & $11 / 22 / 05$ & $11 / 25 / 05$ & 425,600 & 15 & \\
\hline & & & AW-102 & 242-A & $12 / 1 / 05$ & $12 / 1 / 05$ & 0 & 0 & \\
\hline & & & $\mathrm{AW}-102$ & $242-\mathrm{A}$ & $6 / 1 / 06$ & $6 / 4 / 06$ & 581,100 & 20 & \\
\hline & & & $\mathrm{AW}-102$ & $242-\mathrm{A}$ & $12 / 1 / 06$ & $12 / 5 / 06$ & 872,900 & 30 & \\
\hline & & & AW-102 & $242-\mathrm{A}$ & $6 / 1 / 07$ & $6 / 3 / 07$ & 461,100 & 16 & \\
\hline & & & AW-102 & $242-\mathrm{A}$ & $12 / 1 / 07$ & $12 / 1 / 07$ & 35,013 & 1 & \\
\hline & & & $\mathrm{AW}-102$ & 242-A & $6 / 1 / 08$ & $6 / 2 / 08$ & 289,900 & 10 & \\
\hline & & & AW-102 & $242-\mathrm{A}$ & $12 / 1 / 08$ & $12 / 1 / 08$ & 85,032 & 3 & \\
\hline & & & AW-102 & 242-A & $6 / 1 / 09$ & $6 / 2 / 09$ & 289,900 & 10 & \\
\hline & & & AW-102 & $242-A$ & $12 / 1 / 09$ & $12 / 1 / 09$ & 35,013 & 1 & \\
\hline & & & $\mathrm{AW}-102$ & $242-\mathrm{A}$ & $6 / 1 / 10$ & $6 / 2 / 10$ & 300,900 & 10 & \\
\hline & & & AW-102 & $242-\mathrm{A}$ & $12 / 1 / 10$ & $12 / 2 / 10$ & 120,000 & 4 & \\
\hline & & & AP-101 & AW-103 & $8 / 6 / 98$ & $8 / 9 / 98$ & 625,000 & 27 & \\
\hline & & & AN-103 & AW-103 & $1 / 18 / 05$ & $1 / 18 / 05$ & 3,897 & 103 & \\
\hline & & & & & . & & & & \\
\hline & & & AP-101 & AW-104 & $10 / 21 / 98$ & $10 / 23 / 98$ & 460,000 & 20 & \\
\hline & & & AW-106 & AW-104 & $4 / 5 / 99$ & $4 / 5 / 99$ & 136,800 & 6 & \\
\hline & & & AW-106 & AW-104 & $10 / 12 / 99$ & $10 / 12 / 99$ & 111,000 & 5 & \\
\hline & & & AN-103 & AW-104 & $1 / 17 / 05$ & $1 / 18 / 05$ & 17,750 & 468 & \\
\hline & & & & & & & & & \\
\hline & & & AW-104 & AP-107 & $10 / 10 / 98$ & $10 / 12 / 98$ & 540,000 & 18 & \\
\hline & & & AW-104 & AP-104 & $10 / 14 / 98$ & $10 / 14 / 98$ & 164,000 & 6 & \\
\hline & & & 1NS96 & AW-105 & $9 / 18 / 00$ & $9 / 18 / 00$ & 15,002 & 4,998 & \\
\hline & & & WATER & AW-105 & $9 / 18 / 00$ & $9 / 18 / 00$ & 174,000 & 0 & \\
\hline & & & 1FL96 & AW-105 & $9 / 18 / 00$ & $9 / 19 / 00$ & 53,000 & 0 & \\
\hline & & & 1KL96 & AW-105 & $9 / 18 / 00$ & $9 / 19 / 00$ & 332,000 & 0 & \\
\hline & $\mathrm{Y}$ & & AZ-101 & AW-105 & $10 / 10 / 00$ & $10 / 10 / 00$ & 142,500 & 0 & First Wash Decant $1 \mathrm{AZ}$ \\
\hline & $\bar{Y}$ & & AZ-101 & AW-105 & $11 / 30 / 00$ & $12 / 1 / 00$ & 140,200 & 0 & $\begin{array}{l}\text { Second Wash Decant } \\
1 \mathrm{AZ}\end{array}$ \\
\hline & $\mathrm{Y}$ & & AZ-101 & $A W-105$ & $1 / 21 / 01$ & $1 / 21 / 01$ & 142,900 & 0 & $\begin{array}{l}\text { Third Wash Decant } \\
1 \mathrm{AZ}\end{array}$ \\
\hline & & & WATER & AW-105 & $9 / 19 / 04$ & $9 / 20 / 04$ & 88,000 & 0 & \\
\hline & & & 1FL96 & AW-105 & 9/19/04 & $9 / 20 / 04$ & 200,000 & 0 & \\
\hline & & $\mathrm{Y}$ & SY-102 & AW-105 & $1 / 1 / 06$ & $1 / 3 / 06$ & 580,400 & 22,129 & Clean out Solids in 2SY \\
\hline & & & AN-103 & AW-105 & $4 / 2 / 06$ & $4 / 4 / 06$ & 458,900 & 12,056 & \\
\hline & & & AW-105 & AW-102 & $10 / 6 / 00$ & $10 / 8 / 00$ & 414,000 & 14 & \\
\hline
\end{tabular}


HNF-2238

Revision 0

Table A-1. Transfers. (6 Sheets)

\begin{tabular}{|c|c|c|c|c|c|c|c|c|c|}
\hline $\begin{array}{l}\text { Boot } \\
\text { Strap }\end{array}$ & $\begin{array}{c}\text { HLW } \\
\text { Flag }\end{array}$ & $\begin{array}{c}\text { LAW } \\
\text { Flag }\end{array}$ & From & To & $\begin{array}{l}\text { Start } \\
\text { Date }\end{array}$ & $\begin{array}{l}\text { End } \\
\text { Date }\end{array}$ & $\begin{array}{l}\text { Liquid } \\
\text { (gal) }\end{array}$ & $\begin{array}{l}\text { Solid } \\
\text { (gal) }\end{array}$ & Comments \\
\hline & & & AW-105 & $\mathrm{AW}-102$ & $10 / 9 / 00$ & $10 / 10 / 00$ & 213,100 & 7 & \\
\hline & & & AW- 105 & AN-105 & $12 / 15 / 05$ & $12 / 18 / 05$ & 713,900 & 25 & \\
\hline & & & AW-105 & AN-105 & $3 / 1 / 06$ & $3 / 2 / 06$ & 321,000 & 11 & \\
\hline & & & & & & & & & \\
\hline & & & $242-\dot{A}$ & AW-106 & $4 / 5 / 98$ & $4 / 9 / 98$ & 168,500 & 35 & \\
\hline & & & $242-\mathrm{A}$ & AW-106 & $5 / 5 / 98$ & $5 / 10 / 98$ & 441,000 & 39 & \\
\hline & & & AW-102 & AW-106 & $10 / 28 / 98$ & $10 / 28 / 98$ & 150,000 & 6 & \\
\hline & & & $242-A$ & AW-106 & $11 / 5 / 98$ & $11 / 10 / 98$ & 585,000 & 46 & \\
\hline & & & $242-\mathrm{A}$ & AW-106 & $3 / 5 / 99$ & $3 / 10 / 99$ & 360,900 & 36 & \\
\hline & & & 242-A & AW-106 & $7 / 5 / 99$ & $7 / 9 / 99$ & 891,000 & 36 & \\
\hline & & & AW-102 & AW-106 & $9 / 22 / 99$ & $9 / 23 / 99$ & 300,000 & 12 & \\
\hline & & & $242-\mathrm{A}$ & AW-106 & $10 / 5 / 99$ & $10 / 10 / 99$ & 432,200 & 45 & \\
\hline & & & $242-A$ & AW-106 & $11 / 5 / 99$ & $11 / 10 / 99$ & 732,200 & 34 & \\
\hline & & & $242-\mathrm{A}$ & $A W-106$ & $7 / 6 / 00$ & $7 / 11 / 00$ & 778,300 & 36 & \\
\hline & & & $242-\mathrm{A}$ & AW-106 & $10 / 5 / 00$ & $10 / 12 / 00$ & 87,361 & 52 & \\
\hline & & & $242-\mathrm{A}$ & AW-106 & $6 / 1 / 01$ & $6 / 3 / 01$ & 211,900 & 16 & \\
\hline & & & $242-\mathrm{A}$ & AW-106 & $12 / 1 / 01$ & $12 / 4 / 01$ & 282,600 & 30 & \\
\hline & & & $242-\mathrm{A}$ & AW-106 & $6 / 1 / 02$ & $6 / 1 / 02$ & 21,399 & 3 & \\
\hline & & & $242-\mathrm{A}$ & AW-106 & $12 / 1 / 02$ & $12 / 3 / 02$ & 34,737 & 11 & \\
\hline & & & $242-A$ & AW-106 & $6 / 1 / 03$ & $6 / 4 / 03$ & 34,944 & 24 & \\
\hline & & & $242-A$ & AW-106 & $12 / 1 / 03$ & $12 / 1 / 03$ & 756 & 1 & \\
\hline & & & $242-\mathrm{A}$ & AW-106 & $6 / 1 / 04$ & $6 / 2 / 04$ & 11,234 & 10 & \\
\hline & & & $242-\mathrm{A}$ & AW-106 & $12 / 1 / 04$ & $12 / 2 / 04$ & 95,369 & 12 & \\
\hline & & & $242-\mathrm{A}$ & AW-106 & $6 / 1 / 05$ & $6 / 5 / 05$ & 121,900 & 31. & \\
\hline & & & $242-\mathrm{A}$ & AW-106 & $11 / 22 / 05$ & $11 / 25 / 05$ & 51,529 & 15 & \\
\hline & & & $242-\mathrm{A}$ & AW-106 & $12 / 1 / 05$ & $12 / 1 / 05$ & 0 & 0 & \\
\hline & & & 242-A & AW-106 & $6 / 1 / 06$ & $6 / 4 / 06$ & 22,200 & 20 & \\
\hline & & & $242-\mathrm{A}$ & AW-106 & $12 / 1 / 06$ & $12 / 5 / 06$ & 75,050 & 30 & \\
\hline & & & $242-\mathrm{A}$ & AW-106 & $6 / 1 / 07$ & $6 / 3 / 07$ & 33,509 & 16 & \\
\hline & & & $242-\mathrm{A}$ & AW-106 & $12 / 1 / 07$ & $12 / 1 / 07$ & 1,120 & 1 & \\
\hline & & & $242-A$ & AW-106 & $6 / 1 / 08$ & $6 / 2 / 08$ & 12,379 & 10 & \\
\hline & & & $242-A$ & AW-106 & $12 / 1 / 08$ & $12 / 1 / 08$ & 891 & 3 & \\
\hline & & & $242-\mathrm{A}$ & AW-106 & $6 / 1 / 09$ & $6 / 2 / 09$ & 10,702 & 10 & \\
\hline & & & $242-\mathrm{A}$ & AW-106 & $12 / 1 / 09$ & $12 / 1 / 09$ & 570 & 1 & \\
\hline & & & $242-\mathrm{A}$ & AW-106 & $6 / 1 / 10$ & $6 / 2 / 10$ & 16,159 & 10 & \\
\hline & & & $242-\mathrm{A}$ & AW-106 & $12 / 1 / 10$ & $12 / 2 / 10$ & 1,207 & 4 & \\
\hline & & & AW-106 & AP-105 & 6/3/98 & $6 / 4 / 98$ & 341,000 & 16 & \\
\hline
\end{tabular}


HNF-2238

Revision 0

Table A-1. Transfers. (6 Sheets)

\begin{tabular}{|c|c|c|c|c|c|c|c|c|c|}
\hline $\begin{array}{l}\text { Boot } \\
\text { Strap }\end{array}$ & $\begin{array}{c}\text { HLW } \\
\text { Flag }\end{array}$ & $\begin{array}{l}\text { LAW } \\
\text { Flag }\end{array}$ & From & To & $\begin{array}{l}\text { Start } \\
\text { Date }\end{array}$ & $\begin{array}{l}\text { End } \\
\text { Date }\end{array}$ & $\begin{array}{l}\text { Liquid } \\
\text { (gal) }\end{array}$ & $\begin{array}{l}\text { Solid } \\
\text { (gal) }\end{array}$ & Comments \\
\hline & & & AW-106 & AP-101 & $10 / 27 / 98$ & $10 / 28 / 98$ & 289,000 & 13 & \\
\hline & & & AW-106 & AW-102 & $11 / 6 / 98$ & $11 / 6 / 98$ & 150,000 & 6 & \\
\hline & & & AW-106 & AP-101 & $12 / 1 / 98$ & $12 / 3 / 98$ & 563,900 & 25 & \\
\hline & & & AW-106 & AP-101 & $4 / 3 / 99$ & $4 / 4 / 99$ & 224,000 & 10 & \\
\hline & & & AW-106 & AW-104 & $4 / 5 / 99$ & $4 / 5 / 99$ & 136,800 & 6. & \\
\hline & & & AW-106 & AN-106 & $7 / 20 / 99$ & $7 / 23 / 99$ & 748,000 & 33 & \\
\hline & & & AW-106 & AP-108 & $7 / 25 / 99$ & $7 / 25 / 99$ & 143,000 & 6 & \\
\hline & & & AW-106 & AW-102 & $10 / 6 / 99$ & $10 / 7 / 99$ & 300,000 & 13 & \\
\hline & & & AW-106 & AW-104 & $10 / 12 / 99$ & $10 / 12 / 99$ & 111,000 & 5 & \\
\hline & & & AW-106 & AN-101 & $10 / 13 / 99$ & $10 / 14 / 99$ & 321,000 & 16 & \\
\hline & & & AW-106 & AN-101 & $12 / 12 / 99$ & $12 / 15 / 99$ & 732,200 & 33 & \\
\hline & & & AW-106 & AP-107 & $9 / 14 / 00$ & $9 / 18 / 00$ & 778,200 & 34 & \\
\hline & & & AW-106 & AP-107 & $11 / 3 / 00$ & $11 / 3 / 00$ & 86,999 & 5 & \\
\hline
\end{tabular}

HLW $=$ High-level waste

LAW $=$ Low-activity waste. 


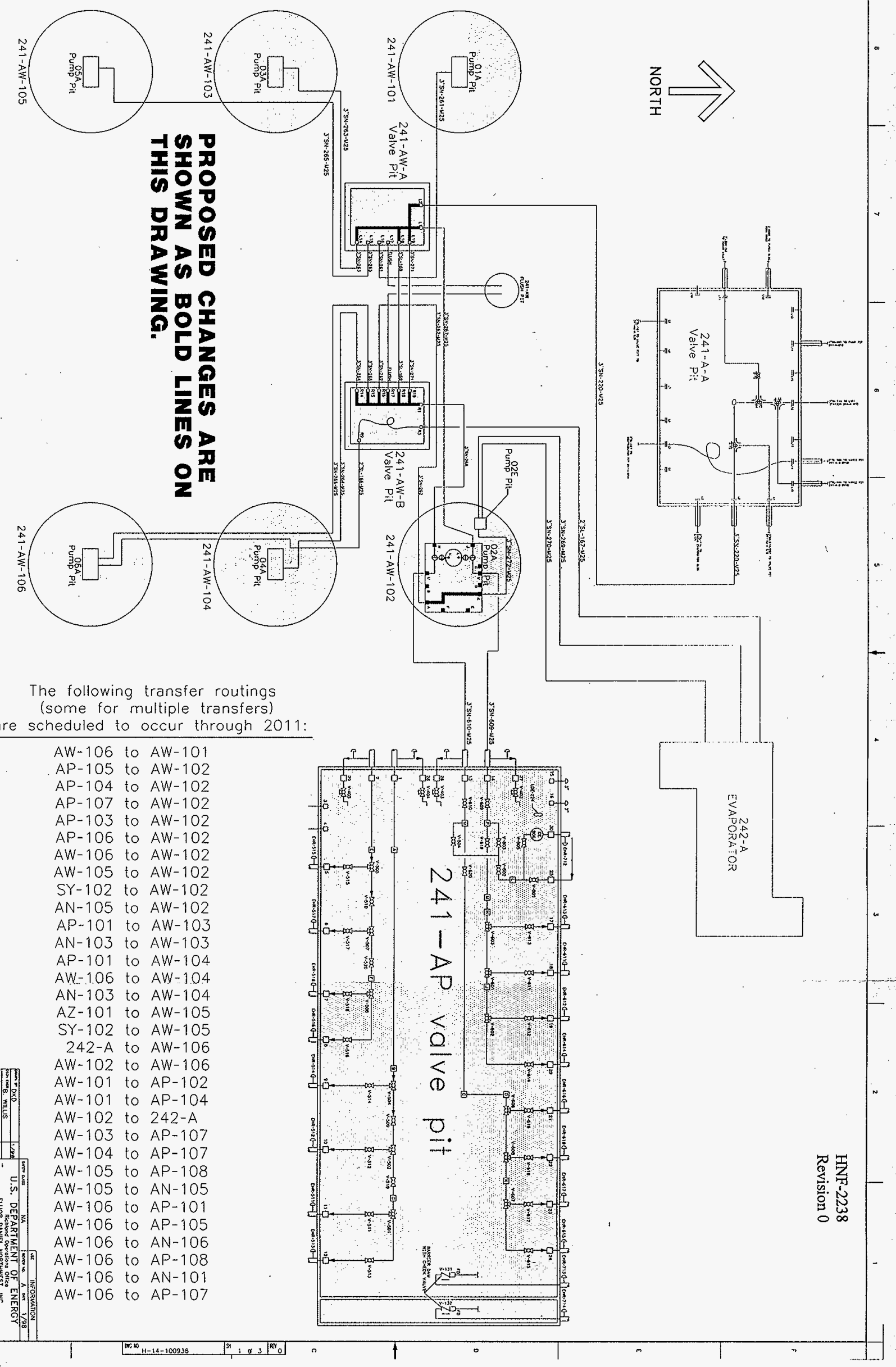


Table A-2. Equipment Availability Matrix.

\begin{tabular}{|c|c|c|c|c|c|c|c|}
\hline \multirow{2}{*}{ Transfer } & \multirow{2}{*}{ Transfer route } & \multirow{2}{*}{ Equipment needs } & \multicolumn{2}{|l|}{ Equipment installed } & \multicolumn{2}{|c|}{ Equipment planned } & \multirow{2}{*}{ References } \\
\hline & & & Yes (ID or label) & No & Yes (Project \#) & No & \\
\hline \multirow{4}{*}{$\begin{array}{l}242-\mathrm{A} \text { to } \\
\text { AW-106 } \\
(1998-2010)\end{array}$} & $\begin{array}{l}\text { 242-A to } 2 \text {-in. line SL-167 to } \\
\text { Valve Pit AW-B nozzle R3 }\end{array}$ & 2-in. slurry transfer line & Yes (2-in. SL-167-M2S) & & N/A & & H-14-020802 SH 5 \\
\hline & $\begin{array}{l}\text { Valve Pit AW-B nozzle R3 } \\
\text { via flex jumper to Valve Pit } \\
\text { AW-B nozzle R9 }\end{array}$ & $\begin{array}{l}\text { flex jumper from nozzle } \\
\text { R3 to nozzle R9 }\end{array}$ & $\begin{array}{l}\text { Yes (flex jumper shown } \\
\text { on routing board, no id } \\
\text { number) }\end{array}$ & & N/A & & Routing Board \\
\hline & $\begin{array}{l}\text { Valve Pit nozzle R9 line 2-in. } \\
\text { SL-166 to AW } 106 \text { Pump Pit } \\
\text { AW-06A nozzle B }\end{array}$ & 2-in. slurry transfer line & Yes (2-in. SL-166-M25) & & N/A & & H-14-020802 SH 3 \\
\hline & $\begin{array}{l}\text { Pump pit AW-06A nozzle B } \\
\text { to slurry distributor and into } \\
\text { Tank 106-AW }\end{array}$ & $\begin{array}{l}\text { Jumper from nozzle B to } \\
\text { siurry distributor }\end{array}$ & Yes & & N/A & & H-14-020802 SH 3 \\
\hline \multirow{2}{*}{$\begin{array}{l}\text { AW-102 to } \\
\text { 242-A (1998- } \\
2010)\end{array}$} & $\begin{array}{l}\text { Pump P-007 to nozzle B } \\
\text { (AW-102, pump pit AW-02E) }\end{array}$ & $\begin{array}{l}\text { Jumper or manifold from } \\
\text { P-007 to nozzle B }\end{array}$ & Yes & & N/A & & H-14-020080 SH 2 \\
\hline & $\begin{array}{l}\text { Nozzle B to 3-in. line SN-269 } \\
\text { to } 242-\mathrm{A}\end{array}$ & 3-in. transfer line & Yes & & N/A & & H-14-020080 SH 2 \\
\hline \multirow[t]{4}{*}{$\begin{array}{l}\text { AZ-101 to } \\
\text { AW-105 } \\
(2000-2001)\end{array}$} & $\begin{array}{l}\text { Pump P-AZ-101 to nozzle U6 } \\
\text { (AZ-101, pump pit AZ-01A) }\end{array}$ & $\begin{array}{l}\text { Jumper or manifold from } \\
\text { pump P-AZ-101 to nozzle } \\
\text { U6 }\end{array}$ & & No & Yes (W-314) & & $\begin{array}{l}\text { W-314 Alternatives } \\
\text { Analysis }\end{array}$ \\
\hline & $\begin{array}{l}\text { Nozzle U6 to } 3 \text {-in. line } \\
\text { AZ01A/NVP to valve pit } \\
\text { NVP nozzle B }\end{array}$ & 3-in. transfer line & & No & Yes (W-314) & & $\begin{array}{l}\text { W-314 Alternatives } \\
\text { Analysis }\end{array}$ \\
\hline & $\begin{array}{l}\text { Valve Pit NVP nozzle B to } \\
\text { nozzle E }\end{array}$ & $\begin{array}{l}\text { Jumper or manifold from } \\
\text { nozzle B to nozzle } \mathrm{E}\end{array}$ & & No & Yes (W-314) & & $\begin{array}{l}\text { W-314 Alternatives } \\
\text { Analysis }\end{array}$ \\
\hline & $\begin{array}{l}\text { Valve Pit NVP nozzle E to } \\
\text { 3-in. line NVP/APVP to } \\
\text { 241-AP valve pit nozzle } 15\end{array}$ & 3-in, transfer line & & No & Yes (W-314) & & $\begin{array}{l}\text { W-314 Alternatives } \\
\text { Analysis }\end{array}$ \\
\hline
\end{tabular}


Table A-2. Equipment Availability Matrix.

\begin{tabular}{|c|c|c|c|c|c|c|c|}
\hline \multirow{2}{*}{ Transfer } & \multirow{2}{*}{ Transfer route } & \multirow{2}{*}{ Equipment needs } & \multicolumn{2}{|c|}{ Equipment installed } & \multicolumn{2}{|c|}{ Equipment planned } & \multirow{2}{*}{ References } \\
\hline & & & Yes (ID or label) & No & Yes (Project \#) & No & \\
\hline \multirow{7}{*}{$\begin{array}{l}\text { AZ-101 to } \\
\text { AW-105 } \\
\text { (2000-2001) } \\
\text { (Continued) }\end{array}$} & $\begin{array}{l}\text { 241-AP valve pit nozzle } 15 \text { to } \\
\text { nozzle } 14\end{array}$ & $\begin{array}{l}\text { Jumper or manifold from } \\
\text { nozzle } 15 \text { to } 14\end{array}$ & & No & Yes (W-314) & & $\begin{array}{l}\text { W-314 Alternatives } \\
\text { Analysis }\end{array}$ \\
\hline & $\begin{array}{l}\text { 241-AP valve pit nozzle } 14 \text { to } \\
\text { 3-in. line SN-609 to pump pit } \\
\text { AW-02A nozzle V }\end{array}$ & 3-in. transfer line & Yes & & N/A & & $\begin{array}{c}\mathrm{H}-14-020802, \mathrm{Sh} 2 \\
:\end{array}$ \\
\hline & $\begin{array}{l}\text { Pump pit AW-02A nozzle } V \\
\text { to nozzle } J\end{array}$ & $\begin{array}{l}\text { Jumper or manifold from } \\
\text { nozzle } V \text { to nozzle } J\end{array}$ & Yes & & N/A & & H-14-020802, Sh 2 \\
\hline & $\begin{array}{l}\text { Pump pit AW-02A nozzle J to } \\
\text { 3-in. line SN-267 to 241-AW- } \\
\text { A valve pit nozzle L1 }\end{array}$ & 3-in. transfer line & Yes & & N/A & & H-14-020802, Sh 2 \\
\hline & $\begin{array}{l}\text { 241-AW-A valve pit nozzle } \\
\text { L1 to nozzle L15 }\end{array}$ & $\begin{array}{l}\text { Jumper or manifold from } \\
\text { nozzle L1 to L15 }\end{array}$ & & No & & No & H-14-020802 SH 4 \\
\hline & $\begin{array}{l}\text { 241-AW-A valve pit nozzle } \\
\text { L15 to 3-in. line SN-265 to } \\
\text { AW-105 pump pit AW-05A } \\
\text { nozzle A }\end{array}$ & 3-in. transfer line & Yes & & N/A & & H-14-020802 SH 4 \\
\hline & $\begin{array}{l}\text { Pump pit AW-05A nozzle } \mathrm{A} \\
\text { to tank return nozzle } \mathbf{G}\end{array}$ & $\begin{array}{l}\text { Jumper or manifold from } \\
\text { nozzle A to nozzle } G\end{array}$ & Yes & & N/A & & H-14-020802 SH 1 \\
\hline \multirow{2}{*}{$\begin{array}{l}\text { AN-103 to } \\
\text { AW-104 } \\
(2006)\end{array}$} & $\begin{array}{l}\text { SN pump P-007 to nozzle A } \\
\text { (AN-103, pump pit-AN-03A) }\end{array}$ & $\begin{array}{l}\text { Jumper or manifold from } \\
\text { P-007 to nozzle A }\end{array}$ & & No & Yes (W-211) & & $\begin{array}{l}\text { WHC-SD-W211- } \\
\text { TDR-001 }\end{array}$ \\
\hline & $\begin{array}{l}\text { Nozzle A to } 3 \text {-in. line } \\
\text { SN-263 to } 241-\text { AN-B valve } \\
\text { pit nozzle R14 }\end{array}$ & 3-in. transfer line & Yes & & N/A & & H-14-020801 SH 2 \\
\hline
\end{tabular}


Table A-2. Equipment Availability Matrix.

\begin{tabular}{|c|c|c|c|c|c|c|c|}
\hline Transfer & Transfer route & Equipment needs & \multicolumn{2}{|c|}{ Equipment installed } & \multicolumn{2}{|c|}{ Equipment planned } & References \\
\hline \multirow{7}{*}{$\begin{array}{l}\text { AN-103 to } \\
\text { AW-104 } \\
(2006) \\
\text { (continued) }\end{array}$} & $\begin{array}{l}\text { 241-AN-B valve pit nozzle } \\
\text { R14 to nozzle R15 }\end{array}$ & $\begin{array}{l}\text { Jumper or manifold from } \\
\text { nozzle R14 to nozzle R15 }\end{array}$ & & No & Yes (W-314) & & ES-314E-M40 \\
\hline & $\begin{array}{l}\text { Pump pit AN-01A nozzle A } \\
\text { to nozzle D }\end{array}$ & $\begin{array}{l}\text { Jumper or manifold from } \\
\text { nozzle } A \text { to nozzle } D\end{array}$ & & No & Yes (W-314) & & $\begin{array}{l}\text { W-314 Alternatives } \\
\text { Analysis }\end{array}$ \\
\hline & $\begin{array}{l}\text { Pump pit AN-01A nozzle D } \\
\text { to } 3 \text {-in, line AN101A/NVP to } \\
\text { valve pit NVP nozzle A }\end{array}$ & 3-in. transfer line & & No & Yes (W-314) & & $\begin{array}{l}\text { W-314 Alternatives } \\
\text { Analysis }\end{array}$ \\
\hline & $\begin{array}{l}\text { Valve pit NVP nozzle E to } \\
\text { 3-in. line NVP/APVP to } 241 \text { - } \\
\text { AP valve pit nozzle } 15\end{array}$ & 3-in. transfer line & . & No & Yes (W-314) & & $\begin{array}{l}\text { W-314 Alternatives } \\
\text { Analysis }\end{array}$ \\
\hline & $\begin{array}{l}\text { AP valve pit nozzle } 15 \text { to } \\
\text { nozzle } 13\end{array}$ & $\begin{array}{l}\text { Jumper or manifold from } \\
\text { nozzle } 15 \text { to nozzle } 13\end{array}$ & & No & Yes (W-314) & & $\begin{array}{l}\text { W-314 Alternatives } \\
\text { Analysis }\end{array}$ \\
\hline & $\begin{array}{l}\text { AP valve pit nozzle } 13 \text { to } \\
\text { 3-in. line SN-610 to pump pit } \\
\text { AW-02A nozzle U }\end{array}$ & 3-in. transfer line & Yes & & N/A & & H-14-020803 SH 5 \\
\hline & $\begin{array}{l}\text { Pump pit AW-02A nozzle } U \\
\text { to nozzle } \mathrm{H}\end{array}$ & $\begin{array}{l}\text { Jumper or manifold from } \\
\text { nozzle } \mathbf{U} \text { to nozzle } \mathbf{H}\end{array}$ & Yes & & N/A & & H-14-020802 SH 2 \\
\hline
\end{tabular}




\begin{tabular}{|c|c|c|c|c|c|c|c|}
\hline 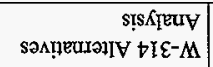 & & $(\sharp I \varepsilon-M) \operatorname{se} X$ & $\mathrm{oN}_{\mathrm{N}}$ & & 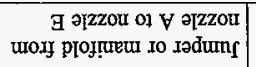 & 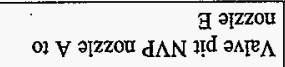 & \\
\hline 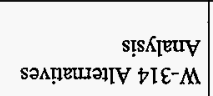 & & $(t I \varepsilon-M) s \partial \alpha$ & oN & & 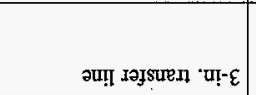 & 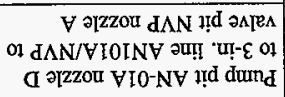 & \\
\hline 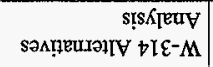 & & $(\downarrow I \varepsilon-M) s \partial X$ & $\mathrm{ON}$ & & 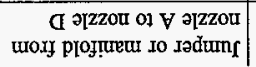 & 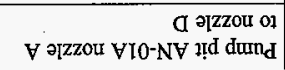 & \\
\hline $9 \mathrm{HS} \mathrm{I080Z0॰I-H}$ & & $\mathrm{V} / \mathrm{N}$ & & ${ }^{s} \boldsymbol{X}$ & 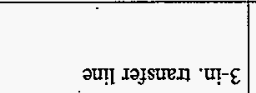 & 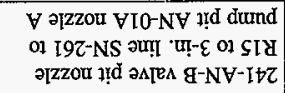 & \\
\hline $0 \triangleright W-G+I \varepsilon-S A$ & & $(t I \varepsilon-M) \operatorname{s} 2 \lambda$ & oN & & 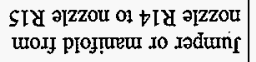 & 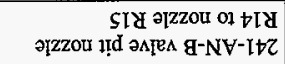 & \\
\hline$z$ HS I080Z0-trI-H & & $\mathrm{V} / \mathrm{N}$ & & saX & 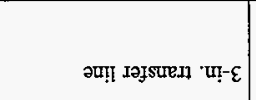 & 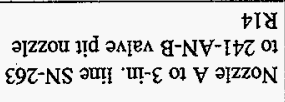 & \\
\hline $\begin{array}{r}\text { I00-8GJ } \\
-\operatorname{IIZM}-\mathrm{QS}-\supset \mathrm{HM}\end{array}$ & & $(I I Z M) \operatorname{sex}$ & $\mathrm{oN}_{\mathrm{N}}$ & & 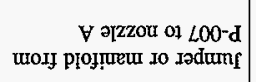 & 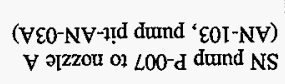 & $\begin{array}{r}(S 00 Z) \\
\varepsilon 0 I-M V \\
\text { ol } \varepsilon 0 I-N V\end{array}$ \\
\hline S HS $208020-\not[-H$ & & $\mathbf{V} / \mathrm{N}$ & & $\operatorname{sex}$ & 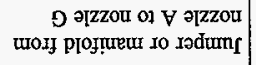 & 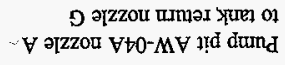 & \\
\hline$\downarrow \mathrm{HS} \mathrm{Z080Z0-tI-H}$ & & $\forall / N$ & & $s o x$ & 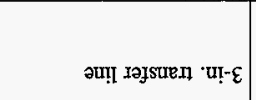 & 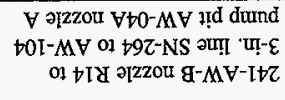 & (manumuron) \\
\hline$\downarrow$ HS z080z0- $\downarrow \tau-H$ & ON & & oN & & 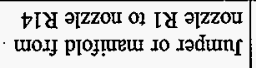 & 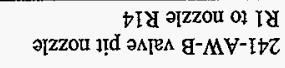 & $\begin{array}{l}\text { tOI-MV } \\
\text { of EOI-NV }\end{array}$ \\
\hline \multirow{2}{*}{ 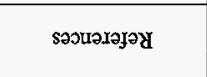 } & $\mathrm{ON}_{\mathrm{N}}$ & $(\# 10 \partial ! 0 I d) s \partial \lambda$ & $\mathrm{ON}$ & 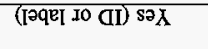 & \multirow[t]{2}{*}{ spəə๘ quəurd!̣nb̆ } & \multirow[t]{2}{*}{ əInOI IOJSUREIJ } & \multirow[t]{2}{*}{ 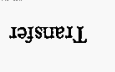 } \\
\hline & \multicolumn{2}{|c|}{ 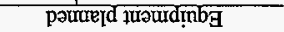 } & \multicolumn{2}{|c|}{ 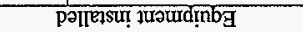 } & & & \\
\hline
\end{tabular}


Table A-2. Equipment Availability Matrix.

\begin{tabular}{|c|c|c|c|c|c|c|c|}
\hline Transfer & Transfer route & Equipment needs & \multicolumn{2}{|c|}{ Equipment installed } & \multicolumn{2}{|c|}{ Equipment planned } & References \\
\hline \multirow{6}{*}{$\begin{array}{l}\text { AN-103 to } \\
\text { AW-103 } \\
(2005) \\
\text { (continued) }\end{array}$} & $\begin{array}{l}\text { Valve pit NVP nozzle E to } \\
\text { 3-in. line NVP/APVP to } 241 \text { - } \\
\text { AP valve pit nozzle } 15\end{array}$ & 3-in. transfer line & & No & Yes (W-314) & & $\begin{array}{l}\text { W-314 Alternatives } \\
\text { Analysis }\end{array}$ \\
\hline & $\begin{array}{l}\text { AP valve pit nozzle } 14 \text { to } \\
3 \text {-in. line SN-609 to pump pit } \\
\text { AW-02A nozzle V }\end{array}$ & 3-in. transfer line & Yes & & N/A & & H-14-020803 SH 5 \\
\hline & $\begin{array}{l}\text { Pump pit AW-02A nozzle V } \\
\text { to nozzle } \mathrm{J}\end{array}$ & $\begin{array}{l}\text { Jumper or manifold from } \\
\text { nozzle } V \text { to nozzle } J\end{array}$ & Yes & & N/A & & H-14-020802 SH 2 \\
\hline & $\begin{array}{l}\text { 241-AW-A valve pit nozzle } \\
\text { L1 to nozzle L14 }\end{array}$ & $\begin{array}{l}\text { Jumper or manifold from } \\
\text { nozzle L1 to nozzle L14 }\end{array}$ & & No & & No & H-14-020802 SH 4 \\
\hline & $\begin{array}{l}\text { 241-AW-A nozzle L14 to } \\
\text { 3-in. line SN-263 to AW-103 } \\
\text { pump pit AW-03A nozzle A }\end{array}$ & 3-in. transfer line & Yes & & N/A & & H-14-020802 SH 4 \\
\hline & $\begin{array}{l}\text { Pump pit AW-03A nozzle } A \\
\text { to tank return nozzle } \mathrm{G}\end{array}$ & $\begin{array}{l}\text { Jumper or manifold from } \\
\text { nozzle } A \text { to nozzle } G\end{array}$ & & No & & No & H-14-020802 SH 5 \\
\hline $\begin{array}{l}\text { AW-106 to } \\
\text { AP-101 } \\
\text { (1998-1999) }\end{array}$ & $\begin{array}{l}\text { SN pump P-017 to nozzle } A \\
\text { (AW-106, pump pit-06A) }\end{array}$ & $\begin{array}{l}\text { Jumper or manifold from } \\
\text { P-017 to nozzle A }\end{array}$ & & No & & No & H-14-020802 SH 3 \\
\hline
\end{tabular}


Table A-2. Equipment Availability Matrix.

\begin{tabular}{|c|c|c|c|c|c|c|c|}
\hline \multirow{2}{*}{ Transfer } & \multirow{2}{*}{ Transfer route } & \multirow{2}{*}{ Equipment needs } & \multicolumn{2}{|c|}{ Equipment installed } & \multicolumn{2}{|c|}{ Equipment planned } & \multirow{2}{*}{ References } \\
\hline & & & Yes (ID or label) & No & Yes (Project \#) & No & \\
\hline \multirow{7}{*}{$\begin{array}{l}\text { AW-106 to } \\
\text { AP-101 } \\
\text { (1998-1999) } \\
\text { (Continued) }\end{array}$} & $\begin{array}{l}\text { 241-AW-B valve pit nozzle } \\
\text { R15 to nozzle } \mathrm{R} 1\end{array}$ & $\begin{array}{l}\text { Jumper or manifold from } \\
\text { nozzle R15 to nozzle R1 }\end{array}$ & Yes & & N/A & & Routing Board \\
\hline & $\begin{array}{l}\text { 241-AW-B valve pit nozzle } \\
\text { R1 to 3-in. line SN-268 to } \\
\text { AW-102 pump pit-AW-02A } \\
\text { nozzle A }\end{array}$ & 3-in. transfer line & Yes & & N/A & & H-14-020802 SH 5 \\
\hline & $\begin{array}{l}\text { Pump pit-AW-02A nozzle } \mathrm{H} \\
\text { to nozzle } \mathrm{U}\end{array}$ & $\begin{array}{l}\text { Jumper or manifold from } \\
\text { nozzle } H \text { to nozzle } U\end{array}$ & Yes & & N/A & & H-14-020802 SH 2 \\
\hline & $\begin{array}{l}\text { Pump pit-AW-02A nozzle U } \\
\text { to } 3 \text {-in. line SN-610 to } 241- \\
\text { AP valve pit nozzle } 13\end{array}$ & 3-in. transfer line & Yes & & N/A & & H-14-020802 SH 2 \\
\hline & $\begin{array}{l}\text { 241-AP valve pit nozzle } 13 \text { to } \\
\text { nozzle } 18\end{array}$ & $\begin{array}{l}\text { Jumper or manifold from } \\
\text { nozzle } 14 \text { to nozzle } 18\end{array}$ & Yes & & N/A & & H-14-020803 Sh 5 \\
\hline & $\begin{array}{l}\text { 241-AP valve pit nozzle } 18 \text { to } \\
\text { 3' line SN-611 to AP-101 } \\
\text { pump pit-AP-01A nozzle A }\end{array}$ & 3-in. transfer line & Yes & & N/A & & H-14-020803 Sh 5 \\
\hline & $\begin{array}{l}\text { Pump pit-AP-01A nozzle A to } \\
\text { tank return nozzle E }\end{array}$ & $\begin{array}{l}\text { Jumper or manifold from } \\
\text { nozzle A to nozzle E }\end{array}$ & Yes & & N/A & & H-14-020803 Sh 1 \\
\hline \multirow{3}{*}{$\begin{array}{l}\text { AN-105 to } \\
\text { AW-102 } \\
(2003-2009)\end{array}$} & $\begin{array}{l}\text { SN pump P-013 to nozzle A } \\
\text { (AN-105, pump pit-AN-05A) }\end{array}$ & $\begin{array}{l}\text { Jumper or manifold from } \\
\text { P-013 to nozzle } A\end{array}$ & & No & Yes (W211) & & $\begin{array}{l}\text { WHC-SD-W211- } \\
\text { TDR-001 }\end{array}$ \\
\hline & $\begin{array}{l}\text { Nozzle A to 3-in. line } \\
\text { SN-265 to } 241-\text { AN-A valve } \\
\text { pit nozzle L16 }\end{array}$ & 3-in. transfer line & Yes & & N/A & & H-14-020801 SH 2 \\
\hline & $\begin{array}{l}\text { 241-AN-A valve pit nozzle } \\
\text { L16 to nozzle L19 }\end{array}$ & $\begin{array}{l}\text { Jumper or manifold from } \\
\text { nozzle L16 to nozzle L19 }\end{array}$ & & No & Yes (W-314) & & ES-314E-M40 \\
\hline
\end{tabular}


Table A-2. Equipment Availability Matrix.

\begin{tabular}{|c|c|c|c|c|c|c|c|}
\hline \multirow{2}{*}{ Transfer } & \multirow{2}{*}{ Transfer route } & \multirow{2}{*}{ Equipment needs } & \multicolumn{2}{|c|}{ Equipment installed } & \multicolumn{2}{|c|}{ Equipment planned } & \multirow{2}{*}{ References } \\
\hline & & & Yes (ID or label) & No & Yes (Project \#) & No & \\
\hline \multirow[t]{10}{*}{$\begin{array}{l}\text { AN-105 to } \\
\text { AW-102 } \\
(2003-2009) \\
\text { (continued) }\end{array}$} & $\begin{array}{l}\text { 241-AN-A valve pit nozzle } \\
\text { L19 to } 3 \text {-in. line SN-268 to } \\
\text { 241-AN-B valve pit nozzle } \\
\text { R19 }\end{array}$ & 3-in. transfer line & Yes & & N/A & & H-14-020801 SH 5 \\
\hline & $\begin{array}{l}\text { 241-AN-B valve pit nozzle } \\
\text { R19 to nozzle R15 }\end{array}$ & $\begin{array}{l}\text { Jumper or manifold from } \\
\text { nozzle R14 to nozzle R15 }\end{array}$ & & No & Yes (W-314) & & ES-314E-M40 \\
\hline & $\begin{array}{l}\text { 241-AN-B valve pit nozzle } \\
\text { R15 to 3-in. line SN-261 to } \\
\text { pump pit AN-01A nozzle A }\end{array}$ & 3-in. transfer line & Yes & & N/A & & H-14-020801 SH 6 \\
\hline & $\begin{array}{l}\text { Pump pit AN-01A nozzle A } \\
\text { to nozzle D }\end{array}$ & $\begin{array}{l}\text { Jumper or manifold from } \\
\text { nozzle A to nozzle D }\end{array}$ & & No & Yes (W-314) & & $\begin{array}{l}\text { W-314 Alternatives } \\
\text { Anaiysis }\end{array}$ \\
\hline & $\begin{array}{l}\text { Pump pit AN-01A nozzle D } \\
\text { to } 3 \text {-in. line AN101A/NVP to } \\
\text { valve pit NVP nozzle A }\end{array}$ & 3-in. transfer line & & No & Yes (W-314) & & $\begin{array}{l}\text { W-314 Alternatives } \\
\text { Analysis }\end{array}$ \\
\hline & $\begin{array}{l}\text { Valve pit NVP nozzle A to } \\
\text { nozzle E }\end{array}$ & $\begin{array}{l}\text { Jumper or manifold from } \\
\text { nozzle A to nozzle } E\end{array}$ & & No & Yes (W-314) & & $\begin{array}{l}\text { W-314 Alternatives } \\
\text { Analysis }\end{array}$ \\
\hline & $\begin{array}{l}\text { Valve pit NVP nozzle E to } \\
\text { 3-in. line NVP/APVP to } 241 \text { - } \\
\text { AP valve pit nozzle } 15\end{array}$ & 3-in. transfer line & & No & Yes (W-314) & & $\begin{array}{l}\text { W-314 Alternatives } \\
\text { Analysis }\end{array}$ \\
\hline & $\begin{array}{l}\text { AP valve pit nozzle } 15 \text { to } \\
\text { nozzle } 13\end{array}$ & $\begin{array}{l}\text { Jumper or manifold from } \\
\text { nozzle } 15 \text { to nozzle } 13\end{array}$ & & No & Yes (W-314) & & $\begin{array}{l}\text { W-314 Alternatives } \\
\text { Analysis }\end{array}$ \\
\hline & $\begin{array}{l}\text { AP valve pit nozzle } 13 \text { to } \\
\text { 3-in. line } S N-610 \text { to pump pit } \\
\text { AW-02A nozzle U }\end{array}$ & 3-in. transfer line & Yes & & N/A & & H-14-020803 SH 5 \\
\hline & $\begin{array}{l}\text { Pump pit AW-02A nozzle } U \\
\text { to tank return nozzle } \mathrm{L}\end{array}$ & $\begin{array}{l}\text { Jumper or manifold from } \\
\text { nozzle } U \text { to nozzle } L\end{array}$ & Yes & & N/A & & H-14-020802 SH 2 \\
\hline
\end{tabular}


Table A-2. Equipment Availability Matrix.

\begin{tabular}{|c|c|c|c|c|c|c|c|}
\hline \multirow{2}{*}{ Transfer } & \multirow{2}{*}{ Transfer route } & \multirow{2}{*}{ Equipment needs } & \multicolumn{2}{|c|}{ Equipment installed } & \multicolumn{2}{|c|}{ Equipment planned } & \multirow{2}{*}{ References } \\
\hline & & & Yes (ID or label) & No & Yes (Project \#) & No & \\
\hline \multirow{9}{*}{$\begin{array}{l}\text { AW-106 to } \\
\text { AP-107 } \\
(2000)\end{array}$} & $\begin{array}{l}\text { SN pump P-017 to nozzle A } \\
\text { (AW-106, pump pit-AW-06A) }\end{array}$ & $\begin{array}{l}\text { Jumper or manifold from } \\
\text { P-017 to nozzle A }\end{array}$ & . & No & & No & H-14-020802 SH 3 \\
\hline & $\begin{array}{l}\text { Nozzle A to } 3 \text {-in. line SN-266 } \\
\text { to } 241-A W-B \text { valve pit nozzle } \\
\text { R15 }\end{array}$ & 3-in. transfer line & Yes & & N/A & & $\mathrm{H}-14-020802 \mathrm{SH} 3$ \\
\hline & $\begin{array}{l}\text { 241-AW-B valve pit nozzle } \\
\text { R15 to nozzle R1 }\end{array}$ & $\begin{array}{l}\text { Jumper or manifold from } \\
\text { nozzle R15 to nozzle R1 }\end{array}$ & Yes & & N/A & & H-14-020802 SH 5 \\
\hline & $\begin{array}{l}\text { 241-AW-B valve pit nozzle } \\
\text { R1 to 3-in. line SN-268 to } \\
\text { AW-102 pump pit-AW-02A } \\
\text { nozzle } 14\end{array}$ & 3-in. transfer line & Yes & & N/A & & H-14-020802 SH 2 \\
\hline & $\begin{array}{l}\text { Pump pit-AW-02A nozzle } \mathrm{H} \\
\text { to nozzle } \mathrm{U}\end{array}$ & $\begin{array}{l}\text { Jumper or manifold from } \\
\text { nozzle } \mathrm{H} \text { to nozzle } \mathrm{U}\end{array}$ & Yes & & N/A & & H-14-020802 SH 2 \\
\hline & $\begin{array}{l}\text { Pump pit-AW-02A nozzle U } \\
\text { to } 3 \text {-in. line SN-610 to } 241 \text { - } \\
\text { AP-valve pit nozzle } 13\end{array}$ & 3-in. transfer line & Yes & & N/A & & H-14-020802 SH 2 \\
\hline & $\begin{array}{l}\text { 241-AP-valve pit nozzle } 13 \text { to } \\
\text { nozzle } 23\end{array}$ & $\begin{array}{l}\text { Jumper or manifold from } \\
\text { nozzle } 1 \text { to nozzle } 23\end{array}$ & Yes & & N/A & & H-14-020803 SH 5 \\
\hline & $\begin{array}{l}\text { 241-AP-valve pit nozzle } 23 \text { to } \\
\text { 3-in. line SN-617 to AP-107 } \\
\text { pump pit-AP-07A nozzle A }\end{array}$ & 3-in. transfer line & Yes & & N/A & & H-14-020803 SH 5 \\
\hline & $\begin{array}{l}\text { Pump pit-AP-07A nozzle A to } \\
\text { tank teturn nozzle E }\end{array}$ & $\begin{array}{l}\text { Jumper or manifold from } \\
\text { nozzle A to nozzle E }\end{array}$ & Yes & & N/A & & H-14-020803 SH 2 \\
\hline $\begin{array}{l}\text { AW-106 to } \\
\text { AP-108 } \\
\text { (1999) }\end{array}$ & $\begin{array}{l}\text { SN pump P-017 to nozzle } A \\
\text { (AW-106, pump pit-AW-06A) }\end{array}$ & $\begin{array}{l}\text { Jumper or manifold from } \\
\text { P-017 to nozzle A }\end{array}$ & & No & & No & H-14-020802 SH 3 \\
\hline
\end{tabular}


Table A-2. Equipment Availability Matrix.

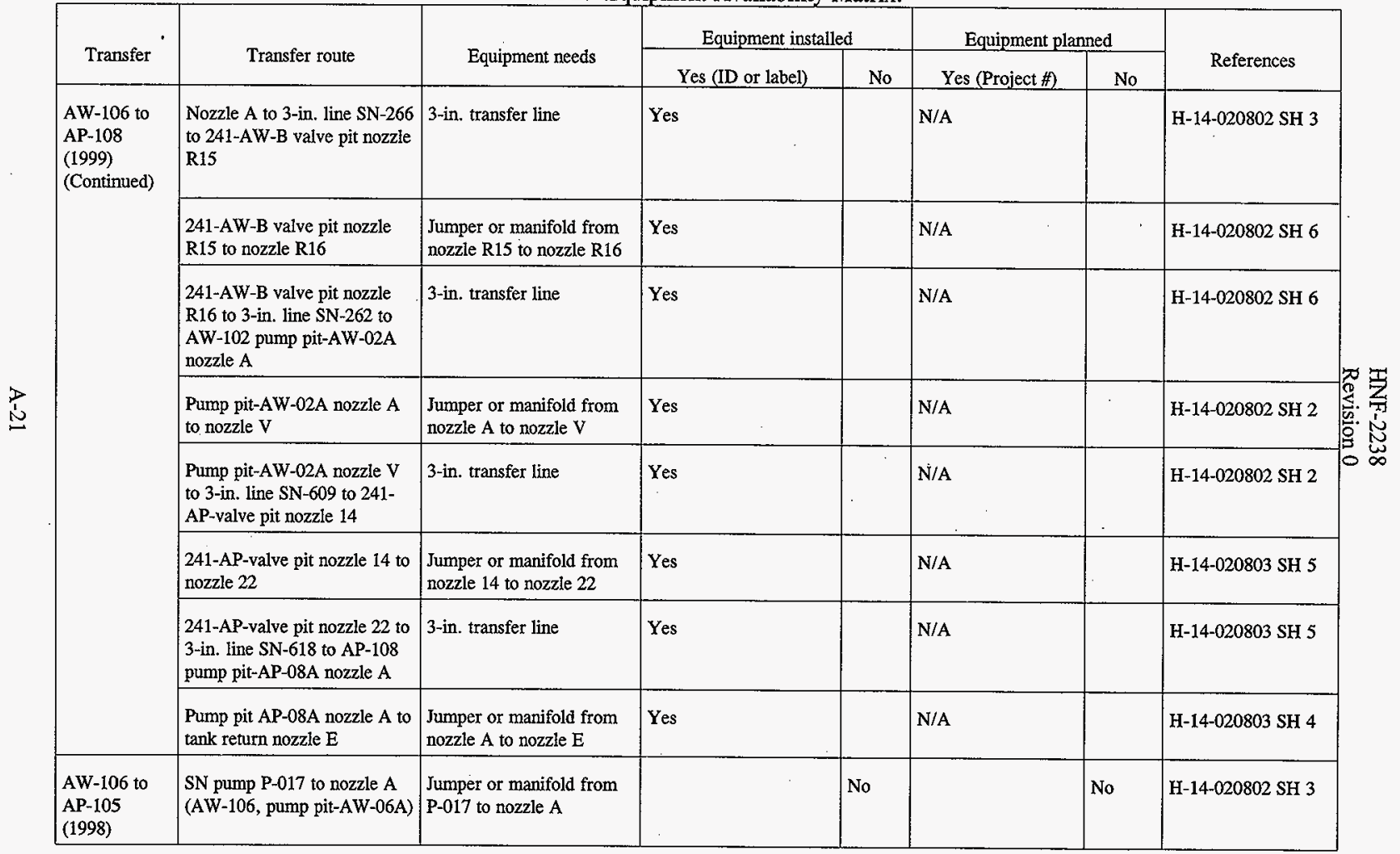


Table A-2. Equipment Availability Matrix.

\begin{tabular}{|c|c|c|c|c|c|c|c|}
\hline & \multirow{2}{*}{ Transfer route } & \multirow{2}{*}{ Equipment needs } & \multicolumn{2}{|c|}{ Equipment installed } & \multicolumn{2}{|c|}{ Equipment planned } & \multirow{2}{*}{ References } \\
\hline 1 ransier & & & Yes (ID or labèl) & No & Yes (Project \#) & No & \\
\hline \multirow{8}{*}{$\begin{array}{l}\text { AW-106 to } \\
\text { AP-105 } \\
\text { (1998) } \\
\text { (Continued) }\end{array}$} & $\begin{array}{l}\text { Nozzle A to 3-in. line SN-266 } \\
\text { to } 241 \text {-AW-B valve pit nozzle } \\
\text { R15 }\end{array}$ & 3-in. transfer line & Yes & & N/A & & H-14-020802 SH 3 \\
\hline & $\begin{array}{l}\text { 241-AW-B valve pit nozzle } \\
\text { R15 to nozzle R1 }\end{array}$ & $\begin{array}{l}\text { Jumper or manifold from } \\
\text { nozzle R15 to nozzle R1 }\end{array}$ & Yes & & N/A & & H-14-020802 SH 6 \\
\hline & $\begin{array}{l}\text { 241-AW-B valve pit nozzle } \\
\text { R1 to } 3 \text {-in. line SN-268 to } \\
\text { AW-102 pump pit-AW-02A } \\
\text { nozzle } \mathrm{H}\end{array}$ & 3-in. transfer line & Yes & & N/A & & H-14-020802 SH 6 \\
\hline & $\begin{array}{l}\text { Pump pit-AW-02A nozzle } \mathrm{H} \\
\text { to nozzle } \mathrm{U}\end{array}$ & $\begin{array}{l}\text { Jumper or manifold from } \\
\text { nozzle } H \text { to nozzle } U\end{array}$ & Yes & & N/A & & H-14-020802 SH 2 \\
\hline & $\begin{array}{l}\text { Pump pit-AW-02A nozzle } \mathrm{H} \\
\text { to } 3 \text {-in. line SN-610 to } 241- \\
\text { AP-valve pit nozzle } 13\end{array}$ & 3-in. transfer line & Yes & & N/A & & H-14-020802 SH 2 \\
\hline & $\begin{array}{l}\text { 241-AP-valve pit nozzle } 13 \text { to } \\
\text { nozzle } 24\end{array}$ & $\begin{array}{l}\text { Jumper or manifold from } \\
\text { nozzle } 13 \text { to nozzle } 24\end{array}$ & Yes & & N/A & & H-14-020803 SH 5 \\
\hline & $\begin{array}{l}\text { 241-AP-valve pit nozzle } 24 \text { to } \\
\text { 3-in. line SN-615 to AP-105 } \\
\text { pump pit-AP05A nozzle A }\end{array}$ & 3-in. transfer line & Yes & & N/A & & H-14-020803 SH 5 \\
\hline & $\begin{array}{l}\text { Pump pit-AP-05A nozzle A to } \\
\text { tank return nozzle E }\end{array}$ & $\begin{array}{l}\text { Jumper or manifold from } \\
\text { nozzle A to nozzle E }\end{array}$ & Yes & & N/A & & H-14-020803 SH 2 \\
\hline \multirow{2}{*}{$\begin{array}{l}\text { AW-104 to } \\
\text { AP-107 } \\
\text { (1998) }\end{array}$} & $\begin{array}{l}\text { SN pump P-011 to nozzle A } \\
\text { (AW-104, pump pit-AW-04A) }\end{array}$ & $\begin{array}{l}\text { Jumper or manifold from } \\
\text { P-011 to nozzle A }\end{array}$ & Yes (Pump failed) & & N/A & & H-14-020802 SH 3 \\
\hline & $\begin{array}{l}\text { Nozzle A to } 3 \text {-in. line SN-264 } \\
\text { to } 241-A W-B \text { valve pit nozzle } \\
\text { R14 }\end{array}$ & 3-in. transfer line & Yes & & N/A & & H-14-020802 SH 3 \\
\hline
\end{tabular}


Table A-2. Equipment Availability Matrix.

\begin{tabular}{|c|c|c|c|c|c|c|c|}
\hline \multirow{2}{*}{ Transfer } & \multirow{2}{*}{ Transfer route } & \multirow{2}{*}{ Equipment needs } & \multicolumn{2}{|c|}{ Equipment installed } & \multicolumn{2}{|c|}{ Equipment planned } & \multirow{2}{*}{ References } \\
\hline & & & Yes (ID or label) & No & Yes (Project \#) & No & \\
\hline \multirow{7}{*}{$\begin{array}{l}\text { AW-104 to } \\
\text { AP-107 } \\
\text { (1998) } \\
\text { (Continued) }\end{array}$} & $\begin{array}{l}\text { 241-AW-B valve pit nozzle } \\
\text { R14 to nozzle R1 }\end{array}$ & $\begin{array}{l}\text { Jumper or manifold from } \\
\text { nozzle R14 to nozzle RI }\end{array}$ & & No & . & No & H-14-020802 SH 4 \\
\hline & $\begin{array}{l}\text { 241-AW-B valve pit nozzle } \\
\text { R1 to } 3 \text {-in. line SN-268 to } \\
\text { AW-102 pump pit-AW-02A } \\
\text { nozzle H }\end{array}$ & 3-in. transfer line & Yes & & N/A & & H-14-020802 SH 2 \\
\hline & $\begin{array}{l}\text { Pump pit-AW-02A nozzle } \mathrm{H} \\
\text { to nozzle } \mathrm{U}\end{array}$ & $\begin{array}{l}\text { Jumper or manifold from } \\
\text { nozzle } \mathrm{H} \text { to nozzle } \mathrm{U}\end{array}$ & Yes & & N/A & & H-14-020802 SH 2 \\
\hline & $\begin{array}{l}\text { Pump pit-AW-02A nozzle U } \\
\text { to } 3 \text {-in. line SN-610 to } 241 \text { - } \\
\text { AP-valve pit nozzle } 14\end{array}$ & 3-in. transfer line & Yes & & N/A & & H-14-020802 SH 2 \\
\hline & $\begin{array}{l}\text { 241-AP-valve pit nozzle } 14 \text { to } \\
\text { nozzle } 23\end{array}$ & $\begin{array}{l}\text { Jumper or manifold from } \\
\text { nozzle } 14 \text { to nozzle } 23\end{array}$ & Yes & & N/A & & H-14-020803 SH 4 \\
\hline & $\begin{array}{l}\text { 241-AP-valve pit nozzle } 23 \text { to } \\
\text { 3-in. line SN-617 to AP-107 } \\
\text { pump pit-AP-07A nozzle A }\end{array}$ & 3-in. transfer line & Yes & & N/A & & H-14-020802 SH 4 \\
\hline & $\begin{array}{l}\text { Pump pit-AP-07A nozzle A to } \\
\text { tank return nozzle } \mathrm{E}\end{array}$ & $\begin{array}{l}\text { Jumper or manifold from } \\
\text { nozzle } \mathrm{A} \text { to nozzle } \mathrm{E}\end{array}$ & Yes & & N/A & & H-14-020802 SH 2 \\
\hline \multirow{3}{*}{$\begin{array}{l}\text { AP-101 to } \\
\text { AW-104 } \\
(1998)\end{array}$} & $\begin{array}{l}\text { SN pump P-001 to nozzle A } \\
\text { (AP-101, pump pit-AP-01A) }\end{array}$ & $\begin{array}{l}\text { Jumper or manifold from } \\
\text { P-001 to nozzle A }\end{array}$ & Yes & & N/A & & H-14-020803 SH 1 \\
\hline & $\begin{array}{l}\text { Pump pit-AP-01A nozzle A to } \\
\text { 3-in. line SN-611 to } 241-A P- \\
\text { valve pit nozzle } 18\end{array}$ & 3-in. transfer line & Yes & & N/A & & H-14-020803 SH 1 \\
\hline & $\begin{array}{l}\text { 241-AP-valve pit nozzle } 18 \text { to } \\
\text { nozzle } 13\end{array}$ & $\begin{array}{l}\text { Jumper or manifold from } \\
\text { nozzle } 18 \text { to nozzle } 13\end{array}$ & Yes & & N/A & & H-14-020803 SH 5 \\
\hline
\end{tabular}


Table A-2. Equipment Availability Matrix.

\begin{tabular}{|c|c|c|c|c|c|c|c|}
\hline \multirow{2}{*}{ Transfer } & \multirow{2}{*}{ Transfer route } & \multirow{2}{*}{ Equipment needs } & \multicolumn{2}{|c|}{ Equipment installed } & \multicolumn{2}{|c|}{ Equipment planned } & \multirow{2}{*}{ References } \\
\hline & & & Yes (ID or label) & No & Yes (Project \#) & No & \\
\hline \multirow{6}{*}{$\begin{array}{l}\text { AP-101 to } \\
\text { AW-104 } \\
\text { (1998) } \\
\text { (Continued) }\end{array}$} & $\begin{array}{l}\text { 241-AP-valve pit nozzle } 13 \text { to } \\
\text { 3-in. line SN-610 to AW-102 } \\
\text { pump pit-AW-02A nozzle U }\end{array}$ & 3-in. transfer line & Yes & & N/A & & H-14-020803 SH 5 \\
\hline & $\begin{array}{l}\text { Pump pit-AW-02A nozzle U } \\
\text { to nozzle } \mathrm{H}\end{array}$ & $\begin{array}{l}\text { Jumper or manifold from } \\
\text { nozzle V to nozzle A }\end{array}$ & Yes & & N/A & & $\mathrm{H}-14-020802 \mathrm{SH} 2$ \\
\hline & $\begin{array}{l}\text { Pump pit-AW-02A nozzle } \mathrm{H} \\
\text { to } 3 \text {-in. line } \mathrm{SN}-268 \text { to } 241- \\
\text { AW-B valve pit nozzle } \mathrm{R} 1\end{array}$ & 3-in. transfer line & Yes & & N/A & & H-14-020802 SH 2 \\
\hline & $\begin{array}{l}\text { 241-AW-B valve pit nozzle } \\
\text { R1 to nozzle R14 } \\
\end{array}$ & $\begin{array}{l}\text { Jumper or manifold from } \\
\text { nozzle R1 to nozzle R14 }\end{array}$ & & No & & No & H-14-020802 SH 5 \\
\hline & $\begin{array}{l}\text { 241-AW-B valve pit nozzie } \\
\text { R14 to 3-in. line SN-264 to } \\
\text { AW-104 pump pit-AW-04A } \\
\text { nozzle A }\end{array}$ & 3-in. transfer line & Yes & & N/A & & H-14-020802 SH 5 \\
\hline & $\begin{array}{l}\text { Pump pit-AW-04A nozzle A } \\
\text { to tank return nozzle G }\end{array}$ & $\begin{array}{l}\text { Jumper or manifold from } \\
\text { nozzle } A \text { to nozzle } G\end{array}$ & Yes & & N/A & & H-14-020802 SH 3 \\
\hline \multirow{3}{*}{$\begin{array}{l}\text { AP-101 to } \\
\text { AW-103 } \\
\text { (1998) }\end{array}$} & $\begin{array}{l}\text { SN pump P-001 to nozzle } A \\
\text { (AP-101, pump pit-AP-01A) }\end{array}$ & $\begin{array}{l}\text { Jumper or manifold from } \\
\text { P-001 to nozzle A }\end{array}$ & Yes & & N/A & & $\mathrm{H}-14-020803 \mathrm{SH} 1$ \\
\hline & $\begin{array}{l}\text { Pump pit-AP-01A nozzle A to } \\
\text { 3-in. line SN-611 to 241-AP- } \\
\text { valve pit nozzle } 18\end{array}$ & 3-in. transfer line & Yes & & N/A & & H-14-020803 SH 1 \\
\hline & $\begin{array}{l}\text { 241-AP-valve pit nozzle } 18 \text { to } \\
\text { nozzle } 14\end{array}$ & $\begin{array}{l}\text { Jumper or manifold from } \\
\text { nozzle } 18 \text { to nozzle } 14\end{array}$ & Yes & & $\mathrm{N} / \mathrm{A}$ & & H-14-020803 SH 5 \\
\hline
\end{tabular}


Table A-2. Equipment Availability Matrix.

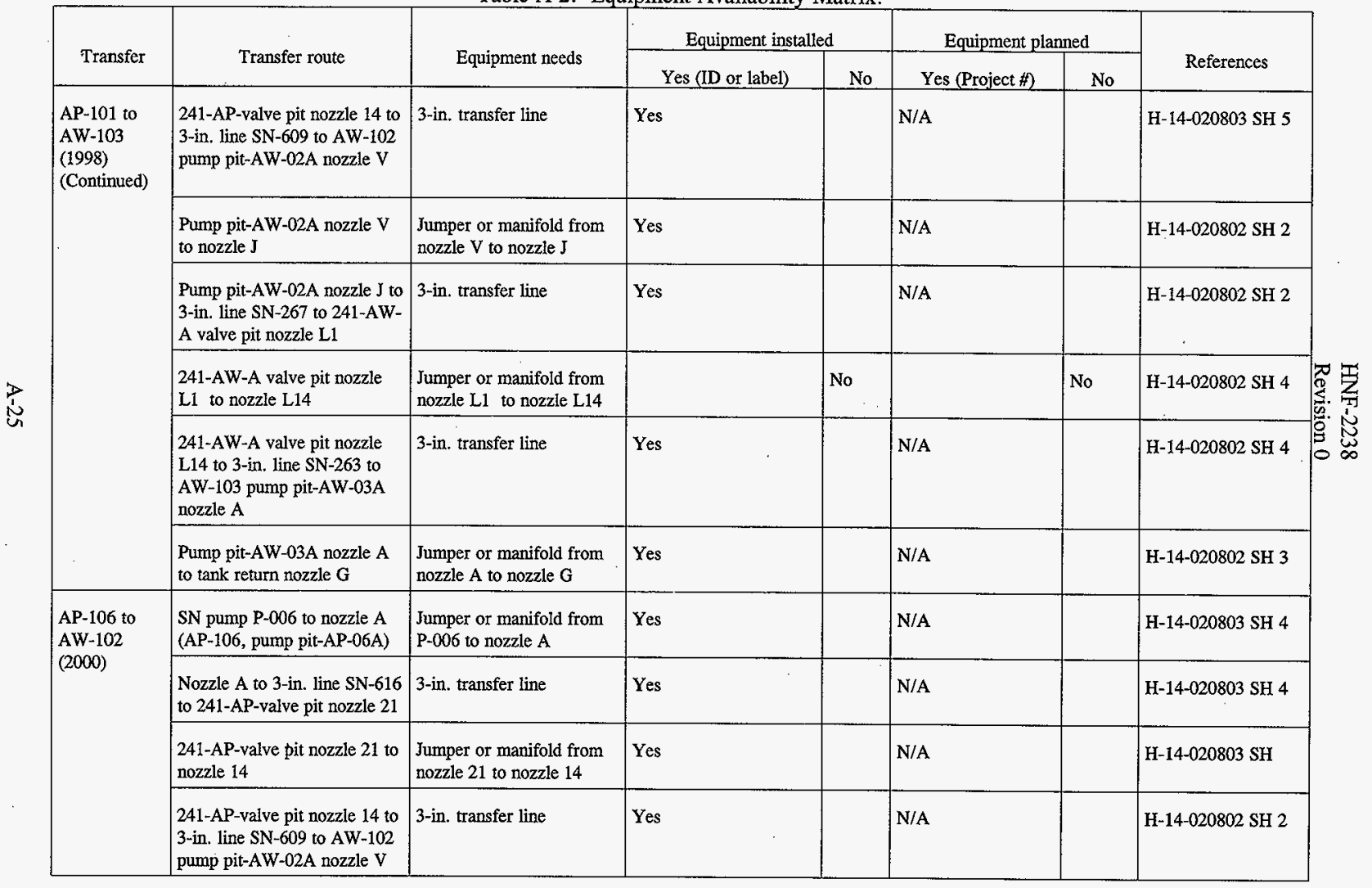


Table A-2. Equipment Availability Matrix.

\begin{tabular}{|c|c|c|c|c|c|c|c|}
\hline \multirow{2}{*}{ Transfer } & \multirow{2}{*}{ Transfer route } & \multirow{2}{*}{ Equipment needs } & \multicolumn{2}{|c|}{ Equipment installed } & \multicolumn{2}{|c|}{ Equipment planned } & \multirow{2}{*}{ References } \\
\hline & & & Yes (ID or label). & No & Yes (Project \#) & No & \\
\hline $\begin{array}{l}\text { AP-106 to } \\
\text { AW-102 } \\
(2000) \\
\text { (Continued) }\end{array}$ & $\begin{array}{l}\text { Pump pit-AW-02A nozzle } \mathrm{V} \\
\text { to tank return nozzle } \mathrm{L}\end{array}$ & $\begin{array}{l}\text { Jumper or manifold from } \\
\text { nozzle } V \text { to nozzle } L\end{array}$ & Yes & & N/A & & H-14-020802 SH 2 \\
\hline \multirow{5}{*}{$\begin{array}{l}\text { AP-103 to } \\
\text { AW-102 } \\
(1999)\end{array}$} & $\begin{array}{l}\text { SN pump P-003 to nozzle A } \\
\text { (AP-103, pump pit-AP-03A) }\end{array}$ & $\begin{array}{l}\text { Jumper or manifold from } \\
\text { P-003 to nozzle A }\end{array}$ & Yes & & N/A & & H-14-020803 SH 1 \\
\hline & $\begin{array}{l}\text { Nozzle A to } 3 \text {-in. line SN-613 } \\
\text { to } 241 \text {-AP-valve pit nozzle } 17\end{array}$ & 3-in. transfer line & Yes & & N/A & & H-14-020803 SH 4 \\
\hline & $\begin{array}{l}\text { 241-AP-valve pit nozzle } 17 \text { to } \\
\text { nozzle } 14\end{array}$ & $\begin{array}{l}\text { Jumper or manifold from } \\
\text { nozzle } 17 \text { to nozzle } 14\end{array}$ & Yes & & N/A & & H-14-020803 SH 5 \\
\hline & $\begin{array}{l}\text { 241-AP-valve pit nozzle } 14 \text { to } \\
\text { 3-in. line } S N-609 \text { to AW-102 } \\
\text { pump pit-AW-02A nozzle } V\end{array}$ & 3-in. transfer line & Yes & & N/A & & H-14-020803 SH 5 \\
\hline & $\begin{array}{l}\text { Pump pit-AW-02A nozzle V } \\
\text { to tank return nozzle } \mathrm{L}\end{array}$ & $\begin{array}{l}\text { Jumper or manifold from } \\
\text { nozzle } \mathrm{V} \text { to nozzle } \mathrm{L}\end{array}$ & Yes & & N/A & & H-14-020802 SH 2 \\
\hline \multirow[t]{4}{*}{$\begin{array}{l}\text { AP-107 to } \\
\text { AW-102 } \\
\text { (1998-1999) }\end{array}$} & $\begin{array}{l}\text { SN pump P-007 to nozzle A } \\
\text { (AP-107, pump pit-AP-07A) }\end{array}$ & $\begin{array}{l}\text { Jumper from P-007 to } \\
\text { nozzle A }\end{array}$ & Yes & & N/A & & H-14-020803 SH 2 \\
\hline & $\begin{array}{l}\text { Nozzle A to 3-in. line SN-617 } \\
\text { to } 241 \text {-AP-valve pit nozzle } 23\end{array}$ & 3-in. transfer line & Yes & & $\mathrm{N} / \mathrm{A}$ & & H-14-020803 SH 2 \\
\hline & $\begin{array}{l}\text { 241-AP-valve pit nozzle } 23 \text { to } \\
\text { nozzle } 14\end{array}$ & $\begin{array}{l}\text { Jumper or manifold from } \\
\text { nozzle } 23 \text { to nozzle } 14\end{array}$ & Yes & & N/A & & H-14-020803 SH 5 \\
\hline & $\begin{array}{l}\text { 241-AP-valve pit nozzle } 14 \text { to } \\
\text { 3-in. line SN-609 to AW-102 } \\
\text { pump pit-AW-02A nozzle V }\end{array}$ & 3-in. transfer line & Yes & & N/A & & H-14-020803 SH 5 \\
\hline
\end{tabular}


Table A-2. Equipment Availability Matrix.

\begin{tabular}{|c|c|c|c|c|c|c|c|}
\hline \multirow{2}{*}{ Transfer } & \multirow{2}{*}{ Transfer route } & \multirow{2}{*}{ Equipment needs } & \multicolumn{2}{|c|}{ Equipment installed } & \multicolumn{2}{|c|}{ Equipment planned } & \multirow{2}{*}{ References } \\
\hline & & & Yes (ID or label) & No & Yes (Project \#) & No & \\
\hline $\begin{array}{l}\text { AP-107 to } \\
\text { AW-102 } \\
\text { (1998-1999) } \\
\text { (continued) }\end{array}$ & $\begin{array}{l}\text { Pump pit-AW-02A nozzle V } \\
\text { to tank return nozzle } \mathrm{L}\end{array}$ & $\begin{array}{l}\text { Jumper or manifold from } \\
\text { nozzle } V \text { to nozzle } L\end{array}$ & Yes & & N/A & & H-14-020802 SH 2 \\
\hline \multirow{5}{*}{$\begin{array}{l}\text { AW-106 to } \\
\text { AW-102 } \\
(1998)\end{array}$} & $\begin{array}{l}\text { SN pump P-017 to nozzle } A \\
\text { (AW-106, pump pit-AW-06A) }\end{array}$ & $\begin{array}{l}\text { Jumper or manifold from } \\
\text { P-017 to nozzle A }\end{array}$ & & No & & No & H-14-020802 SH 3 \\
\hline & $\begin{array}{l}\text { Nozzle A to } 3 \text {-in. line SN-266 } \\
\text { to } 241 \text {-AW-B valve pit nozzle } \\
\text { R15 }\end{array}$ & 3-in. transfer line & Yes & & N/A & & H-14-020802 SH 3 \\
\hline & $\begin{array}{l}\text { 241-AW-B valve pit nozzle } \\
\text { R15 to nozzle R11 }\end{array}$ & $\begin{array}{l}\text { Jumper or manifold from } \\
\text { nozzle R15 to nozzle R11 }\end{array}$ & Yes & & N/A & & H-14-020802 SH 5 \\
\hline & $\begin{array}{l}\text { 241-AW-B valve pit nozzle } \\
\text { R1 to 3-in. line SN-268 to } \\
\text { AW-102 pump pit-AW-02A } \\
\text { nozzle } \mathrm{H}\end{array}$ & 3-in. transfer line & Yes & & N/A & & H-14-020802 SH 5 \\
\hline & $\begin{array}{l}\text { Pump pit-AW-02A nozzle } \mathrm{H} \\
\text { to tank return nozzle } \mathrm{N}\end{array}$ & $\begin{array}{l}\text { Jumper or manifold from } \\
\text { nozzle } \mathrm{H} \text { to nozzle } \mathrm{N}\end{array}$ & Yes & & N/A & & H-14-020802 SH 2 \\
\hline \multirow{3}{*}{$\begin{array}{l}\text { AW-106 to } \\
\text { AW-104 } \\
\text { (1999) }\end{array}$} & $\begin{array}{l}\text { SN pump P-017 to nozzle A } \\
\text { (AW-106, pump pit-AW-06A) }\end{array}$ & $\begin{array}{l}\text { Jumper or manifold from } \\
\text { P-017 to nozzle A }\end{array}$ & & No & & No & H-14-020802 SH 3 \\
\hline & $\begin{array}{l}\text { Nozzle A to 3-in. line SN-266 } \\
\text { to } 241 \text {-AW-B valve pit nozzle } \\
\text { R15 }\end{array}$ & 3-in. transfer line & Yes & & N/A & & H-14-020802 SH 3 \\
\hline & $\begin{array}{l}\text { 241-AW-B valve pit nozzle } \\
\text { R15 to nozzle R14 }\end{array}$ & $\begin{array}{l}\text { Jumper or manifold from } \\
\text { nozzle R15 to nozzle R14 }\end{array}$ & & No & & No & H-14-020802 SH 5 \\
\hline
\end{tabular}


Table A-2. Equipment Availability Matrix.

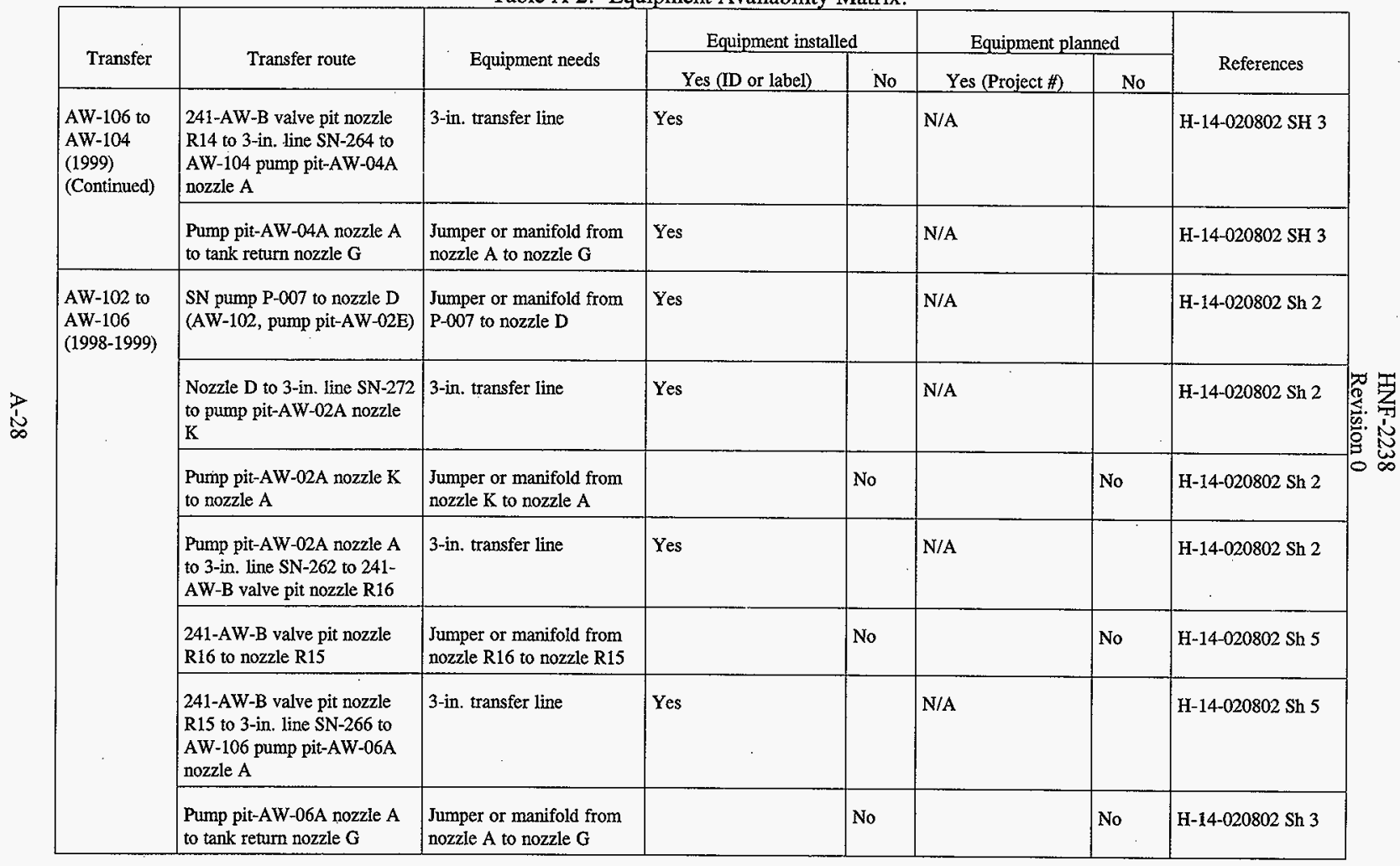


Table A-2. Equipment Availability Matrix.

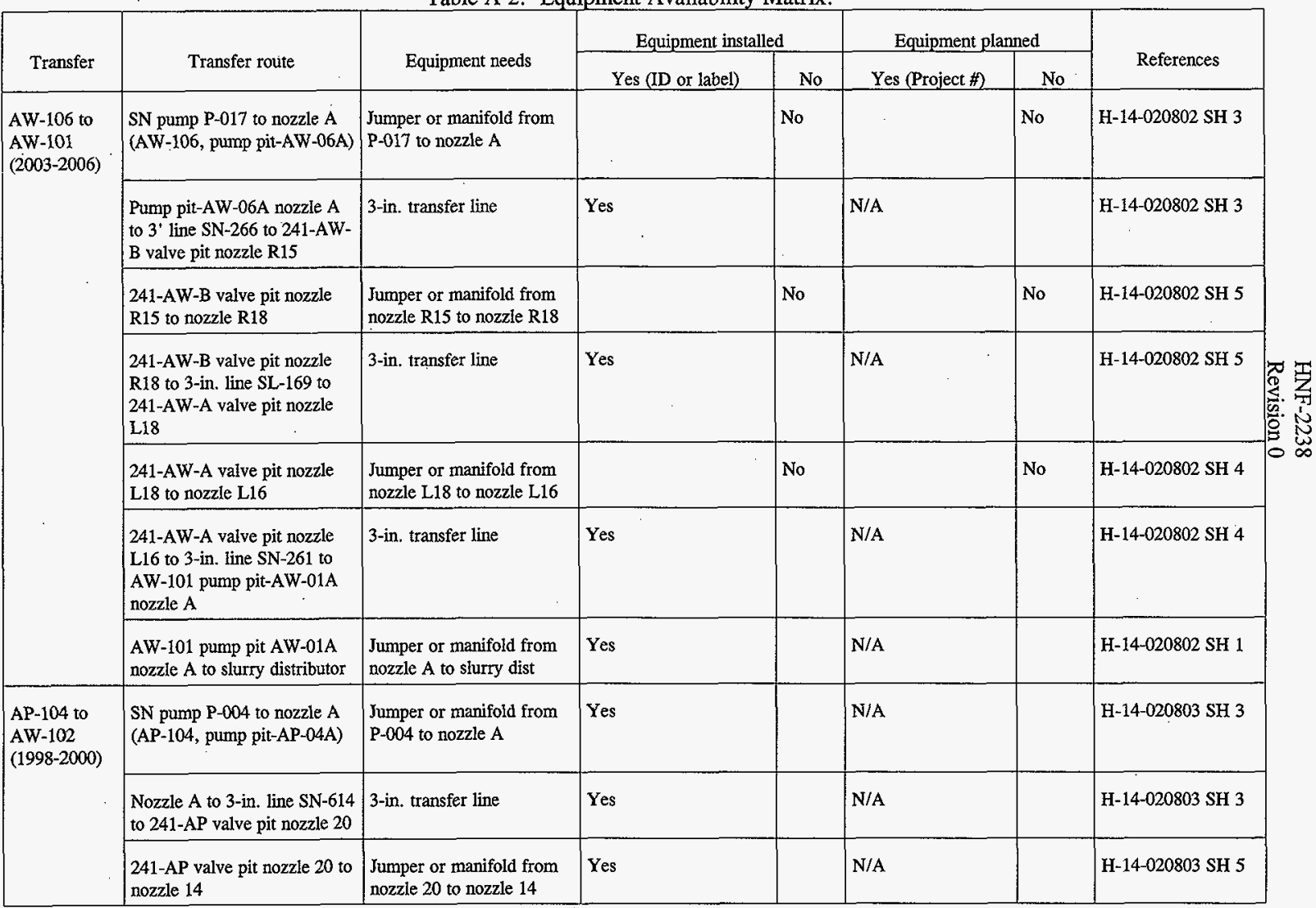


Table A-2. Equipment Availability Matrix.

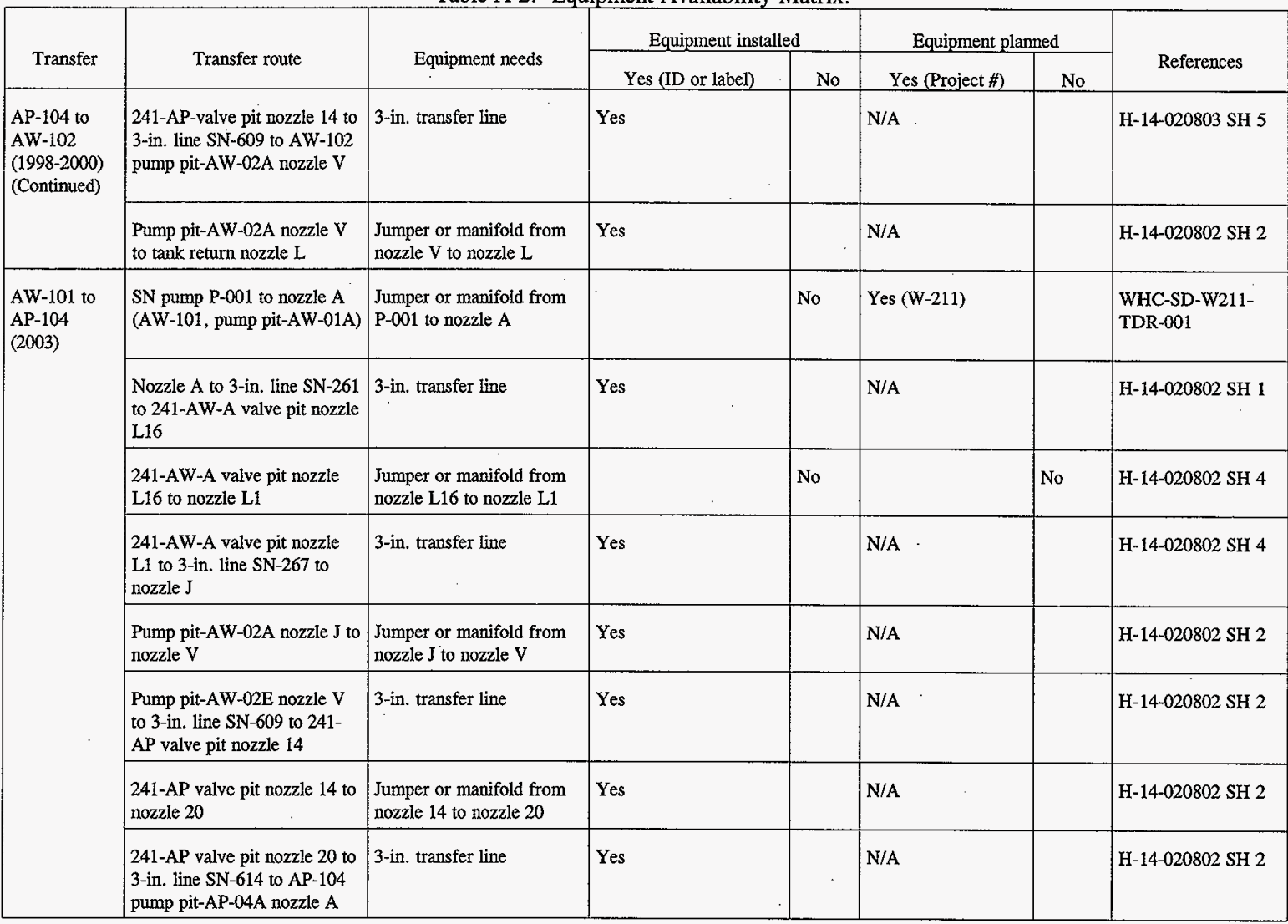


Table A-2. Equipment Availability Matrix.

\begin{tabular}{|c|c|c|c|c|c|c|c|}
\hline \multirow{2}{*}{ Transfer } & \multirow{2}{*}{ Transfer route } & \multirow{2}{*}{ Equipment needs } & \multicolumn{2}{|c|}{ Equipment installed } & \multicolumn{2}{|c|}{ Equipment planned } & \multirow{2}{*}{ References } \\
\hline & & & Yes (ID or label) & No & Yes (Project \#) & No & \\
\hline $\begin{array}{l}\text { AW-101 to } \\
\text { AP-104 } \\
\text { (2003) } \\
\text { (Continued) }\end{array}$ & $\begin{array}{l}\text { Pump pit-AP-04A nozzle } A \text { to } \\
\text { tank return nozzle } \mathrm{E}\end{array}$ & $\begin{array}{l}\text { Jumper or manifold from } \\
\text { nozzle } A \text { to nozzle } E\end{array}$ & Yes & & N/A & & H-14-020803 SH 3 \\
\hline \multirow{9}{*}{$\begin{array}{l}\text { AW-106 to } \\
\text { AN-101 } \\
(1999)\end{array}$} & $\begin{array}{l}\text { SN pump P-017 to nozzle A } \\
\text { (AW-106, pump pit-AW-06A) }\end{array}$ & $\begin{array}{l}\text { Jumper or manifold from } \\
\text { P-017 to nozzle A }\end{array}$ & & No & & No & H-14-020802 SH 3 \\
\hline & $\begin{array}{l}\text { Nozzle A to } 3 \text {-in. line SN-266 } \\
\text { to } 241-\mathrm{AW}-\mathrm{B} \text { valve pit nozzle } \\
\text { R15 }\end{array}$ & 3-in. transfer line & Yes & & N/A & & H-14-020802 SH 3 \\
\hline & $\begin{array}{l}\text { 241-AW-B valve pit nozzle } \\
\text { R15 to nozzle R19 }\end{array}$ & $\begin{array}{l}\text { Jumper or manifold from } \\
\text { nozzle R15 to nozzle R19 }\end{array}$ & . & No & & No & $\mathrm{H}-14-020802 \mathrm{SH} 5$ \\
\hline & $\begin{array}{l}\text { 241-AW-B pit nozzle R19 to } \\
\text { 3-in. line SN-271 to } 241-A W- \\
\text { A nozzle L19 }\end{array}$ & 3-in. transfer line & Yes & & N/A & & H-14-020802 SH 5 \\
\hline & $\begin{array}{l}\text { 241-AW-A pit nozzle L19 to } \\
\text { nozzle L2 }\end{array}$ & $\begin{array}{l}\text { Jumper or manifold from } \\
\text { nozzle } \mathrm{L} 19 \text { to nozzle } \mathrm{L} 2\end{array}$ & & No & & No & H-14-020802 SH 4 \\
\hline & $\begin{array}{l}\text { 241-AW-A pit nozzle L2 to } \\
\text { 3-in. line SN-220 to } 241-A-A \\
\text { pit nozzle L2 }\end{array}$ & 3-in. transfer line & Yes & & N/A & & H-14-020802 SH 4 \\
\hline & $\begin{array}{l}\text { 241-A-A pit nozzle L2 to } \\
\text { nozzle L1 }\end{array}$ & $\begin{array}{l}\text { Jumper or manifold from } \\
\text { nozzle L2 to nozzle L1 }\end{array}$ & No & & No & & Routing Board \\
\hline & $\begin{array}{l}\text { 241-A-A pit nozzle L1 to } \\
\text { 3-in. line SN-214/201 to } 241 \text { - } \\
\text { AX-A pit nozzle L1 }\end{array}$ & $\begin{array}{l}\text { 3-in. transfer line 3"SN- } \\
214 / 201\end{array}$ & Yes & & N/A & & SK-A-A, SK-AX-A \\
\hline & $\begin{array}{l}\text { 241-AX-A pit nozzle L1 to } \\
\text { nozzle L16 }\end{array}$ & $\begin{array}{l}\text { Jumper or manifold from } \\
\text { nozzle L1 to nozzle L16 }\end{array}$ & Yes & & N/A & & Routing Board \\
\hline
\end{tabular}


Table A-2. Equipment Availability Matrix.

\begin{tabular}{|c|c|c|c|c|c|c|c|}
\hline \multirow{2}{*}{ Transfer } & \multirow{2}{*}{ Transfer route } & \multirow{2}{*}{ Equipment needs } & \multicolumn{2}{|c|}{ Equipment installed } & \multicolumn{2}{|c|}{ Equipment planned } & \multirow{2}{*}{ References } \\
\hline & & & Yes (ID or label) & No & Yes (Project \#) & No & \\
\hline \multirow{6}{*}{$\begin{array}{l}\text { AW-106 to } \\
\text { AN-101 } \\
\text { (1999) } \\
\text { (Continued) }\end{array}$} & $\begin{array}{l}\text { 241-AX-A pit nozzle L16 to } \\
\text { 2-in. line SN-600 to sluice pit } \\
\text { 241-AZ-02B nozzle U5 }\end{array}$ & 2-in. transfer line & Yes & & N/A & & H-14-101080 \\
\hline & $\begin{array}{l}\text { 241-AZ-02B pit nozzle U5 to } \\
\text { nozzle U7 }\end{array}$ & $\begin{array}{l}\text { Jumper or manifold from } \\
\text { nozzle U5 to nozzle U7 }\end{array}$ & Yes & & $N / A$ & & Routing Board \\
\hline & $\begin{array}{l}\text { 241-AZ-02B pit nozzle } U 7 \text { to } \\
\text { 2-in. line SN-260 to valve pit- } \\
\text { 241-AN-B nozzle R2 }\end{array}$ & 2-in. transfer line & Yes & & N/A & & H-14-020801 SH 6 \\
\hline & $\begin{array}{l}\text { Valve Pit 241-AN-B } \\
\text { nozzle R2 to nozzle R15 }\end{array}$ & $\begin{array}{l}\text { Jumper or manifold from } \\
\text { nozzle R2 to nozzle R15 }\end{array}$ & Yes & & N/A & & Routing Board \\
\hline & $\begin{array}{l}\text { Valve Pit 241-AN-B nozzle } \\
\text { R2 to Tank 241-AN-101 } \\
\text { Central Pump Pit nozzle A }\end{array}$ & 3-in. transfer line & Yes & & N/A & & H-14-020801 SH 1 \\
\hline & $\begin{array}{l}\text { Tank 241-AN-101 Central } \\
\text { Pump Pit nozzle A to tank } \\
\text { return nozzle G }\end{array}$ & $\begin{array}{l}\text { Jumper or manifold from } \\
\text { nozzle A to nozzle G }\end{array}$ & Yes & & N/A & & H-14-020801 SH 1 \\
\hline \multirow{4}{*}{$\begin{array}{l}\text { AW-106 to } \\
\text { AN-106 } \\
(1999)\end{array}$} & $\begin{array}{l}\text { SN pump P-017 to nozzle A } \\
\text { (AW-106, pump pit-AW-06A) }\end{array}$ & $\begin{array}{l}\text { Jumper or manifold from } \\
\text { P-017 to nozzle A }\end{array}$ & & No & & No & H-14-020802 SH 3 \\
\hline & $\begin{array}{l}\text { Nozzle A to } 3 \text {-in. line SN-266 } \\
\text { to } 241 \text {-AW-B pit nozzle R15 }\end{array}$ & 3-in. transfer line & Yes & & N/A & & H-14-020802 SH 3 \\
\hline & $\begin{array}{l}\text { 241-AW-B pit nozzle R15 to } \\
\text { nozzle R19 }\end{array}$ & $\begin{array}{l}\text { Jumper or manifold from } \\
\text { nozzle R15 to nozzle R19 }\end{array}$ & & No & & No & H-14-020802 SH 5 \\
\hline & $\begin{array}{l}\text { 241-AW-B pit nozzle R19 to } \\
\text { 3-in. line SN-271 to 241-AW- } \\
\text { A pit nozzle L19 }\end{array}$ & 3-in. transfèt line & Ýs & & NTA & & H-14-020802 SH 5 \\
\hline
\end{tabular}


Table A-2. Equipment Availability Matrix.

\begin{tabular}{|c|c|c|c|c|c|c|c|}
\hline \multirow{2}{*}{ Transfer } & \multirow{2}{*}{ Transfer route } & \multirow{2}{*}{ Equipment needs } & \multicolumn{2}{|c|}{ Equipment installed } & \multicolumn{2}{|c|}{ Equipment planned } & \multirow{2}{*}{ References } \\
\hline & & & Yes (ID or label) & No & Yes (Project \#) & No & \\
\hline \multirow{10}{*}{$\begin{array}{l}\text { AW-106 to } \\
\text { AN-106 } \\
\text { (1999) } \\
\text { (Continued) }\end{array}$} & $\begin{array}{l}\text { 241-AW-A pit nozzle L19 to } \\
\text { nozzle L2 }\end{array}$ & $\begin{array}{l}\text { Jumper or manifold from } \\
\text { nozzle L19 to nozzle L2 }\end{array}$ & & No & & No & H-14-020802 SH 4 \\
\hline & $\begin{array}{l}\text { 241-AW-A pit nozzle } \mathrm{L} 2 \text { to } \\
\text { 3-in. line SN-220 to } 241-\mathrm{A}-\mathrm{A} \\
\text { pit nozzle } \mathrm{L} 2\end{array}$ & 3-in. transfer line & Yes & & N/A & & H-14-020802 SH 4 \\
\hline & $\begin{array}{l}\text { 241-A-A pit nozzle L2 to } \\
\text { nozzle L1 }\end{array}$ & $\begin{array}{l}\text { Jumper or manifold from } \\
\text { nozzle L2 to nozzle L1 }\end{array}$ & & No & . & No & Routing Board \\
\hline & $\begin{array}{l}\text { 241-A-A pit nozzle L1 to } \\
\text { 3-in. line SN-214/201 to } 241 \text { - } \\
\text { AX-A nozzle L1 }\end{array}$ & 3-in. transfer line & Yes & . & N/A & & H-14-101080 \\
\hline & $\begin{array}{l}\text { 241-AX-A pit nozzle L1 to } \\
\text { nozzle L16 }\end{array}$ & $\begin{array}{l}\text { Jumper or manifold from } \\
\text { nozzle L1 to nozzle L16 }\end{array}$ & Yes & & N/A & & Routing Board \\
\hline & $\begin{array}{l}\text { 241-AX-A pit nozzle L16 to } \\
\text { 2-in. line SN-600 to } 241-A Z \text { - } \\
102 \text { 02B pit nozzle U5 }\end{array}$ & 2-in. transfer line & Yes & & N/A & & H-14-101080 \\
\hline & $\begin{array}{l}\text { 241-AZ-102 02B pit nozzle } \\
\text { U5 to nozzle U7 }\end{array}$ & $\begin{array}{l}\text { Jumper or manifold from } \\
\text { nozzle U5 to nozzle U7 }\end{array}$ & Yes & & N/A & & Routing Board \\
\hline & $\begin{array}{l}\text { 241-AZ-102 02B pit nozzle } \\
\text { U7 to 2-in. line SN-260 to } \\
\text { valve pit } 241 \text {-AN-B nozzle R2 }\end{array}$ & 2-in. transfer line & Yes & & N/A & & H-14-020801 SH 6 \\
\hline & $\begin{array}{l}\text { Valve pit } 241-A N-B \text { nozzle } \\
\text { R2 to nozzle R19 }\end{array}$ & $\begin{array}{l}\text { Jumper or manifold from } \\
\text { nozzle R2 to nozzle R19 }\end{array}$ & Yes & & N/A & & Routing Board \\
\hline & $\begin{array}{l}241-A N-B \text { nozzle R19 to } 3 \text {-in. } \\
\text { line SN-268 to Valve pit } 241- \\
\text { AN-A nozzle L19 }\end{array}$ & 3-in. transfer line & Yes & & N/A & & H-14-020801 SH 6 \\
\hline
\end{tabular}




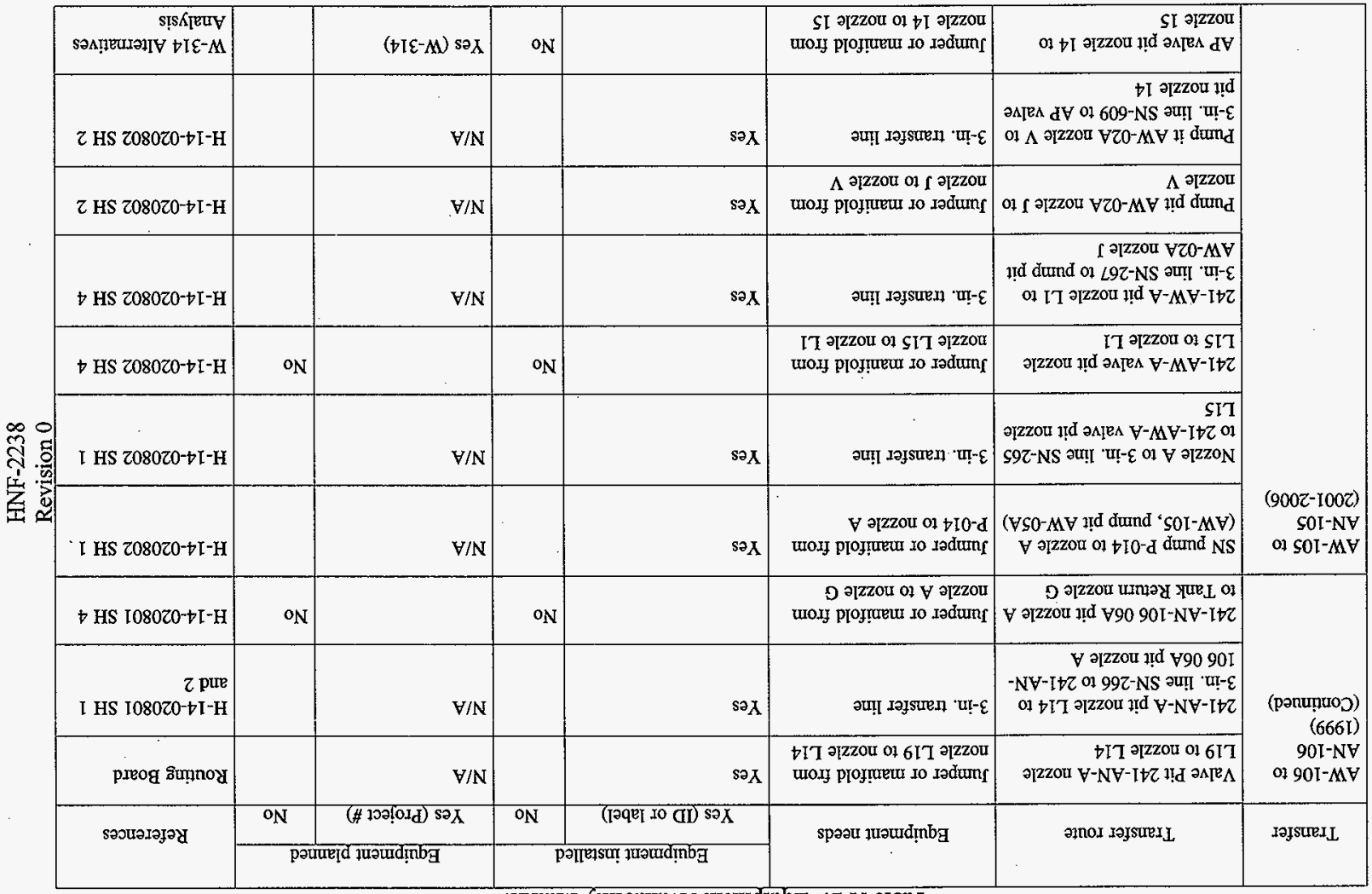


Table A-2. Equipment Availability Matrix.

\begin{tabular}{|c|c|c|c|c|c|c|c|}
\hline \multirow{2}{*}{ Transfer } & \multirow{2}{*}{ Transfer route } & \multirow{2}{*}{ Equipment needs } & \multicolumn{2}{|c|}{ Equipment installed } & \multicolumn{2}{|c|}{ Equipment planned } & \multirow{2}{*}{ References } \\
\hline & & & Yes (ID or label) & No & Yes (Project \#) & No & \\
\hline \multirow{9}{*}{$\begin{array}{l}\text { AW-105 to } \\
\text { AN-105 } \\
\text { (2001-2006) } \\
\text { (continued) }\end{array}$} & $\begin{array}{l}\text { 241-AP Valve pit nozzle } 15 \text { to } \\
\text { 3-in. line NVP/APVP to } \\
\text { valve pit NVP nozzle E }\end{array}$ & 3-in. transfer line & & No & Yes (W-314) & & $\begin{array}{l}\text { W-314 Alternatives } \\
\text { Analysis }\end{array}$ \\
\hline & $\begin{array}{l}\text { Valve pit NVP nozzle E to } \\
\text { nozzle A }\end{array}$ & $\begin{array}{l}\text { Jumper or manifold from } \\
\text { nozzle } \mathrm{E} \text { to nozzle } \mathrm{A}\end{array}$ & & No & Yes (W-314) & & $\begin{array}{l}\text { W-314 Alternatives } \\
\text { Analysis }\end{array}$ \\
\hline & $\begin{array}{l}\text { NVP valve pit nozzle A to } \\
\text { 3-in. line AN101A/NVP to } \\
\text { pump pit AN-01A nozzle D }\end{array}$ & 3-in. transfer line & & No & Yes $(W-314)$ & & $\begin{array}{l}\text { W-314 Alternatives } \\
\text { Analysis }\end{array}$ \\
\hline & $\begin{array}{l}\text { Pump pit AN-01A nozzle D } \\
\text { to nozzle } \mathrm{A}\end{array}$ & $\begin{array}{l}\text { Jumper or manifold from } \\
\text { nozzle D to nozzle A }\end{array}$ & & No & Yes (W-314) & & $\begin{array}{l}\text { W-314 Alternatives } \\
\text { Analysis }\end{array}$ \\
\hline & $\begin{array}{l}\text { Pump pit AN-01A nozzle A } \\
\text { to 3-in. line SN-261 to } 241- \\
\text { AN-B valve pit nozzle R15 }\end{array}$ & 3-in. transfer line & Yes & & N/A & & H-14-020801 SH1 \\
\hline & $\begin{array}{l}\text { 241-AN-B valve pit nozzle } \\
\text { R15 to nozzle R19 }\end{array}$ & $\begin{array}{l}\text { Jumper manifold from } \\
\text { nozzle R15 to nozzle R19 }\end{array}$ & Yes & & N/A & & H-14-020801 SH6 \\
\hline & $\begin{array}{l}\text { 241-AN-B valve pit nozzle } \\
\text { R19 to 3-in. line SN-268 to } \\
\text { 241-AN-A valve pit nozzle } \\
\text { L19 }\end{array}$ & 3-in. transfer line & Yes & & N/A & & H-14-020801 SH6 \\
\hline & $\begin{array}{l}\text { 241-AN-A valve pit nozzle } \\
\text { L19 to nozzle L16 }\end{array}$ & $\begin{array}{l}\text { Jumper manifold from } \\
\text { nozzle L19 to nozzle L16 }\end{array}$ & & No & Yes (W-314) & & $\begin{array}{l}\text { W-314 Alternatives } \\
\text { Analysis }\end{array}$ \\
\hline & $\begin{array}{l}\text { 241-AN-A valve pit nozzle } \\
\text { L16 to 3-in. line SN-265 to } \\
\text { pump pit AN-05A nozzle A }\end{array}$ & 3-in. transfer line & Yes & & N/A & & H-14-020801 SH5 \\
\hline
\end{tabular}


Table A-2. Equipment Availability Matrix.

\begin{tabular}{|c|c|c|c|c|c|c|c|}
\hline \multirow{2}{*}{ Transfer } & \multirow{2}{*}{ Transfer route } & \multirow{2}{*}{ Equipment needs } & \multicolumn{2}{|c|}{ Equipment installed } & \multicolumn{2}{|c|}{ Equipment planned } & \multirow{2}{*}{ References } \\
\hline & & & Yes (ID or label) & No & Yes (Project \#) & No & \\
\hline $\begin{array}{l}\text { AW-105 to } \\
\text { AN-105 } \\
\text { (2001-2006) } \\
\text { (continued) }\end{array}$ & $\begin{array}{l}\text { Pump pit AN-05A nozzle A } \\
\text { to tank return nozzle } G\end{array}$ & $\begin{array}{l}\text { Jumper manifold from } \\
\text { nozzle } A \text { to nozzle } G\end{array}$ & & No & Yes (W-211) & & WHC-SD-TDR-001 \\
\hline \multirow{5}{*}{$\begin{array}{l}\text { AW-105 to } \\
\text { AW-102 } \\
(2000)\end{array}$} & $\begin{array}{l}\text { SN pump P-014 to nozzle A } \\
\text { (AW-105, pump pit-AW-05A) }\end{array}$ & $\begin{array}{l}\text { Jumper or manifold from } \\
\text { P-014 to nozzle A }\end{array}$ & Yes & & N/A & & H-14-020802 SH 1 \\
\hline & $\begin{array}{l}\text { Nozzle A to 3-in, line SN-265 } \\
\text { to } 241 \text {-AW-A pit nozzle L15 }\end{array}$ & 3-in. transfer line & Yes & & N/A & & H-14-020802 SH 1 \\
\hline & $\begin{array}{l}\text { 241-AW-A pit nozzle } \mathrm{L} 15 \text { to } \\
\text { nozzle L1 }\end{array}$ & $\begin{array}{l}\text { Jumper or manifold from } \\
\text { nozzle L15 to nozzle L1 }\end{array}$ & & No & & No & H-14-020802 SH 4 \\
\hline & $\begin{array}{l}\text { 241-AW-A pit nozzle L1 to } \\
\text { 3-in. line SN-267 to AW-102 } \\
\text { pump pit-AW-02A nozzle J }\end{array}$ & 3-in. transfer line & Yes & & N/A & & H-14-020802 SH 4 \\
\hline & $\begin{array}{l}\text { Pump pit-AW-02A nozzle } \mathrm{J} \text { to } \\
\text { tank return nozzle } \mathbf{L}\end{array}$ & $\begin{array}{l}\text { Jumper or manifold from } \\
\text { nozzle } \mathrm{J} \text { to nozzle } \mathrm{L}\end{array}$ & Yes & & N/A & & H-14-020802 SH 2 \\
\hline \multirow{3}{*}{$\begin{array}{l}S Y-102 \text { to } \\
\text { AW-102 } \\
\text { (supernate, } \\
2002 \text { ) }\end{array}$} & $\begin{array}{l}\text { (From Cross-Site Tie-In Point } \\
\text { [XTIP] west of 244-A Lift } \\
\text { Station) }\end{array}$ & & & & & & \\
\hline & $\begin{array}{l}\text { XTIP to 3-in. line XTIP/AN- } \\
101 \text { to pump pit AN-01A } \\
\text { nozzle C }\end{array}$ & 3-in. transfer line & & No & Yes (W-314) & & $\begin{array}{l}\text { W-314 Alternatives } \\
\text { Analysis }\end{array}$ \\
\hline & $\begin{array}{l}\text { Pump pit AN-01A nozzle } C \\
\text { to nozzle } D\end{array}$ & $\begin{array}{l}\text { Jumper or manifold from } \\
\text { nozzle } C \text { to nozzle } D\end{array}$ & & No & Yes (W-314) & & $\begin{array}{l}\text { W-314 Alternatives } \\
\text { Analysis }\end{array}$ \\
\hline
\end{tabular}


Table A-2. Equipment Availability Matrix.

\begin{tabular}{|c|c|c|c|c|c|c|c|}
\hline Transfer & Transfer route & Equipment needs & \multicolumn{2}{|c|}{ Equipment installed } & \multicolumn{2}{|c|}{ Equipment planned } & References \\
\hline \multirow{4}{*}{$\begin{array}{l}\text { SY-102 to } \\
\text { AW-102 } \\
\text { (supernate, } \\
2002 \text { ) } \\
\text { (Continued) }\end{array}$} & $\begin{array}{l}\text { Pump pit AN-01A nozzle D } \\
\text { to } 3 \text {-in. line AN101A/NVP to } \\
\text { valve pit NVP nozzle A }\end{array}$ & 3-in. transfer line & & No & Yes (W-314). & & $\begin{array}{l}\text { W-314 Alternatives } \\
\text { Analysis }\end{array}$ \\
\hline & $\begin{array}{l}\text { Valve Pit NVP nozzle A to } \\
\text { nozzle E }\end{array}$ & $\begin{array}{l}\text { Jumper or manifold from } \\
\text { nozzle B to nozzle } \mathrm{E}\end{array}$ & & No & Yes (W-314) & & $\begin{array}{l}\text { W-314 Alternatives } \\
\text { Analysis }\end{array}$ \\
\hline & $\begin{array}{l}\text { Valve Pit NVP nozzie E to } 3 \text { - } \\
\text { in. line NVP/APVP to } 241- \\
\text { AP valve pit nozzle } 15\end{array}$ & 3-in. transfer line & $\therefore$ & No & Yes (W-314) & & $\begin{array}{l}\text { W-314 Alternatives } \\
\text { Analysis }\end{array}$ \\
\hline & $\begin{array}{l}\text { 241-AP valve pit nozzle } 15 \text { to } \\
\text { nozzle } 14\end{array}$ & $\begin{array}{l}\text { Jumper or manifold from } \\
\text { nozzle } 15 \text { to } 14\end{array}$ & & No & Yes (W-314) & & $\begin{array}{l}\text { W-314 Alternatives } \\
\text { Analysis }\end{array}$ \\
\hline \multirow[t]{3}{*}{$\begin{array}{l}\text { SY-102 to } \\
\text { AW-105 } \\
\text { (supernate, } \\
2005 \text { ) }\end{array}$} & $\begin{array}{l}\text { (From Cross-Site Tie-In Point } \\
\text { [XTIP] west of } 244-\text { A Lift } \\
\text { Station) }\end{array}$ & & & & & & \\
\hline & $\begin{array}{l}\text { XTIP to 3-in. line XTIP/AN- } \\
101 \text { to pump pit AN-01A } \\
\text { nozzle C }\end{array}$ & 3-in. transfer line & & No & Yes (W-314) & & $\begin{array}{l}\text { W-314 Alternatives } \\
\text { Analysis }\end{array}$ \\
\hline & $\begin{array}{l}\text { Pump pit AN-01A nozzle C } \\
\text { to nozzle D }\end{array}$ & $\begin{array}{l}\text { Jumper or manifold from } \\
\text { nozzle } C \text { to nozzle } D\end{array}$ & & No & Yes (W-314) & & $\begin{array}{l}\text { W-314 Alternatives } \\
\text { Analysis }\end{array}$ \\
\hline
\end{tabular}


Table A-2. Equipment Availability Matrix.

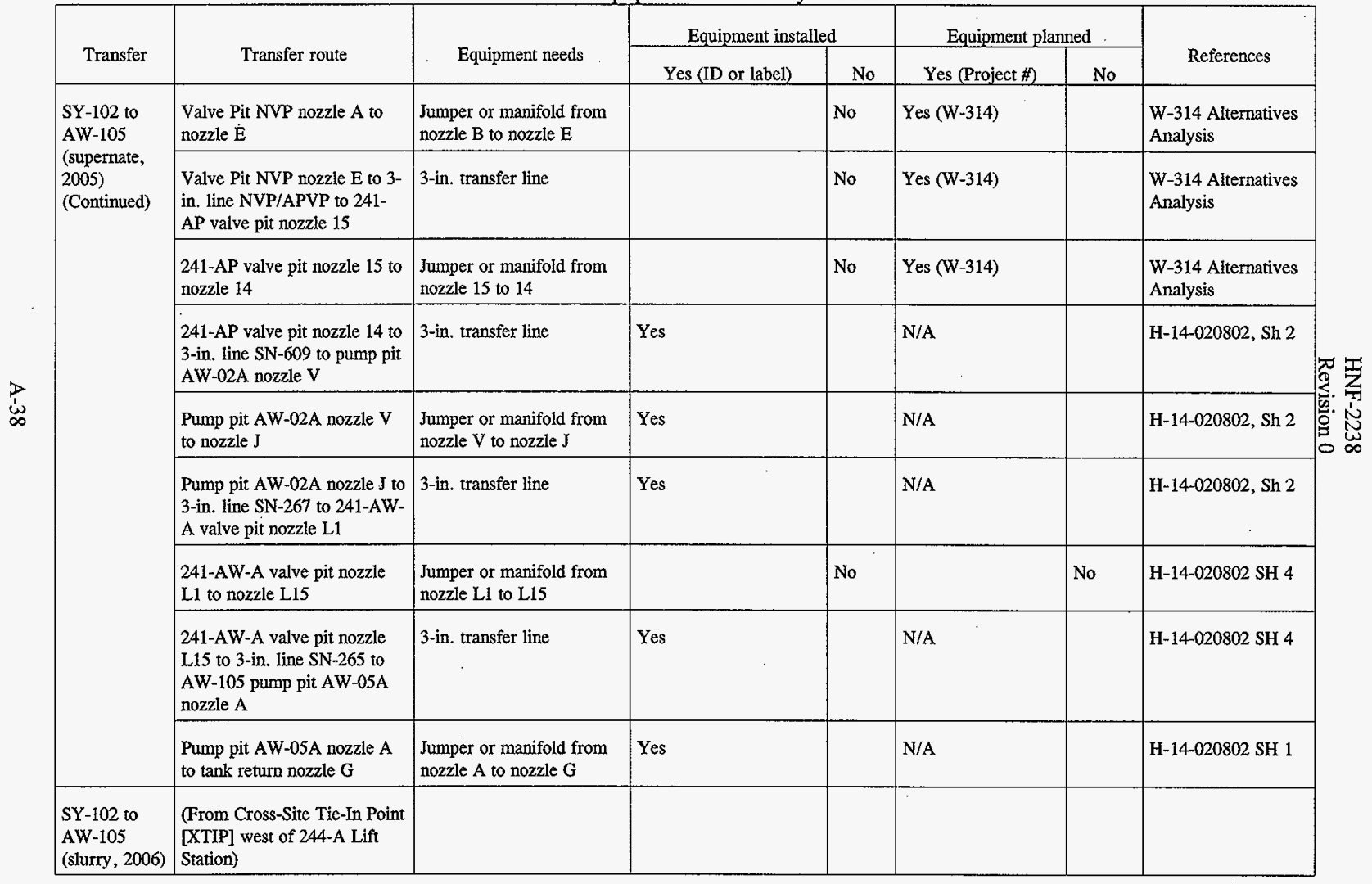


Table A-2. Equipment Availability Matrix.

\begin{tabular}{|c|c|c|c|c|c|c|c|}
\hline \multirow{2}{*}{ Transfer } & \multirow{2}{*}{ Transfer route } & \multirow{2}{*}{ Equipment needs } & \multicolumn{2}{|c|}{ Equipment installed } & \multicolumn{2}{|c|}{ Equipment planned } & \multirow{2}{*}{ References } \\
\hline & & & Yes (ID or label) & No & Yes (Project \#) & No & \\
\hline \multirow{9}{*}{$\begin{array}{l}\text { SY-102 to } \\
\text { AW-105 } \\
\text { (siurry, 2006) } \\
\text { (Continued) }\end{array}$} & $\begin{array}{l}\text { XTIP to High-Pressure 3-in. } \\
\text { line XTIP/AN-104 to Tank } \\
\text { 241-AN-104 riser TBD }\end{array}$ & 3-in. transfer line & & No & Yes (W-314) & & $\begin{array}{l}\text { W-314 Alternatives } \\
\text { Analysis }\end{array}$ \\
\hline & $\begin{array}{l}\text { Pump pit AN-04A, Slurry } \\
\text { Pump P-TBD to nozzle A }\end{array}$ & $\begin{array}{l}\text { Pump and Jumper or } \\
\text { manifold from P-TBD to } \\
\text { nozzle A }\end{array}$ & & No & Yes (W-314) & & $\begin{array}{l}\text { W-314 Alternatives } \\
\text { Analysis }\end{array}$ \\
\hline & $\begin{array}{l}\text { Pump pit AN-04A nozzle A } \\
\text { to 3-in. line SN-264 to vaive } \\
\text { pit AN-A nozzle L15 }\end{array}$ & 3-in. transfer line & Yes & & N/A & & H-14-020802 SH 3 \\
\hline & $\begin{array}{l}\text { 241-AN-A valve pit nozzle } \\
\text { L15 to nozzle L19 }\end{array}$ & $\begin{array}{l}\text { Jumper or manifold from } \\
\text { nozzle L15 to nozzle L19 }\end{array}$ & & No & Yes (W-314) & & $\begin{array}{l}\text { W-314 Alternatives } \\
\text { Analysis }\end{array}$ \\
\hline & $\begin{array}{l}\text { 241-AN-A valve pit nozzle } \\
\text { L19 to } 3 \text {-in. line SN-268 to } \\
\text { 241-AN-B valve pit nozzle } \\
\text { R19 }\end{array}$ & 3-in. transfer line & Yes & & N/A & & H-14-020802 SH 5 \\
\hline & $\begin{array}{l}\text { 241-AN-B valve pit nozzle } \\
\text { R19 to nozzle R15 }\end{array}$ & $\begin{array}{l}\text { Jumper or manifold from } \\
\text { nozzle R14 to nozzle R15 }\end{array}$ & Yes & & N/A & & H-14-020802 SH 6 \\
\hline & $\begin{array}{l}\text { 241-AN-B valve pit nozzle } \\
\text { R15 to 3-in. line SN-261 to } \\
\text { pump pit AN-01A nozzle A }\end{array}$ & 3-in. transfer line & Yes & & N/A & & H-14-020802 SH 6 \\
\hline & $\begin{array}{l}\text { Pump pit AN-01A nozzle } A \\
\text { to nozzle } D\end{array}$ & $\begin{array}{l}\text { Jumper or manifold from } \\
\text { nozzle } A \text { to nozzle } D\end{array}$ & & No & Yes (W-314) & & $\begin{array}{l}\text { W-314 Alternatives } \\
\text { Analysis }\end{array}$ \\
\hline & $\begin{array}{l}\text { Pump pit AN-01A nozzle D } \\
\text { to } 3 \text {-in. line AN101A/NVP to } \\
\text { valve pit NVP nozzle A }\end{array}$ & 3-in. transfer line & & No & Yes (W-314) & & $\begin{array}{l}\text { W-314 Alternatives } \\
\text { Analysis }\end{array}$ \\
\hline
\end{tabular}


Table A-2. Equipment Availability Matrix.

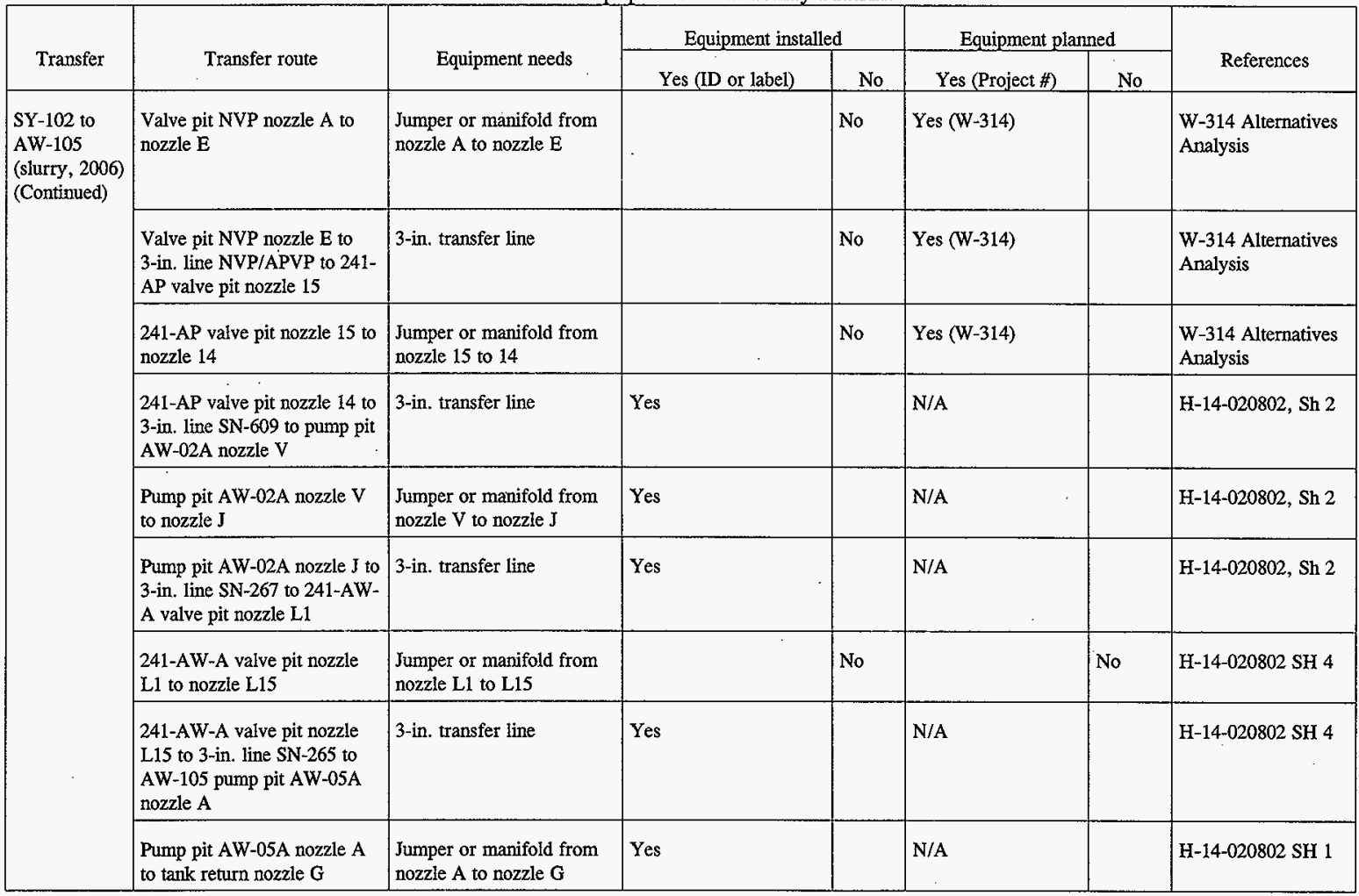


Table A-3. Piping Parameters.

\begin{tabular}{|c|c|c|c|c|c|c|}
\hline $\begin{array}{c}\text { Line number } \\
\text { (connection points) }\end{array}$ & $\begin{array}{c}\text { Material of } \\
\text { construction } \\
\text { (material code) }\end{array}$ & $\begin{array}{l}\text { Maximum operating } \\
\text { pressure }\end{array}$ & $\begin{array}{c}\text { Maximum } \\
\text { operating } \\
\text { temperature }\end{array}$ & Test pressure & $\begin{array}{l}\text { Flanged/ } \\
\text { butt-welded }\end{array}$ & $\begin{array}{c}\text { Reference: } \\
\text { construction spec } \\
\text { \# and drawing \# }\end{array}$ \\
\hline $\begin{array}{c}\text { 3"LIQW-702 } \\
\text { (204-AR to 241-A-A) }\end{array}$ & $\begin{array}{l}\text { Stainless Steel } \\
\text { (M8) }\end{array}$ & $0.93 \mathrm{MPa}(120 \mathrm{psig})$ & $38^{\circ} \mathrm{C}\left(100^{\circ} \mathrm{F}\right)$ & 1.3 $\mathrm{MPa}$ (180 psig) & butt-welded & $\begin{array}{l}\text { B-133-C1 } \\
\text { H-2-70706 }\end{array}$ \\
\hline $\begin{array}{c}\text { 2"SL-166 } \\
\text { (241-AW-B to 241-AW-106) }\end{array}$ & $\begin{array}{l}\text { Carbon Steel } \\
\text { (M25) }\end{array}$ & $2.9 \mathrm{MPa}$ (400 psig) & $171^{\circ} \mathrm{C}\left(340^{\circ} \mathrm{F}\right)$ & 4.2 $\mathrm{MPa}$ (600 psig) & butt-welded & $\begin{array}{l}\text { B-120-C7 } \\
\text { H-14-020802 }\end{array}$ \\
\hline $\begin{array}{c}2 " S L-167 \\
\text { (242-A to 241-AW-B) }\end{array}$ & $\begin{array}{l}\text { Carbon Steel } \\
\text { (M25) }\end{array}$ & $2.9 \mathrm{MPa}(400 \mathrm{psig})$ & $171^{\circ} \mathrm{C}\left(340^{\circ} \mathrm{F}\right)$ & $4.2 \mathrm{MPa}$ (600 psig) & butt-welded & $\begin{array}{c}\text { B-120-C7 } \\
\mathrm{H}-14-020802\end{array}$ \\
\hline $\begin{array}{c}3 " \mathrm{SN}-220 \\
\text { (241-A-A to } 241-\mathrm{AW}-\mathrm{A}) \\
\end{array}$ & $\begin{array}{l}\text { Carbon Steel } \\
\text { (M25) } \\
\end{array}$ & $1.7 \mathrm{MPa}(230 \mathrm{psig})$ & $166^{\circ} \mathrm{C}\left(330^{\circ} \mathrm{F}\right)$ & 2.5 $\mathrm{MPa}(350 \mathrm{psig})$ & butt-welded & $\begin{array}{c}\text { B-102-C1 } \\
\text { H-14-020802 }\end{array}$ \\
\hline $\begin{array}{l}3^{n \text { SNN-261 }} \\
\text { (241-AW-A to 241-AW-101) }\end{array}$ & $\begin{array}{l}\text { Carbon Steel } \\
\text { (M25) }\end{array}$ & $2.0 \mathrm{MPa}(275 \mathrm{psig})$ & $171^{\circ} \mathrm{C}\left(340^{\circ} \mathrm{F}\right)$ & 3.2 MPa (450 psig) & butt-welded & $\begin{array}{c}\text { B-120-C7 } \\
\mathrm{H}-14-020802 \\
\end{array}$ \\
\hline $\begin{array}{c}3 " \text { SN-262 } \\
\text { (241-AW-B to 241-AW-102) }\end{array}$ & $\begin{array}{l}\text { Carbon Steel } \\
\text { (M25) }\end{array}$ & $2.0 \mathrm{MPa}(275 \mathrm{psig})$ & $171^{\circ} \mathrm{C}\left(340^{\circ} \mathrm{F}\right)$ & $3.2 \mathrm{MPa}(450 \mathrm{psig})$ & butt-welded & $\begin{array}{c}\text { B-120-C7 } \\
\mathrm{H}-14-020802 \\
\end{array}$ \\
\hline $\begin{array}{c}3^{\prime \prime} \mathrm{SN}-263 \\
\text { (241-AW-A to } 241-\mathrm{AW}-103)\end{array}$ & $\begin{array}{l}\text { Carbon Steel } \\
\text { (M25) }\end{array}$ & $2.0 \mathrm{MPa}(275 \mathrm{psig})$ & $171^{\circ} \mathrm{C}\left(340^{\circ} \mathrm{F}\right)$ & 3.2 $\mathrm{MPa}(450 \mathrm{psig})$ & butt-welded & $\begin{array}{l}\text { B-120-C7 } \\
\mathrm{H}-14-020802\end{array}$ \\
\hline $\begin{array}{c}\text { 3"SN-264 } \\
\text { (241-AW-B to 241-AW-104) }\end{array}$ & $\begin{array}{l}\text { Carbon Steel } \\
\text { (M25) }\end{array}$ & $2.0 \mathrm{MPa}(275 \mathrm{psig})$ & $171^{\circ} \mathrm{C}\left(340^{\circ} \mathrm{F}\right)$ & 3.2 MPa (450 psig) & butt-welđed & $\begin{array}{c}\text { B-120-C7 } \\
\text { H-14-020802 }\end{array}$ \\
\hline $\begin{array}{c}3 " \text { SN-265 } \\
\text { (241-AW-A to 241-AW-105) }\end{array}$ & $\begin{array}{l}\text { Carbon Steel } \\
\text { (M25) }\end{array}$ & $2.0 \mathrm{MPa}$ (275 psig) & $171^{\circ} \mathrm{C}\left(340^{\circ} \mathrm{F}\right)$ & $3.2 \mathrm{MPa}(450 \mathrm{psig})$ & butt-welded & $\begin{array}{c}\text { B-120-C7 } \\
\text { H-14-020802 }\end{array}$ \\
\hline $\begin{array}{c}3 " \text { SN-266 } \\
\text { (241-AW-B to 241-AW-106) }\end{array}$ & $\begin{array}{l}\text { Carbon Steel } \\
\quad \text { (M25) }\end{array}$ & $2.0 \mathrm{MPa}$ (275 psig) & $171^{\circ} \mathrm{C}\left(340^{\circ} \mathbf{F}\right)$ & 3.2 $\mathrm{MPa}$ (450 psig) & butt-welded & $\begin{array}{c}\mathrm{B}-120-\mathrm{C} 7 \\
\mathrm{H}-14-020802\end{array}$ \\
\hline $\begin{array}{c}3 \text { "SN-267 } \\
\text { (241-AW-A to 241-AW-102) }\end{array}$ & $\begin{array}{l}\text { Carbon Steel } \\
\text { (M25) }\end{array}$ & $2.0 \mathrm{MPa}$ (275 psig) & $171^{\circ} \mathrm{C}\left(340^{\circ} \mathbf{F}\right)$ & 3.2 $\mathrm{MPa}$ (450 psig) & butt-welded & $\begin{array}{c}\mathrm{B}-120-\mathrm{C} 7 \\
\mathrm{H}-14-020802\end{array}$ \\
\hline $\begin{array}{c}\text { 3"SN-268 } \\
\text { (241-AW-B to 241-AW-102) } \\
\end{array}$ & $\begin{array}{l}\text { Carbon Steel } \\
\text { (M25) }\end{array}$ & $2.0 \mathrm{MPa}$ (275 psig) & $171^{\circ} \mathrm{C}\left(340^{\circ} \mathrm{F}\right)$ & 3.2 $\mathrm{MPa}$ (450 psig) & butt-welded & $\begin{array}{c}\text { B-120-C7 } \\
\text { H-14-020802 }\end{array}$ \\
\hline $\begin{array}{c}3^{n " S N-269} \\
\text { (241-AW-102 to 242-A) }\end{array}$ & $\begin{array}{l}\text { Carbon Steel } \\
\text { (M25) }\end{array}$ & $2.0 \mathrm{MPa} \cdot(275 \mathrm{psig})$ & $171^{\circ} \mathrm{C}\left(340^{\circ} \mathrm{F}\right)$ & 3.2 $\mathrm{MPa}(450 \mathrm{psig})$ & butt-welded & $\begin{array}{c}\text { B-120-C7 } \\
\mathrm{H}-14-020802\end{array}$ \\
\hline
\end{tabular}


Table A-3. Piping Parameters.

\begin{tabular}{|c|c|c|c|c|c|c|}
\hline $\begin{array}{c}\text { Line number } \\
\text { (connection points) }\end{array}$ & $\begin{array}{c}\text { Material of } \\
\text { construction } \\
\text { (material code) }\end{array}$ & $\begin{array}{l}\text { Maximum operating } \\
\text { pressure }\end{array}$ & $\begin{array}{l}\text { Maximum } \\
\text { operating } \\
\text { temperature }\end{array}$ & Test pressure & $\begin{array}{l}\text { Flanged/ } \\
\text { butt-welded }\end{array}$ & $\begin{array}{c}\text { Reference: } \\
\text { construction spec } \\
\text { \# and drawing \# }\end{array}$ \\
\hline $\begin{array}{c}\text { 3"SN-270 } \\
\text { (242-A to 241-AW-102) }\end{array}$ & $\begin{array}{l}\text { Carbon Steel } \\
\text { (M25) }\end{array}$ & $2.0 \mathrm{MPa}(275 \mathrm{psig})$ & $171^{\circ} \mathrm{C}\left(340^{\circ} \mathrm{F}\right)$ & $3.2 \mathrm{MPa}$ (450 psig) & butt-welded & $\begin{array}{c}\text { B-120-C7 } \\
\text { H-14-020802 }\end{array}$ \\
\hline $\begin{array}{c}3 \text { "SN-271 } \\
\text { (241-AW-A to 241-AW-B) }\end{array}$ & $\begin{array}{l}\text { Carbon Steel } \\
\text { (M25) }\end{array}$ & $2.0 \mathrm{MPa}(275 \mathrm{psig})$ & $171^{\circ} \mathrm{C}\left(340^{\circ} \mathrm{F}\right)$ & $3.2 \mathrm{MPa}(450 \mathrm{psig})$ & butt-weided & $\begin{array}{c}\text { B-120-C7 } \\
\text { H-14-020802 }\end{array}$ \\
\hline $\begin{array}{c}3^{\text {"SN-272 }} \\
\text { (241-AW-102 Pit 02E to } \\
\text { 241-AW-102 pit 02A) }\end{array}$ & $\begin{array}{l}\text { Carbon Steel } \\
\text { (M25) }\end{array}$ & $2.0 \mathrm{MPa}(275 \mathrm{psig})$ & $171^{\circ} \mathrm{C}\left(340^{\circ} \mathrm{F}\right)$ & 3.2 $\mathrm{MPa}(450 \mathrm{psig})$ & butt-welded & $\begin{array}{c}\text { B-120-C7 } \\
\mathrm{H}-14-020802\end{array}$ \\
\hline $\begin{array}{c}\text { 3"SN-609 } \\
\text { (241-AW-102 to 241-AP) }\end{array}$ & $\begin{array}{l}\text { Carbon Steel } \\
\text { (M25) }\end{array}$ & $2.9 \mathrm{MPa}$ (400 psig) & $171^{\circ} \mathrm{C}\left(340^{\circ} \mathrm{F}\right)$ & 4.2 $\mathrm{MPa}(600 \mathrm{psig})$ & butt-welded & $\begin{array}{c}\text { B-340-C7 } \\
\text { H-14-020803 }\end{array}$ \\
\hline $\begin{array}{c}3 " \text { SN-610 } \\
\text { (241-AW-102 to 241-AP) }\end{array}$ & $\begin{array}{l}\text { Carbon Steel } \\
\text { (M25) }\end{array}$ & $2.9 \mathrm{MPa}(400 \mathrm{psig})$ & $171^{\circ} \mathrm{C}\left(340^{\circ} \mathrm{F}\right)$ & $4.2 \mathrm{MPa}(600 \mathrm{psig})$ & butt-welded & $\begin{array}{c}\text { B-340-C7 } \\
\text { H-14-020803. }\end{array}$ \\
\hline
\end{tabular}


HNF-2238

Revision 0

\section{APPENDIX B}

\section{TRANSFER ISSUES}


HNF-2238

Revision 0

This page intentionally left blank. 
HNF-2238

Revision 0

\section{APPENDIX B}

\section{TRANSFER ISSUES}

The following is a compilation of issues that are related to the transfer of waste to the Private Contractors facilities and related staging transfers that support Tank Waste Remediation System (TWRS) operations. Transfers scheduled to occur between 1/1/1998 and 12/31/2011 involving tanks in the 241-AW Tank Farm have been evaluated.

\section{General}

Issue: Current risk and RAM analysis efforts are not yet complete.

Discussion: Risks associated with the transfers are being developed along with a viable Operations and Maintenance (O\&M) concept for TWRS. This new O\&M Concept will include activities related to Waste Feed Delivery (WFD). Until this work is completed, project activities cannot be completely validated.

Issue: Engineering studies which are aimed at optimizing the WFD system are not yet complete.

Discussion: Several studies that have been identified for fiscal year (FY) 1998 are not yet begun. The outcome of these studies could impact the decisions made in identifying scope for completing the design of the WFD system. These studies include an alternative piping route from AN to AP other than the currently scoped W-314 effort. Also included is the control system integration study.

Issue: The transfer schedule identified in the Tank Waste Remediation System Operation and Utilization Plan (TWRSO\&UP) (Kirkbride et al. 1997) reflects staging transfers that are not agreed to by TWRS operations. This means that operations does not feel confident several of the identified transfers can be made or funding priority has not been provided such that planning and preparation for the transfers can be performed.

Discussion: An effort must be made to gain operations agreement to the transfer schedule that will extend through FY 2000. This agreement will help establish priorities for project activities and define a basis for operations sufficient to ensure successful delivery of Envelope A waste. 
HNF-2238

Revision 0

\section{Transfer Pumps}

Transfers from all six tanks in the 241-AW Tank Farm are scheduled to occur before 2011. Transfer pumps are required to accomplish the transfers. Capability and functionality of the existing pumps in all six of the tanks is questionable. The following specific issues have been identified by this evaluation:

Issue: The existing pumps in the AW Farm are either incapable or only marginally capable of delivering feed at the required 1.8 to $2.7 \mathrm{~m} / \mathrm{s}(6$ to $9 \mathrm{ft} / \mathrm{s})$ velocity. This velocity was established as a range for solids transfer in order to maintain a Reynolds number of greater than 20,000 . Supernate transfers could be conducted at a slower rate.

Discussion: A Calculation of the pressure drop for a transfer from 241-AW-101 to 241-AP-102 has been prepared (attached). The calculation shows that even for a relatively short route, the existing pumps are not capable of delivering waste at $0.53 \mathrm{~m} / 3 / \mathrm{min}$ (140 gal $/ \mathrm{min}$ ) (based on a requirement to transfer waste at 1.8 to $2.7 \mathrm{~m} / \mathrm{s}(6 \mathrm{to} 9 \mathrm{ft} / \mathrm{sec}$ through 3-in. pipe). The pump to be installed in the tanks under the W-211 Project will be capable of delivering the waste at $0.53 \mathrm{~m}^{3} / \mathrm{min}(140 \mathrm{gal} / \mathrm{min})$ for even the longest routes in the 200 East Tank Farms. Of the six tanks in the 241-AW Tank Farm, only tank 241-AW-101 is scheduled to receive a new transfer pump under Project W-211.

Issue: A Pump design is needed which allows periodic preventative maintenance (i.e. pump flushing, recirculation, etc.) to improve pump reliability.

Discussion: Waste in some of the tanks consists of saturated salt solution. Crystals grow on the rotating parts of the pump until the shaft cannot be turned. Other tanks contain relatively large volumes of solids. Either crystal growth or depositions of other solids are likely causes of premature pump failure. According to the Pump Cognizant Engineer, after a pump has been idle in a tank for a few years there is about a 50 percent chance the pump will operate successfully.

The current system is not designed to allow flushing or operation of the pumps in a recycle mode. Since the waste in some of the tanks is highly soluble a flush system would likely be capable of dissolving the crystals and allowing the pump to operate. Additionally, a return leg on the piping system from the pump would allow the pump to be operated routinely. Routine operation of the pumps would likely prevent or at least limit crystal growth and ensure operation of the pumps when needed for a transfer.

Issue: The existing transfer pump in tank 241-AW-104 has failed - a replacement has not been identified

Discussion: The transfer pump in 241-AW-104 is broken, and not currently scheduled to be repaired or replaced. Two transfers from tank 241-AW-104 are scheduled in October 1998 and no pump has been identified. This tank will directly support operations surrounding 241-AZ-101. 
HNF-2238

Revision 0

Batch 5 of Envelope A includes 3.83 ML (1,000,000 gal) of waste from 241-AW-104. No transfer pump has been identified in tank 241-AW-104 to make the required transfer.

Issue: The existing pumps in most of the tanks are stick type pumps.

Discussion: Per the Tank Farms Pumping Equipment List (Riesenweber 1992), most of the pumps involved in the transfers listed above are stick type pumps. These pumps are not appropriate because the intake is near the bottom of the tank and the pumps will tend to entrain solids. Nearly all of the transfers are intended to include liquids with only very limited solids content. Transfer using the existing pumps.

Issue: The Design of the Existing Supernate Flex/Float Pumps Causes Pump to Lose Prime if Transfer is Interrupted.

Discussion: The existing flex/float pumps are designed with the impellers above the tank bottom. Once the liquid level in the tank goes below the impellers, pump prime will be lost if the transfer is interrupted. The tank or at least the pump would need to be reflooded at least to the impeller level prior to finishing the transfer.

\section{Mixer Pumps}

Issue: Crust Softening/Removal Techniques are not Tested

Discussion: Crust softening prior to retrieval of waste from Tank 241-AW-101 has been identified as a necessary step in the transfer process. It is not clear that the identified method of adding diluent to top of the tank will effectively soften the crust. Testing of this method with simulated waste should be done to determine if softening is effective. Further, the conceptual crust softening method may be impractical due to tank volume constraints.

\section{Transfer Routings}

Approximately 180 individual transfers involving approximately 30 different transfer routings (see attached route list and sketches of the AW Tank Farm transfer routings) are scheduled over the next 13 years to transfer waste either to or from tanks in the 241-AW Tank Farm. Most of the transfer routes pass through either one or both of the 241-AW-A or 241-AW-B Valve Pits. The routings include at least six configurations involving six of the nozzles in the AW-A Valve Pit and at least six configurations involving at least five of the nozzles in the AW-B Valve Pit. Most of the nozzles used in each pit are involved in two or more of the configurations.

Issue: The current jumper system in valve pits $\mathrm{AW}-\mathrm{A}$ and $\mathrm{AW}-\mathrm{B}$ is inadequate to support transfers 
Discussion: The current jumper configuration in the 241-AW-A and 241-AW-B is inadequate to support the identified transfers. Nozzle to nozzle rigid or flex jumpers without valves would be the least costly in terms of fabrication and materials. The number of configurations needed to support the identified transfers however would require frequent pit entry and jumper modifications. The following nozzles need to be connected in the 241-AW-A Valve Pit:

Nozzle L1 to Nozzles L2, L14, L15, and L16

Nozzle L2 to Nozzles L14, L15, and L19

Nozzle L19 to Nozzle L16

The following nozzles need to be connected in the 241-AW-B Valve Pit:

Nozzle R1 to Nozzles R14 and R15

Nozzle R15 to Nozzle R14, R16, and R19

Nozzle R14 to Nozzle R19

Nozzle R3 to Nozzle R9.

A suggested solution would be to design a manifold jumper system with the transfer requirements specifically in mind. A manifold system would require only valving changes to reconfigure the routing through the valve pit. The manifold system might be combined with a flush, caustic injection, and/or recycle system which would allow routine operation of the pumps (Conceptually a manifold system is not significantly different than the Project W-454 design.)

Introduction of valves in a.manifold system may result in more point failures than the current system; however, more pit entries that would be required for configuration changes which support a system of flex jumpers.

Another option may be to design a better method of making jumper changes such as a vehicle with necessary confinement structures built in and a light duty (10 ton) crane. The concept would be to move the vehicle into place, open the pit, make the necessary jumper change, and close the pit all remotely. A similar design was developed for the hot conditioning annex of the canister storage building (Hot Conditioning System Equipment Conceptual Design Report [Merrick and Associates and W. L. Willis 1996], Drawing SK2-2-300420).

The nozzle connections listed were developed using the most likely (shortest available 3 -in. routing) routings from the source tank to the destination tank. Additionally, for the return of slurry from the evaporator, the following connection in the 2-in. slurry lines needs to be made:

Nozzle R3 needs to be connected to Nozzle R9. There is currently a jumper that connects Nozzle R3 to Nozzle R9.

Issue: Current Jumper System in Pump Pit 241-AW-02A is inadequate to support transfers. 
HNF-2238

Revision 0

Discussion: The current Jumper configuration in Central Pump Pit 241-AW-02A does not allow a transfer from Tank 241-AW-102 to any other tank in the system without passing through the 242-A Evaporator. A jumper modification is needed to allow connection of Nozzle A to Nozzle $\mathrm{K}$ in the 241-AW-02A. This modification will allow transfer of waste from tank 241-AW-102 to other tanks without passing the waste through the evaporator.

Issue: Line 3-in. $\mathrm{SN}-219-\mathrm{M} 25$ is not operable.

Discussion: Line 3-in. SN-219-M25 has clean-out boxes. The clean-out boxes have leaked in the past and no permanent fix has been identified. The 3 -in. SN-219 connects the 241-A-B valve pit with the 241-AW-B valve pit. There is an available transfer route between the 241-A-B and 241-AW-B valve pits as follows:

Through line 3-in. SN-204 from valve pit 241-A-B to valve pit 241-A-A

Through line 3-in. SN-220 from Valve Pit 241-A-A to Valve Pit 241-AW-A

Through line 3-in. SN-271 from Valve Pit 241-AW-A to. Valve Pit 241-AW-B.

Issue: Line 2-in. SL-161-M25 is not operable.

Discussion: Acid was transferred from the PUREX Facility to tank 241-AW-101 in 1984 or 1985. The transfer route used Line 2-in. SL-161-M25, a carbon steel pipe. The line failed a pressure test on 04/16/1985 and was taken out of service at that time. The transfer path may also have gone through Line 3-in. SN-271-M25, the supernate transfer line between the AW-A and the AW-B Valve Pits. If the acid transfer routing did include the supernate cross tie line, the 3-in. SN-271-M25 line may be damaged as well.

Issue: Abandonment of the 241-A-A Valve Pit requires that line LIQW-702 be connected directly to line SN-220.

Discussion: The proposed new W-314 line does not require the use of Valve Pit 241-A-A excepting waste transferred from 204-AR. A route from 204-AR to 241-AW that bypasses Valve Pit 241-A-A would allow this valve pit to be abandoned. Connect line LIQW-702 directly to line SN-220, bypassing the 241-A-A Valve Pit.

\section{Regulatory Compliance}

Issue: Compliance of the Transfer Lines with WAC 173-303 Dangerous Waste Regulations.

Discussion: The configurations of some existing piping - pit wall penetrations do not meet secondary containment requirements for tank systems because they do not ensure drainage of pipe leaks to a leak collection and removal system. Current agency agreements on other facilities (e.g., 242-A Evaporator) call for repairing/upgrading lines if unit is being modified for any other reason. Agreements with agencies may be needed if waste transfers will occur through lines with non-compliant wall penetrations. 
HNF-2238

Revision 0

This compliance issue includes most of the lines in the AW Tank Farm.

All non-compliant lines need to be identified. Once the complete list of non-compliant lines has been generated an alternative generation and analysis process should be used to develop a strategy for dealing with each non-compliant line.

Issue: Concrete line Encasements

Discussion: Concrete encasements have not been demonstrated to meet secondary containment requirements. Pipelines without compliant secondary containment must be leak tested on an annual basis. All non-compliant lines need to be identified. Once the complete list of noncompliant lines has been generated an alternative generation and analysis process should be used to develop a strategy for dealing with each non-compliant line.

\section{Issue: Ancillary Equipment Secondary Containment}

Discussion: Secondary containment for ancillary equipment (pits, diversion boxes, pipe trenches) must prevent migration of waste to the soil. This can be accomplished by leak testing the secondary containment. The Washington State Department of Ecology also believes these units should have coatings, but a regulatory basis for this requirement has not been found. Alternatively, an inspection schedule might be negotiated to demonstrate the condition of these units.

Issue: Written Integrity Assessment

Discussion: A written integrity assessment is required for operation of a dangerous waste tank system. A limited amount of integrity assessment work has been performed for DSTs and transfer systems. Negotiation of integrity assessment scope for TWRS facilities is ongoing.

\section{Safety}

Issue: System upgrades may be required to support Authorization Basis/BIO

Discussion: The document Authorization Basis Assessment of Waste Feed Delivery (Grams 1997) provides an evaluation of the Nuclear Safety Licensing needs for waste feed delivery. The evaluation was performed using the USQ process as a guide. Thus, the evaluation identifies activities that either require revisions to be included within the Authorization Basis or an Authorization Basis amendment to include the activities as authorized activities.

The first issue is the identification of activities or topics that could not be adequately evaluated at this time due to insufficient information. These activities or topics include the following: 
HNF-2238

Revision 0

- $\quad$ Returned waste eluent from waste vitrification facilities

- Return and interim storage of ion exchange media

- Waste transfer to contractor's tanks

- Maintenance of equipment critical to waste feed delivery.

The second issue is the identification of activities which have the potential for significantly changing source terms and resulting accident consequences, both radiological and toxicological. These activities include the following:

- Waste mobilization with mixer pumps

- Waste conditioning for transfer

- Transfers of slurries

- $\quad$ Sludge washing (caustic and water based)

- Waste feed adjustment (shimming).

Follow-on tasks to complete the evaluation of waste feed delivery activities have been identified. These tasks include a HAZOP, evaluation of source terms, evaluation of aerosol generation, criticality analysis, analysis of impact on the potential for tank bump, and evaluation of transfers from tank 241-SY-101. The results of these evaluations could either require revising the activities such that they are covered by the current Authorization Basis or an Authorization Basis amendment to include the activities as authorized activities. These issues need to be fully developed, and may lead to the need for additional engineered mitigation systems.

Issue: It will be dificult to isolate malfunctioning systems from systems that are functioning because the BIO does not accept valves as an appropriate means of isolating active transfer equipment from inactive systems.

Discussion: In transfers made in the course of Tank Farms operations prior to the implementation of the Basis for Interim Operation, using closed valves (i.e., double valve isolation) was an acceptable means of isolating active transfer equipment. In the analysis performed for the BIO, double valve isolation was not considered an effective method of preventing or mitigating leaks induced by active transfer equipment.

The main reason for not allowing double valve isolation was the fact that misroutings can occur and indeed have occurred in the past. To understand which controls, and safety systems, structures, and components (SSCs) need to be operable and in place prior to waste transfers it is necessary to understand the control philosophy that has been constructed related to waste transfers. The following control strategy for potential misrouting of waste transfers is described in the BIO:

The selected safety SSC and TSR controls for waste transfer-related accidents provide three levels of defense to prevent or mitigate the risk of these accidents caused by waste transfer misrouting. 
The first level of defense requires that the controls selected to prevent or mitigate leaks from waste transfer systems (e.g., transfer system covers, transfer leak detection systems, periodic ground-level radiation surveys of single-walled, direct-buried/bermed lines) are operable or implemented for the "physically connected" topography of the waste transfer. The physically connected topography refers to piping, structures, and tanks and their associated instrumentation as follows.

1. Physically connected piping is any piping which is part of or connected to the transfer route. Piping need not be considered connected to the transfer route if it is physically disconnected by a removal of piping (i.e., air gap) or isolated with a blind flange/process blank. (Note: Closed valves do not physically disconnect piping.)

The East/West cross-site transfer line is considered physically connected piping only when cross-site waste transfers are in progress. The East/West cross-site transfer line is the piping between 241-UX-154 diversion box and 241-ER-151 diversion box.

An operable service water pressure detection system is considered to physically disconnect piping on either side of the detection system.

2. Physically connected structures are those structures through which physically connected piping runs, or structures that could be subject to leakage from physically connected piping.

3. Physically connected tanks are those tanks connected to the transfer route, those tanks connected to the physically connected piping, and those tanks designed to receive leakage from physically connected piping through a drain path.

This first level of defense ensures that, should a mistransfer occur because of a leaking or misaligned isolation valve, controls are operable and implemented where the waste could be misrouted to detect and/or mitigate the consequences of a potential waste leak (i.e., spray, pool, underground plume). For example, the transfer leak detection systems must be operable in all physically connected structures (i.e., process pits, diversion boxes, valve pits, and clean out boxes [COBs] ) during the waste transfer.

The second level of defense is the selection of additional controls for identifying waste transfer leaks or misroutings. These controls are the TSRs for periodic material balances and periodic monitoring of interconnected tanks during waste transfers. Upon indication of a significant material balance discrepancy or unexpected tank level rise, the waste transfer would be shutdown to the limit the potential quantity of waste leaked or misrouted.

The third level of defense is controls that prevent waste transfer misrouting. These TSR controls include the following. 
1. Wherever practical, isolation of piping connected to the planned waste transfer path with two closed valves in series.

2. Independent verification of the planned waste transfer route.

3. Waste transfer system and jumper configuration control.

4. Waste transfer system operations by approved procedures.

These controls reduce the likelihood of misrouting during planned waste transfers.

In summary, the selected safety SSCs and TSR controls and their required application during waste transfers provide diversity and multiple levels of defense. The controls minimize the likelihood of a waste transfer misrouting and ensure that, should a waste leak occur as a result of a misrouting, mitigative safety SSCs or TSRs are in place to reduce the accident consequences.

Because transfers involving virtually all of the DSTs and many of the SSTs are scheduled to occur, the "physically connected" system is likely to become very large and quite complex. The size and complexity of the system will likely reduce system availability.

\section{Instrumentation/Ancillary Equipment}

Issue: Automatic Liquid Level Detection Availability

Discussion: Automatic liquid level detection is not available in tank 241-AW-101 and some other tanks. Part of the system that detects misroutings is the liquid level detection. Since some of the tanks liquid level detection is manual, the ability to properly monitor and control transfers is jeopardized.

\section{Issue: Equipment/Instrument Control}

Discussion: Equipment and instrumentation control in the 241-AW Tank Farm is via local control. System upgrades are needed to ensure that the control system for the AW Tank Farm is compatible with systems used in other farms.

\section{Issue: Current System Complexity}

Discussion: The number of valves involved in the transfers (MOVs Three way, and Isolation) may increase the risk of misroutings and valve failures. Additionally, centralized control and the master pump shutdown system may result in low system availability. The current operations/maintenance philosophy may need to be revisited in light of the large number of 
transfers required and the impacts associated with the failure of the system to perform as required. Modifications such as new simplified jumpers and methods of isolating parts of the system entirely may be beneficial and should be evaluated.

Issue: Integration requirements for new and existing instrumentation and controls have not been established.

Discussion: Existing instrumentation and control systems need to interface with new systems. The systems need to work in a coordinated way per the operations concept. Existing and new monitoring and control systems will be operated by TWRS personnel for efficiency, all of the farms should be similar to the extent practicable (best engineering practice). Specific I\&C integration requirements will be developed under an existing task by Vista Research.

\section{Ventilation System}

Issue: Electrical supply and control circuits to the primary fans are in poor condition and jeopardize the reliability of the system.

Discussion: Specific components are identified in the Condition Assessment Survey (Golberg 1996). The primary issues were that wire terminations are not properly supported and control circuits are not fully functional. LCO 3.2.1 Technical Safety Requirements (BIO) requires that the active primary ventilation system shall be operable. The basis for this requirement is the need to prevent flammable gases from accumulating in the tank headspace. Suggested scope is to write a work plan to confirm condition of electrical supply and control circuits and document specific components requiring repair or replacement.

Issue: The 241-AW primary ventilation stack will likely require upgrades to provide continuous air emissions monitoring in compliance with NESHAP.

Discussion: It is likely that the 241-AW primary ventilation stack will be redesignated from a minor to a major stack. Permitting, monitoring, and potentially treatment requirements will change. It will be necessary to upgrade the 241-AW ventilation system to be compliant with NESHAP. This is likely to require changes to the effluent monitoring system. A fully NESHAP - compliant ventilation stack system has been developed under Project W-420. The estimated project cost for a single stack is approximately $\$ 200,000$. A criterion for designation of an effluent discharge stack as minor or major is whether the potential for unabated discharges results in an off-site exposure of $0.1 \mathrm{mrem} / \mathrm{yr}$. Air modeling requirements have recently changed by a factor of 1.5 . This increased factor causes the current 241-AW primary stack to exceed $0.06 \mathrm{mrem} / \mathrm{yr}$ unabated releases under current conditions. Increased releases are likely due to mixer pump operation. The design for changes to the 241-AW primary ventilation stack will be similar to the design done for the W-420 Project. 
Issue: The current HVAC system does not have the capability of removing toxic pollutants from off-gas.

Discussion: This issue is particularly important during a GRE. The concentrations of volatile toxic pollutants in the tank waste are not well characterized. Release limits are not currently defined. Install or provide expansion capability for an activated carbon filter and dry scrubber system in the HVAC system to reduce or eliminate toxic pollutants from the off-gas stream. Toxic constituents are present in off-gas from the tanks potentially at concentrations which will require abatement. This follows the recommendation made in the Initial Assessment Report HVAC Systems (Kriskovich 1996). Adding the capability of toxic constituent removal to the HVAC systems is currently planned for W-314. Development of a basis for this requirement will require vapor space sampling of target DSTs with analysis for TAPs.

Issue: There currently is no direct method to measure the primary ventilation airflow from tank 241-AW-101.

Discussion: The filtered inlet air flowrate is monitored but this doesn't account for inleakage through pump pits, etc. It is necessary to install flow meters in tank 241-AW-101 primary ventilation exhaust ducting. Flow monitoring in conjunction with hydrogen monitoring equipment already installed, will allow for determination of the overall hydrogen generation and release rates. This information will be necessary for controlling the waste degassing operation. See Technical Basis For Installation of the Double-Shell Tank Exhaust Flow Monitoring Systems (Willingham 1997a) for a detailed basis and Double-Shell Tank Primary Ventilation Exhaust Flow Monitor System Design Description (Willingham 1997b) for conceptual design information.

\section{Electrical}

Issue: A 1995 assessment found many deficiencies with the motor control center MCC-241-AW.

Discussion: This MCC will be operational through the end of the Phase 1 Privatization so the electrical system must be repaired. A work package will be prepared to confirm the condition of MCC-241-AW and document the specific components requiring repair or replacement.

Issue: The addition of waste retrieval systems to tank 241-AW-101 will add a significant load to the 241-AW farm electrical system.

Discussion: It is not clear that the new loads that will be installed under project W-211 have been fully evaluated and compared with excess capacity in the system. The electrical transmission systems may need to be upgraded to account for the new loads. A work package will be developed to review the electrical system capability and availability versus use and document. 
HNF-2238

Revision 0

\section{Utilities}

Issue: Transfer line flush requirements have not be established.

Discussion: Water is needed for transfer line flushing and dilution/dissolution of waste. The current flush system at $241-\mathrm{AW}$ is capable of approximately $0.23 \mathrm{~m} / \mathrm{min}(60 \mathrm{gal} / \mathrm{min})$. It is expected that the new system to be installed by project W-211 will provide up to $0.57 \mathrm{~m} 3 / \mathrm{min}$ (150 gal $/ \mathrm{min})$. Establishing a defensible basis for the flowrate will require a review of solids resuspension models and potentially pipe loop studies. A work plan and cost estimate for these activities are currently being developed by PNNL.

Issue: The 241-AW air compressors cycle frequently resulting in early equipment failures.

Discussion: The air compressors cycle every 30 to 50 seconds and experience early failures. Reliability of some of the tank farm instrumentation is based on the reliability of the compressed air system and are needed to support existing and future operations. A work package will be developed to assess the compressed air system and determine the cause of the frequent cycling. After the cause of the cycling has been determined, a scoping study of alternatives is needed to develop a project requirement. Alternatives might include repair or replacement of buried air lines if leaking or larger compressed air receiver tanks.

\section{Waste Retrieval}

Issue: Process requirements have not been completed for retrieval of waste from tank 241-AW-101.

Discussion: The process requirements for retrieval of waste from tank 241-AW-101 as feed to the privatization vendors under Phase I of the contract have not be finalized. These requirements primarily establish the design criteria and bases for the criteria for project W-211. A number of process requirement issues are provided in Table E-7 of Appendix E.

\section{OPPORTUNITIES}

The most likely transfer route to tanks 241-AP-102 and 104 flows from the north tank farms in the 200 East Area south through new lines to be provided by Project W-314. The likely route passes through several valve and pump pits and numerous valves. A failure of a component in the likely transfer route may lead to a delay in delivery of feed to the private contractor with the associated "failure to deliver" penalties. 
HNF-2238

Revision 0

The likely transfer route from tank 241-AW-101 to tanks 241-AP-102 and 104 uses a different set of lines than the likely route from 241 AN Farm. Tank 241-AW-101 is currently scheduled to be delivered as the third of five tanks in Envelope A. By preparing necessary transfer systems for tank 241-AW-101 earlier than the current W-211 schedule, and then rearranging the schedule such that tank 241-AW-101 is delivered at the end of envelope A, the DOE and TWRS contractor gain a higher assurance that feed delivery schedules can be met; and failure to deliver penalties avoided. 
HNF-2238

Revision 0

This page intentionally left blank. 
HNF-2238

Revision 0

\section{APPENDIX C}

\section{TRANSFER REQUIREMENTS AND EXPECTATIONS}


HNF-2238

Revision 0

This page intentionally left blank.

C-2 
HNF-2238

Revision 0

\title{
APPENDIX C \\ TRANSFER REQUIREMENTS AND EXPECTATIONS
}

\begin{abstract}
Appendix $\mathrm{C}$ contains a list of requirements and expectations for the transfers. The transfers were divided into three groups. The first group of transfers is the waste feed delivery transfers. The second group is one slurry transfer to clean the solids from tank 241-SY-102. The third group is all remaining transfers. The following assumptions were made
\end{abstract}

- $\quad$ Slurry transfers have typically been made through 2 -in. lines, whereas supernatant liquids have typically transferred through 3 -in. lines. The transfer velocity through a 2 -in. Line is over twice as high as through 3 -in. line for the same volumetric flow rate. The higher velocity helped to ensure that the solids in the slurry would not settle during the transfer and cause line pluggage. A guidance has been established that if a transfer could be done such that a Reynolds Number $\left(\mathrm{Re}_{\mathrm{d}}\right)$ greater than 20,000 was achieved, no solids settling or line pluggage would occur. In 3-in. line, slurry with a density of $1.41 \mathrm{~g} / \mathrm{ml}$, and a viscosity of $10 \mathrm{cP}$ would need to be transferred with a velocity of at least $1.83 \mathrm{M} / \mathrm{sec}$. In order to achieve a $\mathrm{Re}_{\mathrm{d}}$ greater than 20,000 . It is assumed that this guidance only applies to slurry transfers. For supernatant transfers (which contain only liquids) there is no minimum $\mathrm{Re}_{\mathrm{d}}$ guidance. It will be important to demonstrate that no solids will form during supernatant transfers due to temperature or concentration changes which will occur during waste dilution or transfer.

- Of the approximately 180 total transfers to or from the AW Tank Farm Tanks, only transfers from tank 241-AW-101 to tanks 241-AP-102 and 104 (four transfers), and transfers from tank 241-SY-102 to tank 241-AW-105 (one transfer) are slurry transfers. All of the other approximately 175 transfers involve only the transfer of liquids.

- Evaporator campaigns will not concentrate the waste to the point that the waste is considered a slurry. All waste transfers from the evaporator to tank 241-AW-106, and from tank 241-AW-106 to other tanks will therefore consist only of liquids.

- The W-211 Project will provide mixer pumps or an equivalent and new transfer pumps capable of meeting the pump-ability rule $\left(\mathrm{Re}_{\mathrm{D}}=20,000\right)$ in tanks 241-AW-101. This installation is scheduled to occur prior to the transfers.

- Tanks will be sampled prior to transfers and tank contents shown to be compatible. 
HNF-2238

\section{Revision 0}

- The Tank Farms has the capability and will maintain the capability to obtain tank samples from different levels in all tanks. 
Table C-1. Transfer Process Needs.

\begin{tabular}{|c|c|c|c|c|}
\hline Waste transfer set & Process needs & Basis & $\begin{array}{l}\text { Equipment } \\
\text { requirements }\end{array}$ & Comments/issues \\
\hline \multirow[t]{3}{*}{$\begin{array}{l}\text { Envelope A } \\
\text { (Slurries/ } \mathrm{H}_{2} \text { Watch List) }\end{array}$} & 1. Degas the tank contents. & $\begin{array}{l}\text { 1. Envelope A tanks are all } \\
\text { flammable gas watch list } \\
\text { tanks which require } \\
\text { degassing prior to transfer } \\
\text { per the HNF-1985 (Draft). }\end{array}$ & $\begin{array}{l}\text { 1. Equipment is needed to } \\
\text { provide mixing of tank } \\
\text { contents such that degassing } \\
\text { can be achieved. }\end{array}$ & $\begin{array}{l}\text { 1. The extent of mixing } \\
\text { required must be determined } \\
\text { (i.e., Solids must be stirred } \\
\text { adequately to release gas } \\
\text { bubbles). The tank } 101-\mathrm{SY} \\
\text { degassing results may be } \\
\text { used as a basis. }\end{array}$ \\
\hline & $\begin{array}{l}\text { 2. Dissolve } 100 \% \text { of soluble } \\
\text { solids. }\end{array}$ & $\begin{array}{l}\text { 2. } 100 \% \text { of the soluble } \\
\text { solids must be dissolved to } \\
\text { assist in achieving the } \\
\text { maximum order quantity } \\
\text { (HNF-2168). }\end{array}$ & $\begin{array}{l}\text { 2. Equipment is needed } \\
\text { which provides for the } \\
\text { addition of solvent and } \\
\text { mixing/dissolution of residual } \\
\text { soluble salts. Equipment is } \\
\text { also required to inspect the } \\
\text { tank for residual solids. }\end{array}$ & $\begin{array}{l}\text { 2. An effective means to } \\
\text { remove large deposits of } \\
\text { solids on tank walls and } \\
\text { protruding equipment (i.e., } \\
\text { Air lift circulators, } \\
\text { thermocouple trees) has not } \\
\text { been demonstrated. }\end{array}$ \\
\hline & 3. Monitor tank contents & $\begin{array}{l}\text { 3. The tank level and density } \\
\text { must be monitored to ensure } \\
\text { process control and } \\
\text { compliance with safety based } \\
\text { maximum and minimum tank } \\
\text { liquid level is per HNF-1985 } \\
\text { and TWRSO\&UP. In } \\
\text { addition, tank AW-104 } \\
\text { (which contains insoluble } \\
\text { solids) contents must be } \\
\text { monitored so that the } \\
\text { insoluble solids fraction in } \\
\text { the retrieved supernate does } \\
\text { not exceed } 5 \text { vol \% } \\
\text { (TWRSO\&UP). }\end{array}$ & $\begin{array}{l}\text { 3. Automatic tank level and } \\
\text { SpG instrumentation must be } \\
\text { provided for a range of } 0- \\
100 \% \text { of tank volume and } 1 \text { - } \\
1.5 \mathrm{SpG} \text {. Instrumentation } \\
\text { must be provided in tank } \\
104-\mathrm{AW} \text { to measure the } \\
\text { solids content of the } \\
\text { supernatant. }\end{array}$ & $\begin{array}{l}\text { 3. Instrumentation must be } \\
\text { resistant to solid deposition } \\
\text { or a means of cleaning, } \\
\text { purging or flushing provided. } \\
\text { An interlock is needed to } \\
\text { activate the master pump } \\
\text { shutdown prior to reaching } \\
\text { the tank level (See Table A-2 } \\
\text { in TWRSO\&UP). An } \\
\text { interlock is needed to } \\
\text { shutdown the decant pump } \\
\text { when the liquid level reaches } \\
10 \text { inches (TWRSO\&UP), } \\
\text { and/or if the solids content } \\
\text { exceeds } 5 \text { vol \%. }\end{array}$ \\
\hline
\end{tabular}




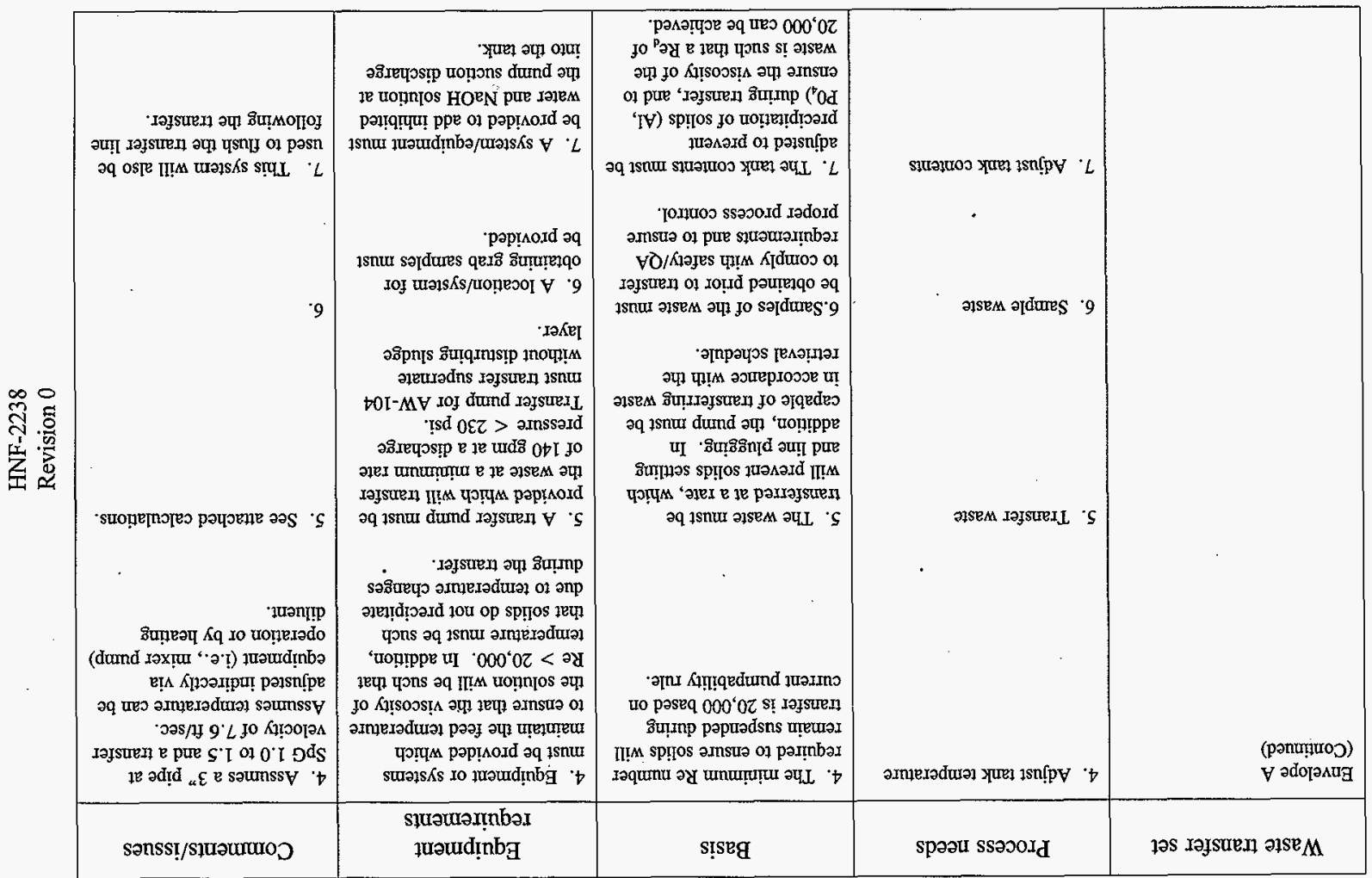

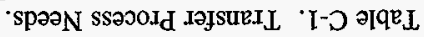


Table C-1. Transfer Process Needs.

\begin{tabular}{|c|c|c|c|c|}
\hline Waste transfer set & Process needs & Basis & $\begin{array}{l}\text { Equipment } \\
\text { requirements }\end{array}$ & Comments/issues \\
\hline $\begin{array}{l}\text { Envelope A } \\
\text { (Continued) }\end{array}$ & 8. Flush Transfer Lines. & $\begin{array}{l}\text { 8. Lines must be flushed } \\
\text { (raw water) with an } \\
\text { equivalent volume } 1.5 \text { times } \\
\text { the transfer length to remove } \\
\text { any static waste. }\end{array}$ & $\begin{array}{l}\text { 8. The equipment is } \\
\text { assumed to be incorporated } \\
\text { with the transfer system. }\end{array}$ & $\begin{array}{l}\text { 8. The flushing rate must be } \\
\text { the same as the transfer rate } \\
\text { to remove any solids that } \\
\text { may precipitate or settle. It } \\
\text { is assumed that the impact of } \\
\text { precipitation due to flushing } \\
\text { is negligible. }\end{array}$ \\
\hline $\begin{array}{l}102 \mathrm{SY} \\
\text { (Non Soluble Solids) }\end{array}$ & $\begin{array}{l}\text { 1. Monitor the tank } \\
\text { contents. } \\
\text { 2. Adjust the tank } \\
\text { temperature. } \\
\text { 3. Transfer the waste. }\end{array}$ & $\begin{array}{l}\text { 1. The tank level and density } \\
\text { must be monitored to ensure } \\
\text { process control and } \\
\text { compliance with safety based } \\
\text { on maximum and minimum } \\
\text { levels per HNF-1985 and } \\
\text { TWRSO\&UP. } \\
\text { 2. The minimum Re number } \\
\text { required to ensure solids will } \\
\text { remain suspended during } \\
\text { transfer is } 20,000 \text { per } \\
\text { pumpability rule. } \\
\text { 3. The waste must be } \\
\text { transferred at a rate, which } \\
\text { will prevent solids settling } \\
\text { and line plugging. }\end{array}$ & $\begin{array}{l}\text { 1. Automatic tank level and } \\
\text { SpG instrumentation must be } \\
\text { provided for a range of } 0- \\
100 \% \text { of tank volume and } 1 \text { - } \\
1.5 \text { SpG. } \\
\text { 2. Equipment or systems } \\
\text { must be provided which } \\
\text { maintain the feed temperature } \\
\text { to ensure that the viscosity of } \\
\text { the solution will be such that } \\
\text { Re }>20,000 \text {. In addition, } \\
\text { temperature must be such } \\
\text { that solids do not precipitate } \\
\text { due to temperature changes } \\
\text { during the transfer. } \\
\text { 3. A transfer pump will be } \\
\text { provided which will transfer } \\
\text { the waste at a route of } 140 \\
\text { gpm at a discharge pressure } \\
\text { within the limits of the cross- } \\
\text { site transfer line. }\end{array}$ & $\begin{array}{l}\text { 1. A means of cleaning or } \\
\text { purging the instrumentation } \\
\text { needs to be provided. } \\
\text { 2. Assuming a } 3 \text { " pipe at a } \\
\text { SpG of } 1.0 \text { to } 1.5 \text { and a } \\
\text { transfer velocity of } 7.6 \\
\text { ft/sec. Assumes temperature } \\
\text { can be adjusted indirectly via } \\
\text { equipment (mixer pump) } \\
\text { operation or by heating } \\
\text { diluent. }\end{array}$ \\
\hline
\end{tabular}


Table C-1. Transfer Process Needs.

\begin{tabular}{|c|c|c|c|c|}
\hline Waste transfer set & Process needs & Basis & $\begin{array}{l}\text { Equipment } \\
\text { requirements }\end{array}$ & Comments/issues \\
\hline \multirow[t]{4}{*}{$\begin{array}{l}102 \mathrm{SY} \\
\text { (Continued) }\end{array}$} & 4. Adjust the tank contents & $\begin{array}{l}\text { 4. The tank contents must be } \\
\text { adjusted to prevent } \\
\text { precipitation of solids (Al, } \\
\mathrm{PO}_{4} \text { ) during transfer. }\end{array}$ & $\begin{array}{l}\text { 4. A system/equipment must } \\
\text { be provided to add inhibited } \\
\text { water and } \mathrm{NaOH} \text { solution at } \\
\text { the pump suction and also } \\
\text { discharge into the tank. }\end{array}$ & $\begin{array}{l}\text { 4. This system will also be } \\
\text { used to flush the transfer line } \\
\text { following the transfer. }\end{array}$ \\
\hline & 5. Sample the waste. & $\begin{array}{l}\text { 5. Samples of the waste } \\
\text { must be obtained prior to } \\
\text { transfer to comply with } \\
\text { safety/QA requirements and } \\
\text { to ensure proper process } \\
\text { control. }\end{array}$ & $\begin{array}{l}\text { 5. A location/system for } \\
\text { obtaining grab samples must } \\
\text { be provided. }\end{array}$ & $\begin{array}{l}\text { 5. Assumes a mixer pump } \\
\text { can meet the requirements. }\end{array}$ \\
\hline & 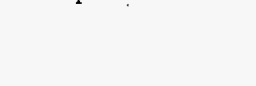 & $\begin{array}{l}\text { 6. Equipment must be able } \\
\text { to mobilize the solids. }\end{array}$ & $\begin{array}{l}\text { provided which will ensure } \\
\text { that the solids can be } \\
\text { mobilized. }\end{array}$ & . \\
\hline & 7. Flush Transfer Lines. & $\begin{array}{l}\text { 7. Lines must be flushed } \\
\text { (raw water) with an } \\
\text { equivalent volume } 1.5 \text { times } \\
\text { the transfer length to remove } \\
\text { any static waste. }\end{array}$ & $\begin{array}{l}\text { 7. The equipment is } \\
\text { assumed to be incorporated } \\
\text { with the transfer system. }\end{array}$ & $\begin{array}{l}\text { 7. The flushing rate must be } \\
\text { the same as the transfer rate } \\
\text { to remove any solids that } \\
\text { may precipitate or settle. It } \\
\text { is assumed that the impact of } \\
\text { precipitation due to flushing } \\
\text { is negligible. }\end{array}$ \\
\hline
\end{tabular}


Table C-1. Transfer Process Needs.

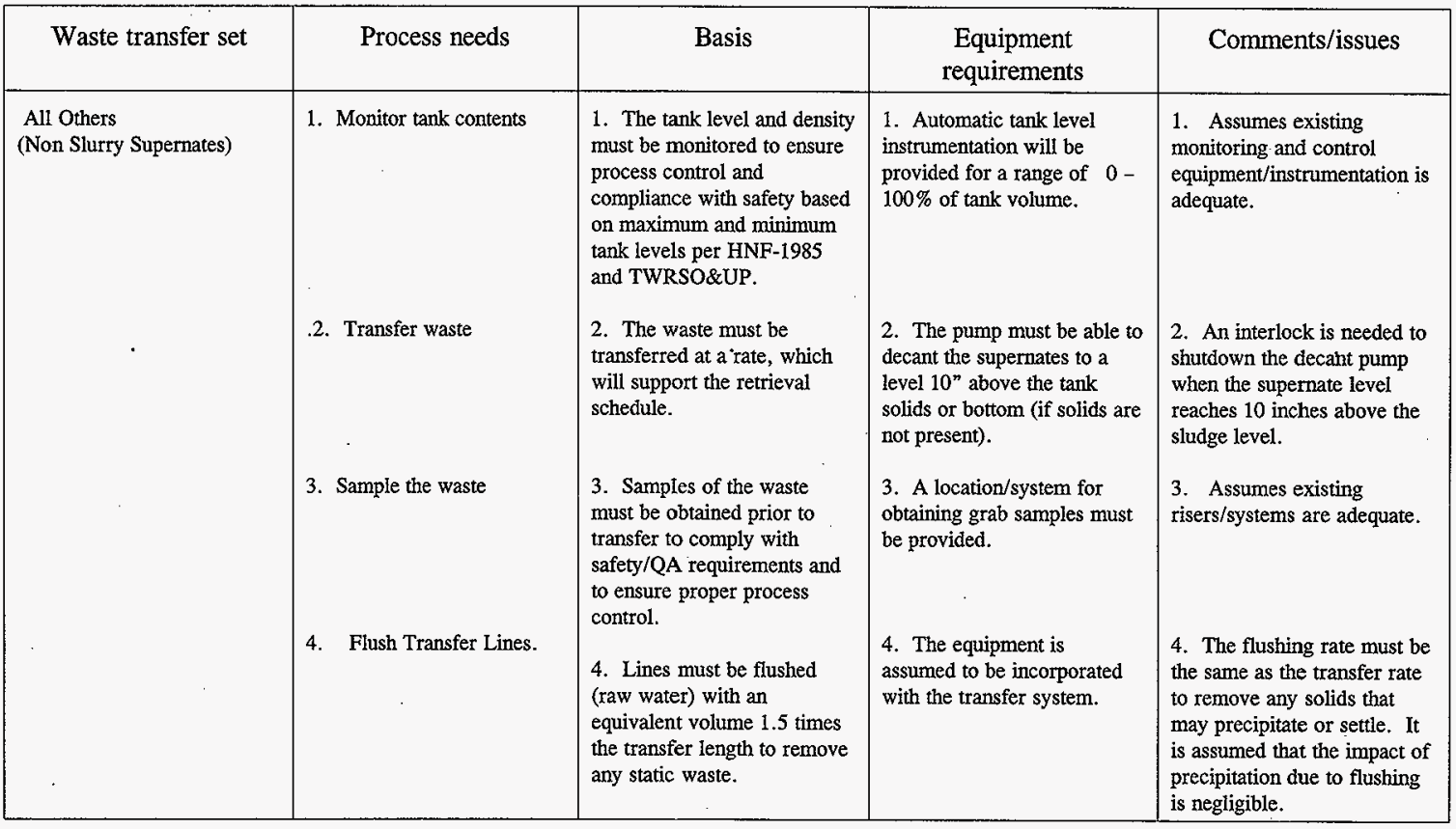


HNF-2238

Revision 0

This page intentionally left blank. 
HNF-2238

Revision 0

\section{APPENDIX D}

\section{CALCULATIONS}


HNF-2238

Revision 0

This page intentionally left blank.

D-2 
HNF-2238

Revision 0

\section{APPENDIX D}

\section{CALCULATIONS}

Appendix D contains calculations done to determine transferability of the waste along the 32 transfer paths identified in Appendix A. Transfers that deliver waste to the private contractor(s) were considered to be of utmost importance. Four of the transfers in the list deliver the diluted contents of tank 241-AW-101 to the private contractor(s) as the third feed tank in Phase 1B Envelope A.

Viscosity, density, and flow rate information is necessary to estimate the pressure drop which occurs as fluids are transferred through pipes. Viscosity and density information on the diluted waste in tank 241-AW-101 is not available, but viscosity and density information for diluted tank 241-AN-105 waste is available. The waste in tanks 241-AW-101 and 241-AN-105 is assumed for the purposes of this document to have similar density and viscosity characteristics. Given the nature of the waste in tanks 241-AW-101 and 241-AN-105, this assumption is conservative because tank $241-\mathrm{AN}-105$ has a higher solids content. The measured viscosities and densities from the tank 241-AN-105 dilution study were used in the pressure drop calculation for the transfer from tank 241-AW-101 to tanks 241-AP-102/104. A requirement has been made (Claghorn 1998) for feed delivery transfers to have linear velocities between six and nine feet per second. A velocity of $1.8 \mathrm{~m} / \mathrm{sec}(6 \mathrm{ft} / \mathrm{sec})$ equates to a flow of approximately $0.53 \mathrm{~m} / 3 / \mathrm{min}(140 \mathrm{gal} / \mathrm{min})$ through 3-in. pipe. A flow rate of $0.53 \mathrm{~m}^{3} / \mathrm{min}$ (140 gal $\left./ \mathrm{min}\right)$ is equivalent to approximately $3800 \mathrm{~m}^{3}(1,000,000 \mathrm{gal})$ over five days. With the viscosity, density and velocity assumed, Reynolds numbers were calculated for three temperatures. The Reynolds numbers were used to determine friction factors and the friction factors were in turn used to estimate pressure drops per unit length of pipe. The equivalent length of pipe of the transfer route from tank 241-AW-101 to tank 241-AW-102 was calculated by summing the equivalent lengths of all of the components in the route. The pressure drop per unit length of pipe was then multiplied by the equivalent length of the transfer route to estimate the total pressure drop in the line. This calculation is included in this appendix.

It is important to note that actual pressure drop in the line is dependant on the actual flow rate, which flow rate is in turn dependant on the pump. Two pump curves were available, one for some existing pumps in the 241-AW Tank Farm, and one for a nextgeneration pump scheduled to be installed as part of Project W-211. It is important to note that the two pump types are likely to represent boundary conditions regarding which pumps will actually be in service when the transfers are made. The pressure drops were calculated for a fluid with a specific gravity of 1.5 and a viscosity of 5 centipoise at flow rates from 0 to $0.85 \mathrm{~m}^{3}$ ( 0 to $225 \mathrm{gal} / \mathrm{min}$ ). These pressure drops were used to calculate total pressure drops in lines with equivalent lengths of from $305 \mathrm{~m}$ to $3050 \mathrm{~m}$ (1,000 to $10,000 \mathrm{ft})$. With the exception of the transfers from tank 241-SY-102, all other transfers evaluated here have equivalent lengths of from approximately $305 \mathrm{~m}$ to $3050 \mathrm{~m}(1,000 \mathrm{ft}$ to $5,000 \mathrm{ft})$. Both 2-in. and 3-in. pipe was evaluated. Curves of flow rates versus pressure drops for the given 
equivalent length were plotted on the pump curves. The pump should operate at the point where the curves intersect, and therefore the expected actual flow rate for the given pump can be determined. For each of the equivalent lengths expected flow rates were determined for two and three inch lines for both pump types. A curve was plotted which gives the expected flow rates for each pump type in 2-in. and 3-in. lines over the various equivalent lengths. A plot of viscosity versus density versus flow rate through three inch pipe for a Reynolds number of 20,000 is also included.

Another important issue evaluated is the total solids to be transferred. It is believed that the PHMC will be limited to $5 \mathrm{vol} \%$ solids in the material delivered to the private contractors. Tank 241-AW-101 currently contains approximately 27 vol\% solids. Most of this material is considered to be soluble. It is important to evaluate what fraction of the material will remain a solid following dilution and dissolution prior to delivery to the private contractors. An Environmental Simulation Program (ESP) ${ }^{1}$ water survey was performed in an attempt to determine solids content with dilution volumes of 25,50 , and $75 \mathrm{vol} \%$. The results indicate that the solids content will be much less than the allowable 5 vol\%.

Another important variable to evaluate is temperature. Some of the tanks contain saturated solutions. As the temperature of the saturated solutions drops, solids form. Tank temperatures were compared to tank contents. As a general rule, tanks that contain dilute solutions are cooler than tanks that contain concentrated solutions. Also, tanks that are relatively full in general have higher temperatures than tanks that are relatively empty. A notable exception is tank 241-AP-101, which contains $4224 \mathrm{~m}^{3}(1,116,000$ gal) of solution which is saturated in aluminum salts and some other compounds. Tank 241-AP-101 has an average temperature of $18.6^{\circ} \mathrm{C}\left(65.4^{\circ} \mathrm{F}\right)$. An evaluation was made of the effect of cooling this waste by approximately $5.6^{\circ} \mathrm{C}\left(10^{\circ} \mathrm{F}\right)$. It was determined that cooling this waste would result in the formation of solids, however the volume of solids in the solution remains much lower than 5 vol\%.

${ }^{1}$ ESP is a trademark of OLI Systems, Inc. 
HNF-2238

Revision 0

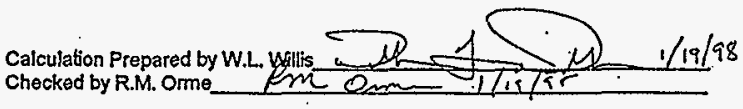

We Need to determine the flow characteristics for a transfer from Tank 241-AW-101 to Tank241-AP-1 J2. First the routing to be used is needed:

Starting at Tank 241-AW-101 Central Pump Pit 01A, the routing goes through line 3"SN $1-261-M 2$ to the 241-AW-A Valve Pit. IT IS IMPORTANT TO NOTE THAT THE PIPING CONNECTIONS IN THE 241-AW-A VALVE PIT ALLOWING CONNECTION OF LINE 3"SN-26i-M25 WITH 3 'SN-267-M25 IS NOT CURRENTLY IN PLACE. UPGRADES TO THE JUMPER CONFIGUIRATION IN THE 241-AW-A VAIVE PI ARE REQUIRED BEFORE THIS TRANSFER CAN BE MADE.

From the 241-AW-A Valve Pit, the routing goes through line $3^{m}$ SN-267-M25 to Tank 241-AW-102 Central Fump Pit 02A. Valve Pit 241-AP.

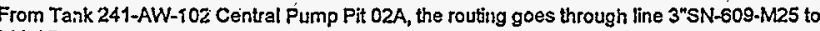

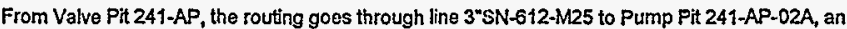
from the Finto the tank.

Assumptions:

The transter (up to 1,000,000 gallons) yill need to occur over a period of no more than five c'ays.

$$
Q:=\frac{1000000 \cdot \mathrm{gal}}{5 \cdot \text { day }}
$$

Or converting units

Flow := Q

$$
\text { Flow }=0.309 \cdot \mathrm{t}^{3} \cdot \sec ^{-1}
$$

The next step is to convert the flow rate to a velocity, first we need to calculate the cross section of . the piping. For Schedule 40 pipe the inside dimeter is 3.068 in

$\mathrm{D}:=3.068 \cdot \mathrm{in} \quad$ Area $:=\pi \cdot\left(\frac{\mathrm{D}}{2}\right)^{2} \quad$. Area $=0.051 \cdot \mathrm{n}^{2}$

The mean velocity in the pipe is then given by dividing the flow by the cross sectional area

$$
\text { vel }:=\frac{\mathrm{Q}}{\text { Area }} \quad \text { vel }=6.028 \cdot \mathrm{ft} \cdot \mathrm{vec}^{-1}
$$

Now we nead to cleternine the reynole's number in order to calculate the pressure losses in the pipe.

Reynolds number for flow through a pige is a function of the fluid density, mean velocity, pipe diameter, and fulid viscosity. Mean velocity and pipe diameter have already been defined, but we need to define fluid density and viscosity. Analysis has not been completed on the Waste in Tnak 241-AW-101, hiswever, analysis done on Tank 241-AN-105 supernate (which is assumed to contain waste which is similar to the waste in Tank 241-AW-101) shows the following for $50 \%$ dilution:
At $25 \operatorname{deg} C$
At $45 \operatorname{deg} \mathrm{C}$
At $65 \operatorname{deg} \mathrm{C}$
$\rho 25:=1.34 \cdot \frac{\mathrm{gm}}{\mathrm{mI}}$
$\rho 45:=1.32 \cdot \frac{\mathrm{gm}}{\mathrm{mL}}$
$\rho^{65}:=1.31 \cdot \frac{\mathrm{gm}}{\mathrm{mi}}$ 


$$
\mu 25:=6 \cdot 10^{-2} \text { poise } \quad \mu 45:=4 \cdot 10^{-2} \text { poise } \quad \mu 65:=2.5 \cdot 10^{-2} \text { poise }
$$

Now that the density and viscosity are defined the reynolds numbers can be calculated at the various temperatures. Reynolds number is defined as follows

$$
\operatorname{Re}:=\frac{\rho U D}{\mu} \text {. }
$$

For the temperatures listed above the reynolds numbers are as follorrs

$$
\begin{array}{ll}
\operatorname{Re} 25:=\frac{\rho 25 \cdot \text { vel.D }}{\mu 25} & \operatorname{Re} 25=3.197 \cdot 10^{4} \\
\operatorname{Re} 45:=\frac{\rho 45 \cdot \text { vel.D }}{\mu 45} & \operatorname{Re} 45=4.725 \cdot 10^{4} \\
\operatorname{Re} 65:=\frac{\rho 65 \cdot \text { vel } \cdot \mathrm{D}}{\mu 65} & \operatorname{Re} 65=7.502 \cdot 10^{4}
\end{array}
$$

From Hydraulic Institute "Engineering Data Book" 2nd Edition, The friction factors for these reynolds numbers are:

$$
\mathrm{f} 25:=0.02525 \quad \mathrm{f} 45:=0.02350 \quad \text { f65 }:=0.02170
$$

The formula for $\triangle P$ in a pipe is given by

$$
\text { hf }:=f \cdot\left(\frac{I}{D}\right) \frac{V^{2}}{2 \cdot g}
$$

Where $h f$ is the total head loss in $f t$ of water, $f$ is the friction factor, $L$ is the pipe length, $D$ is the pipe diameter, $V$ is the mean fluid velocity, and $g$ is the acceleration due to gravity. By rearranging, we arrive at the pressure drop per unit length: $h f /=(f / D) \times V / 2 g$. The pressure drops per unit langth for the 3 temperatures are isted trelow:

$$
\begin{array}{lll}
\text { hfl } 25:=\left(\frac{f 25}{D}\right) \cdot \frac{\text { vel }^{2}}{2 \cdot g} & \text { hff } 45:=\left(\frac{f 45}{D}\right) \cdot \frac{\text { vel }^{2}}{2 \cdot g} & \text { hfl } 65:=\left(\frac{f 65}{D}\right) \cdot \frac{\text { eel }^{2}}{2 \cdot g} \\
\text { hfL } 25=0.056 & \text { hfi } A 5=0.052 & \text { hfL } 65=0.048
\end{array}
$$


HNF-2238

Revision 0

Now we need to determine the lengths of pipe and the eguivalent lengths of the fittings in the route. the first leg in the route is from the tank to the outlet from the 01A Central Pump Pit. The configuration of this leg is included as figure 2 . The leg contains the following components

$$
\begin{aligned}
& \text { linea }:=12 \cdot f 3^{m} \text { schedule } 40 \text { pipe } \\
& \text { conecta }:=23^{\prime \prime} \text { PUREX type connectors } \\
& \text { twvta }:=13^{\prime \prime} 3 \text { way ball valve (fiow through run) } \\
& \lg 90 \mathrm{a}:=23^{n} \text { long radius } 90^{\circ} \text { elbow's }
\end{aligned}
$$

The second section of the route is the $3^{n}$ SN-261-M25 Lne which contains the following components

lineb :=96. $\mathrm{ft}^{\prime \prime}$ schedule $40^{\circ}$ pipe

$\lg 90 \mathrm{~b}:=33^{\prime \prime}$ long radius $90^{\circ}$ elbows

The third section of the route is in the 241-AW-A Valve Pit (it is imporiant to note that the following components do not exist. Work to design fabrica:e and install these components is part of the W-454 Project.). The 241-AW-A Valve Pit is assumed to contain the following components:

linec := 12, ft $3^{\text {" schedule }} 40$ pipe

conectc : $=33^{\prime \prime}$ PUREX type connectors

twvtc: : 13 " 3 way ball valve (flow through run)

twvbc := $33^{n} 3$ way ball valve (flow through branch)

blvlve := 23 " bail values

$\lg 90 \mathrm{c}:=13^{n}$ long radius $90^{\circ}$ elbow

The forth section of the route is the $3^{\prime \prime S N-267-M 25 ~ L i n e ~ w h i c h ~ c o n t a i n s ~ t h e ~ f o l l o w i n g ~ c o m p o n e n t s ~}$

lined := $105 \cdot \mathrm{ft}^{*}$ schedule 40 pipe

$\lg 90 \mathrm{~d}:=53^{\prime \prime}$ long radius $90^{\circ}$ elbows

The fifth section of the route is in the Tank 241-AW- $1 \mathrm{C} 2,02 \mathrm{~A}$ Central Pump Pit, and includes the following components

linee $:=10 \cdot \mathrm{ft}^{3}$ schedule 40 pipe

conecte := 23 "PUREX type connectors

twrte - $13^{n} 3$ way ball valves (flow through run) 
HNF-2238

Revision 0

twvte . $13^{*} 3$ way ball valves (llow through run)

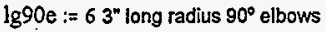

The sixth section of the route is the 3"SN-609-M25 Line which contains the following components

linef : $=725 \cdot \mathrm{At} 3^{\prime \prime}$ schedule 40 pipe

$\lg 90 \mathrm{f}:=133^{\prime \prime}$ long radius $90^{\circ}$ elbows

The seventh secticn of the routg is in the 241.AP Valve Pit, and includes the following components

lineg := 35-ft $3^{\text {" schedule }} 40$ pipe

conectg : $53^{\prime \prime}$ PUREX type conrecters

twvtg : $=13^{\text {" }} 3$ way ball valves (flow through run)

twvbg $\square 23^{\text {" }} 3$ way ball valves (flow through branch)

bivlvg $:=33^{\text {" ball valves }}$

$\lg 90 \mathrm{gg}:=113^{\prime \prime}$ long radius $90^{\circ}$ elbows

st $90 \mathrm{~g}:=13$ " standard radius $90^{\circ}$ elbow

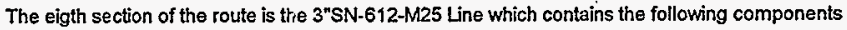

lineh := 410. ft $3^{\prime \prime}$ schedule 40 pipe

$\lg 90 \mathrm{~h}:=8$ long radius $90^{\circ}$ elbows

The ninth and last section of the route is in the 241-AP-02A Cenfral Fump Pit, and includes the following components

linei := 45- $\mathrm{ft} 3^{\prime \prime}$ schedule 40 pipe

conscti := $53^{\text {n }}$ PUREX type connectors

twvti := $33^{n} 3$ way ball valve (flow throuth run)

lg90i : $:=73^{n}$ lony radius $90^{\circ}$ elbows

st90i := 13 "siandard radius $90^{\circ}$ elbow

st $45 i:=13^{\prime \prime}$ standard radius $45^{\circ}$ elbow

The tntal fine length is given by the sum of all the line segments

linetot $:=$ lirea + lineb + linec + lined + linee + linef $\div$ lineg + lineh + linei

linetot $=1.45 \cdot 10^{3} \cdot \mathrm{ft}$

Now to determine the equivalent length of the fittings, the number of each type fitting is multiplied by the equivalent length in pipe diameters $(I / D)$ from Crane Technical Paper \# 410 "Flow of Fluids through Valves Fittings, and Pipe".

First for the PUREX type connectors (assumed to have equivalent pressure drcp as in an angle valve),

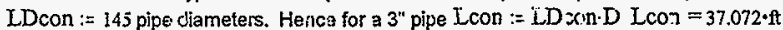


HNF-2238

Revision 0

For a ball valve with flow through run the equivalent length in pipe diameters is

LDtwvt := 44 pipe diameters. Hence for $3^{\prime \prime}$ pipe Ltwvt := LDtwvt-DLtwvt $=11.249 \cdot \mathrm{ft}$

For a ball valve with flow through branch the equivalent length in pipe diameters is

LDtwvb := 140 pipe diameters. Hence for 3" pipe Itwwb := LDtwvb.D Ltwwb $=35.793 \cdot \mathrm{f}$

For a ball valve the equivalent length in pipe diameters

LDblvlv : $=18$ pipe diameters. Hence for $3^{\prime \prime}$ pipe Lblvlv := LDblvlv.DLblvlv $=4.602 \cdot \mathrm{ft}$

For long radius $90^{\circ}$ elbow, the equivalent length is $\mathrm{LDlg} 90:=20$ pipe diameters. Hence for $3^{n}$ pipe, $\operatorname{Lig} 90:=\operatorname{LD} \lg 90 \cdot \mathrm{D}$ Llg90 $=5.113 \cdot \mathrm{ft}$

For a standard radius $90^{\circ}$ elbow, the equivalent length is $L D$ st $90:=30$ pipe diameters. Hence for $3^{\prime \prime}$ pipe, Lst90 := LDst90.D Lst90 $=7.67 \cdot$ ft

For standard radius $45^{\circ}$ elbows, the equivalent length is IDst $45:=16$ pipe diameters. Hence for $3^{n}$ pipe, Lst45 := LDst45.D Lst45 $=4.091 \cdot$ ft

Now, we calculate the eqivalent length of the fittings. First for the PUREX connectors

ELcon $:=($ conecta + conectc + conecte + conectg + conecti $) \cdot$ Lcon

$E L \operatorname{con}=630.218 \cdot \mathrm{ft}^{\circ}$

Next the three way ball valves with flow through run

ELtwvt : a (twvta + twvtc + twrte + twvtg + twvti) $\cdot$ Ltwrt

ELtwvt $=78.745 \cdot \mathrm{ft}$

Next the three way ball valves with flow through branch

ELtwvb : $:($ twvbc + twwbg) $\cdot$ Ltwvb

Eltwovb $=178.967 \cdot$ ft

Next the loing radius $90^{\circ}$ elbows

ELlg $90:=(\lg 90 \mathrm{a}+\lg 90 \mathrm{~b}+\lg 90 \mathrm{c}+\lg 90 \mathrm{~d}+\lg 90 \mathrm{e}+\lg 90 \mathrm{f}+\lg 90 \mathrm{~g}+\lg 90 \mathrm{~h}+\lg 90 \mathrm{i}) \cdot \operatorname{Ll} 390$

$\mathrm{ELIg} 90=286.347 \cdot \mathrm{ft}$

Next the standard radius $90^{\circ}$ slbows

ELst $90:=($ st $90 \mathrm{~g}+$ st90i) $\cdot$ Lst $90 \quad$ ELs $\$ 90=15.34 \cdot$ ft 
HNF-2238

\section{Revision 0}

Finally, the standard radius 450 elbow

ELst $45:=($ st45i) $\cdot$ Lst45 ELst $45=4.091 \cdot \mathrm{ft}$

Now we can calculate the total equivalent length of all the fittings

ELftot := ELcon + ELtwvt + ELtwvb + ELlg90 + ELst90 + ELst45

ELftot $=1.194 \cdot 10^{3} \cdot \mathrm{ft}$

Now the total effective pipe length is the sum of the pipe length and the equivalent lergth of all the fittings

ELtot : $=$ linetot + ELftot $+40 \cdot f t$

ELtot $=2.684 \cdot 10^{3} \cdot \mathrm{ft}$

The additional $40 \mathrm{ft}$ is added to account for the length of the discliarge pjpe in the tank. The total estimated head losses for each of the three temperatures can now be calculated
Loss $25:=$ hfl $25 \cdot$ ELtot
$\operatorname{Loss} 25=149.651 \cdot \mathrm{ft}$
Loss $45:=$ hef 45 .ELtot
$\operatorname{Loss} 45=139.279 \cdot \frac{A t}{2 t}$
Loss $65:=$ hfll 65 -ELtot
$\operatorname{Loss} 65=128.611 \cdot f t$

In addition to the pressure drop, there is a $41 \mathrm{ft}$ gain in the transfer from the bottom Tank 241-AW-\{01 to the top of Tank 241-AP-102. Therefore, assuming a temperature of $25^{\circ} \mathrm{C}$, if the fluid being transferrred were water, the pump would need cieliver a minimum head of approximately 191 fi. Since the density of the solution is 1.34 , the minimum dischargo head required is $255 \mathrm{ft}$ or $111 \mathrm{psi}$.

$$
-=
$$


HNF-2238

Revision 0
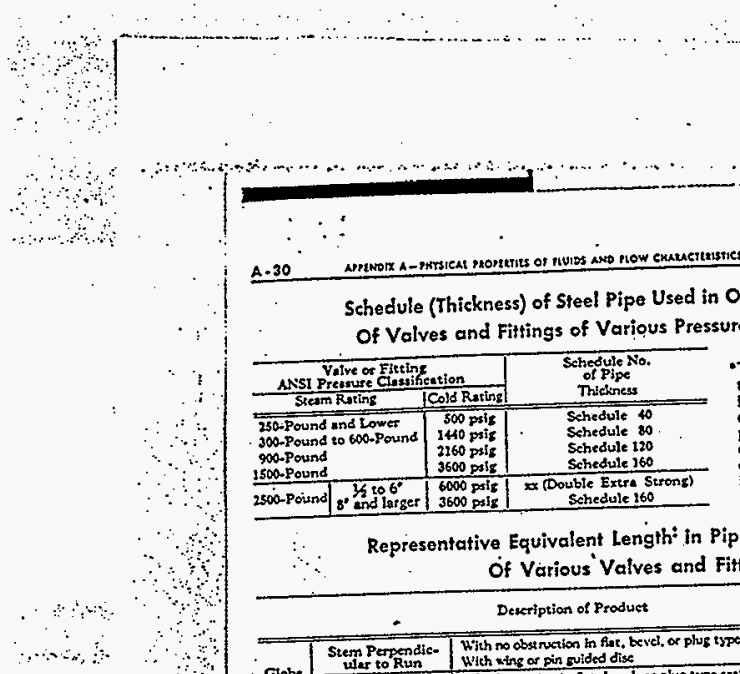
trasily selected anly for the purpose of identifying the various pressure elastes of valves and fittings with specife pipe dimensions for the interpretation of pew test data; they should not be construed 23 a recommendation for installation purposes.

2500-Poind $\left[8^{\prime}\right.$ and leiger $\mid 3600$ paig $\mid$ Schedule 160.

Diameters $(L / D)$

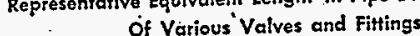

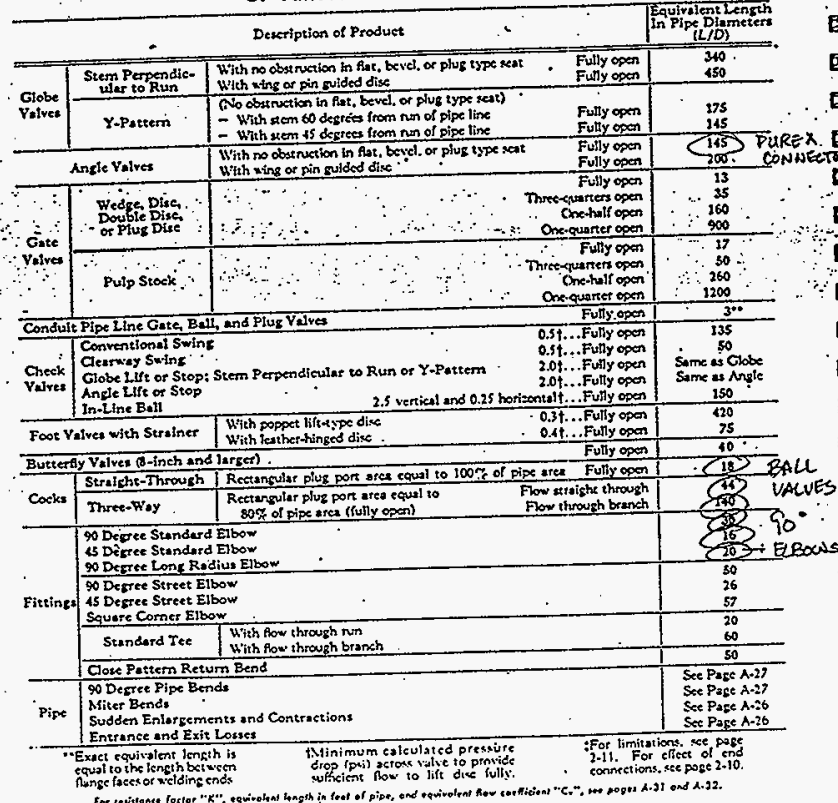




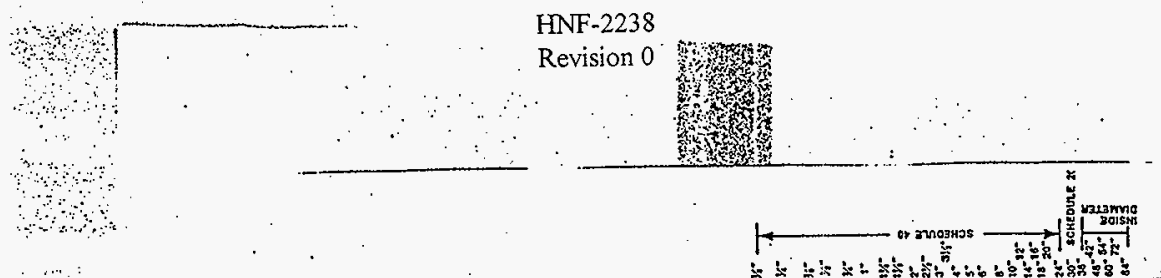

HNF-2238

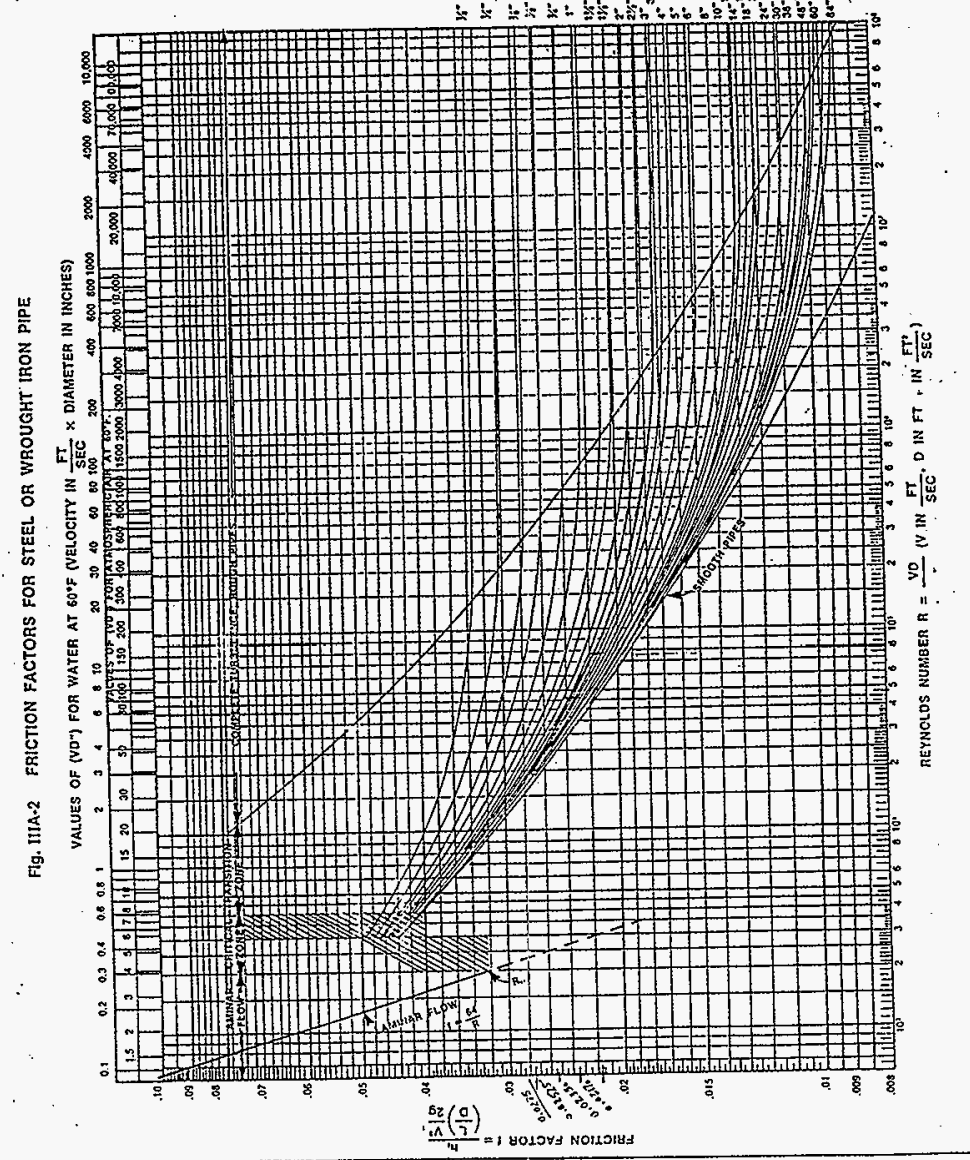

กำ Mำ

D-12 


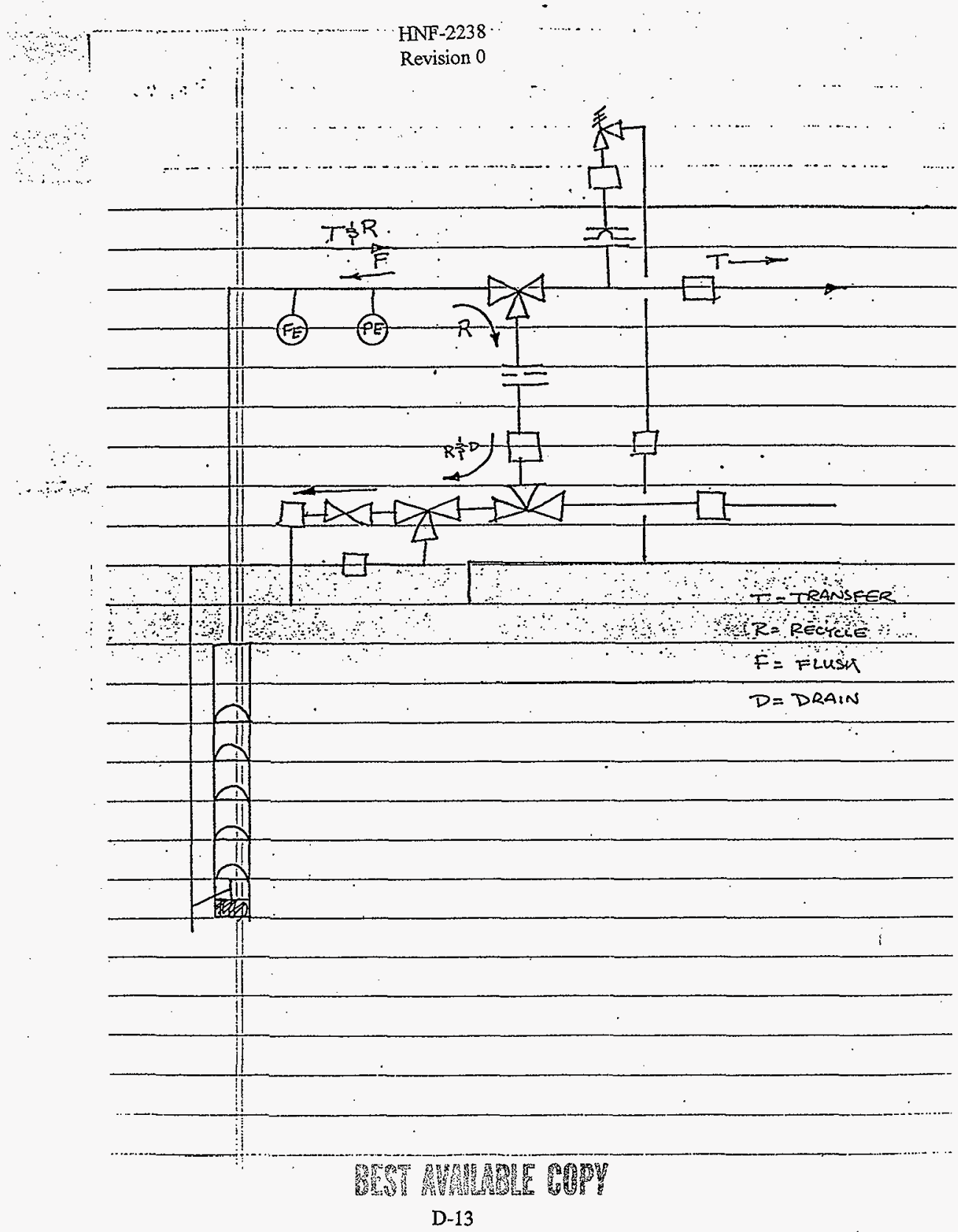


For 2' Pipe Lengths of feet

\begin{tabular}{|c|c|c|c|c|c|c|c|c|c|c|}
\hline Flow & & & & & & & & & & \\
\hline Rate & 1000 & 2000 & 3000 & 4000 & 5000 & 60000 & 7000 & 8000 & 9000 & 10000 \\
\hline (Gpm) & Total Pres & ure drops ( & eet of wate & & & & . & & & \\
\hline 0 & 0 & 0 & . 0 & 0 & 0 & 0 & 0 & 0 & 0 & 0 \\
\hline 5 & & $\cdot$ & & & & & & & . & \\
\hline 10 & 3.74 & 7.47 & $\$ 1.21$ & 14.95 & 18.69 & 224.22 & 26.16 & 29.90 & 33.63 & 37.37 \\
\hline 15 & 7.75 & 15.50 & 23.25 & 31.00 & 38.75 & 465.05 & 54.26 & 62.01 & 69.76 & 77.61 \\
\hline 20 & 12.99 & 25.97 & 38.96 & 51.95 & 64.94 & 779.24 & 90.91 & 103.90 & 116.89 & 129.87 \\
\hline 25 & 19.42 & 38.85 & 58.27 & 77.69 & 97.12 & 1165.40 & 135.96 & 155.39 & 174.81 & 194.23 \\
\hline 30 & 27.22 & 54.44 & 81.66 & 108.88 & 136.10 & 1633.22 & 190.54 & 217.76 & 244.98 & . 272.20 \\
\hline 35 & 36.45 & 72.89 & 109.34 & 145.79 & 182.24 & 2186.85 & 255.13 & 291.58 & 328.03 & 364.47 \\
\hline 40 & 46.83 & 93.66 & 140.48 & 187.31 & 234.14 & 2809.69 & 327.80 & 374.62 & 421.45 & 468.28 \\
\hline 45 & 57.90 & 115.80 & 973.70 & 231.60 & 289.50 & 3474,04 & 405.31 & 463.21 & 521.11 & 579.01 \\
\hline 50 & 70.36 & 140.71 & 211.07 & 281.43 & 351.79 & 4221.45 & 492.50 & 562.86 & 633.22 & 703.57 \\
\hline 55 & & & & & & & & & & \\
\hline 60 & 99.42 & $198: 85^{\circ}$ & 298.27 & 397.69 & 497.12 & .5965 .39 & 695.96 & $795.39^{\circ}$ & 894.81 & 894.23 \\
\hline 65 & & & & & & & & & & \\
\hline 70. & 132.87 & 265.74 & 398.62 & 531.49 & 664,36 & 7972.31 & 930.10 & 1062.97 & 1195.85 & 1328.72 \\
\hline 75 & & & & & 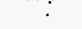 & & & & & \\
\hline 80 & 172.09 & 344.17 & 516.26 & 688.35 & 860.44 & 10325.25 & 1204.61 & 1376.70 & 1548.79 & 1720.87 \\
\hline 85 & & & & & & & & & & \\
\hline 90 & 214.53 & 429.07 & 643.60 & 858.13 & 1072.66 & 12871.96 & 1501.73 & 1716.26 & 1930.79 & 2145.33 \\
\hline 95 & . & & & . & & $\ddots$ & & & & \\
\hline 100 & 262.98 & 525.95 & 788.93 & 1051.80 & 1314.88 & 15778.53 & 5840,83 & 2103.80 & 2366.78 & 2629.76 \\
\hline $105^{\circ}$ & & & retes & & & & & & & \\
\hline 110 & & & & & & . & & & & \\
\hline 115 & & ' & $\cdot$ & & & & & • & & \\
\hline 120 & & & & & & & & & & \\
\hline 125 & 406.00 & 811.99 & 1217.99 & 1623.99 & 2029.99 & 24359.84 & 2841.98 & 3247.98 & 3653.98 & 4059.97 \\
\hline 130 & & - & & & & & & & & 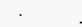 \\
\hline 135 & & & . & & & & & & & \\
\hline 140 & & & $\therefore$ & & & & & & & \\
\hline 145 & & & & & & & & & & \\
\hline 150 & 579.01 & 1158.01 & 1737.02 & 2316.03 & 2895.04 & 34740.45 & 4053.05 & 4632.06 & 5211.07 & 5790.07 \\
\hline 155 & & & & & & & & & & \\
\hline 160 & & & & & & & & & & \\
\hline 965 & & & & & & & & & & \\
\hline . 170 & & & . & & & & & & & \\
\hline . $\quad 175$ & 772.78 & 1545.56 & 2318.34 & 3091,12 & 3863.89 & 46366.73 & 5409.45 & 6182.23 & 6955.01 & 7727.79 \\
\hline 180 & & & & & & & & & . & \\
\hline 185 & & & & & & & & & & \\
\hline 190 & & & & & & & & & & \\
\hline 195 & & & & & & & & & & \\
\hline 200 & 996.54 & 1993.08 & 2989.62 & 3986.16 & 4982.69 & 59792.33 & 6975.77 & 7972.31 & 8968.85 & 9965.39 \\
\hline 205 & & & & & & & & & & \\
\hline 210 & & & . & & & & & & & \\
\hline 215 & & & & & & & & & & \\
\hline 220 & & & & & & & & & & \\
\hline 225 & & & & & & & & & & \\
\hline
\end{tabular}


HNF-2238

Revisiöñ $0^{\circ}$

For 3" Pipo Longths of.

foot

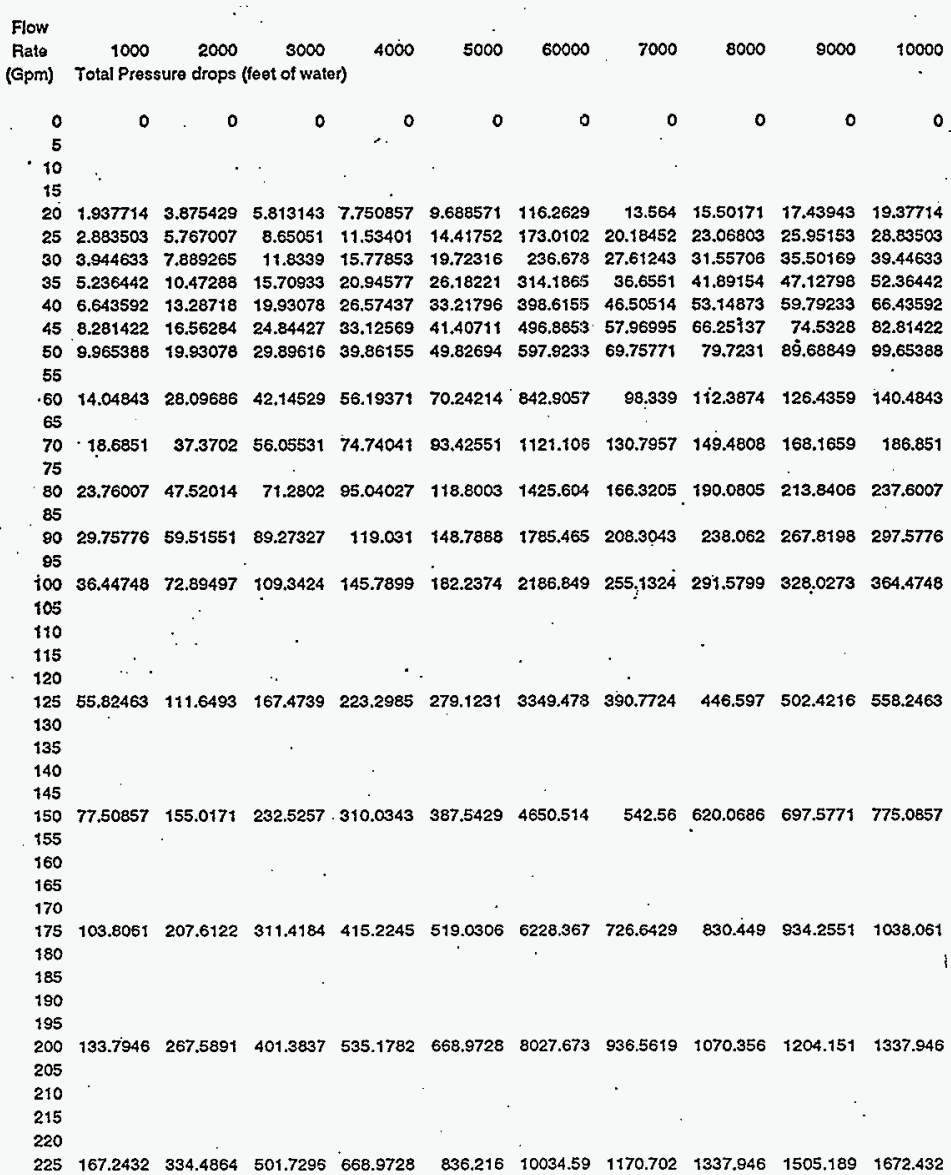


HNF-2238

Revision 0

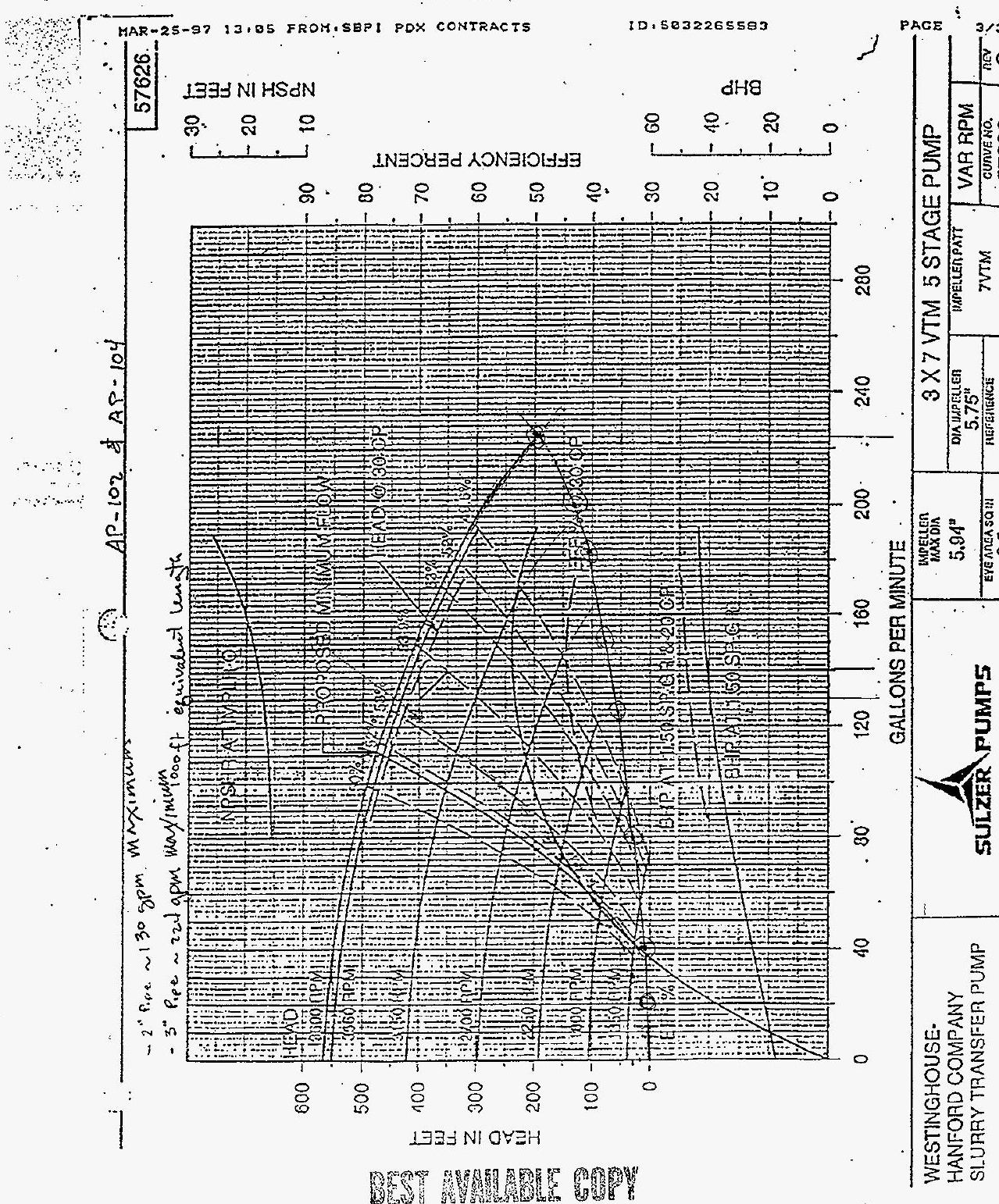




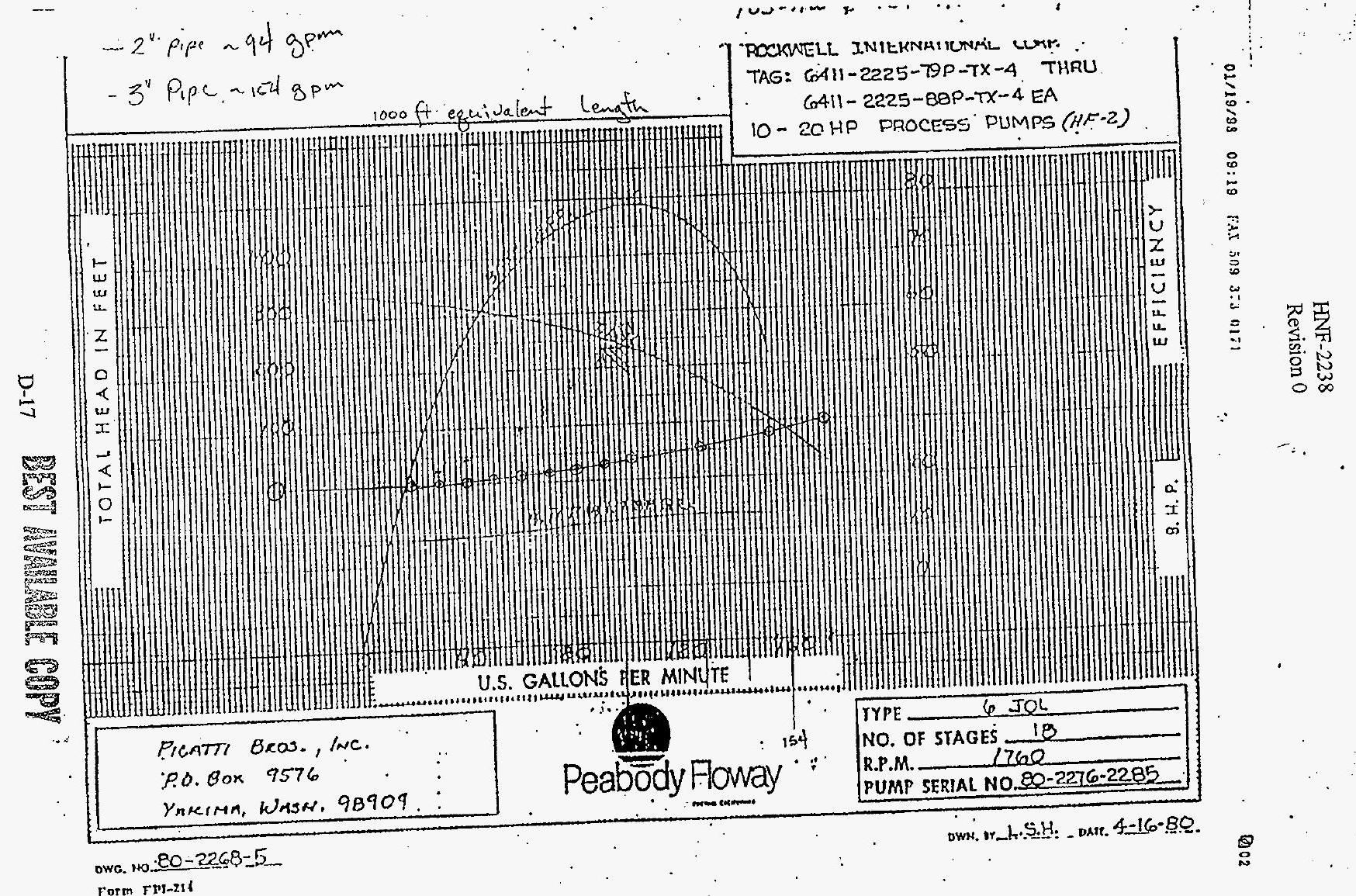


HNF-2238

Revision 0

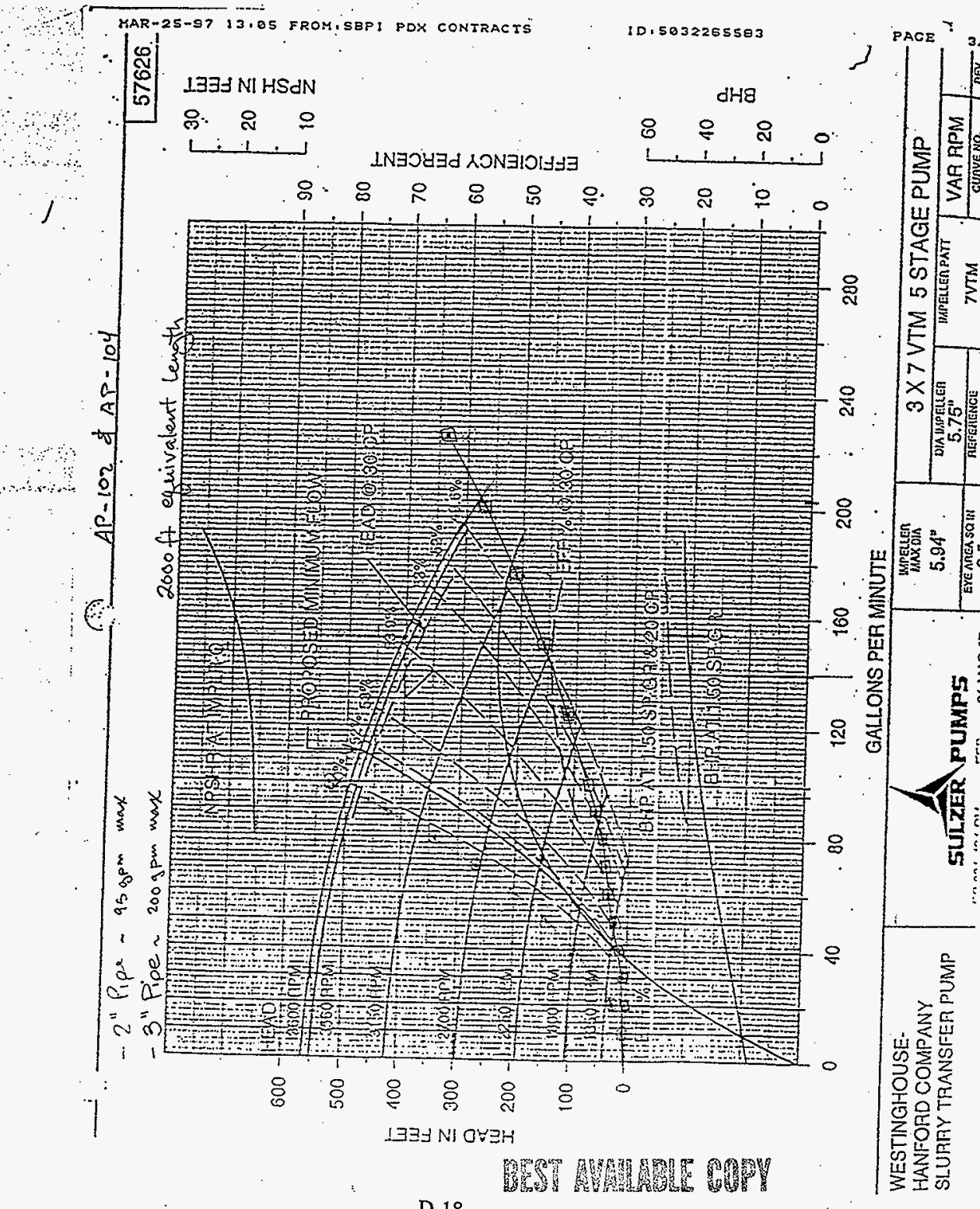


HNF-2238

Revision 0

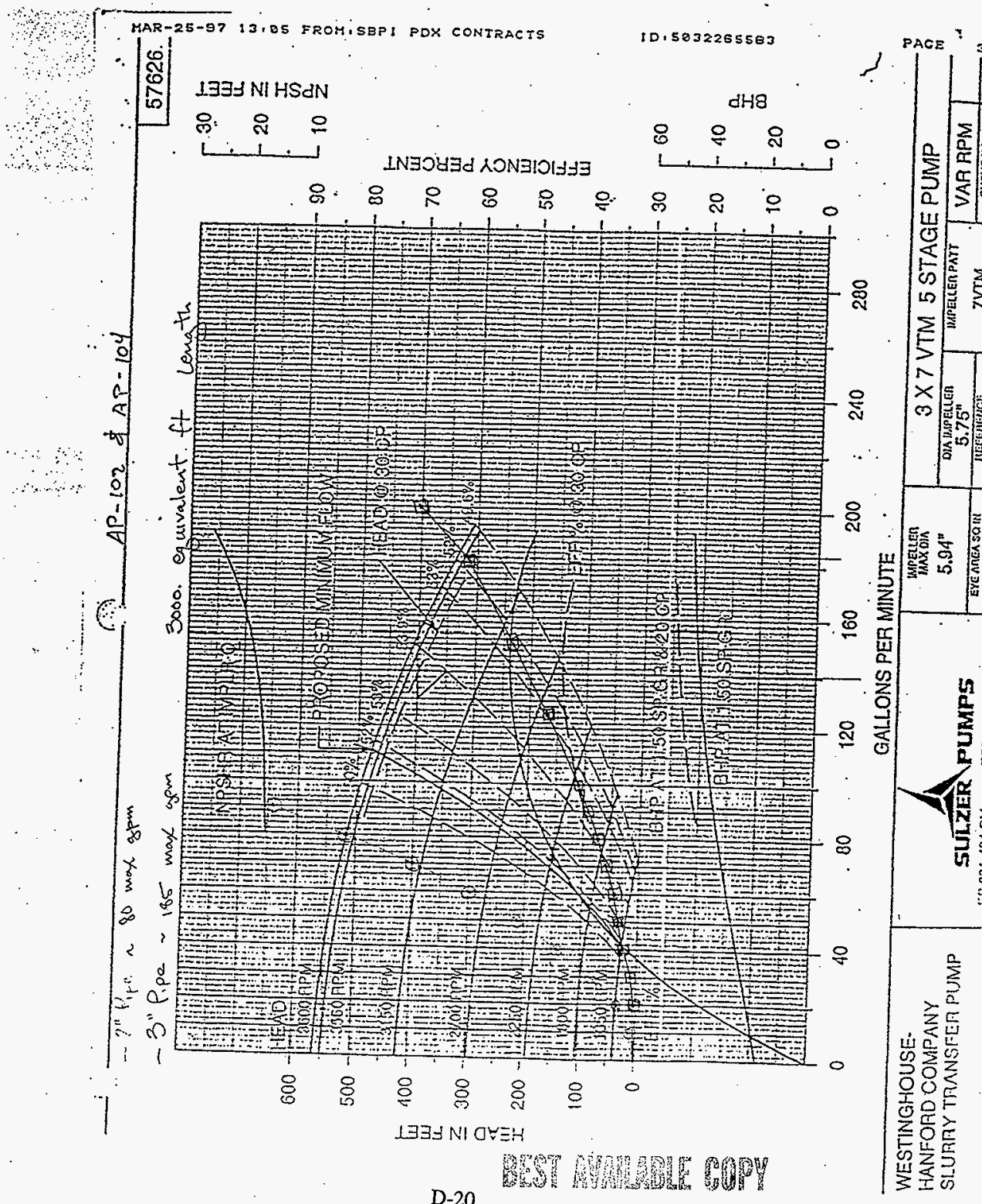


HNF-2238

Revision 0

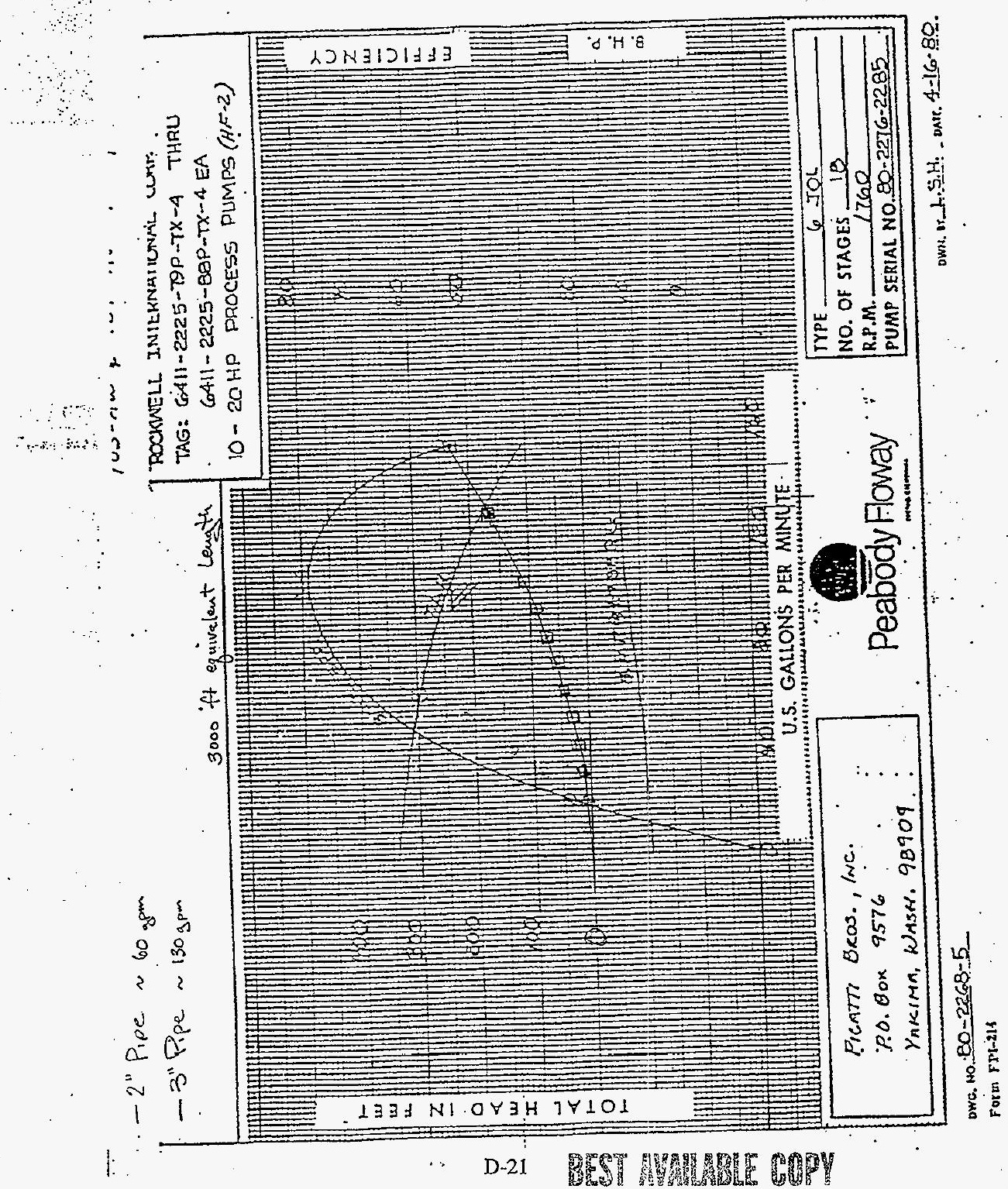


HNF-2238

Revision 0

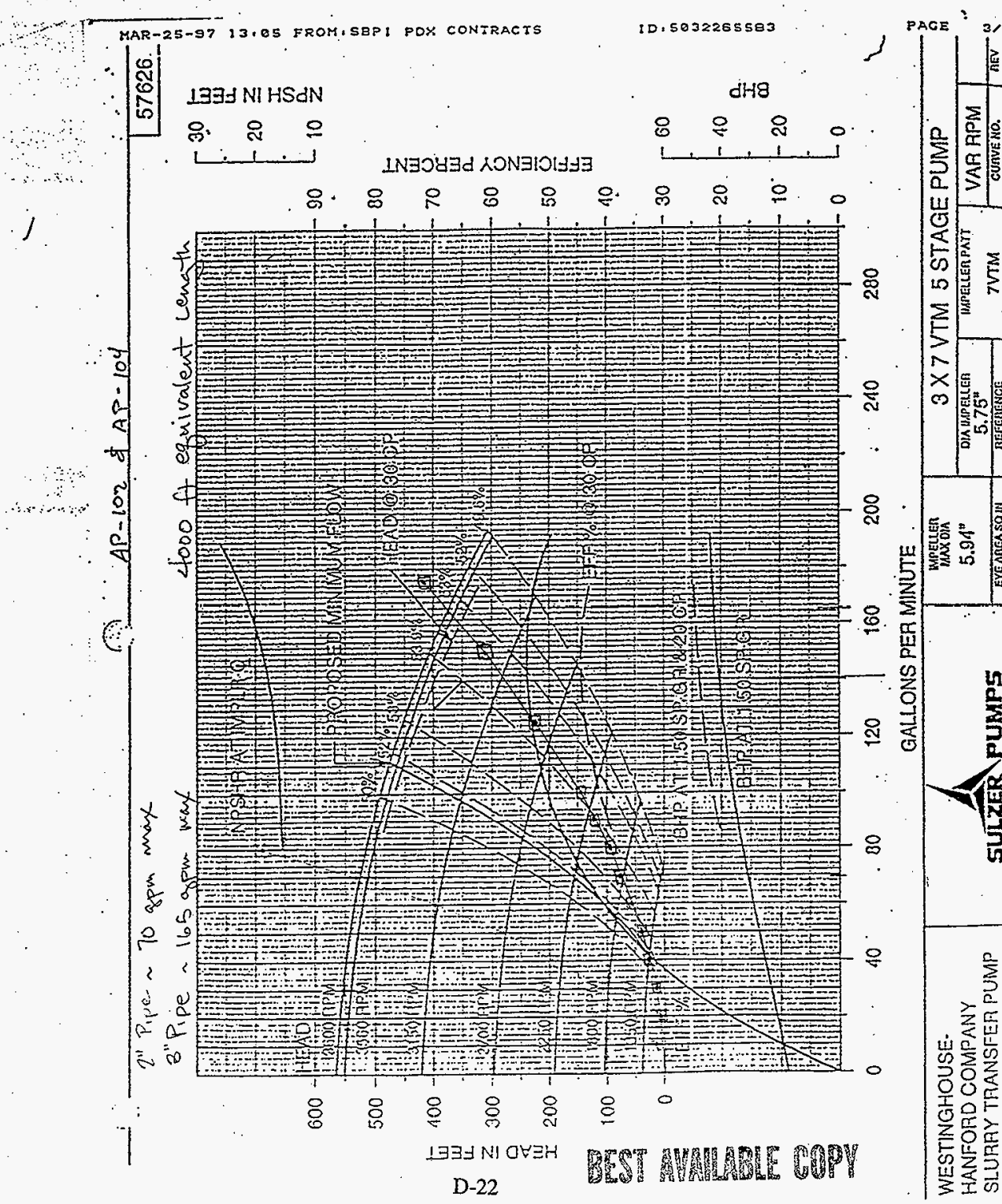




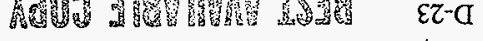

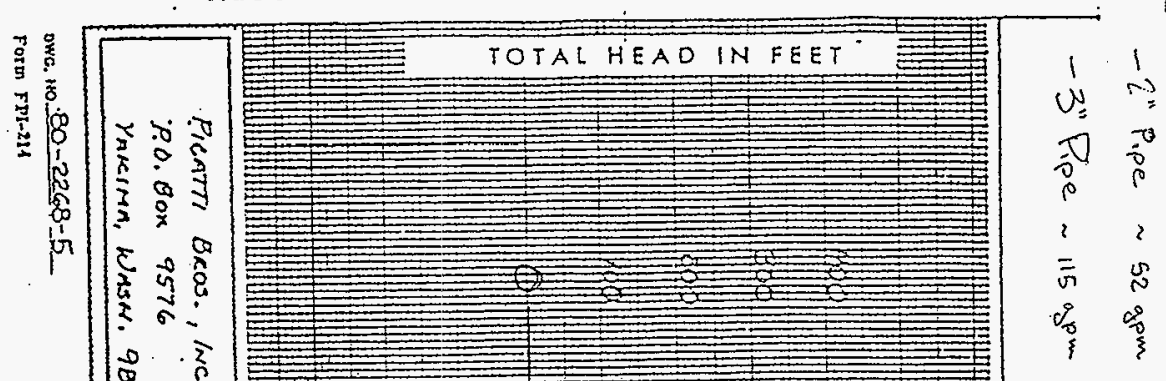

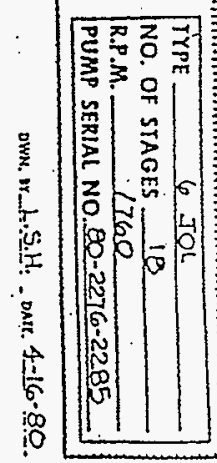

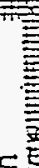

in

हn
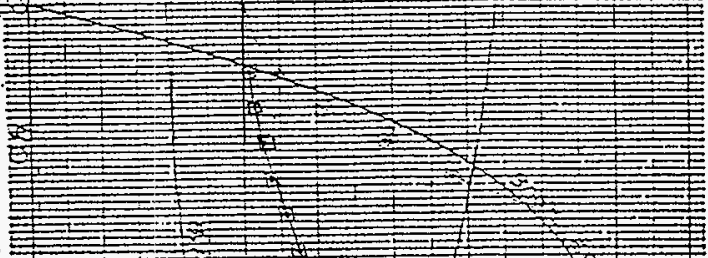

둥

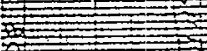

垌

要

ב

in

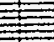

$\square$

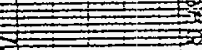

$+2$
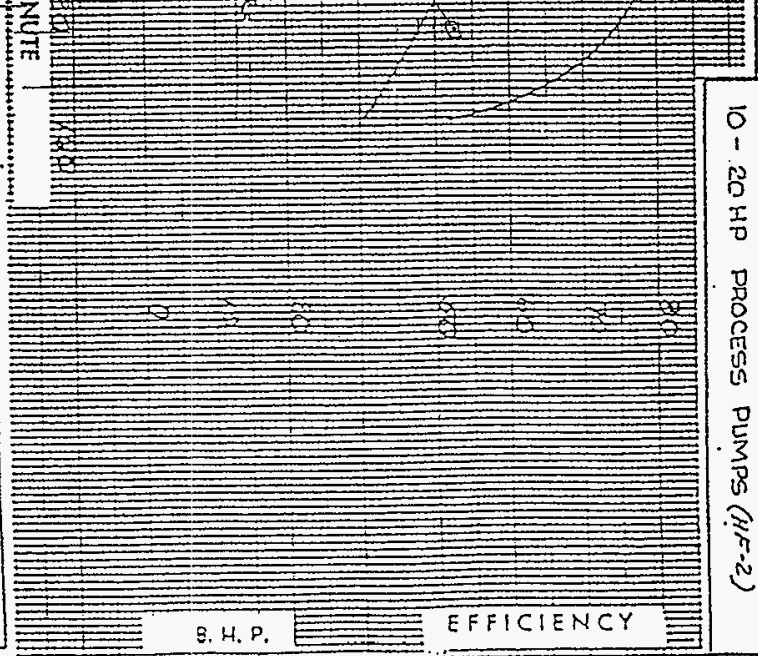

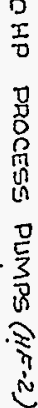

б: 零

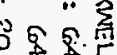

$\pm 三$

N

N N 2

N

1 i

0 d

0 च :

1 .

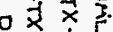

11

$8 \pi$

采

至

心

.

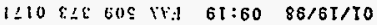


HNF-2238

Revision 0

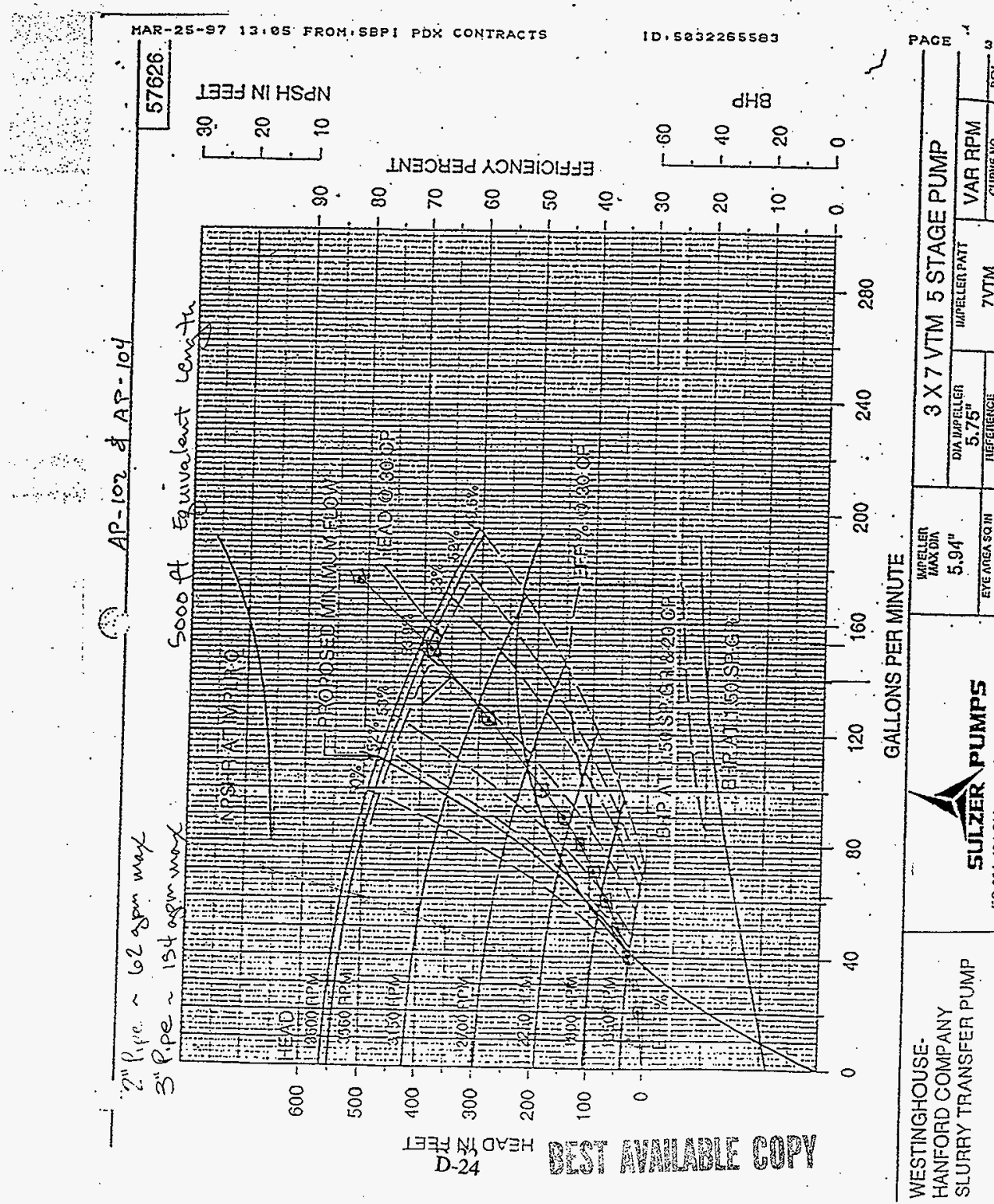


HNF-2238

Revision 0

$01 / 19 / 98$ 09:19 1:A1 505 373 0171

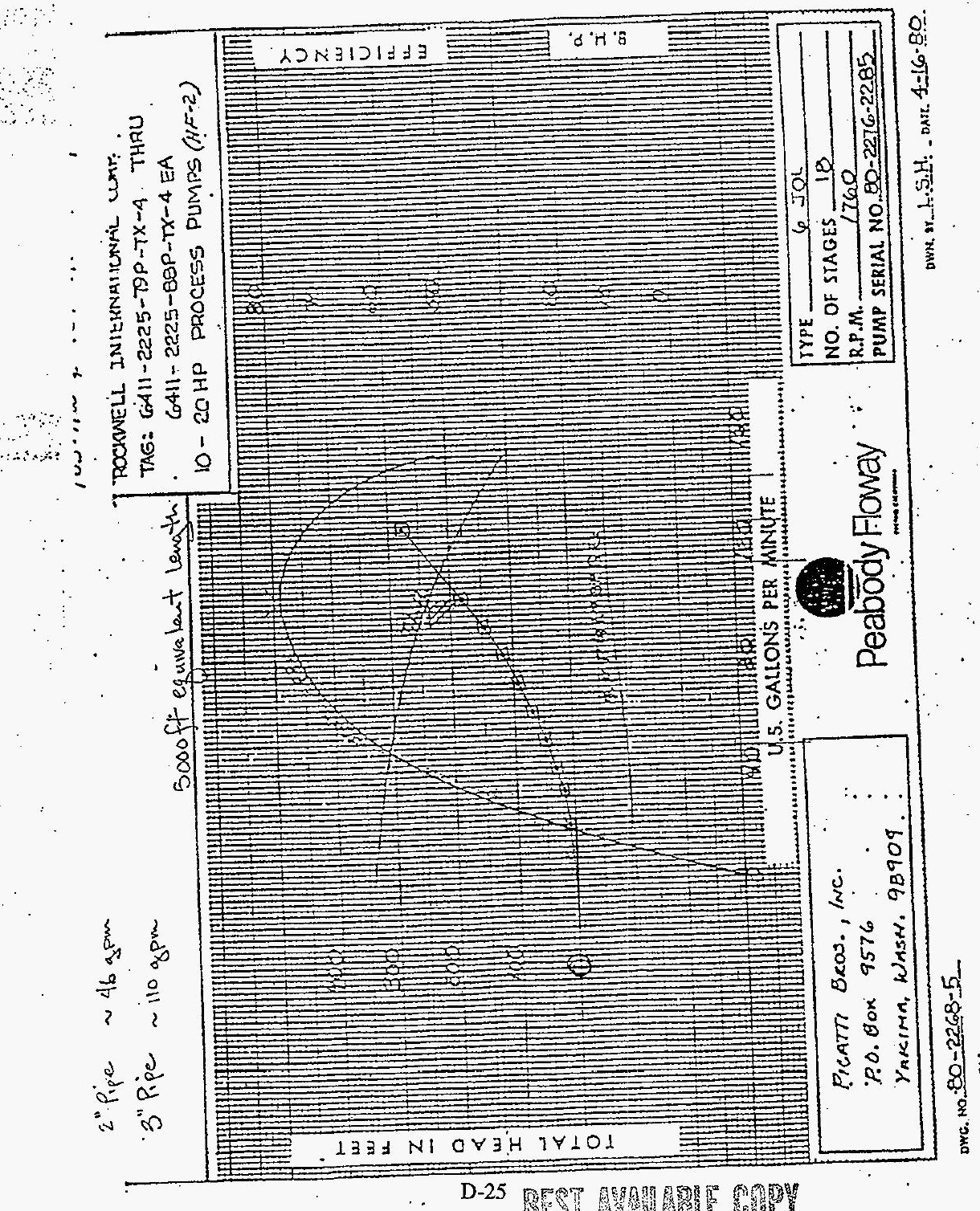

- 
HNF-2238

Revision 0

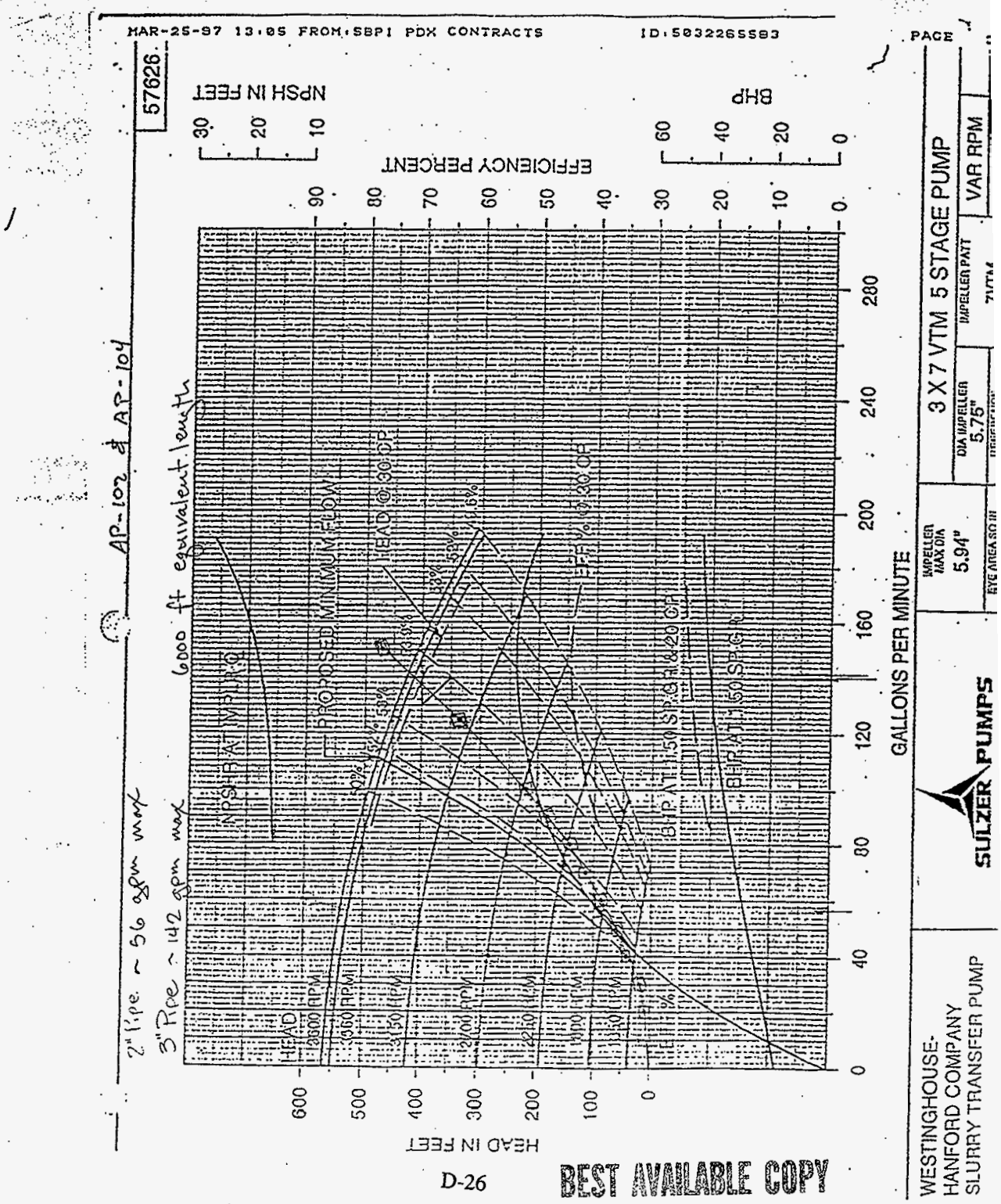


HNF-2238

Revision 0

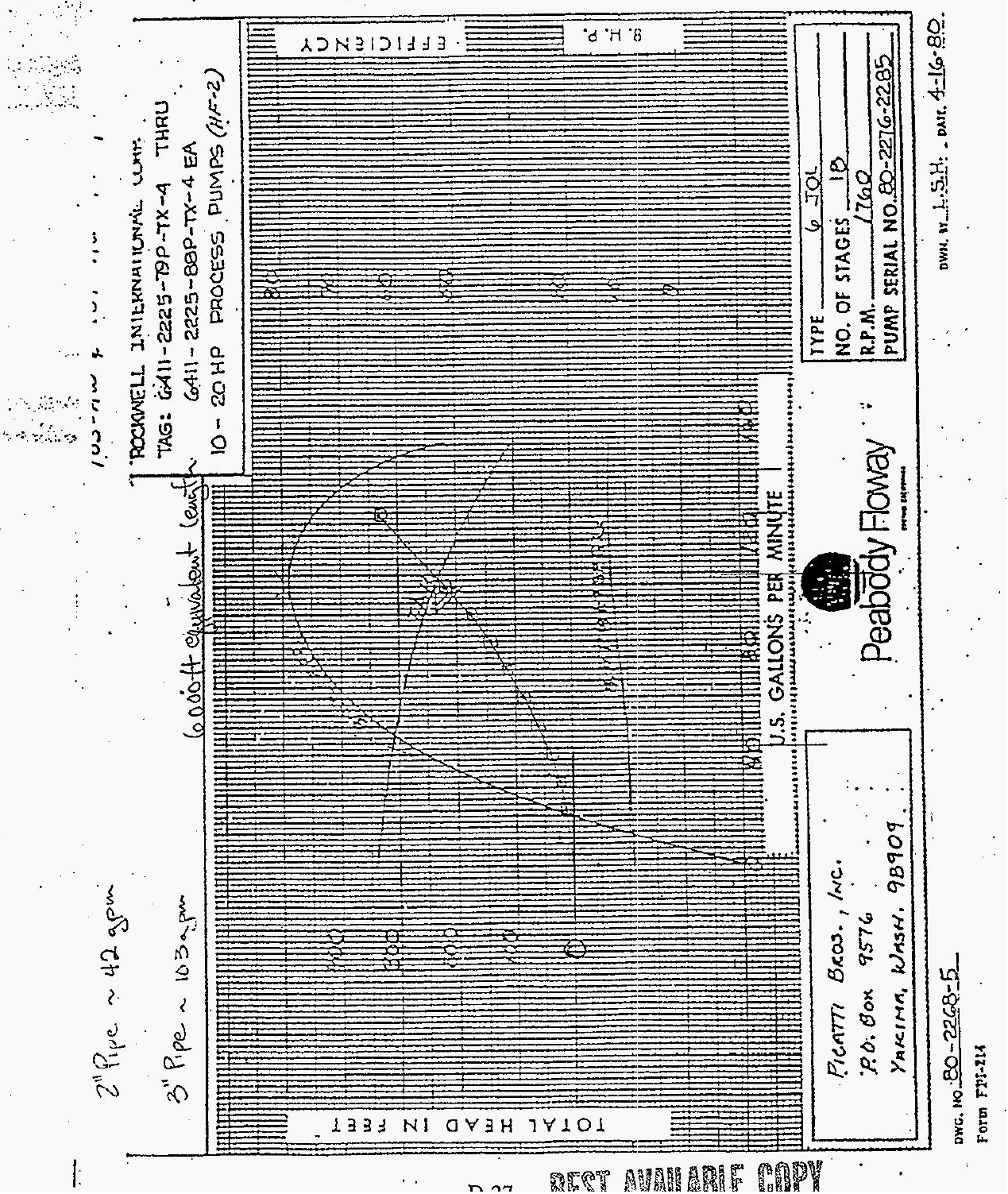


HNF-2238

Revision 0

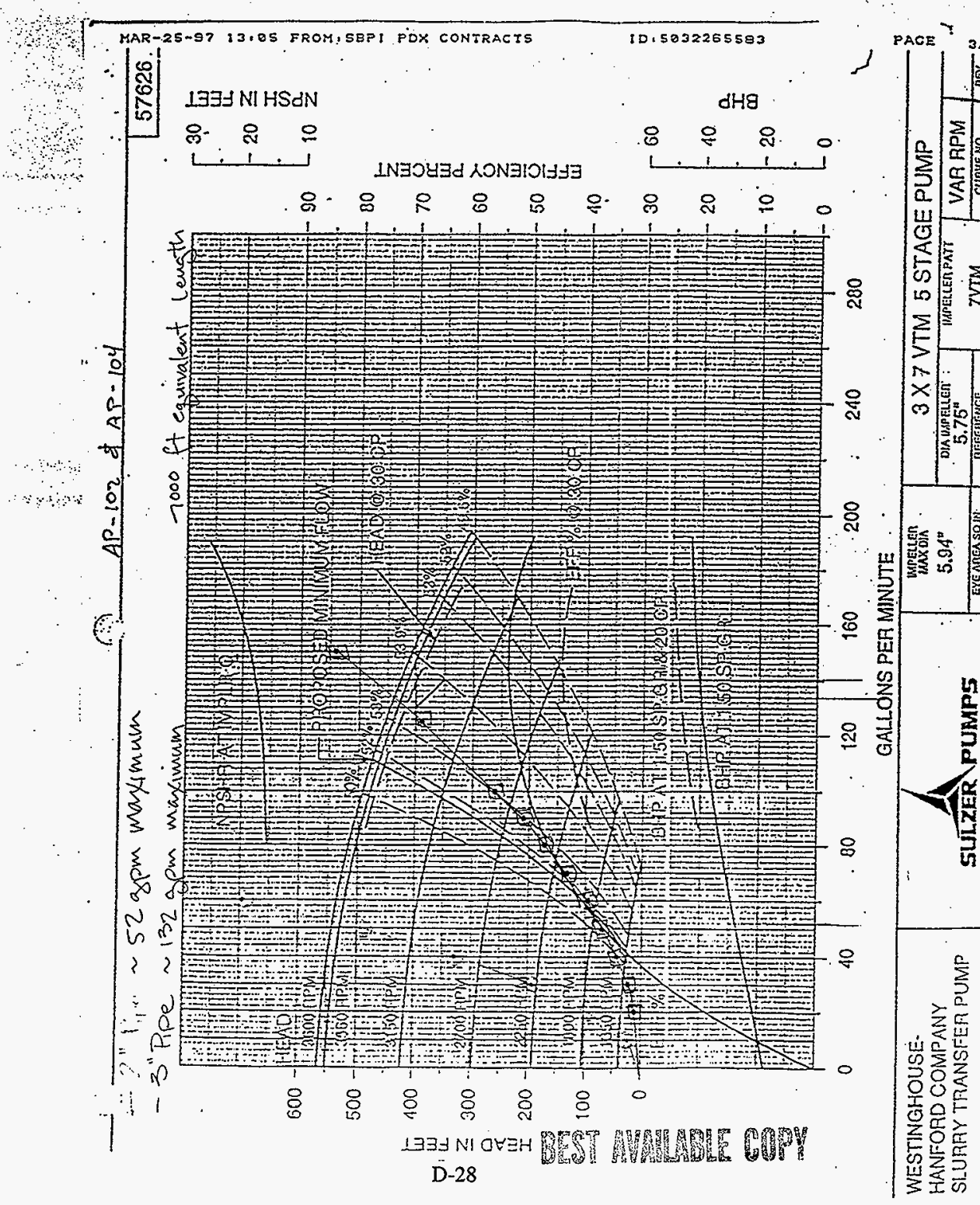




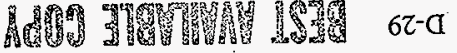

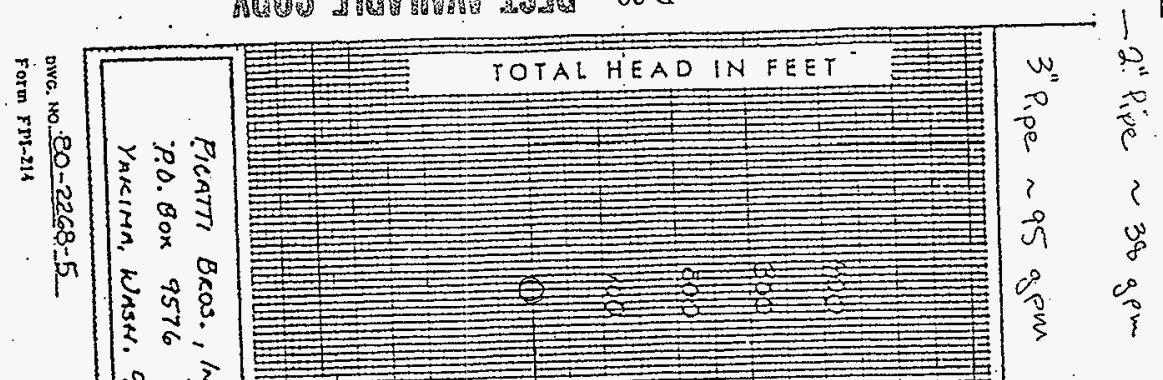

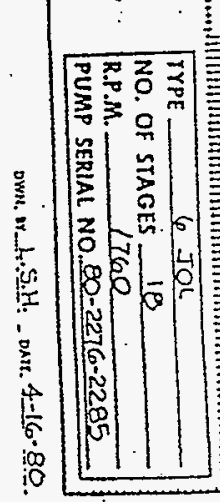

营

$\underbrace{\frac{1}{2}}_{\therefore}$

200

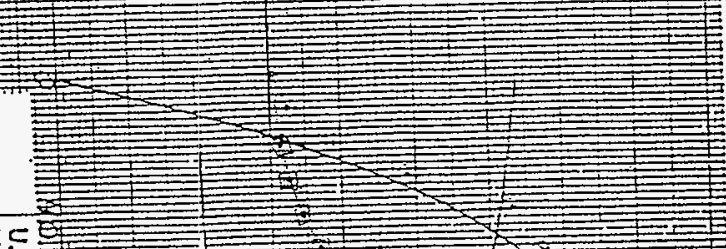

$\frac{1}{8}$

$\lim _{Q}$

$>210101010$
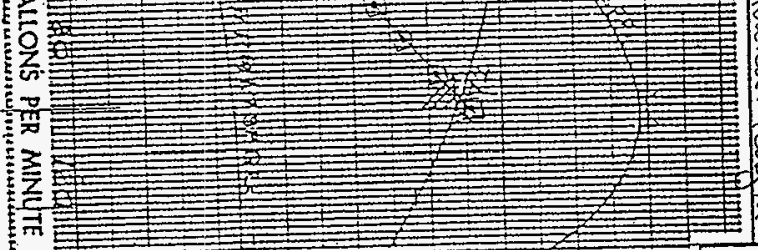
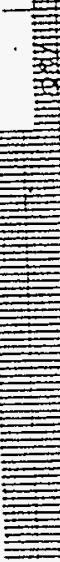

.
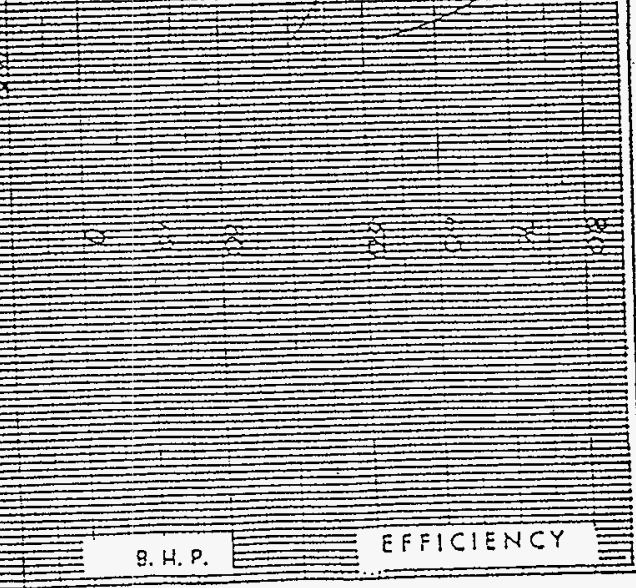

Q. H.P.

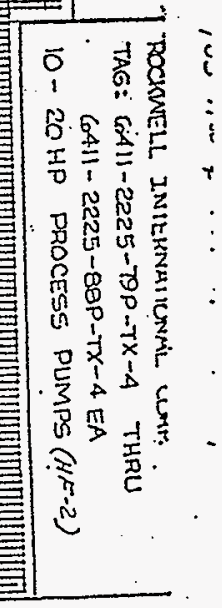

1:10 E:E :05 IV: $67: 60$ 86/6T/TO 


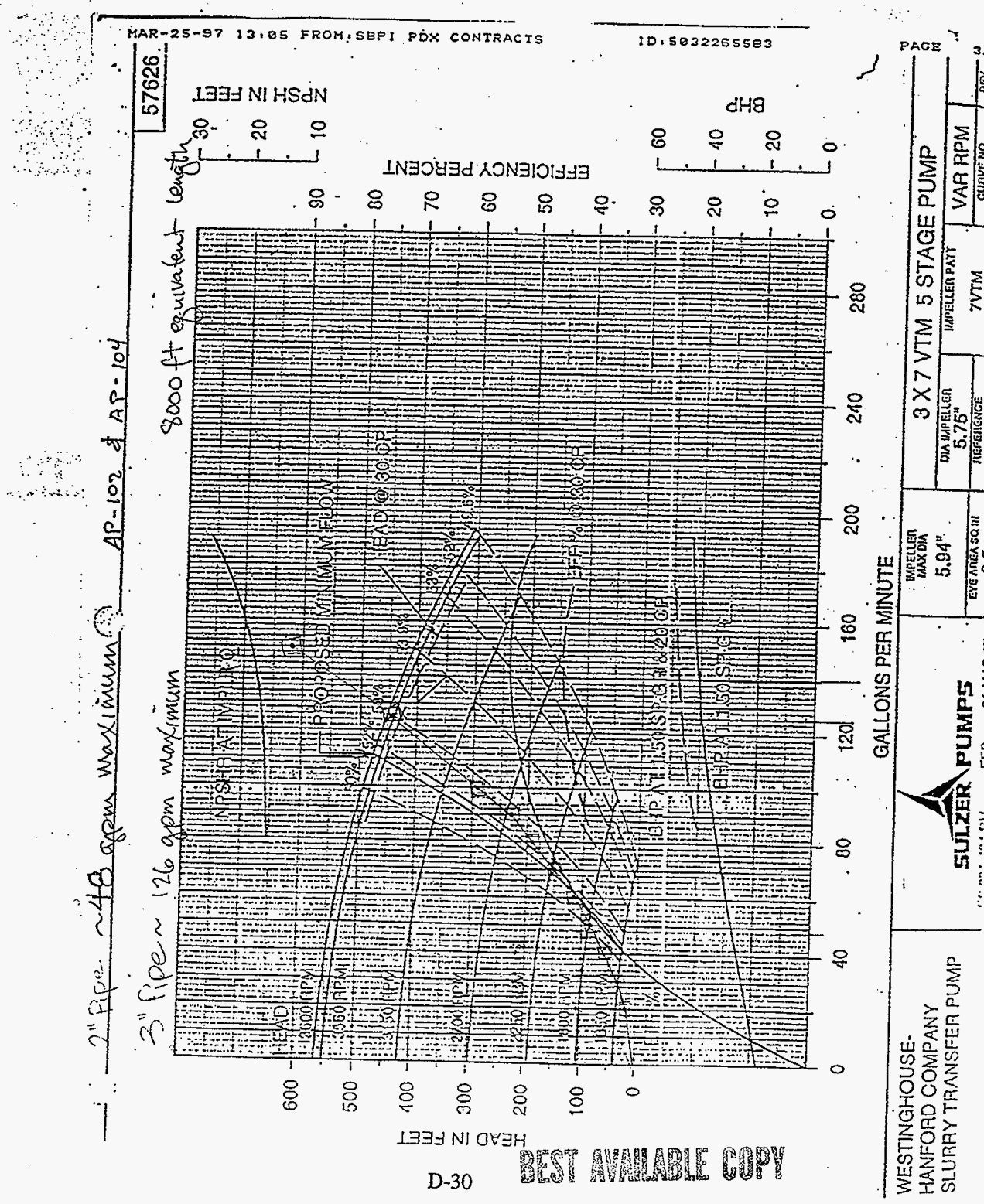


HNF-2238

Revision 0

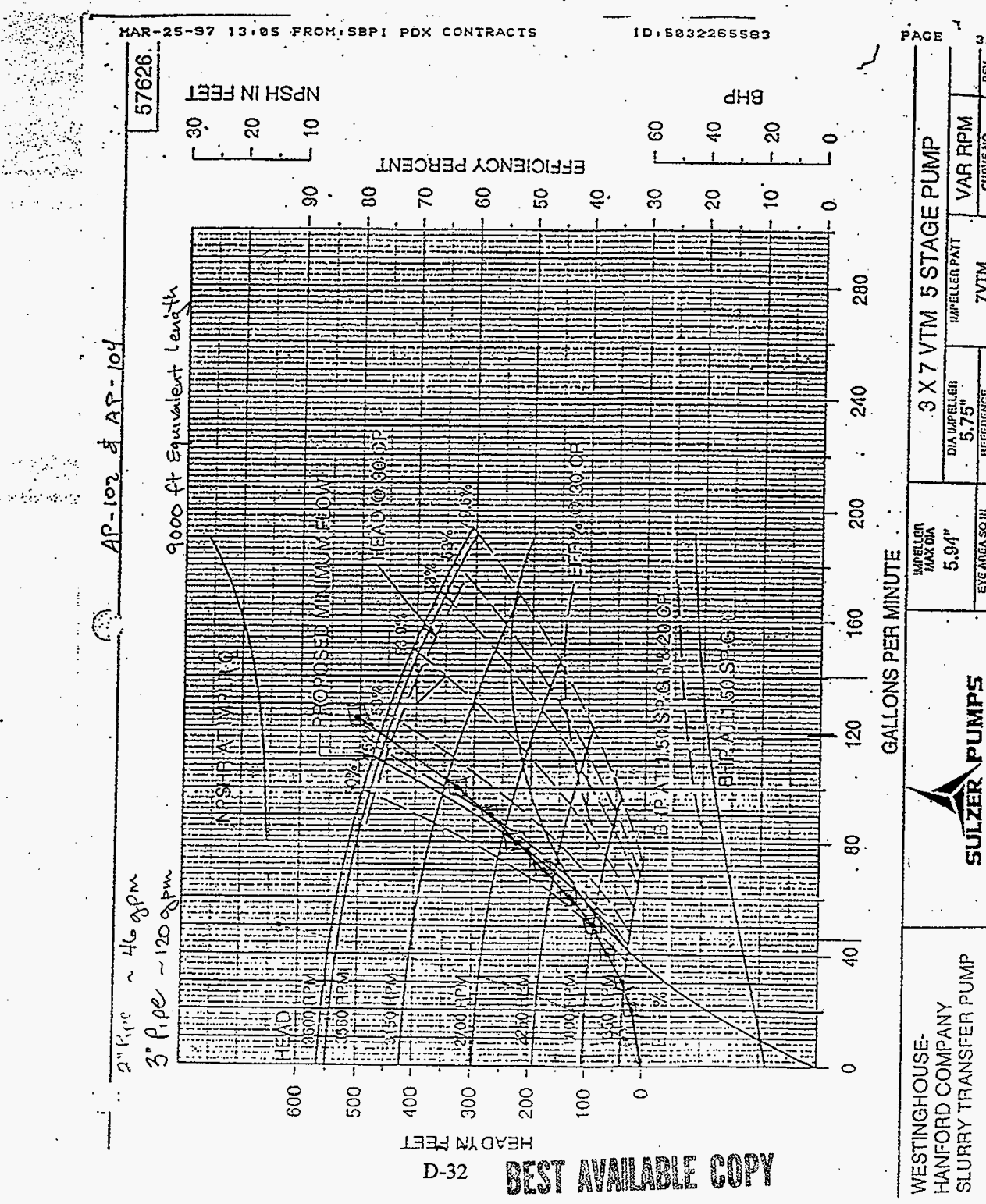




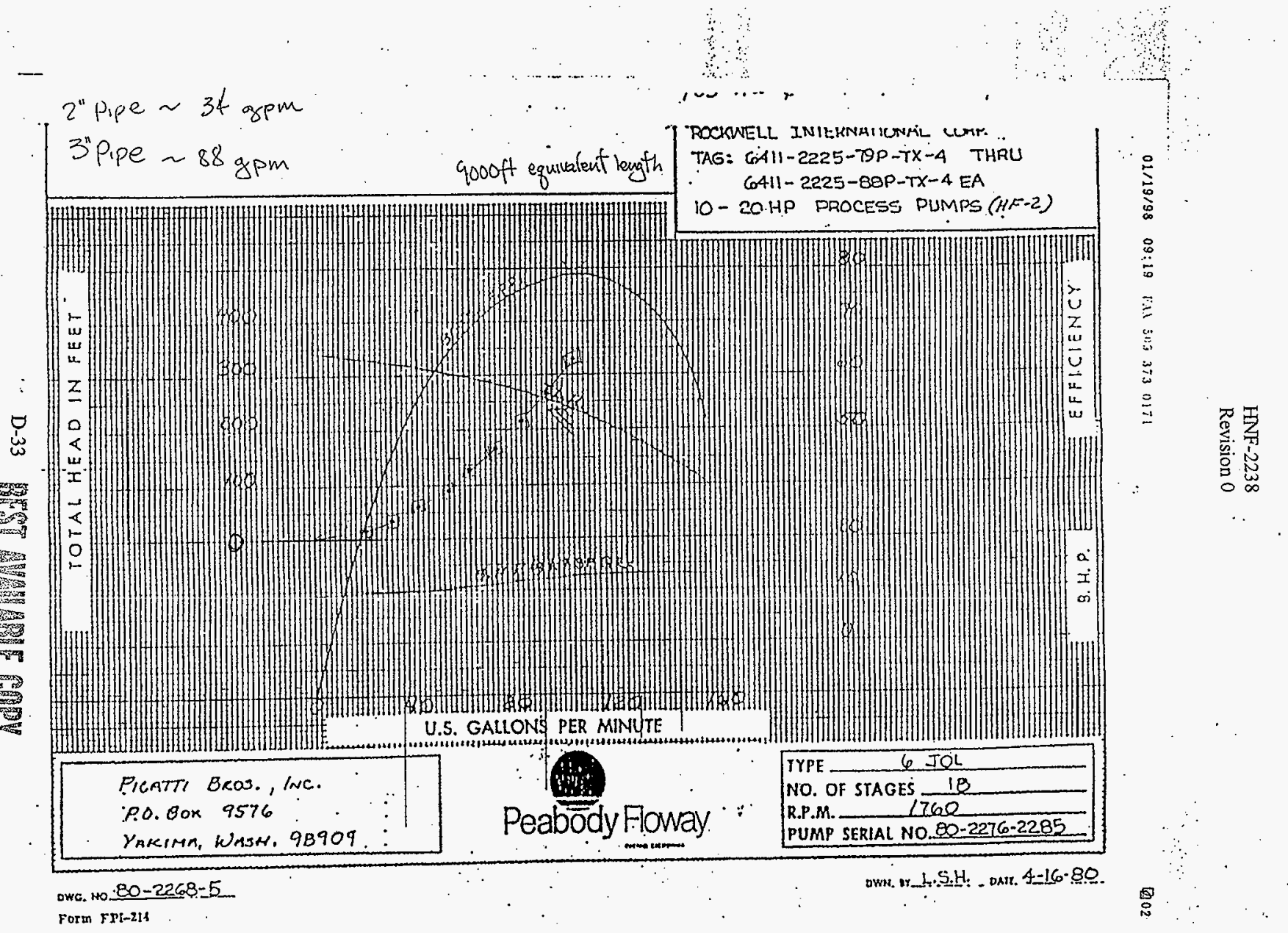


HNF-2238

Revision 0

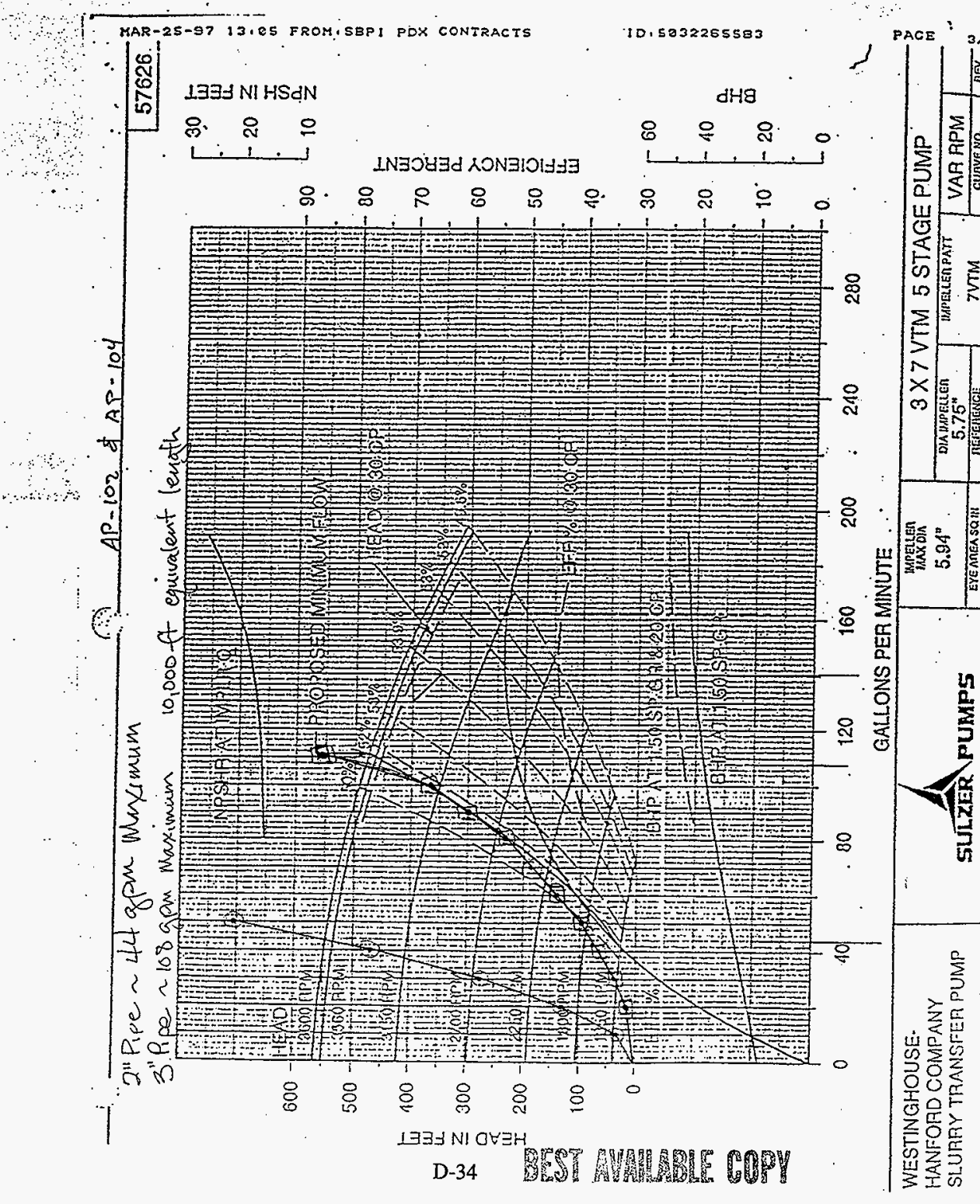




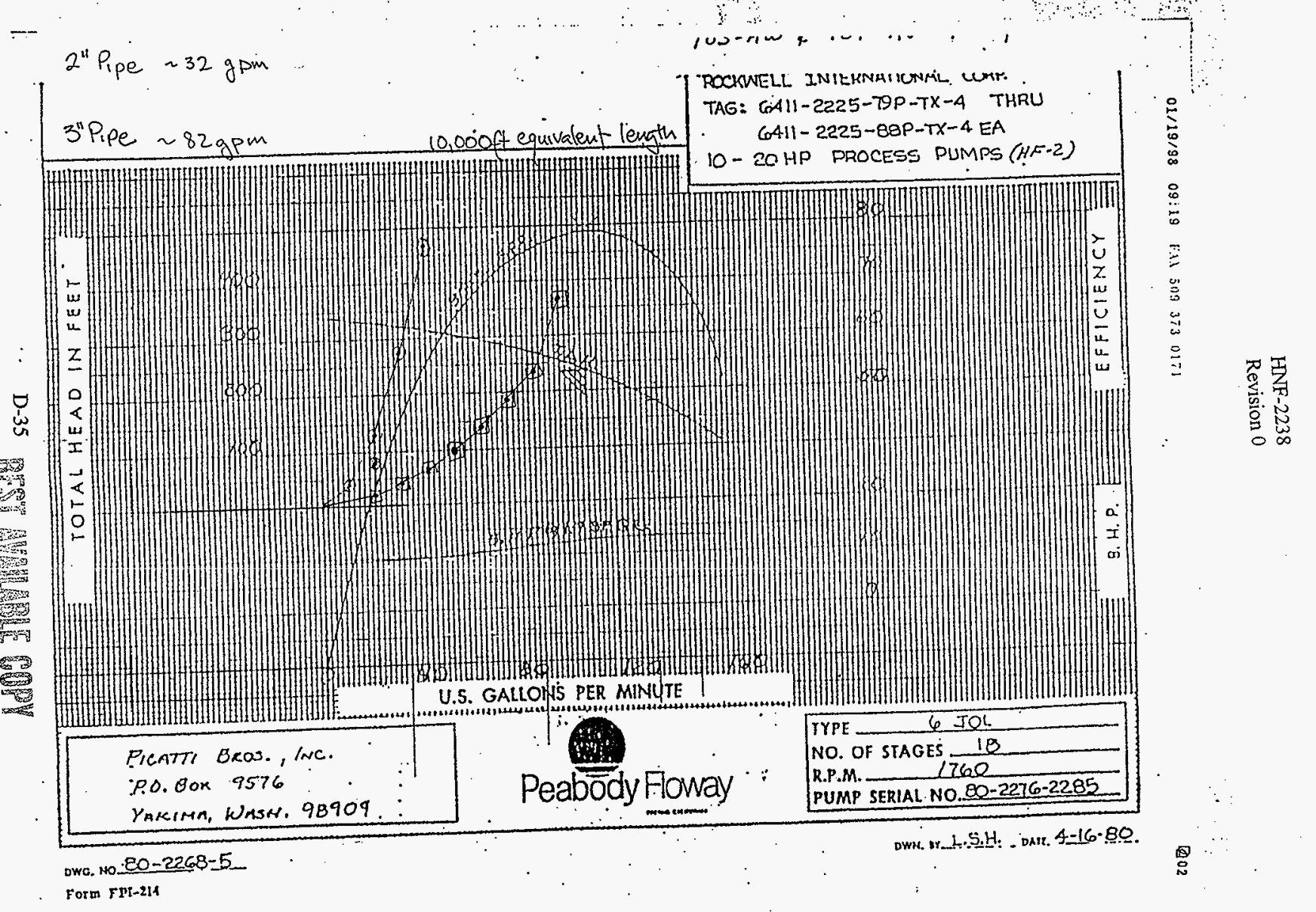


HNF-2238

Revision 0

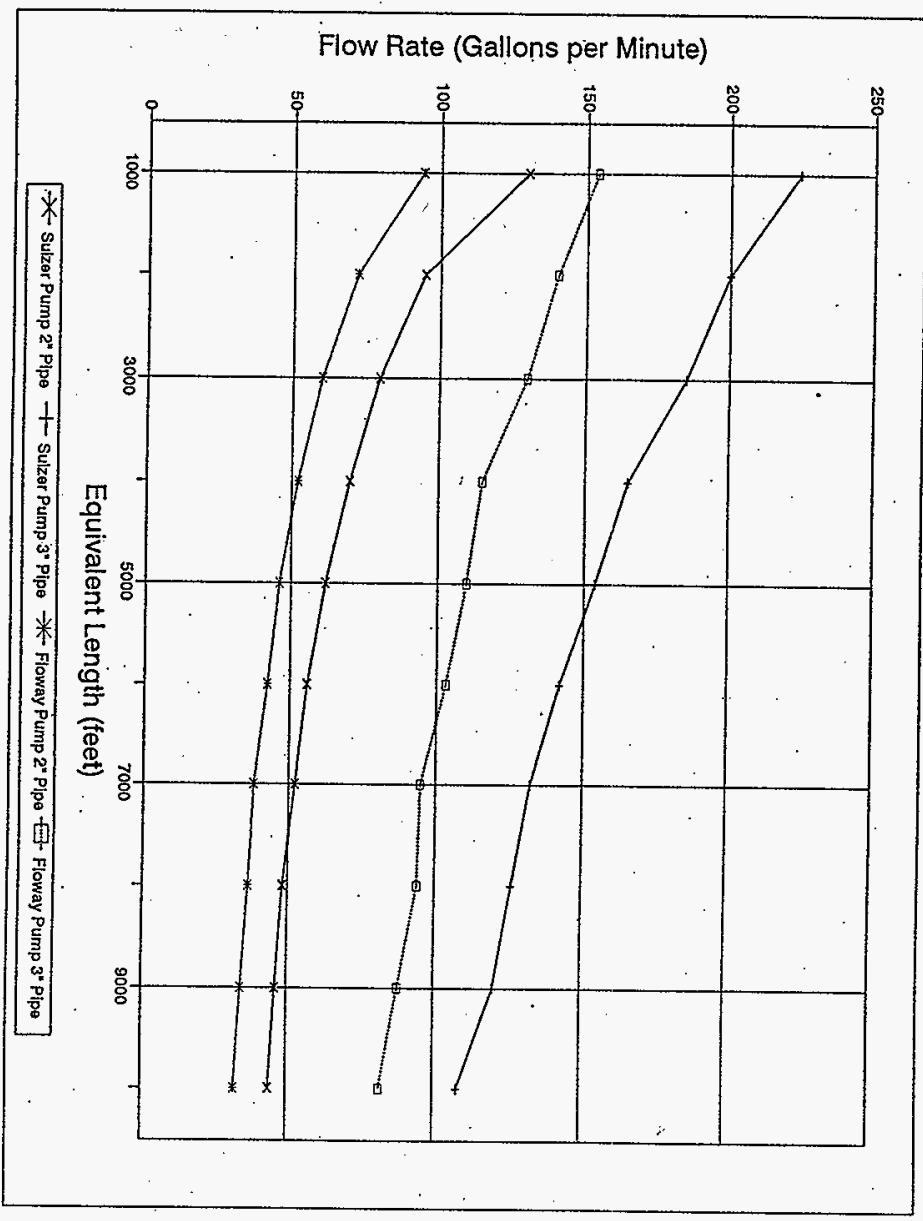




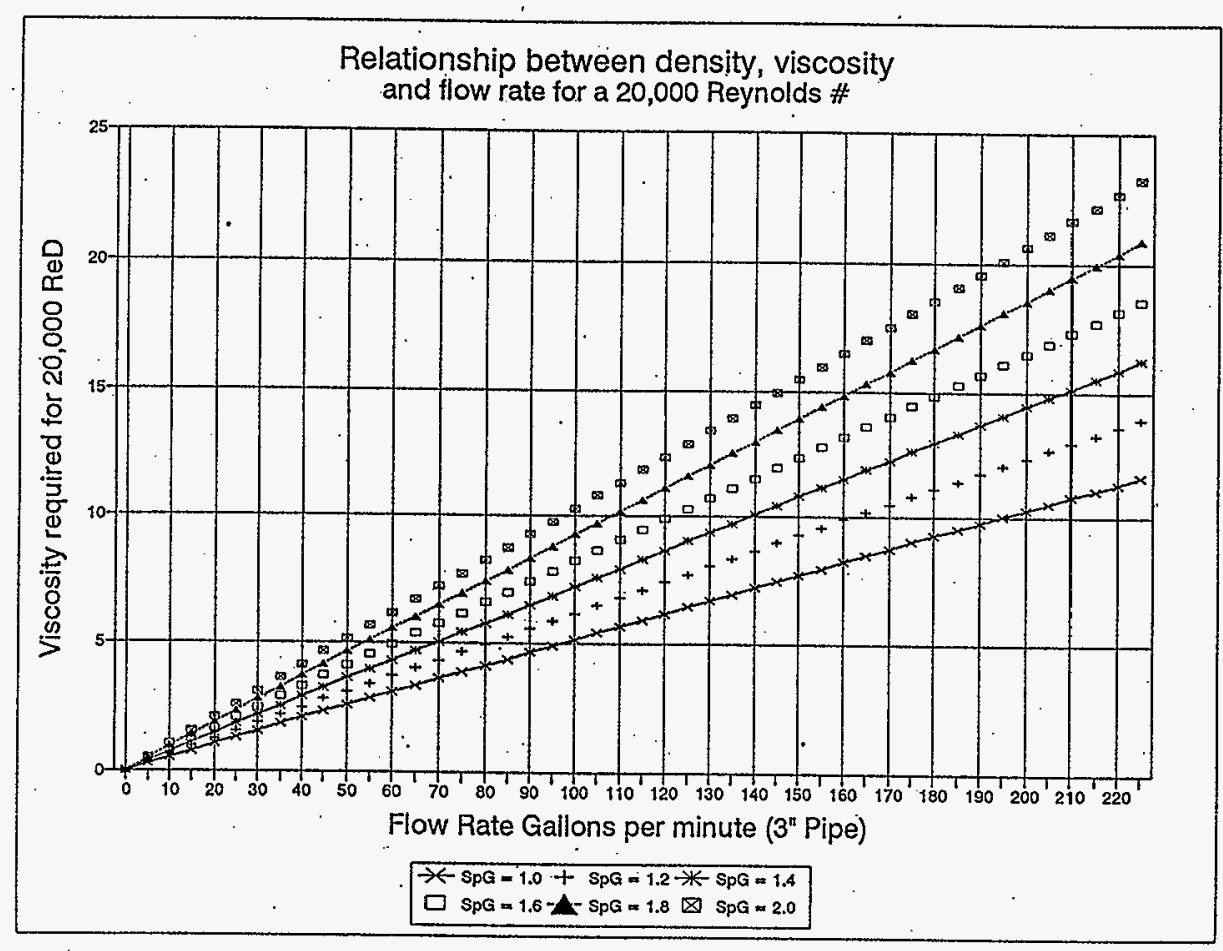

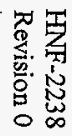


HNF-2238

Revision 0

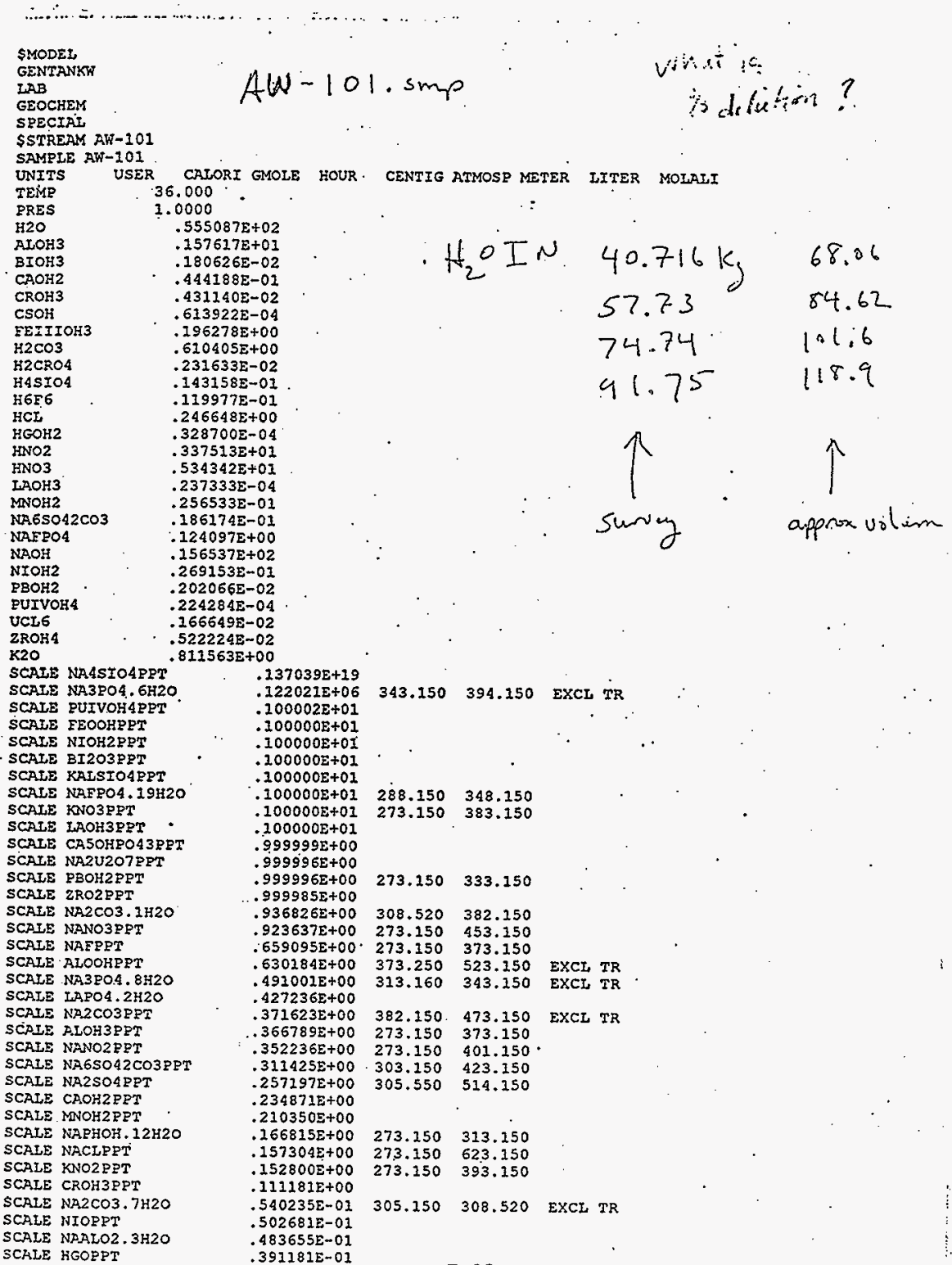


HNF-2238

Revision 0

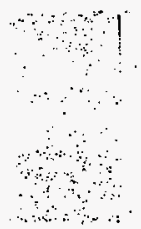

SCALE NAALSIOARPT

SCAIE KCLPPT

SCALE NA2CO3.10H2O

$.356196 \mathrm{E}-01$

.339694E-01

$.157802 \mathrm{E}-01$

$273.250 \quad 473.150$

SCALE NA2UOAPET

$.1265405-01$

$273.150 \quad 305.150 \quad$ EXCE TR

END

SSAMPLE Aंत- 102

DESC 2-Sep-97 single RecElec NoRecpH

UNITS USER CALORI GMOLE HOUR CENTIG ATMOSR METER IITER

TEMP 36.000

PRES 1.0000

AMH 1.0

DENS 1490.0

1433.8

PH $0.0 \quad 15.273$

RECELEC Make Up Ion Method using $-.5759 \mathrm{mg} / \mathrm{l}$ of OHION

EOUIJ IAAB BAIANCE OHION . OHION CONCENTRATION $\mathrm{mg} / 1$.

$\mathrm{AL}+3$

$\mathrm{BI}+3$

$\mathrm{CA}+2$

CRIII +3

CS+1

EEIII+3

HG +2

$\mathrm{K}+1$

$\mathrm{LA}+3$

$\mathrm{MN}+2$

$\mathrm{NA}+\mathrm{I}$

$\mathrm{NI}+2$

$\mathrm{PB}+2$

PUIV+4

Uo $2+2$

$\mathrm{ZR}+4$

$\mathrm{OH}-1$

CL-1

$\cos -2$

CRO4-2

E-1

H3SIO4-1

NO2-1

Nó3-1

PO4-3

504-2

SOL SCAIING

NA.4SIO 4 PPT

NA.3FO $4.6 \mathrm{H} 20$

PUIVOH 4 PPT

FEOOHPPT

NIOH2PPT

BI203PPT

KAISIOAPPT

NAEPO4. I9H2O

KNO3PET

IAOH 3 PPT

CA5OHPO43PRT.

NA2U207 $2 P T$

PBOH2PPT

ZRO2PPT

$\mathrm{NA} 2 \mathrm{CO} 3.1 \mathrm{H} 2 \mathrm{O}$

NANOSPFT

NAFPQT

ALOOHPPT

N.43P04.8H2O

IAPOA $2 \mathrm{H} 2 \mathrm{O}$

NA2CO3PFI

F.LOH3PPT

NANO2PPT

NA6SO42CO3PPT

NA2SO4PRT

CAOH2PPT

MNOH2PPT

NA.PHOH $12 \mathrm{H} 2 \mathrm{O}$

$\begin{array}{ll}25800 . & 25800 \\ 229.00 & 229.00 \\ 1080.0 & 1080.0 \\ 135.00 & 136.00 \\ 4.9500 & 4.9500 \\ 6650.0 & 6650.0 \\ 4.0000 & 4.0000 \\ 38500 . & 38500 . \\ 2.0000 & 2.0000 \\ 855.00 & 855.00 \\ 2.32005+05 & 2.32008+05 \\ 958.00 & 958.00 \\ 254.00 & 254.00 \\ 3.3200 & 3.3200 \\ 273.00 & 273.00 \\ 289.00 & 289.00 \\ 1.2947 E+05 & 1.29472+05 \\ 5520.0 & 5520.0 \\ 22900 . & 22900: \\ 163.00 & 163.00 \\ 2260.0 & 2260.0 \\ 826.00 & 826.00 \\ 94200 . & 94200 \\ 2.01008+05 & 2.01008+05 \\ 14300 . & 14300 \\ 2170.0 & 2170.0 \\ & \end{array}$

$.1370395+19$

$.122021 \mathrm{E} \div 06$

$.100002 E+01$

$.100000 \mathrm{E}+01$

$100000 \mathrm{E}+01$

$.100000 E+01$

$.100000 E+01$

$.1000002+01$

$100000 \mathrm{E}+01$

$.100000 z+01$

$.999999 \mathrm{E}+00$

$.999996 \mathrm{E}+00$

$.999996 \mathrm{E}+00$

$.999 .985 E+00$

$.936826 \mathrm{E}+00$

$.923637 E+00$

$.659095 \mathrm{2}+00$

$.630184 \mathrm{E}+00$

$.491001 \mathrm{E}+00$

$427236 E+00$

$.371623 \mathrm{E}+00$

$.366789 E+00$

$.352236 \mathrm{E}+00$

$311425 \mathrm{E}+00$

$.2571972+00$

$.234871 \mathrm{E}+00$

$.210350 E+00$

$.266815 \mathrm{~F}+00$
$288.150 \quad 348.150$

$273.150^{\circ} \quad 383.150$

$273.150 \quad 333.150$

$308.520 \quad 382.150$

$273.150 \quad 453.150$

$273.150 \quad 373.150$

$372.250 \quad 523.150$

$313.160 \quad 343.150$

$382.150 \quad 473.150$

$273.150 \quad 373.150$

$273.150 \quad 401.1 .50$

$303.150 \quad 423.1 .50$

$305.550 \quad 514.1 .50$

$273.150 \quad 313.150$
EXCL TR

EXCL TR

EXCL TR
D-39 
HNF-2238

Revision 0

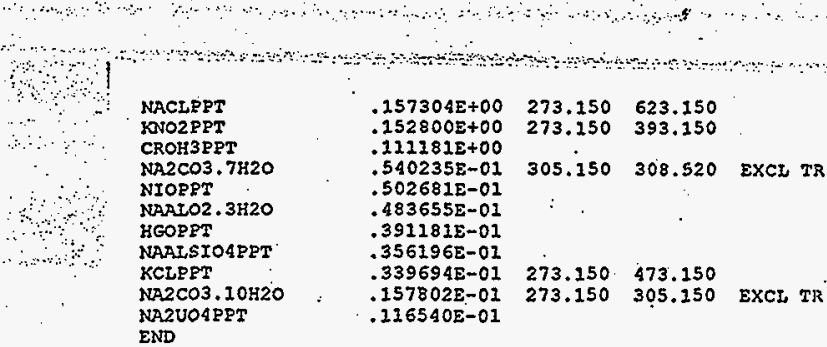


HNF-2238

Revision 0

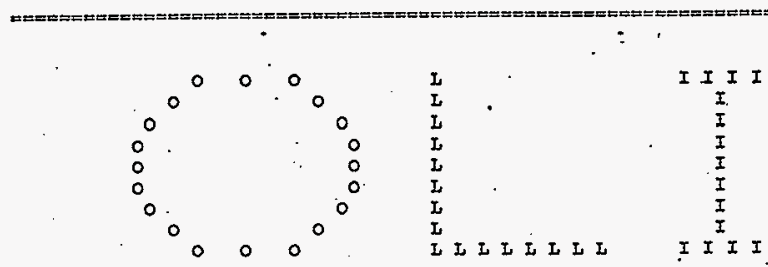

ENVIRONMENTALSIMUIATION PROGRAM

$$
v-5.4 \quad \text { March 1, } 1997
$$

SURVEY: AW-101

CHEMISTRY MODEL: gentank

THIS FILE NAME: AW-201. Sum

DATE: $\quad 3-0 c t-97$

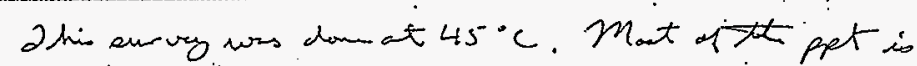

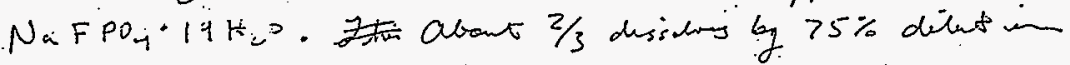
Alow, $m_{n}(\mathrm{OH})_{2}$ byins to formit at $25 \%$ delut $=\mathrm{KNO}_{3}$ is almet at sat as as $\mathrm{Na}_{2} \mathrm{CO}_{3} \cdot \mathrm{H}_{2}$.

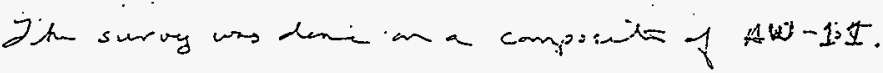

$$
\begin{aligned}
& \text { dnitios solic } 3.47 \mathrm{~kg} / 10 \mathrm{~s} \text { is canpocit } \\
& 3.27 \text { j e } 25 \% \text { dilut } \\
& \text { 2. } 82,3<5 \text { \& dinti- } \\
& 2.05 \text { if } 475 \% \text { dilution. }
\end{aligned}
$$


HNF-2238

Revision 0

TABLE OE CONTENTS

STREAM SECTION

SURVEY VARIABIES SECTION

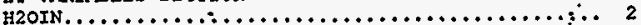

DENSITY. $\ldots \ldots \ldots \ldots \ldots \ldots \ldots \ldots \ldots \ldots \ldots \ldots \ldots \ldots \ldots \ldots \ldots, 2$

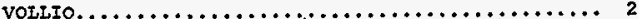

NAEPO $4.19 \% 20, \ldots \ldots \ldots \ldots \ldots \ldots \ldots \ldots \ldots \ldots \ldots \ldots \ldots \ldots \ldots, 2$

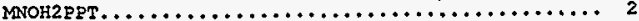

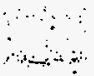


HNF-2238

Revision 0

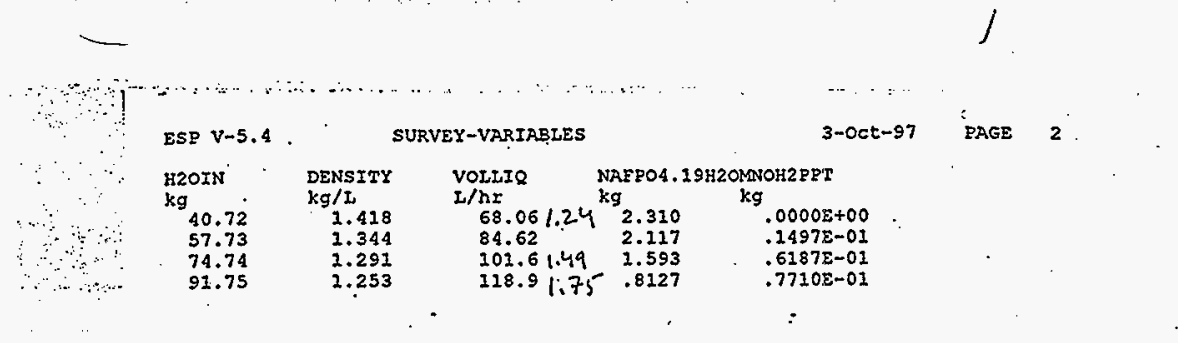

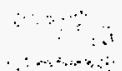


HNF-2238

Revision 0

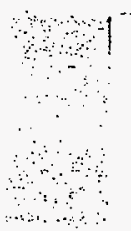

EsP $V-5.4$

STREAM:AH-101

3-0ct-97 PAGE

STREAM: AW-102

POINT : 1

Phases--n-mas

Temperature, c

Eressure, atm

pH

Total mol/hr

H2O
ALOH3
BIOH3
CASO4
CO2
CSCL
CSNO3

FEIIIOH3

H3PO4

HGCE2

HGOH2

HNO2

KCL

LAF3

IAOH 3

MNNO32

MNOH2

NAT

$\mathrm{NAF} C \mathrm{CO}$

NANOS

NIOH2

PBNO22

PEO

PUIVOH4

$\mathrm{SzO} 2$

ZROH4

OHION

AIOH2ION

ALOH4ION

ALOHION:

BIOH4ION

CAEION

CAHCOSION

CAION

CAOHION

CAPO4ION

CIION

COSION

CR2O7ION

CROAION

CROHAION

CSION

CSSOAION

FEIIIOH2ION

FEIIIOH4ION

EEIIIOHION

FION

Aqueous

45.000

1.0000

14.965

3984.3

$\mathrm{kg} / \mathrm{hr}-$

$1.0116 \mathrm{E}-09$

5.3175E-07

$6.24442-12$

5. $0653 E-17$

$9.5039 \mathrm{E}-06$

1. $0914 \mathrm{E}-04$

$3.3155 \mathrm{E}-12$

$5.4868 \mathrm{E}-28$

7.57375-17

5. $9210 \mathrm{E}-06$

4. $6374 \mathrm{E}-13$

I. $5841 \mathrm{E}-03$

2.3651E-28

5:6414E-13

1. $8800 \mathrm{E}-15$

1. $5323 \mathrm{E}-08$

$2.9393 \mathrm{E}-02$.

3.96735-07

.13858

2.7905E-09

2. $3004 \mathrm{E}-16$

5.62978-09

$1.0653 \mathrm{E}-09$

1. $0561 \mathrm{E}-12$

4. $5314 \mathrm{E}-13$

3.7738 .

$6.6265 \mathrm{E}-19$

6.0421

$9.6400 E-30$

5. $8109 \mathrm{E}-03$

$1.4056 \mathrm{E}-11$

$6.7663 \mathrm{E}-17$

1. $6289 \mathrm{E}-08$

5. $9904 \mathrm{E}-07$

1. 8154E-05

.36972

1.0625

$1.8635 \mathrm{E}-21$

1. $0939 \mathrm{E}-02$

2. $1070 \mathrm{E}-02$

2. $5002 \mathrm{E}-04$

$4.4884 \mathrm{E}-07$

$2.20868-21$

$5.5430 \mathrm{O}-05$

2. $1492 \mathrm{E}-32$

$1.5004 \mathrm{E}-02$

1. $6723 \mathrm{E}-13$

3. $8707 \mathrm{E}-06$

H3SIOAION

HCOSION

HCROA ION

HGCL3ION

$9.4094 \mathrm{E}-07$

1. 2959E-12

7. $518.7 \mathrm{E}-15$

2. $7273 \mathrm{E}-13$

$9.6762 \mathrm{E}-22$

HGCLION

HGFION

HGION

HGOH3ION

$5.0694 \mathrm{E}-29$

3. $4690 E-28$

3. 3039E-04

6. $6107 \mathrm{E}-17$

HGOHION

2. $2344 E-17$

HION

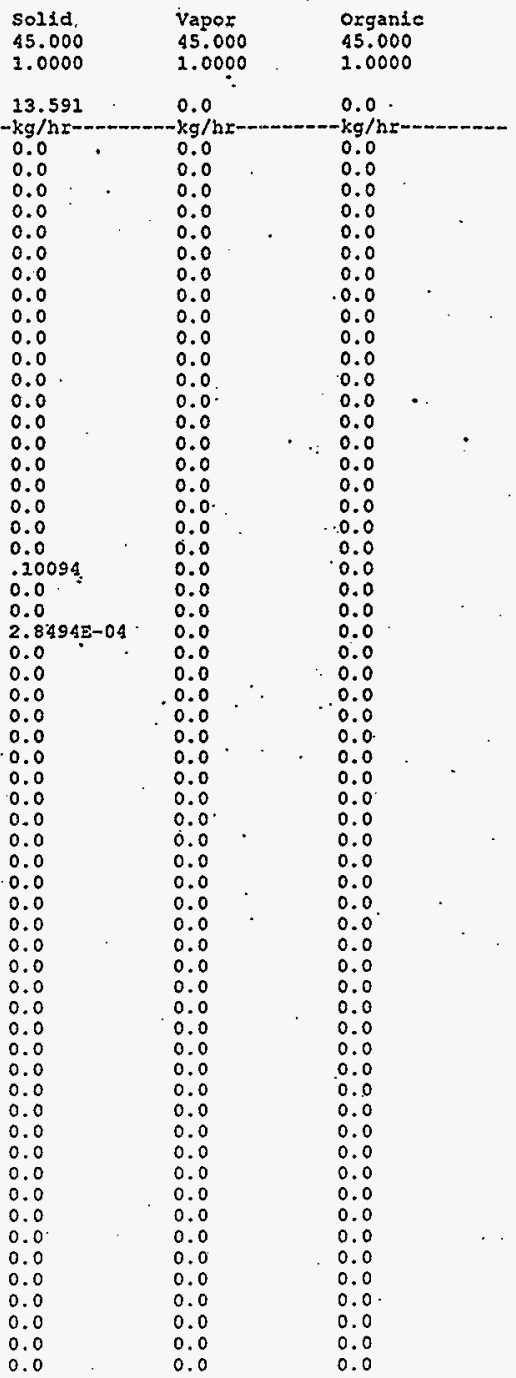

D-44 
HNF-2238

Revision 0

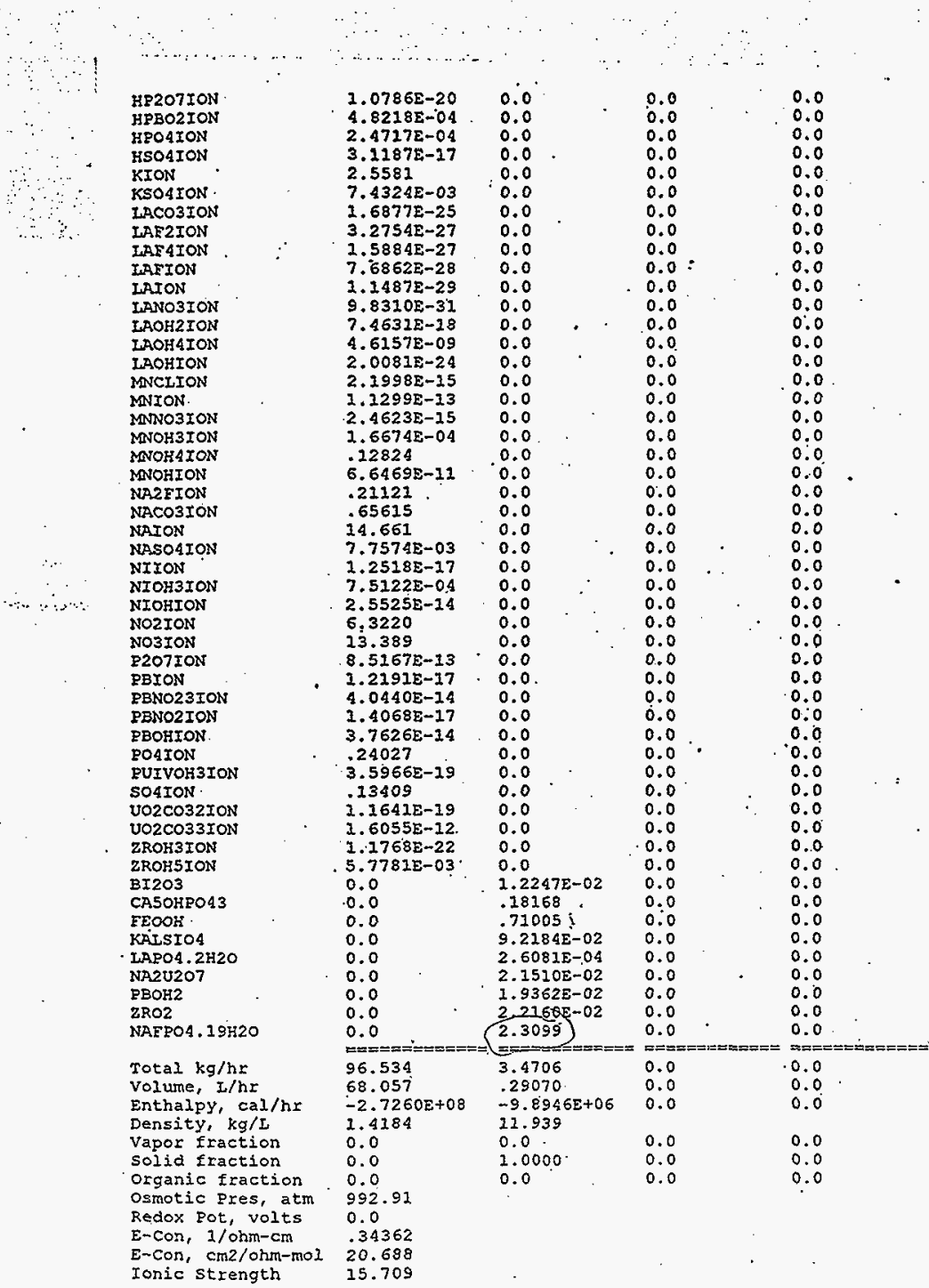


HNF-2238

Revision 0

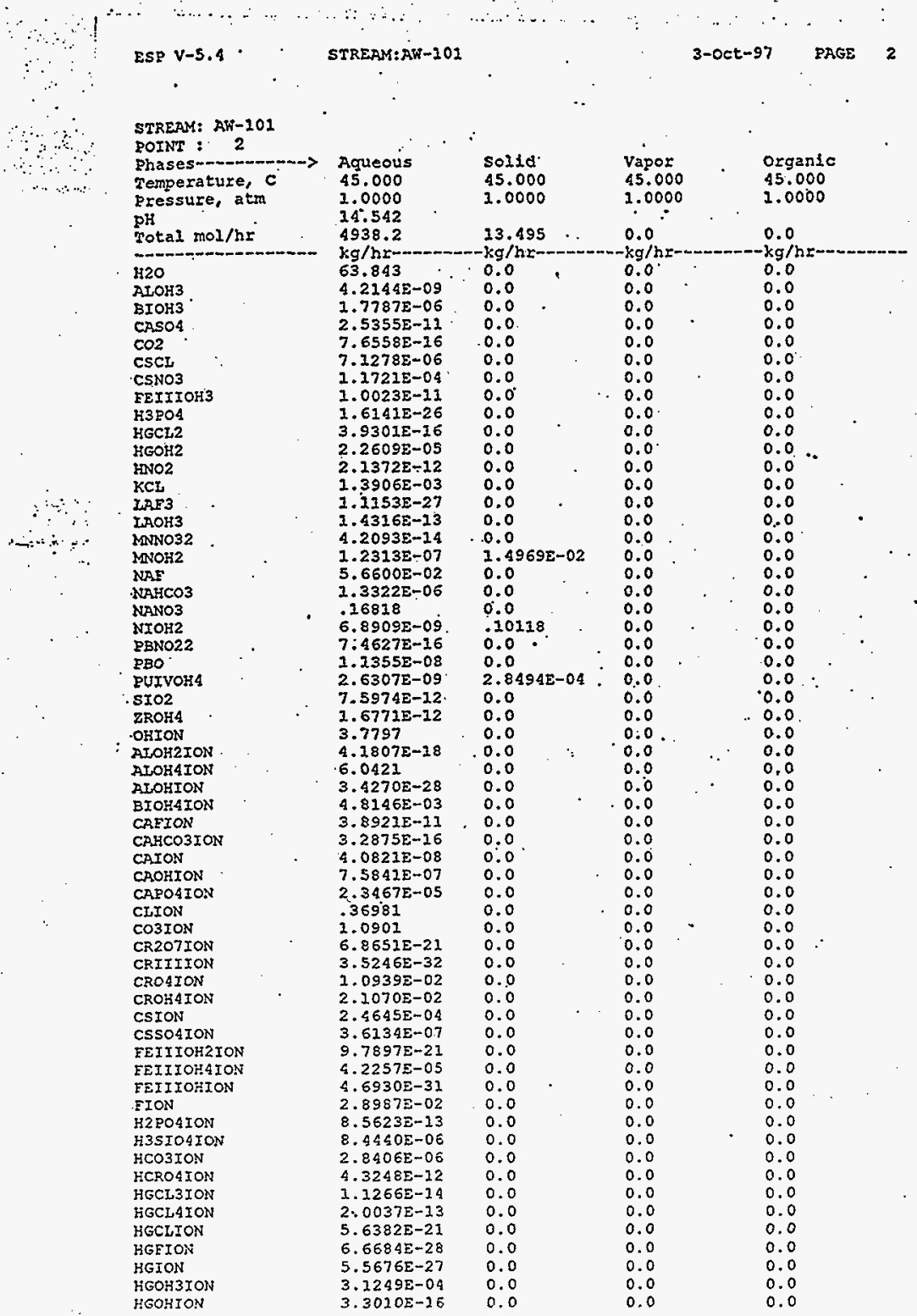


HNF-2238

Revision 0

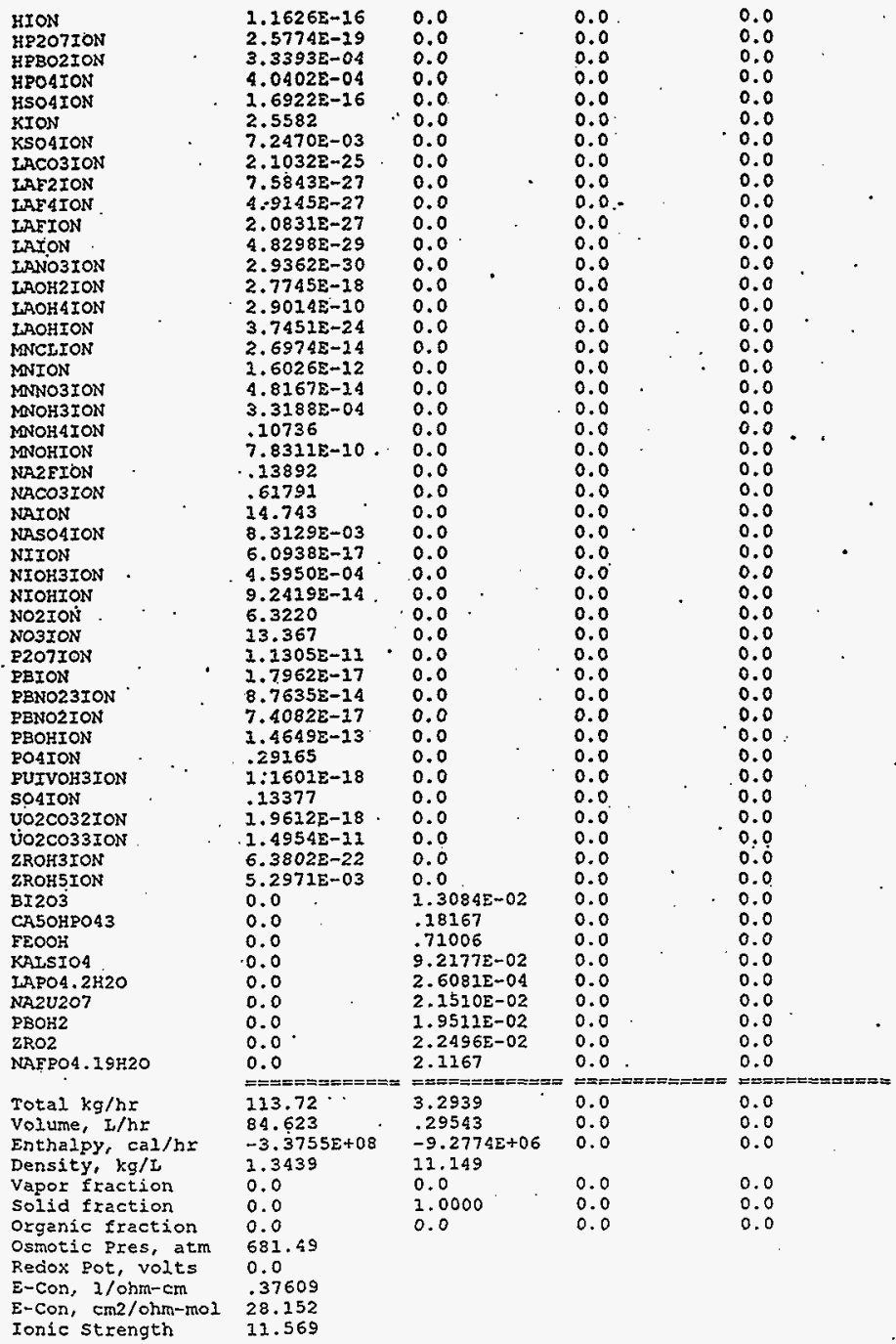


HNF-2238

Revision 0

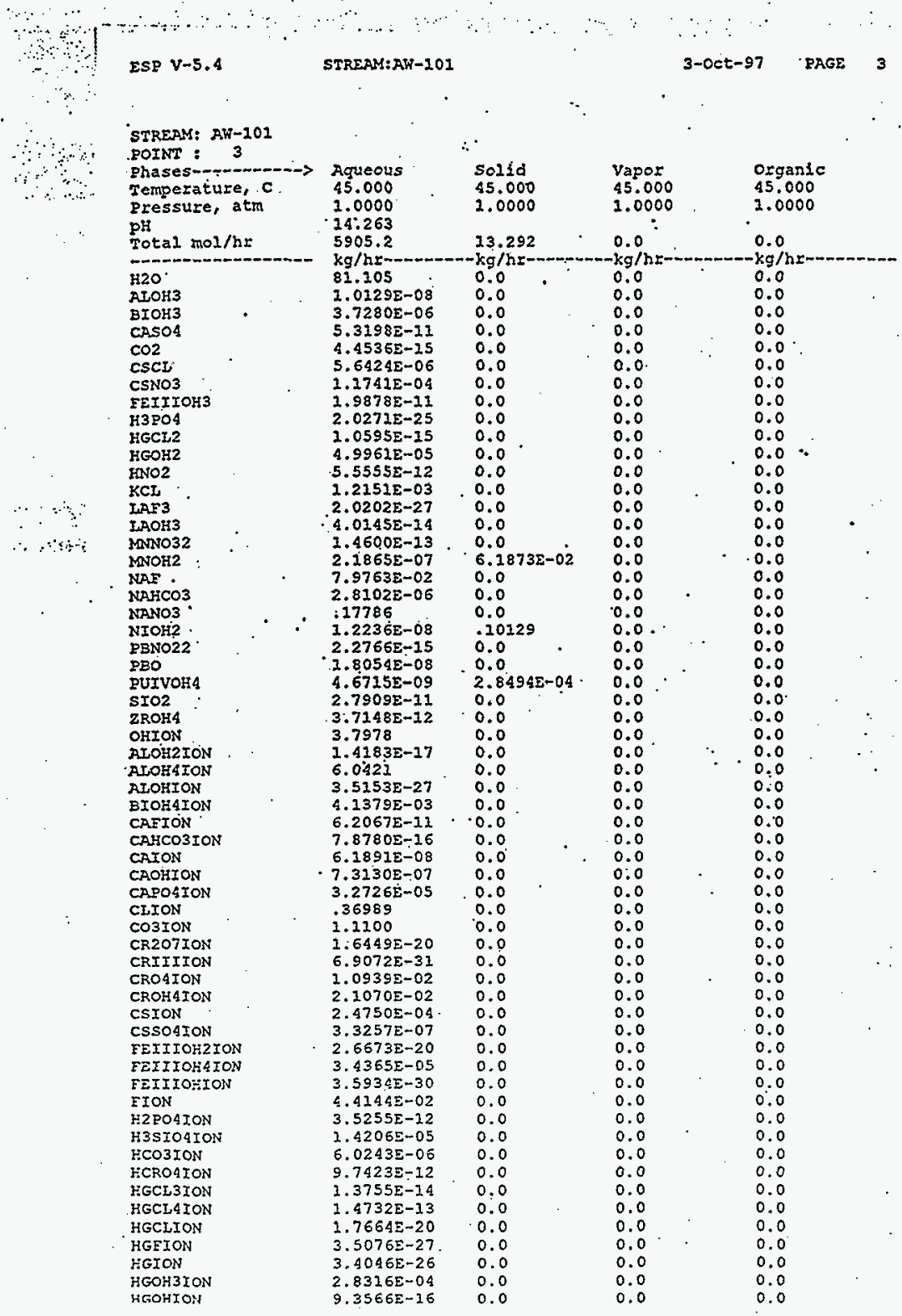


HNF-2238

Revision 0

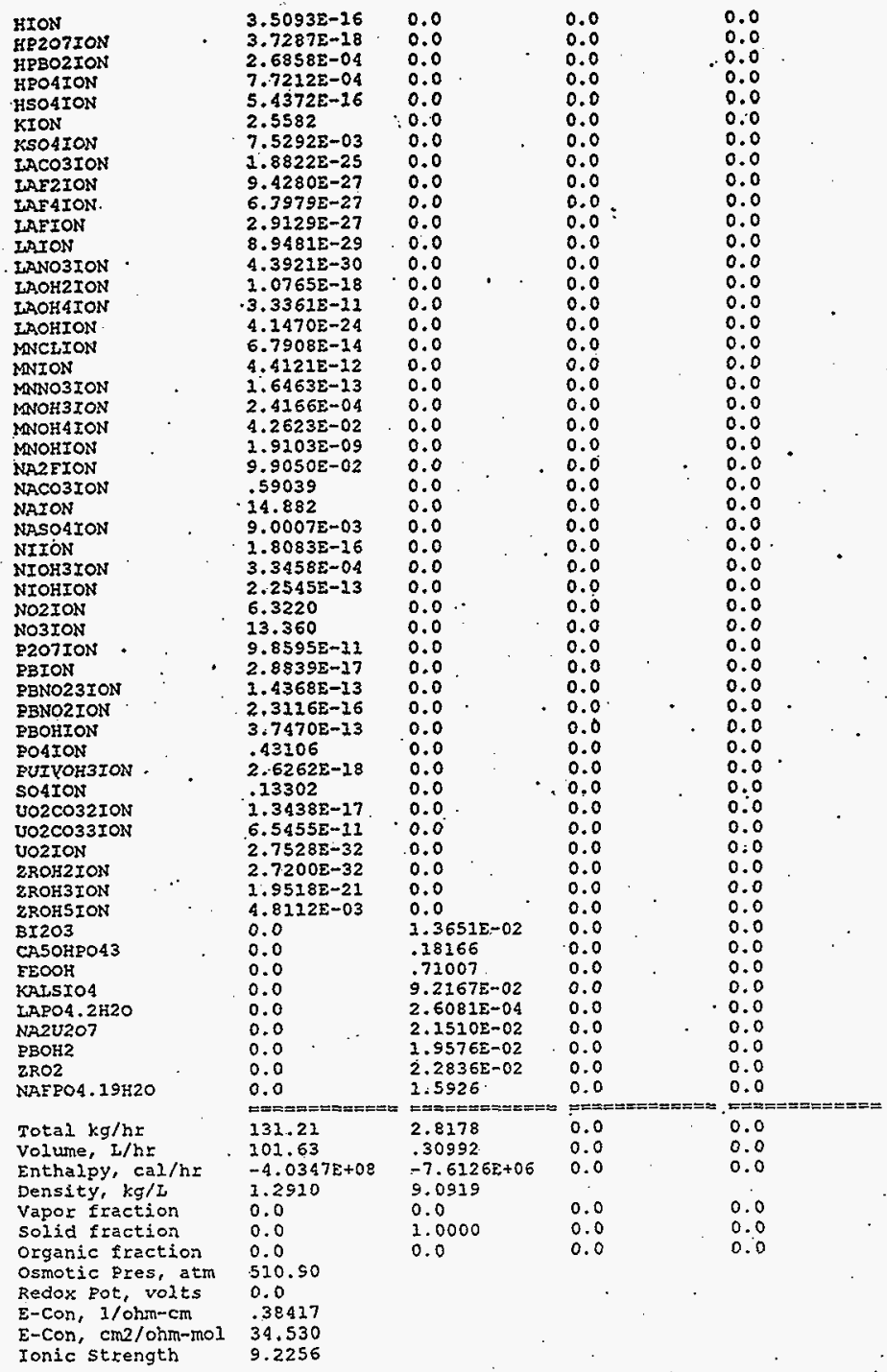


HNF-2238

Revision 0

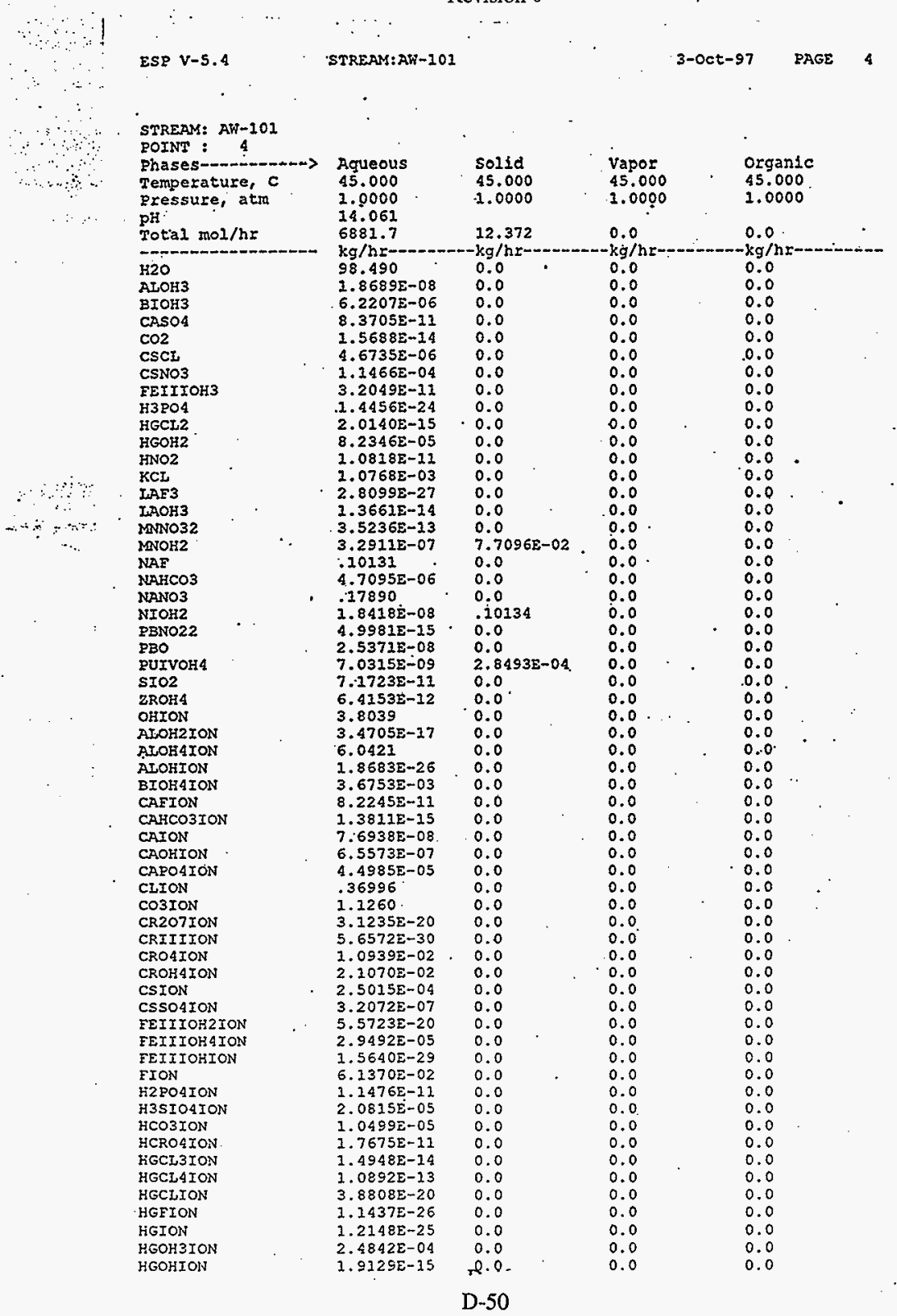


HNF-2238

Revision 0

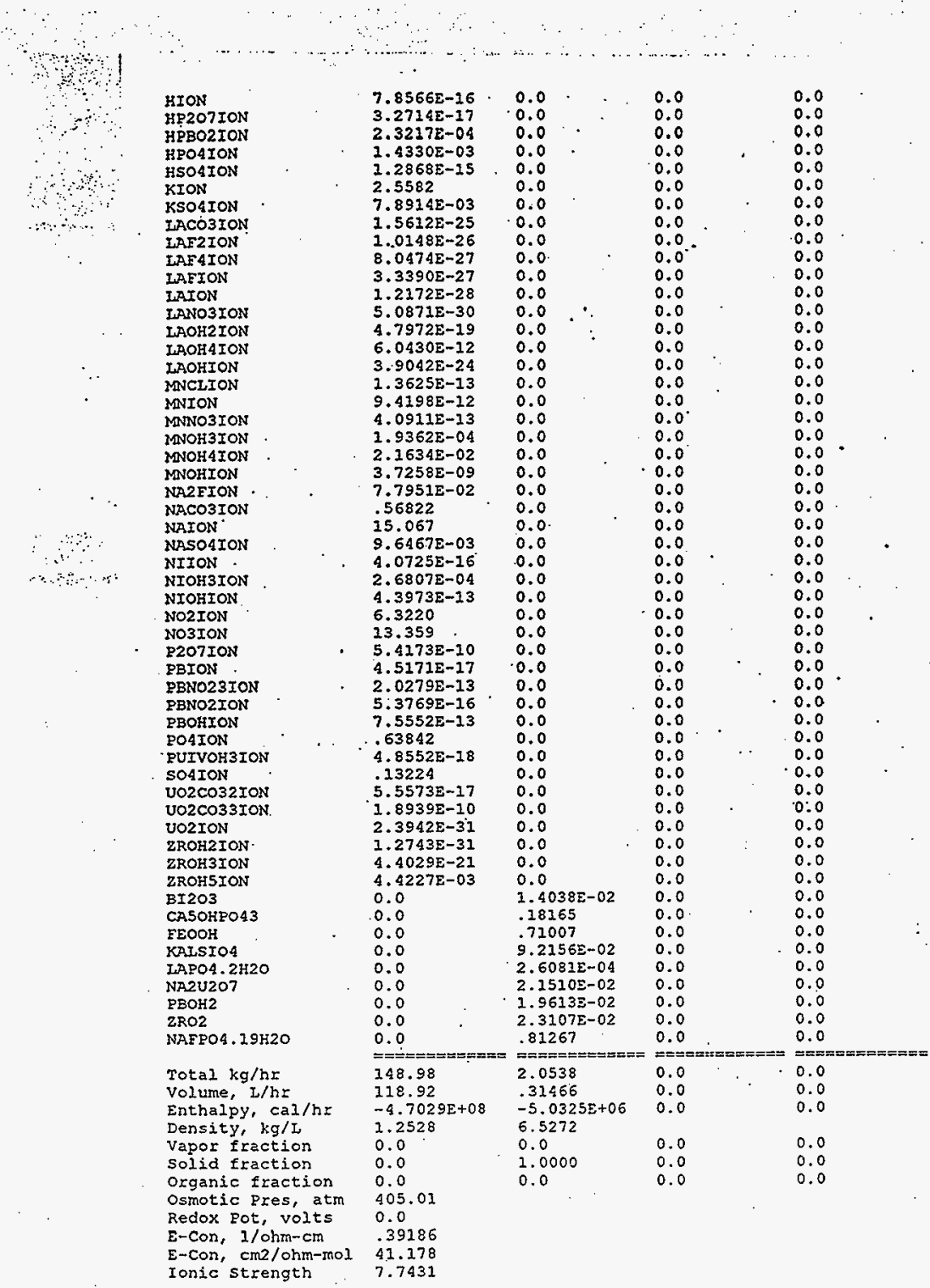


HNF-2238

Revision 0

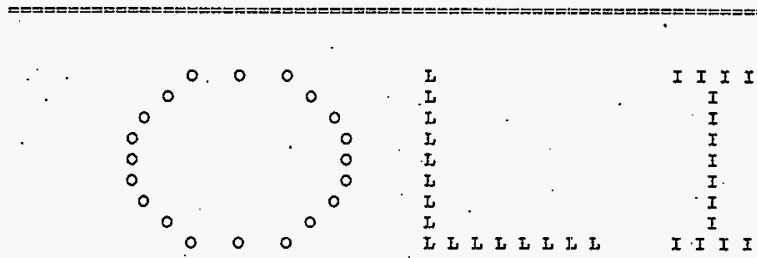

ENVIRONMENTALSIMUIATIION PROGRA $\mathrm{V}-5.4 \quad$ March 1, 1997

SURVEY: $A P-101$

CHEMISTRY MODEL: gentank

THIS FILE NAME: AP-101.IIS

DATE: 6-Feb-98 
HNF-2238

Revision 0

ESP V -5.4

STREAM: AP-101

6-Feb-98 " PAGE 1

STREAM: ap-101

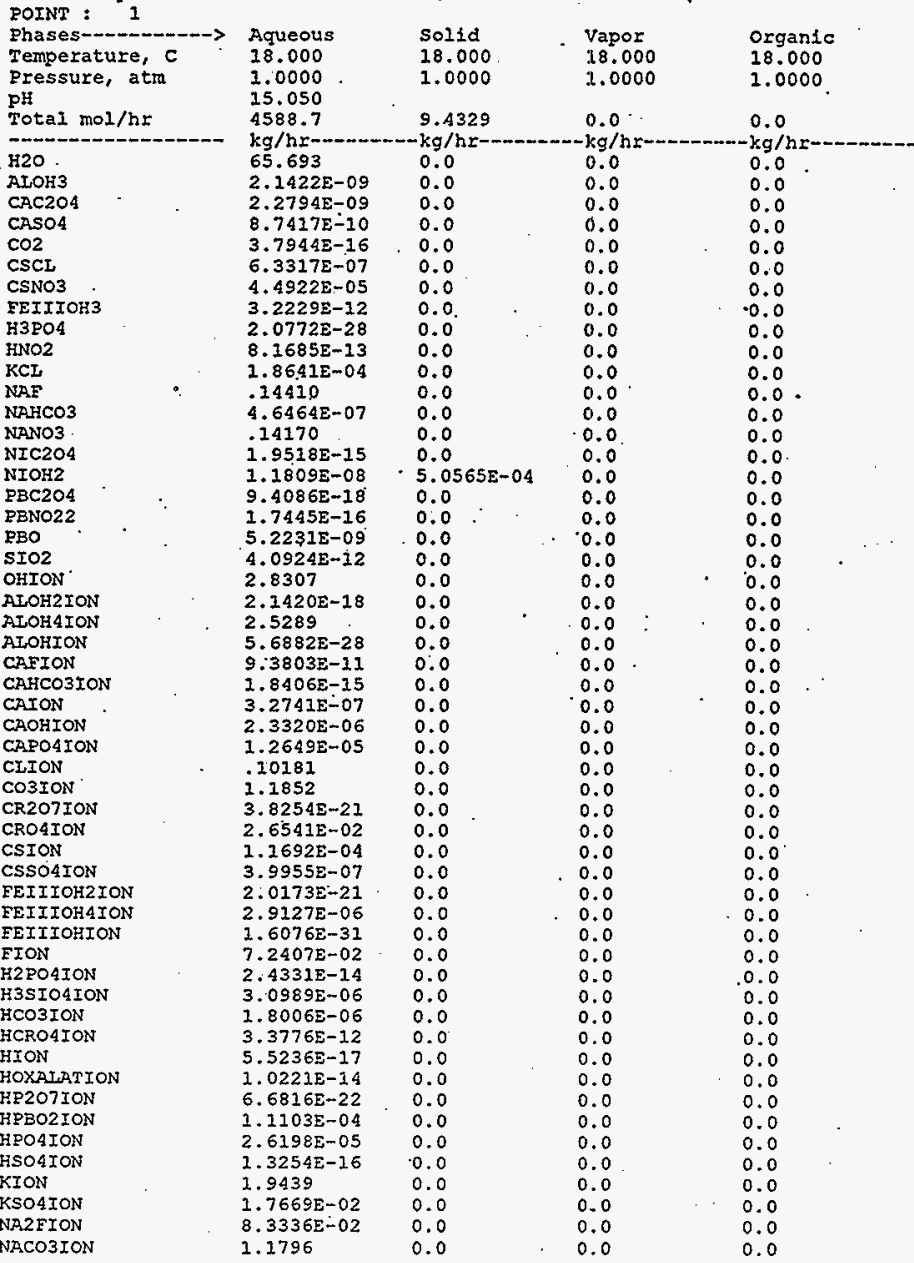


HNF-2238

Revision 0

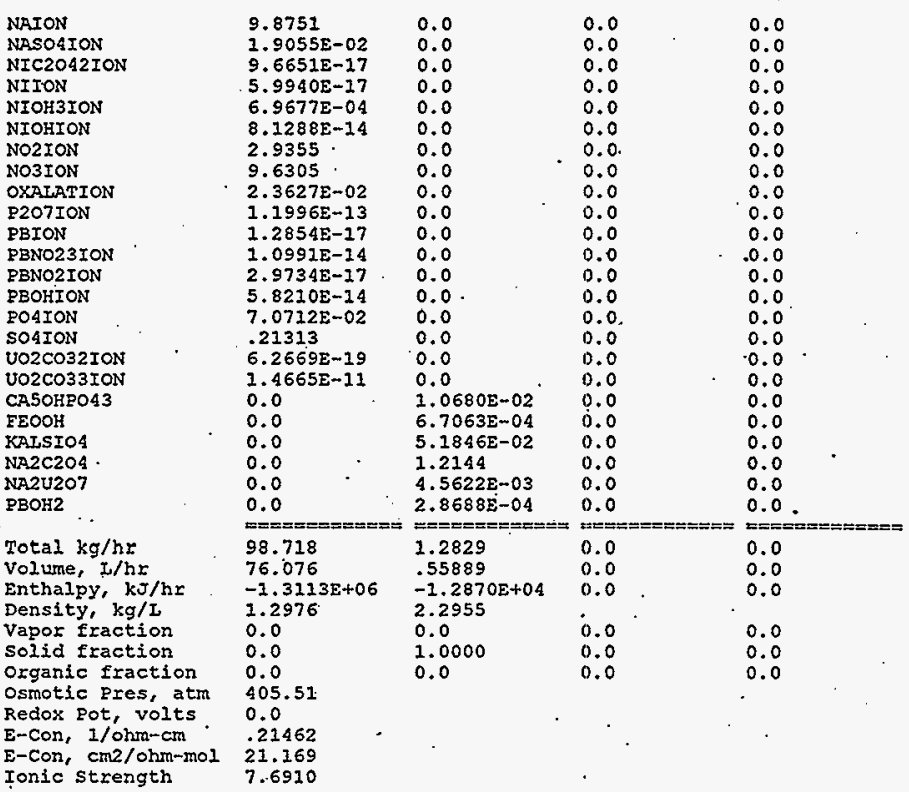


HNF-2238

Revision 0

ESP $V-5.4$

STREAX: ap-101

6-Feb-93 PAGE 2

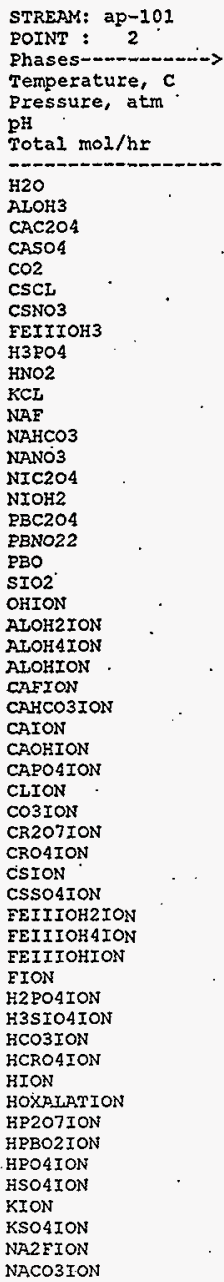

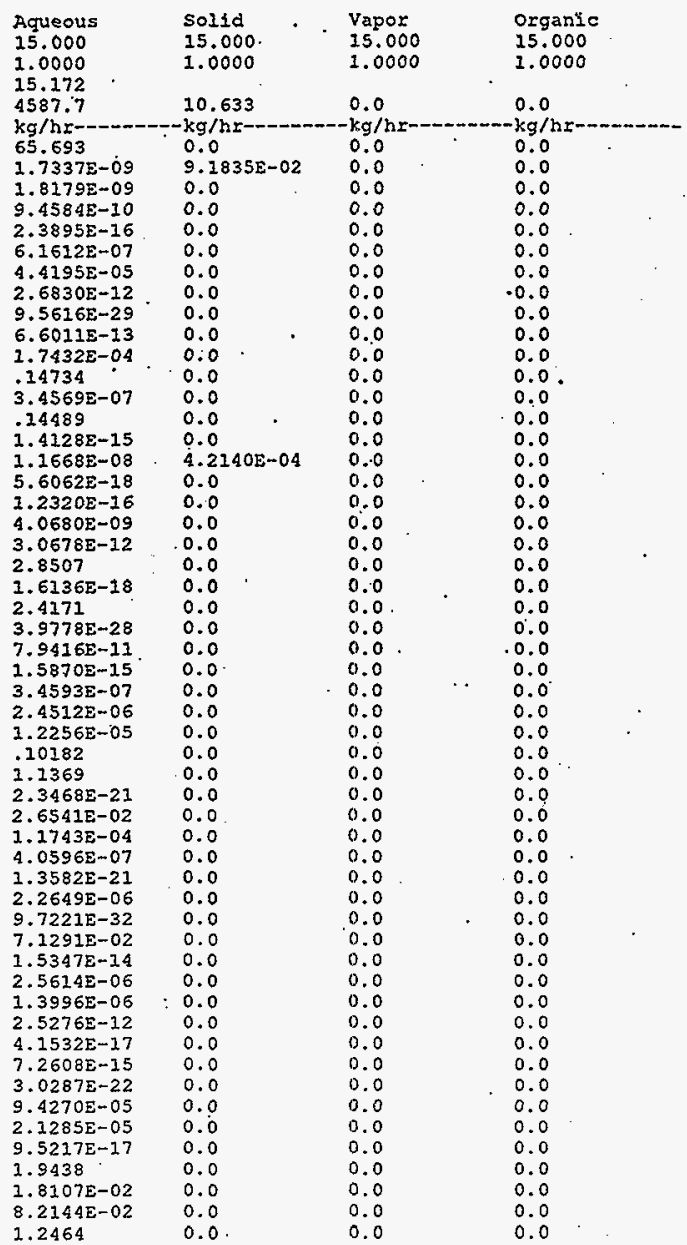


HNF-2238

Revision 0

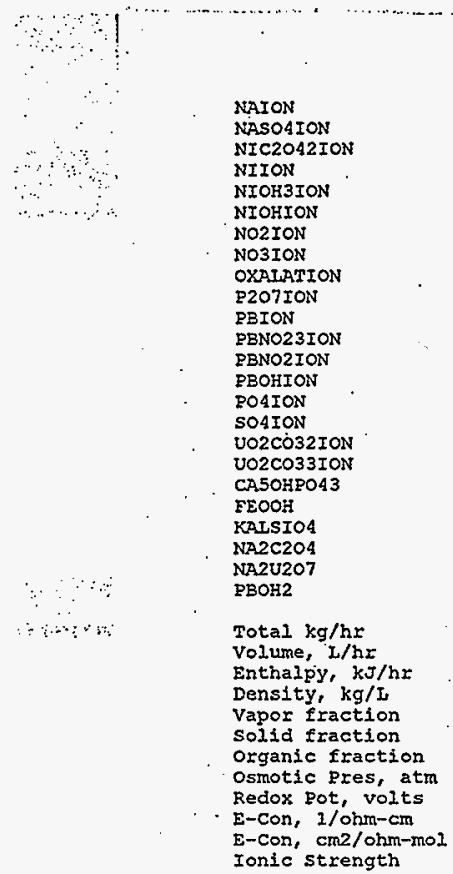

9.8536

$3.9300 \mathrm{E}-02$

7.0442E- 17

$4.9001 E-17$

$7.96488-04$

$6.9175 \mathrm{E}-14$

2.9355

9.6281

2.15185-02

$7.1937 \Sigma-14$

$9.4388 \mathrm{E}-18$

8.5690E-15

2. $08095-17$

$3.99805-14$

$7.0718 \mathrm{E}-02$

.21262

3.3743E-19

8. $9839 \mathrm{E}-12$

0.0

0.0

0.0

0.0

0.0

$0.0^{\circ}$

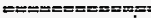

98.623

75.728

$-1.3106 \mathrm{E} \div 06$

1.3023

0.0

0.0

0.0

405.33

0.0

.19809

19.480

7. 6638
$0.0 \quad \cdot 0.0$

$0.0 \quad 0.0$

$0.0 \quad 0.0$

$0.0 \quad 0.0$

$0.0 \quad 0.0$

$0.0 \quad 0.0$

0.0

0.0

0.0

0.0

0.0

0.0

0.0

0.0

0.0

0.0

0.0

0.0

1. $0680 \mathrm{E}-02$

$6.7109 \mathrm{E}-04$

5. $1847 \mathrm{E}-02$

1.2176

$4.5522 \mathrm{E}-03$

3.0372E-04

0.0

0.0

0.0

0.0

0.0

0.0

0.0

0.0

0.0

0.0

0.0

0.0

0.0

0.0

0.0

0.0

0.0

0.0

1.3779

0.0

.59791

$-1.4428 \Sigma+04$

0.0

0.0

0.0

0.0

1.0000

0.0

0.0

0.0

0.0

0.0

0.0

0.0

0.0

0.0

0.0

0.0

0.0

0.0

0.0

0.0

0.0

0.0

0.0

0.0

0.0

0.0

0.0

0.0

0.0

0.0

0.0

0.0 .

0.0

0.0

0.0

0.0

0.0

0.0 
HNF-2238

Revision 0

ESP $V-5.4$

STREAM: ap-102

6-Feb-98. RAGE 3

STREAM: ap-10I

POINT: 3

Temperature,

Pressure, atm

$\mathrm{pH}$

Total mol/hr

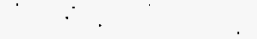

H2O
ALOH3
CAC2O4
CASO4
CO2
CSCL
CSNO3
FEIIIOH3
H3PO4
HNO2
KCL
NAF
NAHCO3
NANO3
NIC2O4

NIOH2

PBC204

PBNO22

EBO

SIO2

OHION

ALOH2ION

AIOH 4 ION

ALOHION

CAEION

CAHCOZION

CAION

CAOHION

CAPOAION

CIION

COSION

CR2O7ION

CRO4ION

Aqueous

12.000

1.0000

15.299 .

4584.2

$\mathrm{kg} / \mathrm{h} 5$

65.660

1.3440E- 09

$1.5885 \mathrm{E}-09$

$1.1346 \mathrm{E}-09$

$1.4785 \mathrm{E}-16$

6. $0049 \mathrm{z}-07$

$4.3549 \mathrm{E}-0.5$

2.2460E-12

3.5810E-29

$5.2809 \mathrm{E}-13$

$1.6295 \mathrm{E}-04$

.14910

$2.55025-07$

.14835

$9.9866 \mathrm{E}-16$

$1.1480 \mathrm{E}-08$

$3.1834 \mathrm{E}-18$

8.4063E-17

$3.0789 \mathrm{E}-09$

2. $3464 \mathrm{E}-12$

2.8818

1. $1584 \mathrm{E}-18$

2.2.404

$2.6354 \mathrm{E}-28$

$7.3188 \mathrm{E}-11$

$1.4998 \mathrm{E}-15$

4. $0405 \mathrm{E}-07$

2.8702E-06

$1.0997 \mathrm{E}-05$

.10182

1.0843

1. $4175 \mathrm{E}-21$

2. $6541 \mathrm{E}-02$

1. $1788 \mathrm{E}-04$

4. $1376 \mathrm{E}-07$

CSSOAION

EEIIIOH2ION

FEIIIOHAION

$8.9284 \mathrm{E}-22$

$1.7546 \mathrm{E}-06$

$5.6937 \mathrm{E}-32$

$6.9254 \mathrm{E}-02$

FION

H2PO4ION

H3SIO 4 ION

$7.9656 \mathrm{E}-15$

$2.1703 E-06$

$1.0750 \mathrm{E}-06$

HCOZION

HION

HOXAIATION

HP207ION

HPBO2ION

HPO4ION

IISO 4 ION

KION

$1.8782 \mathrm{E}-12$

$3.0897 \mathrm{E}-17$

$5.0876 \mathrm{E}-15$

9. $3584 \mathrm{E}-23$

$7.8238 \mathrm{E}-05$

$1.4340 \mathrm{E}-05$

$6.7716 \mathrm{E}-17$

1.9437

$1.8614 \mathrm{E}-02$

8.0254z-02

NA2FION

NACO3ION

1.3191

$\begin{array}{lll}\text { solid } & \text { Vapor } & \text { Organtc } \\ 12.000 & 1.000 & 12.000 \\ 1.0000 & 1.0000 & 1.0000\end{array}$

$12.705 \quad 0.0 \quad 0.0$

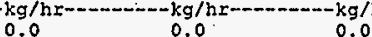

$\begin{array}{lll}.23690 & 0.0 & 0.0\end{array}$

0.0

0.0

0.0

0.0

0.0

0.0

0.0

0.0

0.0

0.0

0.0

0.0

0.0

$3.2465 E-04$

0.0

0.0

$0.0 \quad 0.0$

$0.0 \quad 0.0$

$0.0 \quad 0.0$

$0.0 \quad 0.0$

0.0

0.0

0.0

0.0

0.0

0.0

0.0

0.0

0.0

0.0

0.0

0.0

0.0

0.0

0.0

0.0

.0 .0

0.0

0.0

0.0

0.0

0.0

0.0

0.0

0.0

0.0

0.0

0.0

0.0

0.0

0.0

0.0

0.0

0.0

0.0

0.0

0.0

0.0

0.0

0.0

0.0

0.0

0.0

0.0

0.0

0.0

0.0

0.0

0.0

0.0 .

0.0

0.0

0.0

0.0

0.0 .

0.0

0.0

0.0

0.0

0.0

0.0

0.0

0.0

0.0

0.0

0.0

$\begin{array}{ll}0.0 & 0.0 \\ 0.0 & 0.0\end{array}$

0.0

0.0
0.0

0.0

0.0
0.0

0.0

0.0

0.0

0.0

0.0

0.0

0.0

0.0

0.0

0.0

0.0

0.0

0.0

0.0

0.0

0.0

0.0

0.0

0.0

0.0

0.0

0.0

0.0

0.0

0. 0

0.0

0.0

0.0

0.0

0.0

0.0

0.0

0.0

0.0

0.0

0.0

0.0

0.0

0.0

0.0

0.0 
HNF-2238

Revision 0

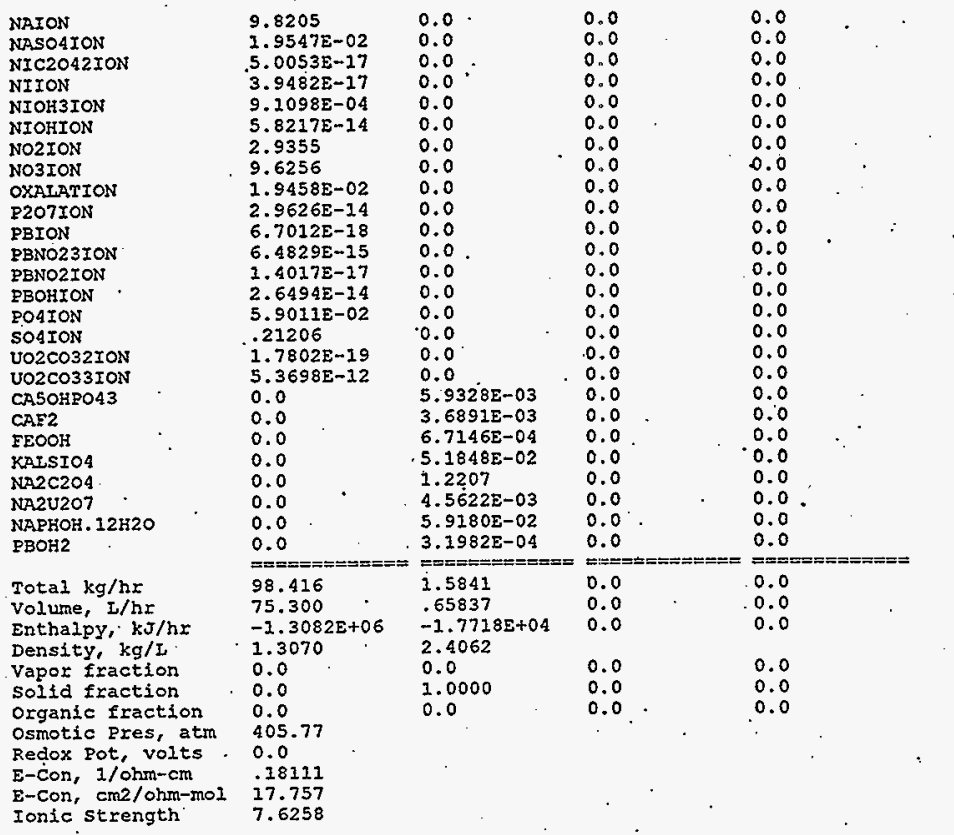


HNF-2238

Revision 0

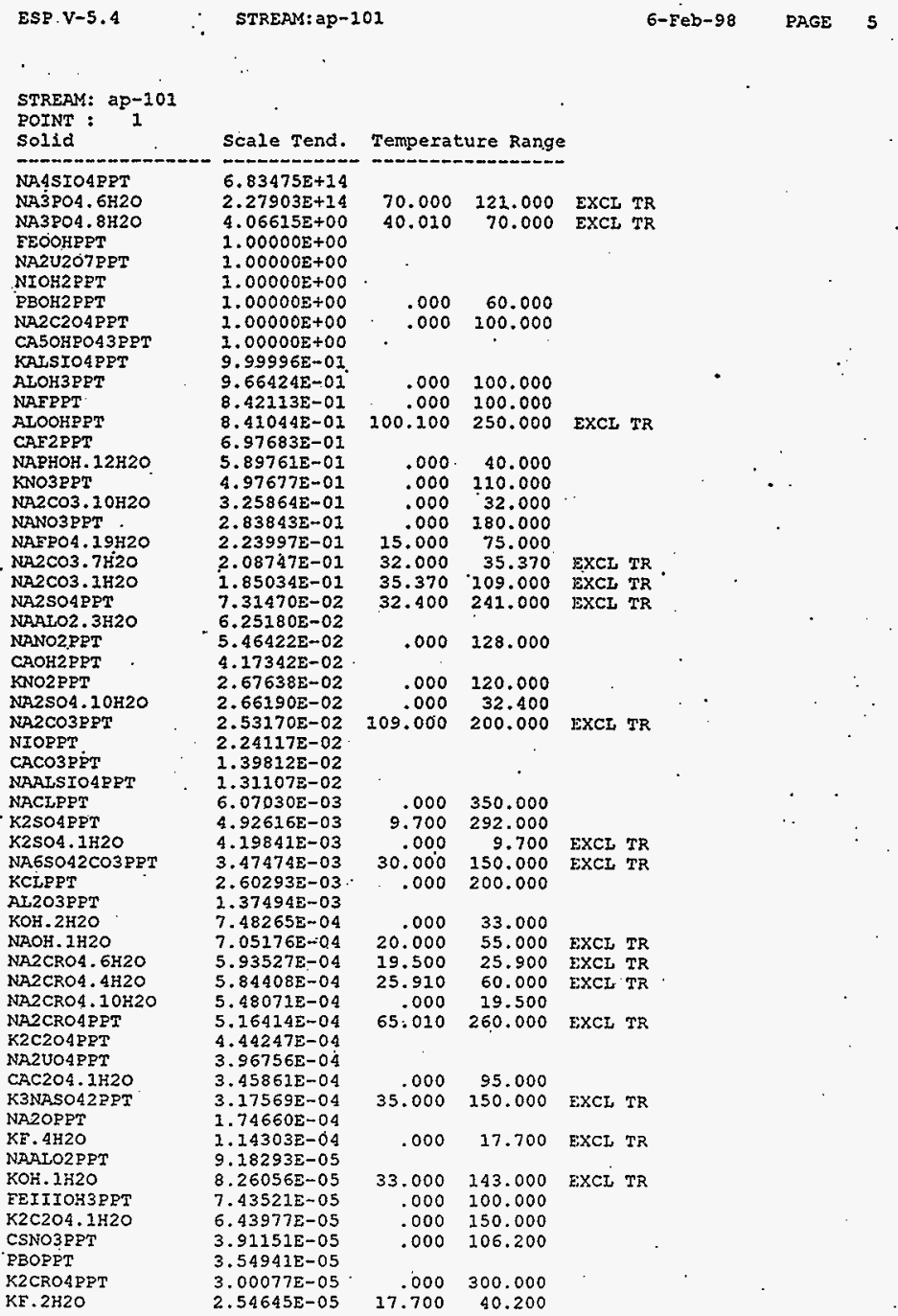


HNF-2238

Revision 0

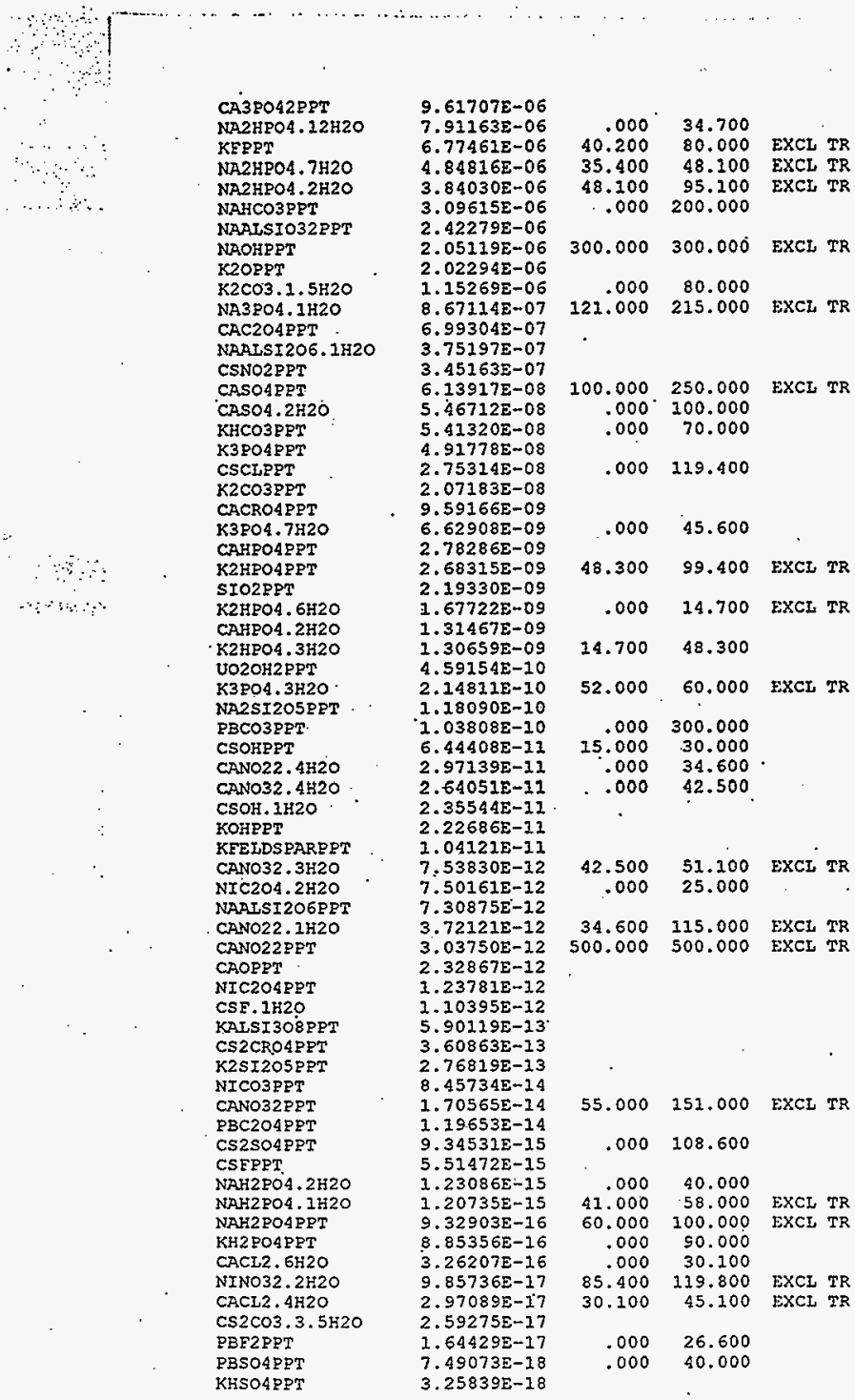


HNF-2238

Revision 0

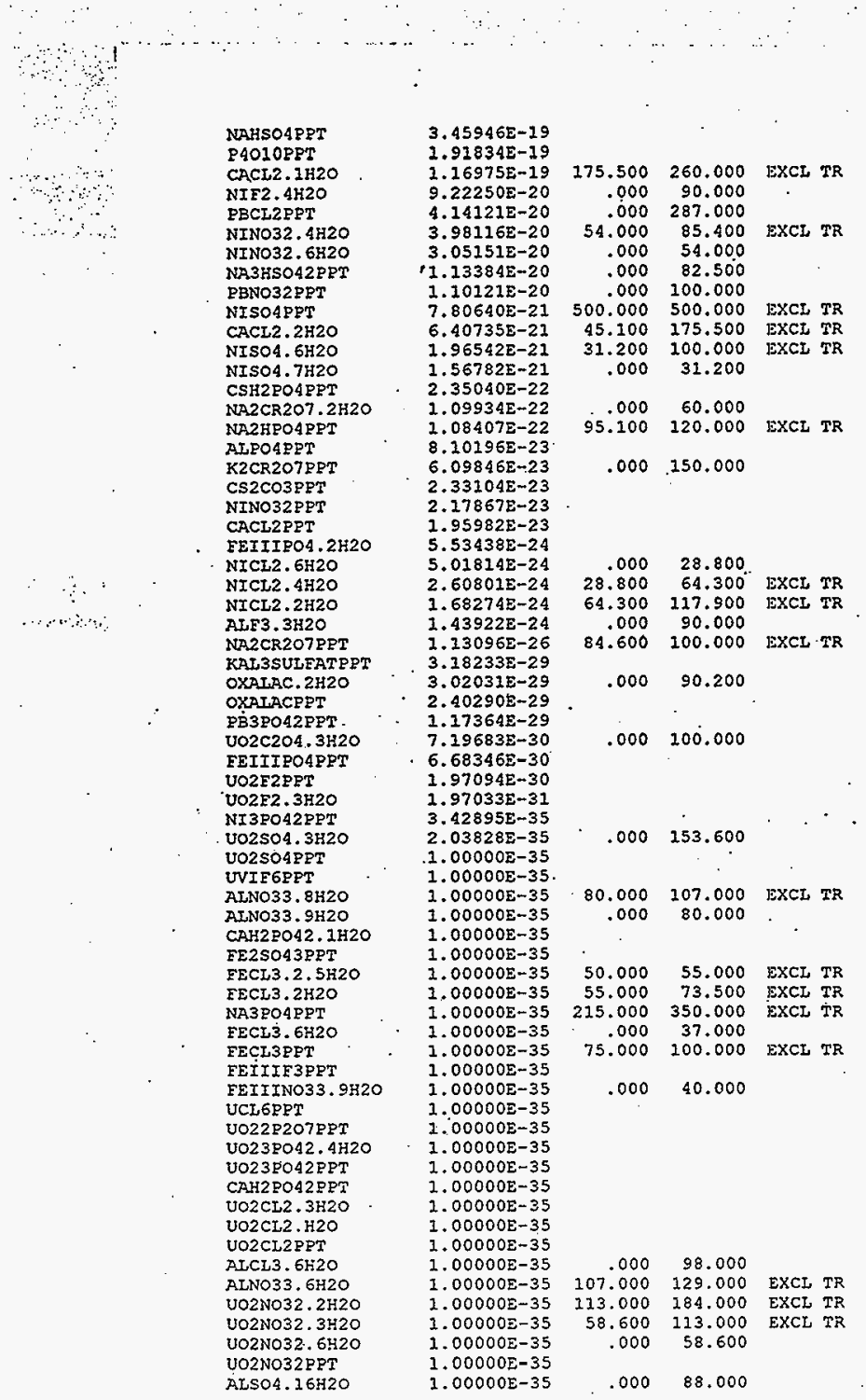


HNF-2238

Revision 0

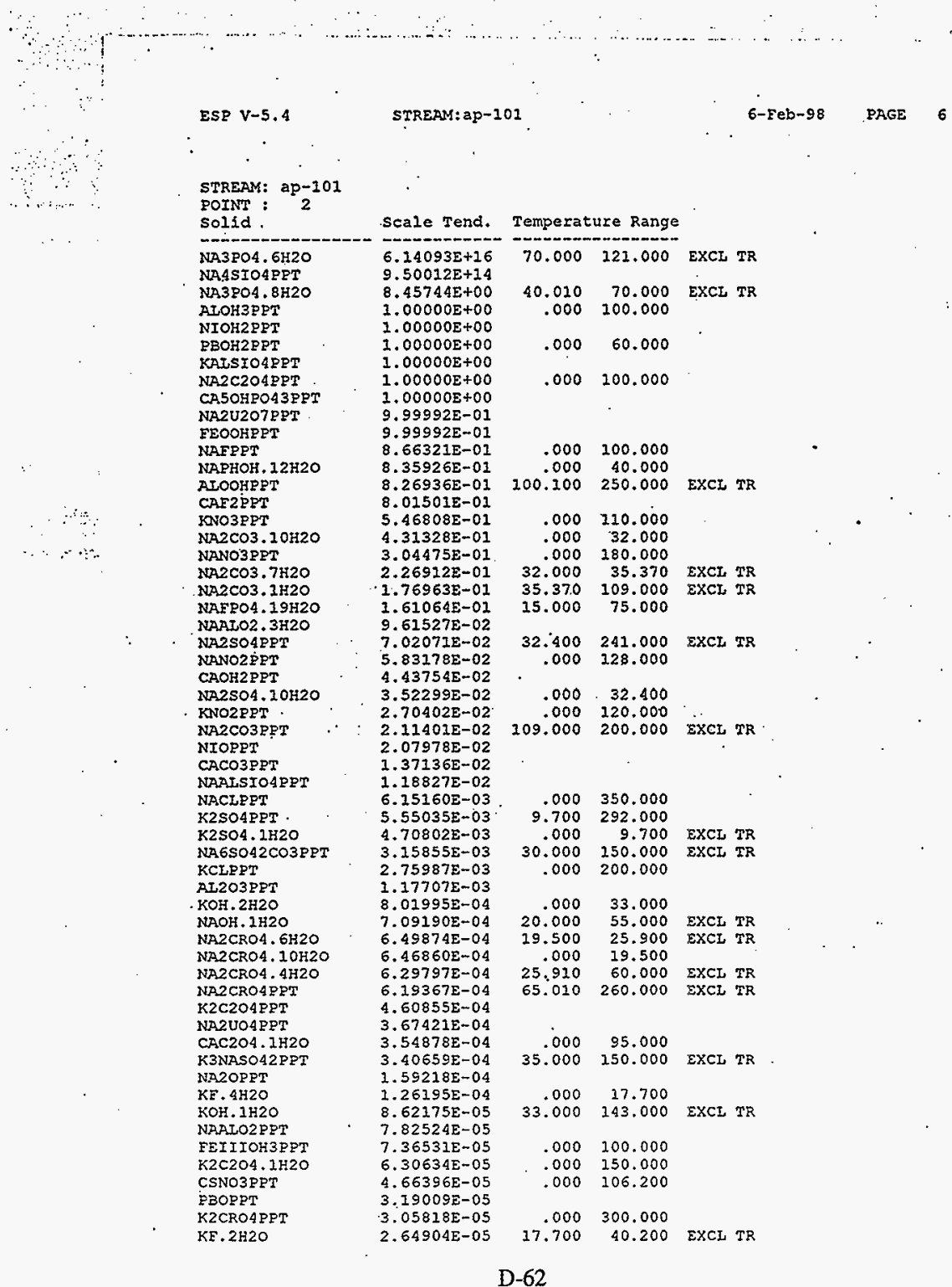


HNF-2238

Revision 0

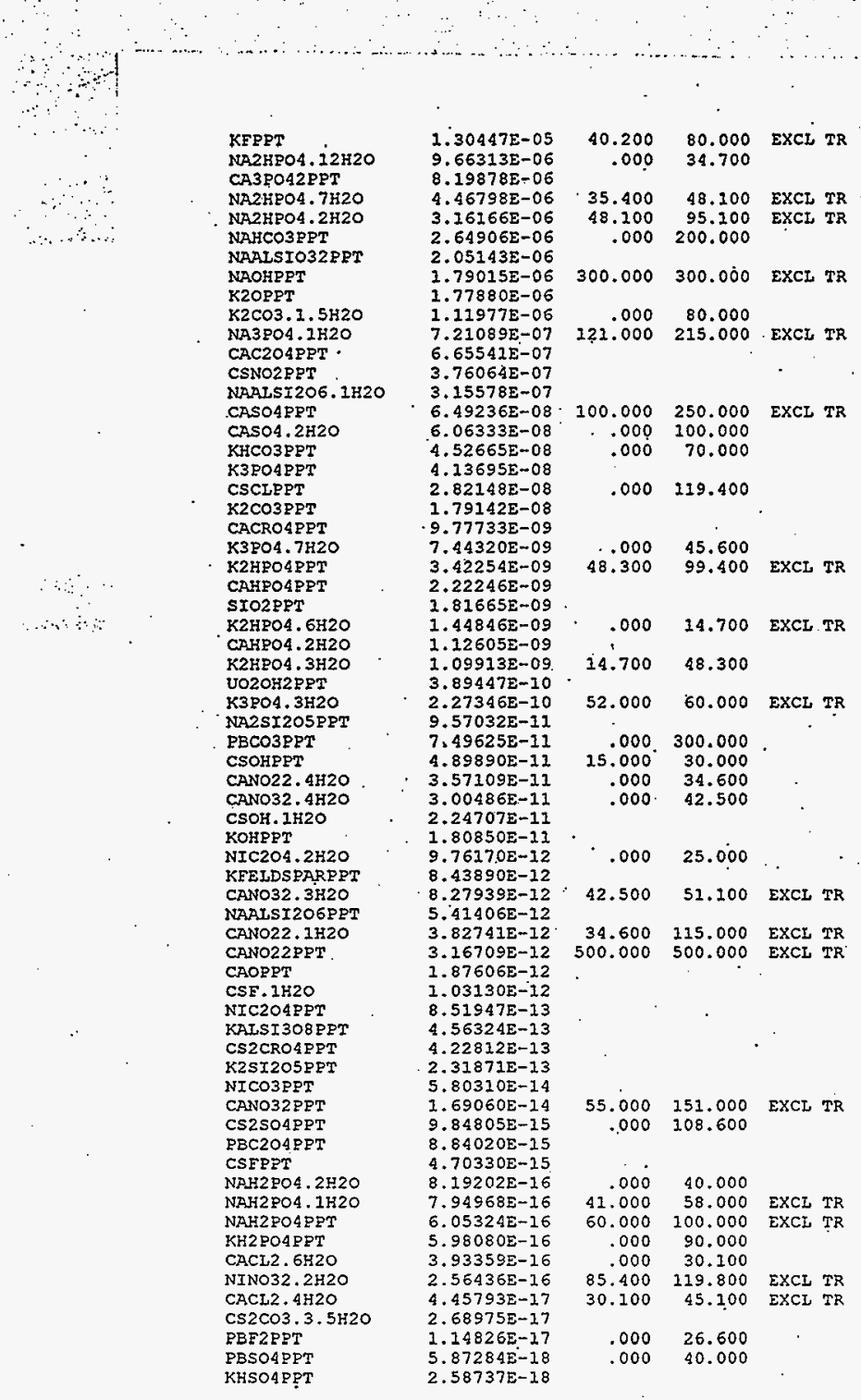


HNF-2238

Revision 0

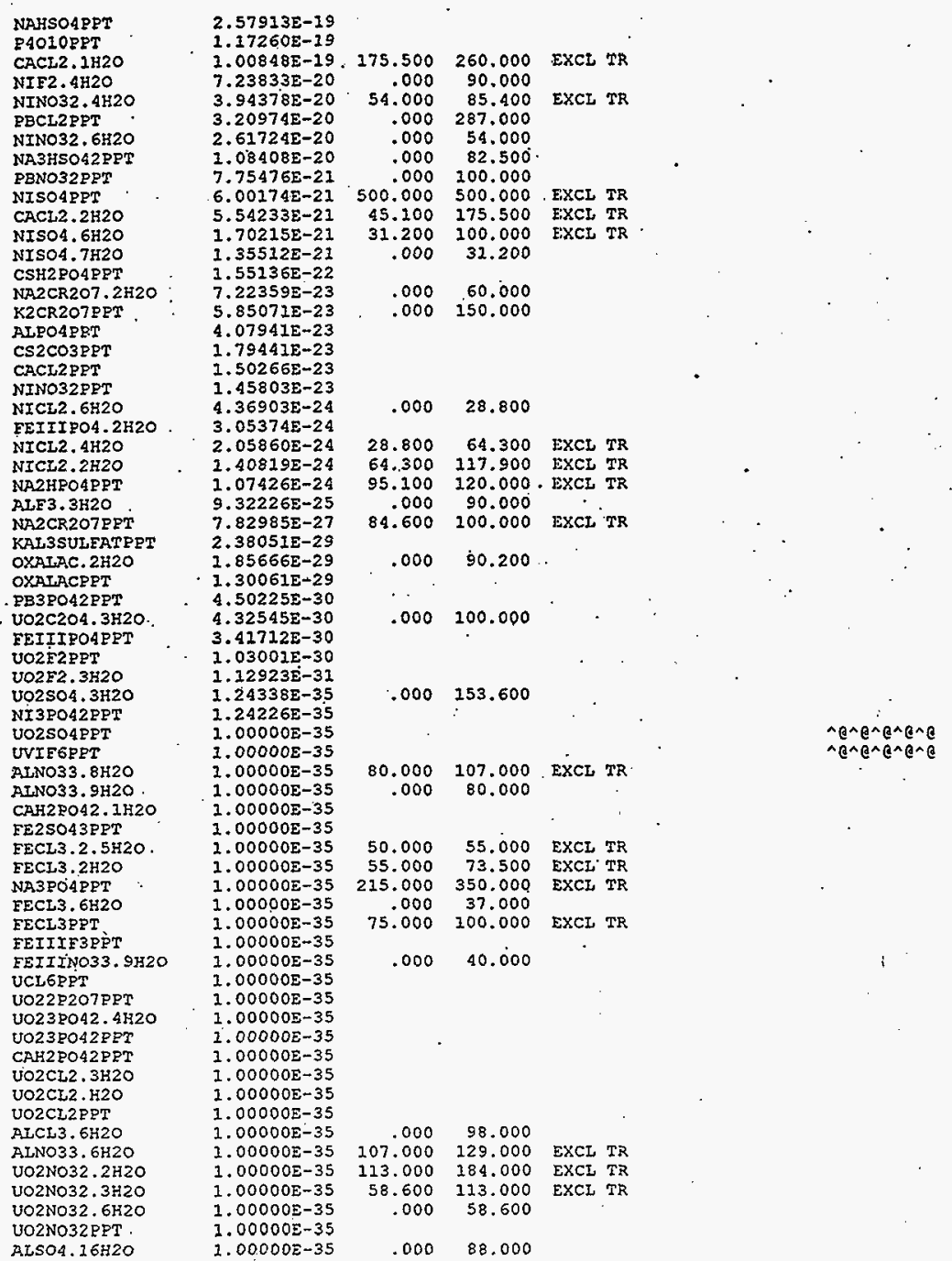


HNF-2238

Revision 0

ESP $\vee-5.4$

STREAM: ap-101

6-Feb-98 PAGE 7

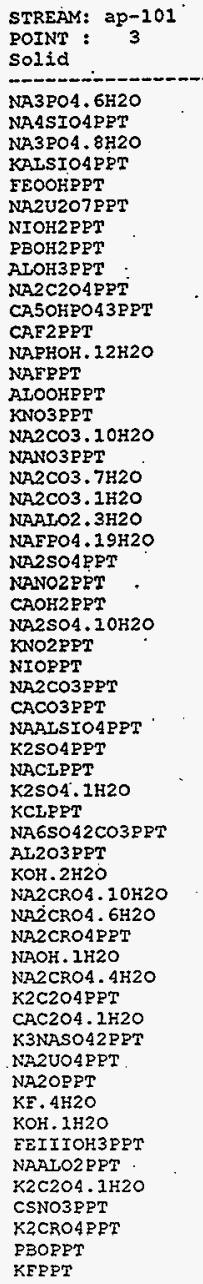

scalle Tend. Temperature, Range

$2.43036 \mathrm{E}+19 \quad 70.000 \quad 121.000$ EXCL TR

1. $40279 \mathrm{E}+15$

$1.52625 \mathrm{E}+01$

$1.00002 \mathrm{E}+00$

$1.00000 \mathrm{E}+00$

$1.000005+00$

1. $00000 \mathrm{E}+00$

1. $00000 \mathrm{E}+00$

1. $00000 \mathrm{E}+00$

$1.00000 \mathrm{E}+00$

$1.00000 \mathrm{E}+00$

1. $00000 \mathrm{E}+00$

9.99999E-01

8. $82565 \mathrm{E}-01$

7. $85616 \mathrm{E}-01$

$6.06806 \mathrm{E}-01$

5.74093E-01

$3.27493 \mathrm{E}-01$

2. 46451E-01

1. $68475 \mathrm{E}-01$

1. $49348 \mathrm{E}-01$

$6.96978 \mathrm{E}-02$

$6.74393 \mathrm{E}-02$

$6.23485 \mathrm{E}-02$

$5.28236 \mathrm{E}-02$

4. $80410 \mathrm{E}-02$

2. $73677 \mathrm{E}-02$

1. $92810 \mathrm{E}-02$

1. $74498 \mathrm{E}-02$

1. $48995 \mathrm{E}-02$

1. 074625-02

$6.29827 \mathrm{E}-03$

$6.24189 \mathrm{z}-03$

$5.28933 \mathrm{E}-03$

2. $93570 \mathrm{E}-03$

$2.89077 \mathrm{E}-03$

9. $38874 \mathrm{E}-04$

8. 61825E-04

$7.77443 \mathrm{E}-04$

7. $63747 \mathrm{E}-04$

7. $55031 \mathrm{E}-04$

$7.21646 \mathrm{E}-04$

$6.80948 \mathrm{E}-04$

4. $80697 \mathrm{E}-04$

4. $02965 \mathrm{E}-04$

$3.67845 \mathrm{E}-04$

3. $42252 \mathrm{E}-04$

1. $45047 \mathrm{E}-04$

1. $35015 \mathrm{E}-04$

9. 04249E-05

$7.29964 \mathrm{E}-05$

6. $47030 \mathrm{E}-05$

$6.15732 E-05$

$5.62365 \mathrm{E}-05$

$3.12442 \mathrm{E}-05$

2. $80495 E-05$

$$
40.01070 .000 \quad \text { EXCL TR }
$$

$.000 \quad 60.000$

$.000 \quad 100.000$

.000100 .000

100.11

$.000 \quad 40.000$

$.000 \quad 100.000$

$100.100 \quad 250.000$

. 000 . 110.000

$.000 \quad 32.000$

$.000 \quad 180.000$

$32.000 \quad 35.370$

$35.370 \quad 109.000$

$25.000 \quad 75.000$

$32.400 \quad 241.000$

.000128 .000

$.000 \quad 32.400$

$.000 \quad 220.000$

109.000

200.000

EXCL TR

$9.700 \quad 292.000$

.000350 .000

$.000 \quad 9.700$

$.000 \quad 200.000$

$30.000 \quad 150.000$

EXCL TR

EXCI TR

$\begin{array}{rrr}.000 & 33.000 \\ .000 & 19.500 \\ 19.500 & 25.900 \quad \text { EXCL, TR }\end{array}$

EXCL TR

$65.010 \quad 260.000$

$20.000 \quad 55.000$

$25.910 \quad 60.000$

EXCI TR

$.000 \quad 95.000$

$35.000 \quad 150.000$

EXCL TR

$.000 \quad 17.700$

$33.000 \cdot 143.000$

$.000 \quad 100.000$

EXCL TR

$.000 \quad 150.000$

$.000 \quad 106.200$

$.000 \quad 300.000$

$40.200 \quad 80.000$ EXCL TR 
HNF-2238

Revision 0

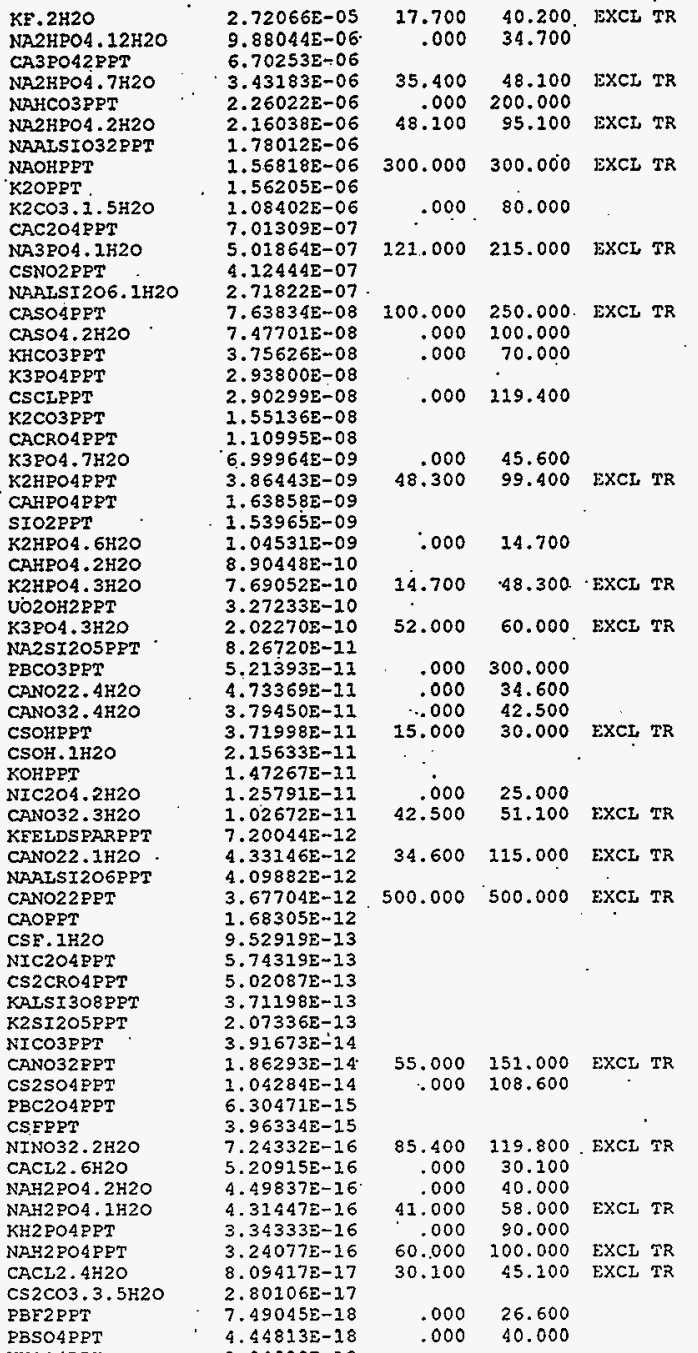


HNF-2238

Revision 0

NAHSOAPPT

CACL2 + $1 \mathrm{H} 2 \mathrm{O}$

P4OIOPPT

NIF2, $4 \mathrm{H} 20$

NINO32. 4 H2O

PBCL2PPM

NINO32.6H2O

NA3HSO42PPT .

CACL2. 2H2O

PBNO32PET

NISO4 PP2

NISO4. 6H2O

NISO4 $7 H 2 O$

CSH2PO4FRT

K2CR2OTEPI

NA2CR207. $2 \mathrm{H} 2 \mathrm{O}$

AIPO4PFT

CS2C03PPT

CACL2PPT

NINO32PFT

NICL2. $6 \mathrm{H} 2 \mathrm{O}$

NICL2 , 4H2O

EEIIIPO4 . 2H:20

NICL2. 2H2O

AIF $3.3 H 2 O$

NA2HPO 4 PPT

NA2CR207PPT

KAL3SULFATPPT

OXAIAC. $2 \mathrm{HzO}$

OXALACPET

UO2C2O4 . 3H2O

FEIIIPO4PPT

PB3PO42EPT

UO2E2PPI

บO2F2. 3H2O

ALCL3.6H2O

UO2504. $3 \mathrm{H} 20$

UO2SO4PPT

UVI E6PPT

ALNO33. $6 \mathrm{K2O}$

AISO4. 16420

AISO4 PRT

AINO33. 8H20

ALNO33.9H2O

CРH 2 PO42 . 1 H2O

NI3PO42PPT

FE2SO43EPT

EECL3.2.5H2O

EECL3.2H2O

NA3 PO4 PET

EECL3.61:20

EECL3PPT

UCL 6 PPT

U022P207PPT

VO23POA2 . 4 H2O

UO23PO42 PPT

FEIIIE3PTT

U०2 $2 \mathrm{Ct}$, $3 \mathrm{H} 2 \mathrm{O}$

UO2 $252 . H 2 O$

UO2CL 2 PP'T

FEIIINO33. SH2O

CAH2PO42RPT

UO2No32. $2 \mathrm{H} 2 \mathrm{O}$

บO 2 NO 32.3420

Vo2No32. 6420
1. $90913 E-19$

$9.62441 \Sigma-20$

$6.58178 \mathrm{E}-20$

$5.44954 \mathrm{E}-20$

$3.93926 \mathrm{E}-20$

$2.408145-20$

$2.21256 \mathrm{E}-20$

$9.89913 \mathrm{E}-21$

$5.30516 \mathrm{E}-21$

$5.29153 E-21$

4. $56162 \mathrm{E}-21$

1.45295E-2I

1. $15215 \mathrm{E}-21$

$8.47227 \mathrm{E}-23$

$5.56726 \mathrm{E}-23$

$4.68532 \mathrm{E}-23$

1. $62463 \mathrm{E}-23$

$1.38186 \mathrm{E}-23$

$1.27328 \mathrm{E}-23$

$9.63122 \mathrm{E}-24$

$3.69469 \mathrm{E}-24$

1. $59155 \mathrm{E}-24$

1. $37984 \mathrm{E}-24$

$1.16397 \mathrm{E}-24$

5. $49969 \mathrm{E}-25$

$6.12180 \mathrm{E}-27$

$5.44152 E-27$

1. $59041 \mathrm{E}-29$

$1.12226 \mathrm{E}-29$

$6.88090 \mathrm{E}-30$

. $2.517475-30$

$1.43062 \mathrm{E}-30$

$1.09602 \mathrm{E}-30$

$5.10824 \mathrm{E}-31$

$6.13987 \mathrm{E}-32$

1. $00000 \mathrm{E}-35$

$1.00000 \mathrm{E}-35$

1. $00000 \mathrm{E}-35$

$1.00000 \mathrm{E}-35$

$1.00000 \mathrm{E}-35$

$1.00000 \mathrm{E}-35$

$1.00000 \mathrm{E}-35$

$1.00000 \mathrm{E}-35$

$1.00000 \mathrm{E}-35$

1. $00000 \mathrm{E}-35$

$1.00000 \mathrm{E}-35$

1. $00000 \mathrm{E}-35$

3. $00000 \mathrm{E}-35$

1. $00000 \mathrm{E}-35$

$1.00000 \mathrm{E}-35$

$1.00000 \mathrm{E}-35$

$1.00000 \mathrm{E}-35$

$1.00000 \mathrm{E}-35$

$1.00000 \mathrm{E}-35$

$1.00000 \mathrm{E}-35$

I. $00000 \mathrm{E}-35$

1. $00000 \mathrm{E}-35$

1. $00000 \mathrm{E}-35$

$1.00000 \mathrm{E}-35$

$1.00000 \mathrm{E}-35$

1. $00000 \mathrm{E}-35$

1. $00000 \mathrm{E}-35$

$1.000002-35$

1.00000E-35

1. $00000 \mathrm{E}-35$
175.50

$$
.000
$$

54.000

.000

.000

.000

45.100 .000

500.000

31.200

.000

.000

.000

.000

28.800

$28.800 \quad 64.300$

$64.300 \quad 117.900$

.000

95.100

90.000

.84 .600

$$
120.000
$$

100.000

$$
\begin{array}{rr}
.000 & 90.200 \\
.000 \quad 100.000
\end{array}
$$

$.000 \quad 98.000$

$.000 \quad 153.600$

$107.000 \quad 129.000$ .000

88.000

$80.000 \quad 107.000$

$.000 \quad 80.000$

50.000

55.000

215.000

.000

75.000

73.500

350.000

37.000

100.000

EXCL TR

EXCI $T R$

EXCI TR

EXCL TR

EXCL TR

EXCL TR $\therefore$ anenanane

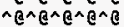

.000

113.000

58.600

.000
40.000

184.000

113.000

58.600
EXCL TR

EXCL TR 
HNF-2238

Revision 0

This page intentionally left blank. 
HNF-2238

Revision 0

\section{APPENDIX E}

\section{1-AW PROJECT REQUIREMENTS AND BASIS}


HNF-2238

Revision 0

This page intentionally left blank. 
HNF-2238

Revision 0

\section{APPENDIX E 241-AW PROJECT REQUIREMENTS AND BASIS}

\section{Purpose}

Initially, only the transfer system was included in the evaluation of the 241-AW Tank Farm. It is clear from the evaluation that some work scope is needed to address system deficiencies. In order to ensure those issues with all of the systems are addressed by any new work scope, all of the systems need to be evaluated. As part of the W-314 Project definition, other systems in the 241-AW Tank Farm were evaluated in fiscal year 1995 in a system condition assessment survey. A number of issues and system deficiencies were identified and documented during the system condition assessment survey. The purpose of this attachment is to present a preconceptual project scope for the 241-AW Tank Farm. A number of system deficiencies were identified in the course of this evaluation. Many of the deficiencies can be remedied by repair or replacement in kind. Only a few of the deficiencies will require new project work scope to remedy. The tables below identify issues that need to be addressed. Solutions for five issues should be included in new work scope, namely the AW-A and AW-B Valve Pit jumper modifications (see Table E-2a for the nozzle connections); the AW Stack modification necessary to provide National Emission Standards for Hazardous Air Pollutants (NESHAP) compliant continuous emissions monitoring; replacement of the transfer pump in tank 241-AW-104; connecting line LIQW-702 directly to line SN-220; and installation of flow meters in tank 241-AW-101 primary ventilation exhaust ducting. Preconceptual design work to address all of these issues is being developed. The other issues listed in the tables and in the body of this document need to be addressed and remedied. At the time of this writing however it is apparent that the best way to address most of these issues is through maintenance activities, negotiation with regulators, or other work scope which is not project related.

This list of issues was developed using the system condition assessment survey, and the evaluation of the transfer system done for this document. This issues list is intended to be modified over time as new issues are identified or clearer definition of requirements and basis becomes available. Systems included in this evaluation are: ventilation system, transfer system, electrical system, monitor and control system, utilities systems, and sampling systems. The attempt here is to identify issues (system inadequacies) with all of the systems, reference requirements not met by current system configuration, and state the basis for the requirement. Each of the systems has its own issues table. It is important to note that some of this work may already be included as project scope. Part of this evaluation is to determine the value added to combining all of the 241-AW Tank Farm system deficiencies into a single project. This evaluation will provide input to assist in making that decision. 
HNF-2238

Revision 0

\section{Ventilation system}

In assessing the tank ventilation system, Initial Assessment Report HVAC Systems, WHC-SD-W314-ES-022 (Kriskovich 1996) was used as the basis for the evaluation.

The performance requirements for the ventilation system are as follows: LCO 3.2.1 of TSR-006 requires that "the active primary ventilation system shall be operable." The electrical system repairs listed in the condition assessment item 1 in Table E-1 should be addressed by maintenance activities. The ventilation system will also need to meet environmental permit requirements. The stack upgrades listed in Item 2 in Table E-1 should be addressed by project work scope. Finally; Item 3 in Table E-1 requires further evaluation.

In addition to the issues listed in Table E-1 below, the Heating, Ventilation, and Air Conditioning (HVAC) system will need to be capable of removing heat added to the tank by operation of two mixer pumps. The HVAC system will need to remove sufficient heat such that the temperature of the waste does not exceed $90.6^{\circ} \mathrm{C}\left(195^{\circ} \mathrm{F}\right)$. Although the temperature of the waste is expected to rise during operation of the mixer pumps, the amount of time necessary to reach $90.6^{\circ} \mathrm{C}\left(195^{\circ} \mathrm{F}\right)$ is on the order of days. The time required to operate the mixer pumps to achieve the desired dissolution is expected to be on the order of hours (see Attachment 1). The temperature limitation of the waste is therefore not considered to be an issue even with operation of the mixer pumps. Mixer pump operation in tank 241-AW-101 will be modeled (hydraulic/chemical) to provide a basis for the required duration of mixer pump operation. The operations concept and procedures will need to be written to consider the mixer pump operation time limitations.

Table E-1. Ventilation System. (2 Sheets)

\begin{tabular}{|l|l|l|l|}
\hline \multicolumn{1}{|c|}{ Issue } & \multicolumn{1}{|c|}{ Process needs } & \multicolumn{1}{|c|}{ Basis } & $\begin{array}{l}\text { Suggested scope of work } \\
\text { to address issue }\end{array}$ \\
\hline $\begin{array}{l}\text { 1. Electrical supply and } \\
\text { control circuits to the } \\
\text { primary fans are in poor } \\
\text { condition and jeopardize } \\
\text { the reliability of the } \\
\text { system (wire terminations } \\
\text { are not properly supported } \\
\text { and control circuits are not } \\
\text { fully functional). }\end{array}$ & $\begin{array}{l}\text { Repair or replace } \\
\text { electrical components in } \\
\text { the HVAC system. } \\
\text { Specific components are } \\
\text { identified in the Condition } \\
\text { Assessment Survey (WHC- } \\
\text { SD-W314-ES-020). }\end{array}$ & $\begin{array}{l}\text { SCO 3.2.1 Technical Safety } \\
\text { that the active primary } \\
\text { ventilation system shall be } \\
\text { operable. The basis for this } \\
\text { requirement is the need to } \\
\text { prevent flammable gases } \\
\text { fom accumulating in the } \\
\text { tank headspace. }\end{array}$ & $\begin{array}{l}\text { Write a work package to } \\
\text { confirm condition of } \\
\text { electrical supply and } \\
\text { control circuits. } \\
\text { Document specific } \\
\text { components requiring } \\
\text { repair or replacement }\end{array}$ \\
\hline
\end{tabular}


HNF-2238

Revision 0

Table E-1. Ventilation System. (2 Sheets)

\begin{tabular}{|c|c|c|c|}
\hline Issue & Process needs & Basis & $\begin{array}{l}\text { Suggested scope of work } \\
\text { to address issue }\end{array}$ \\
\hline $\begin{array}{l}\text { 2. The } 241-A W \text { primary } \\
\text { ventilation stack will likely } \\
\text { be redesignated from a } \\
\text { minor to a major stack. } \\
\text { Permitting, monitoring, } \\
\text { and potentially treatment } \\
\text { requirements will change. }\end{array}$ & $\begin{array}{l}\text { Upgrade the } 241-\mathrm{AW} \\
\text { ventilation system to be } \\
\text { compliant with NESHAP. } \\
\text { This is likely to require } \\
\text { changes to the effluent } \\
\text { monitoring system. A fully } \\
\text { NESHAP - compliant } \\
\text { ventilation stack system } \\
\text { has been developed under } \\
\text { Project W-420. The } \\
\text { estimated project cost for } \\
\text { a single stack is } \\
\text { approximately } \$ 200,000 \text {. }\end{array}$ & $\begin{array}{l}\text { A criterion for designation of } \\
\text { an effluent discharge stack as } \\
\text { minor or major is whether } \\
\text { the potential for unabated } \\
\text { discharges results in an off- } \\
\text { site exposure of } \\
1 \times 10^{-3} \mathrm{mSv} / \mathrm{yr} \\
(0.1 \mathrm{mrem} / \mathrm{yr}) \text {. Air modeling } \\
\text { requirements have recently } \\
\text { changed by a factor of } 1.5 \text {. } \\
\text { This increased factor causes } \\
\text { the current } 241-\mathrm{AW} \text { primary } \\
\text { stack to exceed } \\
6 \times 10^{-4} \mathrm{mSv} / \mathrm{yr} \\
(0.06 \mathrm{mrem} / \mathrm{yr}) \text { unabated } \\
\text { releases under current } \\
\text { conditions. Increased releases } \\
\text { are likely due to mixer pump } \\
\text { operation. }\end{array}$ & $\begin{array}{l}\text { This is project work } \\
\text { scope. The cesign for } \\
\text { changes to the } 241-\mathrm{AW} \\
\text { primary ventilation stack } \\
\text { will be similar to the } \\
\text { design done for the } \\
\text { W-420 Project (see } \\
\text { Attachment 2). }\end{array}$ \\
\hline $\begin{array}{l}\text { 3. The HVAC system } \\
\text { does not have the } \\
\text { capability of removing } \\
\text { toxic pollutants from off- } \\
\text { gas. This issue is } \\
\text { particularly important } \\
\text { during a Gas Release- } \\
\text { Event (GRE). The } \\
\text { concentrations of volatile } \\
\text { toxic pollutants in the tank } \\
\text { waste are not well } \\
\text { characterized. Release } \\
\text { limits are not currently } \\
\text { defined. }\end{array}$ & $\begin{array}{l}\text { Install or provide } \\
\text { expansion capability for an } \\
\text { activated carbon filter and } \\
\text { dry scrubber system in the } \\
\text { HVAC sysiem to reduce } \\
\text { or eliminate toxic } \\
\text { pollutants from the off-gas } \\
\text { stream. }\end{array}$ & $\begin{array}{l}\text { Toxic constituents are present } \\
\text { in off-gas from the tanks } \\
\text { potentially at concentrations } \\
\text { which will require abatement. } \\
\text { This follows the } \\
\text { recommendation made in } \\
\text { WHC-SD- } \\
\text { W314-ES-022. }\end{array}$ & $\begin{array}{l}\text { Adding the capability of } \\
\text { toxic constituent removal } \\
\text { to the HVAC systems is } \\
\text { currently planned for } \\
\text { W-314. Development of } \\
\text { a basis for this } \\
\text { requirement will require } \\
\text { vapor space sampling of } \\
\text { target Double-shell tanks } \\
\text { (DSTs) with analysis for } \\
\text { Tanks Advisory Panel } \\
\text { (TAPs). }\end{array}$ \\
\hline $\begin{array}{l}\text { 4. There currently is no } \\
\text { direct method to measure } \\
\text { the primary ventilation } \\
\text { airflow from tank } \\
241-\mathrm{AW}-101 \text {. The filtered } \\
\text { inlet air flowrate is } \\
\text { monitored but this doesn't } \\
\text { account for inleakage } \\
\text { through pump pits, etc. }\end{array}$ & $\begin{array}{l}\text { Install flow meters in tank } \\
241 \text {-AW-101 primary } \\
\text { ventilation exhaust } \\
\text { ducting. See Double-Shell } \\
\text { Tank Primary Ventilation } \\
\text { Exhaust Flow Monitor } \\
\text { System Design } \\
\text { Description, HNF-SD- } \\
\text { WM-SDD-074 for } \\
\text { conceptual design } \\
\text { information. }\end{array}$ & $\begin{array}{l}\text { Flow monitoring in } \\
\text { conjunction with hydrogen } \\
\text { monitoring equipment } \\
\text { aiready installed, will allow } \\
\text { for determination of the } \\
\text { overall hydrogen generation } \\
\text { and release rates. This } \\
\text { information will be necessary } \\
\text { for controlling the waste } \\
\text { degassing operation. See } \\
\text { Technical Basis for } \\
\text { Installation of the Double- } \\
\text { Shell Tank Exhaust Flow } \\
\text { Monitoring Systems, HNF- } \\
\text { SD-WM-ER-629 for a } \\
\text { detailed basis. }\end{array}$ & $\begin{array}{l}\text { Issue work package to } \\
\text { install flow monitoring } \\
\text { system on the } \\
241-\mathrm{AW}-101 \text { tank } \\
\text { exhaust header and } \\
\text { connect the output } \\
\text { signals to TMACS. }\end{array}$ \\
\hline
\end{tabular}


HNF-2238

Revision 0

\section{Transfer System}

The evaluation of the transfer system is documented in this report. The Tank Waste Remediation System Operation and Utilization Plan (TWRSO\&UP) (Kirkbride et al. 1997) transfer list was used as the starting point. All of the transfers originating or ending in 241-AW were extracted from the transfer list, and paths were defined for each of the different tank to tank transfers. All of the transfer equipment in the different transfer paths was considered.

Issue 1 in Table E-2 is the broken transfer pump in tank 241-AW-104. Although repair or replacement of a transfer pump is a major effort, the work is replacement in kind (most likely with an existing spare). It is recommended that if used the existing spare transfer pump should be shortened. An existing turbine pump such as the spare for the 241-AW-104 transfer pump can be shortened by replacing one or more shaft section(s) and transfer pipe section(s) with shorter pieces. These shorter pieces are available from the pump manufacturer. Even given the pump shortening, this work scope should be considered as replacement in kind.

Issue 2 and Issue 6 in Table E-2 are the jumper modifications needed in the AW-A and AW-B Valve Pits, and the 102-A central Pump Pit. Table E-2a lists the nozzles in each pits that need to be connected to support the identified transfers. A preconceptual design for the jumper configurations in each pit is included in Figure 1. Figure 1a shows an alternate manifold system that may add flexibility by including the 2 -in. lines. Figure 2 shows the jumper recommended in the 241-AW-102 02A central pump pit. Figure 3 shows the recommended jumper configuration for the 241-AW-103 and -106 tanks and for the 241-AN-106 tank. Issues 4 and 5 establish requirements that new jumper designs will need to meet. These issues will be addressed during the conceptual design of the jumper system.

Issue 3 in Table E-2 requires a field evaluation to determine what if any damage was done to the piping, valve pits, and monitoring equipment in the valve pit. The field evaluation should pressure test the transfer lines between the AW-A and AW-B valve pits and should visually inspect the valve pits for any signs of damage caused by acid leakage.

Several potential issues were evaluated and determined not to constitute significant problems; what follows is a brief discussion of those potential issues.

In December of 1984, a submersible pump was being used to transfer waste from tank 241-AW-101. The discharge line from the pump (5.1 $\times 10^{2} \mathrm{~m}$ [2-in.] rubber hose) had come loose from the discharge flange. The weight of the line caused the pump to rotate on its support cables and prevented the pump from being withdrawn from the tank. Support cables to the pump were cut and the pump was allowed to fall back into the tank. The pump is currently resting on the bottom of the tank. The potential issue is the possibility that the pump could either block the mixer or transfer pump intake or be thrown into the tank wall or instrumentation by the mixer pump outflow. A thrown transfer pump has the potential to damage an instrument tree, air lift circulator, or otherwise damage the tank structure. 
HNF-2238

Revision 0

Noting that this issue is not limited to a transfer pump in tank 241-AW-101 is important, other (perhaps all) tanks contain debris that could affect mixer or transfer pump operation. Debris in tank 241-SY-101 was addressed in the Los Alamos National Laboratory Safety Assessment (LANL) for the 241-SY-101 Mixer Pump, LA-UR-92-3196, Appendix D. The conclusions of the analysis were that none of the debris in tank 241-SY-101 is a credible threat to the structural integrity of the tank; that no permanent damage to instrument trees was anticipated to occur; and that entrapment of debris by the mixer pump was unlikely to occur. Operation of the mixer pump in tank 241-SY-101 has occurred without such an incident occurring since 1993. The mixer pump design and the transfer pumps have a screened intake that would likely prevent ingestion of debris. The safety assessment determined that this potential issue is not a significant problem.

Another potential issue is the Net Positive Suction Head (NPSH) required to operate the mixer and transfer pumps versus the NPSH available from the system. If the NPSH required is greater than the NPSH available, the pump will experience a cavitation on the suction side of the pump. A cavitation on either suction or discharge will lead to pump damage and early failure. In an evaluation by M.A. Przybylski, it was determined that the mixer pumps can be operated at full speed with a submergence (level difference between the impellers and the liquid level) of approximately $5.5 \mathrm{~m}$ (18 ft) (dependant upon the temperature of the liquid, $5.5 \mathrm{~m}[18 \mathrm{ft}]$ at $100^{\circ} \mathrm{C}\left[212^{\circ} \mathrm{F}\right], 1.2 \mathrm{~m}[4 \mathrm{ft}]$ at $\left.60^{\circ} \mathrm{C}\left[140^{\circ} \mathrm{F}\right]\right)$. The mixer pump can be run at slow speed ( 58 percent of full speed) with a submergence of $1.1 \mathrm{~m}(3.5 \mathrm{ft})$ for a waste temperature of $88^{\circ} \mathrm{C}\left(190^{\circ} \mathrm{F}\right)$.

Drawing the waste in the tanks down to $0.25 \mathrm{~m}$ (10-in.) is desirable. The NPSH required by the transfer pump (W-211 Sulzer Pump) is $5.5 \mathrm{~m}$ (18 ft). With the impeller located approximately $0.69 \mathrm{~m}(27-\mathrm{in}$.) from the bottom of the tank, the waste can be drawn down to $0.25 \mathrm{~m}\left(10\right.$-in.) provided the temperature of the waste is less than $60^{\circ} \mathrm{C}\left(140^{\circ} \mathrm{F}\right)$. At a temperature of $91^{\circ} \mathrm{C}\left(195^{\circ} \mathrm{F}\right)$, the submergence of the pump will need to be $3.4 \mathrm{~m}(11 \mathrm{ft})$ based on a SpG of 1.41 to prevent a cavitation. These calculations are included as Attachment 3. The temperature dependance of the pump submergence requirement will need to be factored into operating procedures to be developed for the tank retrieval activities. 
HNF-2238

Revision 0

Table E-2. Transfer System. (2 Sheets)

\begin{tabular}{|c|c|c|c|}
\hline Issue & Process needs & Basis & $\begin{array}{l}\text { Suggested scope of } \\
\text { work to address issue }\end{array}$ \\
\hline $\begin{array}{l}\text { 1. Tank 241-AW-104 } \\
\text { does not have a functional } \\
\text { transfer pump (the existing } \\
\text { pump has a broken } \\
\text { coupling in the shaft). }\end{array}$ & $\begin{array}{l}\text { Install a new or spare } \\
\text { pump into the Tank 241- } \\
\text { AW-104 Central Pump Pit } \\
\text { 04-A to provide the means } \\
\text { to transfer waste from the } \\
\text { tank. }\end{array}$ & $\begin{array}{l}\text { The TWRSO\&UP has } \\
\text { identified two supernate } \\
\text { transfers of } 2000 \mathrm{~m}^{3} \\
\left(540,000 \text { gal) and } 620 \mathrm{~m}^{3}\right. \\
\text { (164,000 gal) scheduled to } \\
\text { occur in October of } 1998 \text {. As } \\
\text { part of changes made during } \\
\text { the readiness to proceed } \\
\text { effort, tank } 241-\mathrm{AW}-104 \text { is } \\
\text { currently scheduled to be a } \\
\text { waste feed delivery tank with } \\
\text { transfers to } 241-\mathrm{AP}-102 \text { and } \\
241-\mathrm{AP}-104 \text { in June } 2004 \\
\text { (batch 5). The Waste Feed } \\
\text { Delivery (WFD) transfer will } \\
\text { be supernate only. }\end{array}$ & $\begin{array}{l}\text { Although the pump } \\
\text { will likely be replaced } \\
\text { with an existing spare, } \\
\text { the pump replacement } \\
\text { should be project work } \\
\text { scope due to the cost } \\
\text { and complexity } \\
\text { involved with the } \\
\text { removal and disposal } \\
\text { of the existing pump. }\end{array}$ \\
\hline $\begin{array}{l}\text { 1.a) Piping from pump to } \\
\text { the pit wall should use } \\
\text { existing pipe nozzles and } \\
\text { jumpers to the extent } \\
\text { possible. }\end{array}$ & $\begin{array}{l}\text { The pump shall connect to } \\
\text { wail nozzle } A \text {, and tank } \\
\text { return nozzle } G \text { in the } \\
\text { 241-AW-04A Central } \\
\text { Pump Pit. } \\
\text {. }\end{array}$ & $\begin{array}{l}\text { Use of Existing piping systems } \\
\text { is required to reduce costs, to } \\
\text { minimize disruption of } \\
\text { operations, and to eliminate } \\
\text { the need of excavation in the } \\
\text { tank farms (Reference } \\
\text { Drawing \# H-14-020802 sh.3, } \\
\text { for existing piping system). }\end{array}$ & . \\
\hline $\begin{array}{l}\text { 1.b) The existing pump (if } \\
\text { functional) would not meet } \\
\text { system flow and head } \\
\text { requirements. }\end{array}$ & $\begin{array}{l}\text { The pump shall provide a } \\
\text { minimum flow of } \\
0.53 \mathrm{~m}^{3} / \mathrm{min} \\
(140 \mathrm{gal} / \mathrm{min}) \text {, and a } \\
\text { maximum head of } 116 \mathrm{~m} \\
(379 \mathrm{ft}) .\end{array}$ & $\begin{array}{l}\text { The lowest pressure rating in } \\
\text { the. piping systems is } 1.6 \mathrm{MPa} \\
\text { ( } 230 \text { psi) in the } 3 \text {-in. SN-220 } \\
\text { line. A specific gravity of } 1.41 \\
\text { and a maximum allowable } \\
\text { operating pressure of } 1.6 \mathrm{MPa} \\
\text { (230 psi) suggest that the } \\
\text { maximum allowable dead head } \\
\text { pressure is } 116 \mathrm{~m} \text { ( } 379 \mathrm{ft}) . \\
\text { The minimum flow rate } \\
\text { requirement is based on } \\
\text { transferring } \\
3790 \mathrm{~m}^{3} \text { (1,000,000 gal) over } \\
\text { a five day period (Best } \\
\text { Engineering Judgement) } \\
\text { (calculations shown in } \\
\text { Attachment } 4 \text { ). }\end{array}$ & $\begin{array}{l}\text { The basis for the } \\
\text { flowrate will require } \\
\text { that a definition and } \\
\text { flow requirements for } \\
\text { "supernate" transfers } \\
\text { be developed. } \\
\text { Awaiting work plan } \\
\text { and cost estimate from } \\
\text { Pacific Northwest } \\
\text { National Laboratory } \\
\text { (PNNL). }\end{array}$ \\
\hline $\begin{array}{l}\text { 1.c) The existing pump in } \\
\text { tank } 241-\mathrm{AW}-104 \text { (a stick } \\
\text { type pump ) intake is } \\
\text { approximately } 2.5 \mathrm{~m} \\
\text { (100-in.) below the } \\
\text { nominal sludge/liquid } \\
\text { interface. }\end{array}$ & $\begin{array}{l}\text { Install a supernate pump } \\
\text { (flex and float or a } \\
\text { shorter stick-type pump), } \\
\text { such that the pump intake } \\
\text { is above the sludge level } \\
\text { in the tank. }\end{array}$ & $\begin{array}{l}\text { All of the transfers currently } \\
\text { identified from tank } 241-\mathrm{AW} \text { - } \\
104 \text { are supernate only. }\end{array}$ & \\
\hline
\end{tabular}


HNF-2238

Revision 0

Table E-2. Transfer System. (2 Sheets)

\begin{tabular}{|c|c|c|c|}
\hline Issue & Process needs & Basis & $\begin{array}{l}\text { Suggested scope of } \\
\text { work to address issue }\end{array}$ \\
\hline $\begin{array}{l}\text { 2. The current valve pit } \\
\text { jumper configuration will } \\
\text { not support the planned } \\
\text { transfers. }\end{array}$ & $\begin{array}{l}\text { The system shall be } \\
\text { designed such that the } \\
\text { nozzles identified in the } \\
\text { Table E-2a below can be } \\
\text { connected. }\end{array}$ & $\begin{array}{l}\text { Transfers identified in the } \\
\text { TWRSO\&UP and updated to } \\
\text { reflect the readiness to } \\
\text { proceed baselines, routes } \\
\text { identified in Appendix A. }\end{array}$ & $\begin{array}{l}\text { Installation of a new } \\
\text { jumper system is } \\
\text { project work scope. A } \\
\text { manifold sketch is } \\
\text { included as Figure } 1 . \\
\text { Fluor Daniel } \\
\text { Northwest to develop } \\
\text { detailed drawings. }\end{array}$ \\
\hline $\begin{array}{l}\text { 3. In } 1984 \text { or } 1985 \text {, an } \\
\text { acid transfer from PUREX } \\
\text { damaged 2-in. SL-161 and } \\
\text { potentially other } \\
\text { equipment. }\end{array}$ & $\begin{array}{l}\text { The AW-A and AW-B } \\
\text { Valve Pits and transfer } \\
\text { lines between these valve } \\
\text { pits shall be evaluated } \\
\text { (pressure tested). } \\
\text { Damaged equipment shall } \\
\text { be repaired or replaced. }\end{array}$ & $\begin{array}{l}\text { Existing equipment will need } \\
\text { to be maintained or repaired to } \\
\text { support continued operations } \\
\text { through Phase } 1 \text { privatization. } \\
\text { Additionally, equipment will } \\
\text { also likely be used through the } \\
\text { end of Phase } 2 \text { privatization. }\end{array}$ & $\begin{array}{l}\text { Develop a work } \\
\text { package to visually } \\
\text { inspect the condition } \\
\text { of the } A W-A \text { and } A W- \\
B \text { Valve Pits and to } \\
\text { pressure test the SN- } \\
271 \text { line between the } \\
\text { two Valve Pits. }\end{array}$ \\
\hline $\begin{array}{l}\text { 4. Pump and Valve Pit } \\
\text { modifications need to be } \\
\text { designed so that they are } \\
\text { controlled by existing and } \\
\text { planned equipment. }\end{array}$ & $\begin{array}{l}\text { System shall be integrated } \\
\text { with both existing control } \\
\text { system and system to be } \\
\text { installed by Projects W- } \\
314 \text { and W-211. }\end{array}$ & $\begin{array}{l}\text { The current control strategy is } \\
\text { to control transfers from a } \\
\text { central control room (valve } \\
\text { positions shown on read out } \\
\text { board). }\end{array}$ & $\begin{array}{l}\text { Specific I\&C } \\
\text { integration } \\
\text { tequirements will be } \\
\text { developed by Vista } \\
\text { Research. }\end{array}$ \\
\hline $\begin{array}{l}\text { 5. Systems need to meet } \\
\text { RCRA (dangerous waste } \\
\text { rules) }\end{array}$ & $\begin{array}{l}\text { Pipe systems shail have } \\
\text { leak detection and shall be } \\
\text { self draining. }\end{array}$ & $\begin{array}{l}\text { The system needs to comply } \\
\text { with WAC-173-303. }\end{array}$ & $\begin{array}{l}\text { Compliance issues are } \\
\text { being negotiated with } \\
\text { regulators. }\end{array}$ \\
\hline $\begin{array}{l}\text { 6. The current system } \\
\text { only allows transfers out } \\
\text { of } 241-\mathrm{AW}-102 \text { to the } \\
242-\mathrm{A} \text { evaporator but the } \\
\text { capability is required to } \\
\text { also pump from } \\
241-\mathrm{AW}-102 \text { to } \\
241-\mathrm{AW}-106 \text {. }\end{array}$ & $\begin{array}{l}\text { Provide a jumper } \\
\text { connection from nozzle } K \\
\text { to nozzle } A \text { in the } 02-A \\
\text { central pump pit. Retain } \\
\text { connection from nozzle K } \\
\text { to nozzle G. See Figure } \\
2 \text {. }\end{array}$ & $\begin{array}{l}\text { To make the transfers listed in } \\
\text { the TWRSO\&UP, } \\
\text { (241-AW-102 to } 241-A W-106 \text { ) } \\
\text { a routing through the 02-A } \\
\text { central pump pit is needed. }\end{array}$ & $\begin{array}{l}\text { Installation of a new } \\
\text { jumper system is } \\
\text { project work scope. } \\
\text { Fluor Daniel } \\
\text { Northwest to develop } \\
\text { detailed drawings. }\end{array}$ \\
\hline $\begin{array}{l}\text { 7. The proposed new W- } \\
314 \text { line does not require } \\
\text { the use of Valve Pit } 241- \\
\text { A-A excepting waste } \\
\text { transferred from 204-AR. } \\
\text { A route from 204-AR to } \\
241-A W \text { that bypasses } \\
\text { Valve Pit } 241-A-A \text { would } \\
\text { allow this valve pit to be } \\
\text { abandoned. }\end{array}$ & $\begin{array}{l}\text { Connect line LIQW-702 } \\
\text { directly to line SN- } 220 \text {, } \\
\text { bypassing the } \\
241-A-A \text { Vaive Pit. }\end{array}$ & $\begin{array}{l}\text { Line LIQW-702 is required for } \\
\text { receipt of misc. Waste into the } \\
\text { DST system via the 204-AR } \\
\text { vault. Bypassing the } 241-\mathrm{A}-\mathrm{A} \\
\text { Valve Pit allows it to be } \\
\text { abandoned. }\end{array}$ & $\begin{array}{l}\text { Fluor Daniel } \\
\text { Northwest to develop } \\
\text { detailed drawings. }\end{array}$ \\
\hline
\end{tabular}


HNF-2238

Revision 0

Table E-2a. Valve Pit connections and dates.

\begin{tabular}{|l|l|l|l|}
\hline \multicolumn{1}{|c|}{$\begin{array}{c}\text { Valve pit 241-AW-A } \\
\text { connections }\end{array}$} & \multicolumn{1}{|c|}{ Need dates } & \multicolumn{1}{|c|}{$\begin{array}{c}\text { Valve pit 241-AW-B } \\
\text { connections }\end{array}$} & \multicolumn{1}{|c|}{ Need dates } \\
\hline nozzles L-1 to L-2 & $6 / 15 / 98-4 / 4 / 11$ (exists) & nozzles R-1 to R-14 & $10 / 10 / 98-12 / 1 / 10$ \\
\hline nozzles L-1 to L-14 & $8 / 6 / 98-$ single use & nozzles R-1 to R-15 & $6 / 3 / 98$ - single use (exists) \\
\hline nozzles L-1 to L-15 & $10 / 6 / 00-4 / 2 / 06$ & nozzles R-3 to R-9 & now (exists) \\
\hline nozzles L-1 to L-16 & $1 / 9 / 03-1 / 14 / 03$ & nozzles R-14 to R-15 & $4 / 5 / 99-10 / 12 / 99$ \\
\hline nozzles L-2 to L-14 & $1 / 17 / 05-$ single use & nozzles R-14 to R-19 & $1 / 17 / 05-$ single use \\
\hline nozzles L-2 to L-15 & $10 / 10 / 00-4 / 2 / 06$ & nozzles R-15 to R-16 & $10 / 28 / 98-9 / 22 / 99$ \\
\hline nozzles L-2 to L-19 & $7 / 20 / 99-1 / 17 / 05$ & nozzles R-15 to R-19 & $7 / 20 / 99-7 / 1 / 11$ \\
\hline nozzles L-16 to L-19 & $7 / 20 / 99-$ single use & & \\
\hline
\end{tabular}

Additionally, in the 02-A central Pump Pit, Nozzle K shall connect to nozzle $A$ to allow a transfer from $\operatorname{tank} 241-\mathrm{AW}-102$ to $\operatorname{tank} 241$-AW-106 without having the waste go through the 242-A Evaporator. This connection is needed for two transfers scheduled to occur 10/28/98 and 9/22/99.

\section{Electrical System}

Three issues are identified with the electrical system. The first two are derived from previously performed Condition Assessment Survey (CAS) inspections. The issues identified here from the Condition Assessment Survey should be resolved through the corrective maintenance process. Deficiencies documented during the 1995 CAS performance are, however, dated. Establishing baseline information that accurately describes the physical condition of waste transfer system components is important. This can be accomplished via employing the formal DOE CAS methodology or by means of a similar assessment/inspection system. Knowledge of the current condition of equipment/systems is essential to determining reliability, repair, and replacement needs. The third issue deals with the capacity of the electrical system. Work to resolve the third issue is currently underway. 
Table E-3. Electrical System.

\begin{tabular}{|l|l|l|l|}
\hline \multicolumn{1}{|c|}{ Issue } & \multicolumn{1}{|c|}{ Process needs } & \multicolumn{1}{|c|}{ Basis } & \multicolumn{1}{|c|}{$\begin{array}{l}\text { Suggested scope of work } \\
\text { to address issue }\end{array}$} \\
\hline $\begin{array}{l}\text { 1. A 1995-system } \\
\text { assessment survey found } \\
\text { many deficiencies with } \\
\text { MCC-241-AW }\end{array}$ & $\begin{array}{l}\text { Electrical system must be } \\
\text { repaired. }\end{array}$ & $\begin{array}{l}\text { System will be operational } \\
\text { through the end of Phase } \\
\text { 1 Privatization. }\end{array}$ & $\begin{array}{l}\text { Develop a work package } \\
\text { to confirm condition of } \\
\text { MCC-241-AW. } \\
\text { Document specific } \\
\text { components requiring } \\
\text { repair or replacement. }\end{array}$ \\
\hline $\begin{array}{l}\text { 2. 1995 system } \\
\text { assessment for HVAC } \\
\text { identified electrical issues } \\
\text { with the HVAC systems }\end{array}$ & $\begin{array}{l}\text { See Ventilation System } \\
\text { Issue 1. }\end{array}$ & $\begin{array}{l}\text { See Ventilation System } \\
\text { Issue 1. }\end{array}$ & $\begin{array}{l}\text { See Ventilation System } \\
\text { Issue 1. }\end{array}$ \\
\hline $\begin{array}{l}\text { 3. It is not clear that new } \\
\text { loads that will be installed } \\
\text { under project W-211 have } \\
\text { been fully evaluated and } \\
\begin{array}{l}\text { compared with excess } \\
\text { capacity in the system. }\end{array}\end{array}$ & $\begin{array}{l}\text { Upgrade electrical system } \\
\text { TBD }\end{array}$ & $\begin{array}{l}\text { Electrical Transmission } \\
\text { systems may need to be } \\
\text { upgraded to account for } \\
\text { the new loads. }\end{array}$ & $\begin{array}{l}\text { Develop a work package } \\
\text { to review the electrical } \\
\text { system capability and } \\
\text { availability versus use and } \\
\text { document. }\end{array}$ \\
\hline
\end{tabular}

\section{Monitor and Control System}

Two issues are identified with the monitor and control system. The first issue comes from previously performed Condition Assessment Survey (CAS) inspections. The issues identified here from the CAS should be resolved through the corrective maintenance process. Deficiencies documented đuring the 1995 CAS performance are, however, dated. Establishing baseline information that accurately describes the physical condition of waste transfer system components is important. This can be accomplished via employing the formal DOE CAS . methodology or by means of a similar assessment/inspection systern. Knowledge of the current condition of equipment/systems is essential to determining reliability, repair, and replacement needs.

The second issue comes from the fact that some new monitor and control systems are expected to be installed under Projects W-211 and W-314. These instrument systems will need to integrate with existing instrumentation in the Tank Farms. The design work for the new instrumentation is not yet complete. When the design media become available, the integration issue listed here can be addressed. 
HNF-2238

Revision 0

Table E-4. Monitor and Control System.

\begin{tabular}{|l|l|l|l|}
\hline \multicolumn{1}{|c|}{ Issue } & \multicolumn{1}{|c|}{ Process needs } & \multicolumn{1}{|c|}{ Basis } & $\begin{array}{l}\text { Suggested scope of work } \\
\text { to address issue }\end{array}$ \\
\hline $\begin{array}{l}\text { 1. Maintenance issues } \\
\text { were identified in 95 } \\
\text { assessment. }\end{array}$ & $\begin{array}{l}\text { TBD (the primary issue } \\
\text { was the weather tightness } \\
\text { of the enclosures) }\end{array}$ & $\begin{array}{l}\text { WHC-SD-W314-ES-018, } \\
\text { '95 assessment will be } \\
\text { updated to verify } \\
\text { requirements }\end{array}$ & $\begin{array}{l}\text { Develop a work package } \\
\text { to assess the condition of } \\
\text { the 241-AW monitor and } \\
\text { control system. Document } \\
\text { specific components } \\
\text { requiring repair or } \\
\text { replacement. }\end{array}$ \\
\hline $\begin{array}{l}\text { 2. Existing monitor and } \\
\text { control systems need to } \\
\text { interface with new } \\
\text { systems. }\end{array}$ & $\begin{array}{l}\text { Systems need to work in a } \\
\text { coordinated way per the } \\
\text { operations Monitor and } \\
\text { Control }(\mathrm{M} \& C \text { ) concept. }\end{array}$ & $\begin{array}{l}\text { Existing and new } \\
\text { monitoring and control } \\
\text { systems will be operated } \\
\text { by TWRS personnel for } \\
\text { efficiency, all of the farms } \\
\text { should be similar (best } \\
\text { engineering practice). }\end{array}$ & $\begin{array}{l}\text { Specific M\&C integration } \\
\text { requirements will be } \\
\text { developed by Vista } \\
\text { Research }\end{array}$ \\
\hline
\end{tabular}

\section{Utilities Systems}

Two issues associated with the utilities systems were identified. The first issue is the need to provide flush and dilution/dissolution water to the 241-AW Tank Farm. The current system provides only $0.23 \mathrm{~m}^{3} / \mathrm{min}(60 \mathrm{gal} / \mathrm{min})$. A flush rate of $0.23 \mathrm{~m}^{3} / \mathrm{min}(60 \mathrm{gal} / \mathrm{min})$ is likely sufficient to meet all flushing requirements except the waste retrieval transfers from 241-AW-101, but is unlikely sufficient for dilution/dissolution needs.

The other issue is the need to prevent the frequent cycling of the air compressors. Compressed air is used for instrumentation and air lift circulators. The current compressed air receiver tank may be undersized or the system has leaks, causing the compressors to cycle on and off every 30 to 50 seconds. The compressors are experiencing early failures and increased maintenance costs due to the frequent cycling. The reliability of the instrumentation is based on the reliability of the compressed air system. 
HNF-2238

Revision 0

Table E-5. Utilities Systems.

\begin{tabular}{|c|c|c|c|}
\hline Issue & Process needs & Basis & $\begin{array}{l}\text { Suggested scope of work } \\
\text { to address issue }\end{array}$ \\
\hline $\begin{array}{l}\text { 1. Need to provide } \\
\text { water for flush and } \\
\text { dilution/dissolution. }\end{array}$ & $\begin{array}{l}\text { Provide water for } 241-\mathrm{AW} \text { - } \\
101 \text { for dilution/dissolution } \\
\text { and line flushing, and to the } \\
\text { remaining tanks for line } \\
\text { flushing. Line flushing } \\
\text { requirements } 0 \text { to } 100 \% \text { of } \\
\text { flow rate }(0 \text { to } 0.57 \mathrm{~m} / \mathrm{min} \\
{[0 \mathrm{to} 150 \mathrm{gal} / \mathrm{min}] \text {, }} \\
1.83 \mathrm{~m} / \mathrm{sec}[6 \mathrm{ft} / \mathrm{sec}] \text { in } 3-\mathrm{in} \text {. } \\
\text { line) for } 241-\mathrm{AW}-101 \text {. TBD } \\
\mathrm{gal} / \mathrm{min} \text { for remaining tanks. }\end{array}$ & $\begin{array}{l}\text { Tank } 241-\mathrm{AW}-101 \text { is a } \\
\text { source tank for } \\
\text { Envelope } \mathrm{A} \text { and as such } \\
\text { will require } \\
\text { dilution/dissolution water. } \\
\text { Other tanks require line } \\
\text { fiushes following } \\
\text { transfers. Current flush } \\
\text { system capable of } \\
\text { approximately } \\
0.23 \mathrm{~m}^{3} / \mathrm{min}[60 \mathrm{gal} / \mathrm{min} \text { ], } \\
\text { new system to be installed } \\
\text { by W-211 is expected to } \\
\text { provide up to } 0.57 \mathrm{~m}^{3} / \mathrm{min} \\
\text { (150 gal } / \mathrm{min} \text { ). }\end{array}$ & $\begin{array}{l}\text { A defensible basis for the } \\
\text { flowrate will require a } \\
\text { review of solids } \\
\text { resuspension modeis and } \\
\text { potentially pipe loop } \\
\text { pumping studies. A Work } \\
\text { plan and cost estimate for } \\
\text { these activities are } \\
\text { currently being developed } \\
\text { by PNNL. }\end{array}$ \\
\hline $\begin{array}{l}\text { 2. Compressors cycle } \\
\text { every } 30 \text { to } 50 \text { seconds } \\
\text { and experience early } \\
\text { failures. }\end{array}$ & $\begin{array}{l}\text { Determine the cause of the } \\
\text { frequent cycling of the air } \\
\text { compressors, and plan and } \\
\text { execute a solution. }\end{array}$ & $\begin{array}{l}\text { Reliability of some } \\
\text { instrumentation is based } \\
\text { on the reliability of the } \\
\text { compressed air system. } \\
\text { The Compressed Air } \\
\text { system is needed to } \\
\text { support existing and future } \\
\text { operations. }\end{array}$ & $\begin{array}{l}\text { Develop a work package } \\
\text { to assess the compressed } \\
\text { air system and determine } \\
\text { the cause of the frequent } \\
\text { cycling. After the cause } \\
\text { of the cycling has been } \\
\text { determined, a scoping } \\
\text { study of alternatives is } \\
\text { needed to develop a } \\
\text { project requirement. } \\
\text { Alternatives might include } \\
\text { repair or replacement of } \\
\text { buried air lines if leaking } \\
\text { or larger compressed air } \\
\text { receiver tanks. }\end{array}$ \\
\hline
\end{tabular}

\section{Sampling Systems}

No issues were identified with the existing sampling system, the requirements given below attempt to address necessary new capabilities that will be required for the feed delivery system. 
Table E-6. Sampling Systems.

\begin{tabular}{|l|l|l|l|}
\hline \multicolumn{1}{|c|}{ Issue } & \multicolumn{1}{|c|}{ Process needs } & \multicolumn{1}{|c|}{ Basis } & \multicolumn{1}{|c|}{$\begin{array}{l}\text { Suggested scope of work } \\
\text { to address issue }\end{array}$} \\
\hline $\begin{array}{l}\text { 1. No issues were } \\
\text { identified with the current } \\
\text { sampling system. }\end{array}$ & $\begin{array}{l}\text { Any changes to the 241- } \\
\text { AW system shall not } \\
\text { preclude the ability to } \\
\text { obtain grab samples from } \\
\text { two risers in each tank. }\end{array}$ & $\begin{array}{l}\text { Transfers in and out of } \\
\text { DSTs in 241-AW will } \\
\text { require grab samples to } \\
\text { confirm waste } \\
\text { compatibility. Tank 241- } \\
\text { AW-101 may also require } \\
\text { process control grab } \\
\text { samples during waste feed } \\
\text { staging. }\end{array}$ & None \\
\hline 2. & $\begin{array}{l}\text { Provide tank viscosity data } \\
\text { to support transfer } \\
\text { analysis. }\end{array}$ & $\begin{array}{l}\text { Process need, waste } \\
\text { pumpability is a function } \\
\text { of viscosity. Viscosity } \\
\text { data does not exist for all } \\
\text { waste types. }\end{array}$ & None \\
\hline 3. & $\begin{array}{l}\text { Provide "Boil Down" data } \\
\text { to support 242-A } \\
\text { Evaporator operation. }\end{array}$ & $\begin{array}{l}\text { This requirement meets a } \\
\text { 242-A Evaporator process } \\
\text { need. }\end{array}$ & None \\
\hline $\begin{array}{l}\text { 4. Samples will need to } \\
\text { be drawn from 241-AW- } \\
\text { 101 after mixing but } \\
\text { before settling. }\end{array}$ & $\begin{array}{l}\text { Provide sample analysis of } \\
\text { homogenized feed } \\
\text { delivery tanks } \\
\text { (241-AW-101) to support } \\
\text { immobilization operations }\end{array}$ & $\begin{array}{l}\text { Private contract stipulation } \\
\text { of waste constituents. } \\
\text { - }\end{array}$ & None \\
\hline
\end{tabular}

\section{Additional issues associated with tank 241-AW-101}

Beyond the issues identified in the tables above, additional issues are associated with tank 241-AW-101 because it is scheduled to be retrieved and delivered to the private contractors during Phase 1.

The 241-AW-101 tank is a flammable gas watch list tank. The 241-AW-101 tank has a crust of solid material on top of the waste, a layer of liquid waste, and a layer of settled solids or sludge on the tank bottom. Hydrogen and other flammable gasses are continuously generated by the decay processes of the radioactive constituents in the waste. These flammable gasses are trapped by the sludge and crust layers. It is assumed that the tank will require a degassing step before retrieval to control the release of the flammable gasses. The planned degassing process is to gently mix the waste with mixer pumps. There are currently no mixer pumps installed in tank 241-AW-101. A new transfer pump is also needed in tank 241-AW-101 to meet the required flow rate of $0.53 \mathrm{~m}^{3} / \mathrm{min}(140 \mathrm{gal} / \mathrm{min})$.

The waste in tank 241-AW-101 requires dilution to ensure that waste transferred from the tank will not form solids and plug the transfer line during the transfer. The waste also 
HNF-2238

Revision 0

requires dissolution to deliver as much as possible of the tank waste. The requirement for delivery of flush/dilution/dissolution water volume flow is given in the Performance Requirements for Phase 1 Waste feed Delivery Components, HNF-1985 (Claghorn 1998). The flow requirement is to provide from 0 to 100 percent of the delivery flow or up to $0.53 \mathrm{~m}^{3} / \mathrm{min}$ (140 gal $/ \mathrm{min}$ ). The current flush system in the $241-\mathrm{AW}$ Tank Farm can deliver only approximately $0.23 \mathrm{~m}^{3} / \mathrm{min}(60 \mathrm{gal} / \mathrm{min})$. The reason for the low flow rate is that the flush system is delivered through $3.8 \mathrm{~cm}\left(1 \frac{1}{2}-\mathrm{in}\right.$.) pipe. Because the $241-\mathrm{AW}-101$ tank is full of waste, at least initially, the dilution water will need to be added to the pump intake. On the other Envelope A tanks, providing the dilution water through the 2-in. slurry lines is possible. The 2-in. slurry line from the AW-A valve Pit to the 241-AW-101. tank was damaged and can no longer be used. The initial dilution water to be added at the pump intake will need to be delivered to the 01-A central pump pit through an alternate route. As one option, running the flush line overground from the 241-AP Tank Farm flush/dilution/dissolution system and into the $01-\mathrm{A}$ central pump pit may be possible. If an overground line were run, backflow prevention would need to be installed in the 01-A Central Pump Pit.

The waste in tank 241-AW-101 has been evaluated, and addition of caustic to the tank is not beneficial either from a stand point of aided dissolution, or from a standpoint of maintaining the tank within the corrosion concentration limitations (refer to Attachment 5). There may be a benefit from the standpoint of corrosion prevention in the transfer piping to having the capability to flush with inhibited water $(0.01 \mathrm{MNaOH})$.

Noting that the issues listed above will all be addressed by the W-211 Project is important. The 241-AW-101 tank is planned to be included in the scope of project W-211. Definitive design for tank 241-AW-101 is scheduled to begin early in FY 1999. Construction activities are scheduled to be completed by June 2002. As mentioned in Appendix B of this document, it is likely that a reliability benefit would be gained by having the 241-AW-101 tank ready to transfer waste at the beginning of Phase 1.

Table E-7. Additional Tank 241-AW-101 Issues. (3 Sheets)

\begin{tabular}{|c|c|c|c|}
\hline Issue & Process needs & Basis & $\begin{array}{c}\text { Suggested scope of work } \\
\text { to address issue }\end{array}$ \\
\hline $\begin{array}{l}\text { 1. } 241-\mathrm{AW}-101 \text { has no } \\
\text { means to mobilize solids }\end{array}$ & $\begin{array}{l}\text { Install two mixer pumps to } \\
\text { mobilize solids }\end{array}$ & $\begin{array}{l}\text { Decision Document, Phase } \\
\text { I Intermediate Waste Feed } \\
\text { Staging System Design } \\
\text { Requirements (Galbraith } \\
\text { and Daling 1997). Mixer } \\
\text { pumps were the most cost- } \\
\text { effective alternative for } \\
\text { waste mobilization and } \\
\text { mixing }\end{array}$ & none \\
\hline
\end{tabular}




\section{Revision 0}

Table E-7. Additional Tank 241-AW-101 Issues. (3 Sheets)

\begin{tabular}{|c|c|c|c|}
\hline Issue & Process needs & Basis & $\begin{array}{l}\text { Suggested scope of work } \\
\text { to address issue }\end{array}$ \\
\hline $\begin{array}{l}\text { 2. Sodium salts in } \\
241-\mathrm{AW}-101 \text { must be } \\
\text { dissolved prior to delivery } \\
\text { to the privatization } \\
\text { vendor. Saturated sait } \\
\text { solutions can cool and } \\
\text { form precipitates during } \\
\text { transfer. }\end{array}$ & $\begin{array}{l}\text { LAW transfer pump shail } \\
\text { have capability to inject } \\
\text { water or aqueous solutions } \\
\text { of } \mathrm{NaOH} \text { at the pump. } \\
\text { suction bell. }\end{array}$ & $\begin{array}{l}\text { Dilution of waste with } \\
\text { water or dilute caustic } \\
\text { solutions is required to } \\
\text { dissolve solids and prevent } \\
\text { precipitation during } \\
\text { transfer }\end{array}$ & none \\
\hline $\begin{array}{l}\text { 3. Transfer velocity should } \\
\text { be sufficient to avoid line } \\
\text { pluggage. }\end{array}$ & $\begin{array}{l}\text { 241-AW-101 transfer } \\
\text { pump shall deliver waste } \\
\text { feeds at TBD ft/s }\end{array}$ & $\begin{array}{l}\text { Required transfer velocity } \\
\text { is dependent on whether } \\
\text { "supernate" or "slurry" } \\
\text { transfers are being } \\
\text { performed. }\end{array}$ & $\begin{array}{l}\text { The basis for the flowrate } \\
\text { will require that a } \\
\text { definition and flow } \\
\text { requirements for } \\
\text { "supernate" and "slurry" } \\
\text { transfers be developed. } \\
\text { Awaiting work plan and } \\
\text { cost estimate from PNNL. }\end{array}$ \\
\hline $\begin{array}{l}\text { 4. Sodium salts in } \\
241-\mathrm{AW}-101 \text { must be } \\
\text { dissolved prior to delivery } \\
\text { to the privatization } \\
\text { vendor. }\end{array}$ & $\begin{array}{l}\text { System shall have } \\
\text { capability to add up to } \\
3790 \mathrm{~m}^{3}(1,000,000 \mathrm{gal}) \\
\text { water to tank } \\
241-\mathrm{AW}-101 .\end{array}$ & $\begin{array}{l}\text { Preliminary results for } \\
241-A N-105 \text { indicate that } \\
\text { the settled solids will } \\
\text { require approximately a } \\
1: 1 \text { dilution for solids } \\
\text { dissolution. }\end{array}$ & $\begin{array}{l}\text { Complete } 241-\mathrm{AW}-101 \\
\text { flowsheet ESP model runs } \\
\text { to estimate water volume } \\
\text { requirements }\end{array}$ \\
\hline $\begin{array}{l}\text { 5. Dilution water is } \\
\text { needed to dissolve the } \\
\text { waste solids. The mixer } \\
\text { pump intake is near the } \\
\text { tank bottom beneath } 2.7 \mathrm{~m} \\
\text { ( } 9 \mathrm{ft} \text { ) of compact settied } \\
\text { sludge. Start up of the } \\
\text { mixer pump will require } \\
\text { addition of water. }\end{array}$ & $\begin{array}{l}\text { System shall have } \\
\text { capability to add dilution } \\
\text { water to } 241-A W-101 \text { at } \\
\text { or near the mixer pump } \\
\text { intake. The dilution water } \\
\text { capacity shall be } \\
0.27 \mathrm{~m}^{3} / \mathrm{min}(70 \mathrm{gal} / \mathrm{min}) \text {. }\end{array}$ & $\begin{array}{l}\text { Dilution water is needed } \\
\text { near the mixer pump } \\
\text { intakes to provide a } \\
\text { pumpable fluid at the } \\
\text { pump intake for starnup. } \\
\text { Addition to the mixer } \\
\text { pump intake is also the } \\
\text { best way to provide fast, } \\
\text { thorough mixing of the } \\
\text { dilution water with the } \\
\text { waste solids. The dilution } \\
\text { system capacity is } \\
\left.0.53 \mathrm{~m}^{3} / \mathrm{min} \text { (140 gal } / \mathrm{min}\right) \\
\text { added to two mixer } \\
\text { pumps. }\end{array}$ & none \\
\hline $\begin{array}{l}\text { 6. The } 241-A W-101 \\
\text { instrumentation does not } \\
\text { currently provide a means } \\
\text { to accurately locate the } \\
\text { solid/liquid interface as } \\
\text { solids settle. }\end{array}$ & $\begin{array}{l}\text { Provide AW-101 tank } \\
\text { instrumentation capable of } \\
\text { monitoring solids settling } \\
\text { and identifying a } \\
\text { solid/liquid interface in a } \\
\text { tank with setled solids }\end{array}$ & $\begin{array}{l}\text { Monitoring of slurry } \\
\text { density at different depths } \\
\text { is needed to monitor solids } \\
\text { settling and establish the } \\
\text { location of the } \\
\text { liquid/settled solids } \\
\text { interface for controlling } \\
\text { supernate decanting. }\end{array}$ & $\begin{array}{l}\text { It is expected that this } \\
\text { instrumentation will } \\
\text { consist of an ENRAF } \\
\text { gauge with the } \\
\text { densitometer upgrade. } \\
\text { This is the instrument } \\
\text { specified for the W-320 } \\
\text { project. }\end{array}$ \\
\hline
\end{tabular}


HNF-2238

\section{Revision 0}

Table E-7. Additional Tank 241-AW-101 Issues. (3 Sheets)

\begin{tabular}{|c|c|c|c|}
\hline Issue & Process needs & Basis & $\begin{array}{l}\text { Suggested scope of work } \\
\text { to address issue }\end{array}$ \\
\hline $\begin{array}{l}\text { 7. To prolong pump life } \\
\text { the transfer pumps should } \\
\text { be sized to prevent pump } \\
\text { cavitation under planned } \\
\text { operating conditions. } 2\end{array}$ & $\begin{array}{l}241-\mathrm{AW}-101 \text { transfer } \\
\text { pump shall have a NPSHR } \\
\text { of } 5.5 \mathrm{~m}(18 \mathrm{ft}) \text { or less }\end{array}$ & $\begin{array}{l}\text { At } 60^{\circ} \mathrm{C} \text {, no cavitation } \\
\text { should occur at }-43 \mathrm{~cm} \\
(-17 \text { in.) of submergence } \\
\text { (see attachment } 3 \text { ). This } \\
\text { allows a decant pump } \\
\text { (assumed impeller location } \\
68 \mathrm{~cm}(27 \mathrm{in} \text {.) above the } \\
\text { tank bottom) to pump the } \\
\text { tank down to a } 25 \mathrm{~cm} \\
(10 \text { in.) heel. }\end{array}$ & None \\
\hline $\begin{array}{l}\text { 8. A new transfer pump is } \\
\text { required in } 241-\mathrm{AW}-101 \text {. }\end{array}$ & $\begin{array}{l}\text { 241-AW-101 transfer } \\
\text { pump shall have a } \\
\text { maximum developed head } \\
\text { of } 379 \mathrm{ft}(230 \text { psi and } \\
1.41 \mathrm{~g} / \mathrm{ml})\end{array}$ & $\begin{array}{l}\text { The maximum developed } \\
\text { pressure should not exceed } \\
\text { the piping design pressure. }\end{array}$ & $\begin{array}{l}\text { Document logic and } \\
\text { source of design pressure }\end{array}$ \\
\hline $\begin{array}{l}\text { 9. The current retrieval } \\
\text { scenario requires that } \\
\text { clarified supernate be } \\
\text { transferred to the staging } \\
\text { tanks. This will require an } \\
\text { adjustable intake pump to } \\
\text { complete the retrieval. }\end{array}$ & $\begin{array}{l}24 \mathrm{I}-\mathrm{AW}-101 \text { transfer } \\
\text { pump inlet shall be } \\
\text { adjustable from } 10 \mathrm{~m} \text { to } \\
0.25 \mathrm{~m} \text { above the tank } \\
\text { bottom }\end{array}$ & $\begin{array}{l}\text { Best engineering } \\
\text { judgement. } \\
\text { There are alternative } \\
\text { retrieval scenarios which } \\
\text { would utilize } 1 \text { or } 2 \text { fixed } \\
\text { inlet pumps. }\end{array}$ & $\begin{array}{l}\text { The appropriate transfer } \\
\text { pump configuration needs } \\
\text { to be determined. This is } \\
\text { has a number of impacts } \\
\text { on process reliability and } \\
\text { will require an } \\
\text { alternatives evaluation. }\end{array}$ \\
\hline $\begin{array}{l}\text { 10. Addition of cool water } \\
\text { to the waste could result in } \\
\text { precipitation of solids. It is } \\
\text { also desirabie to warm } \\
\text { transfer lines prior to } \\
\text { transferring waste to } \\
\text { reduce potential for solids } \\
\text { precipitation. }\end{array}$ & $\begin{array}{l}\text { Temperature of water } \\
\text { added to tank } 241-\mathrm{AW}-101 \\
\text { and the } 241-\mathrm{AW}-101 \\
\text { transfer pump shall be } \\
\text { adjustable from ambient- } \\
60^{\circ} \mathrm{C}\left(140^{\circ} \mathrm{F}\right) \text { while } \\
\text { delivering the maximum } \\
\text { flowrate (cooling } \\
\text { capability not required). }\end{array}$ & $\begin{array}{l}\text { The maximum allowable } \\
\text { waste temperature is } 90^{\circ} \mathrm{C} \\
\left(195^{\circ} \mathrm{F}\right) \text {. A maximum } \\
\text { temperature of } 60^{\circ} \mathrm{C} \\
\left(140^{\circ} \mathrm{F}\right) \text { allows the } \\
\text { transfer pump to empty } \\
\text { the tank without cavitation } \\
\text { (see Attachment 3). }\end{array}$ & $\begin{array}{l}\text { Provide heating capability } \\
\text { in dilution water system } \\
\text { for tank } 241-\mathrm{AW}-101 \text {. }\end{array}$ \\
\hline $\begin{array}{l}\text { 11. No need was identified } \\
\text { for addition of } \mathrm{NaOH} \text { to } \\
\text { 24l-AW-101. }\end{array}$ & $\begin{array}{l}\text { Remove caustic addition } \\
\text { requirement from W-211 } \\
\text { scope for tank } \\
241-\mathrm{AW}-101 \text {. }\end{array}$ & $\begin{array}{l}\text { There are no plans to add } \\
\text { caustic to the waste in tank } \\
241-\mathrm{AW}-101 \text {. The } \\
241-\mathrm{AW}-101 \text { waste is } \\
\text { within corrosion } \\
\text { specification and will } \\
\text { remain in spec after } \\
\text { addition of dilution water. } \\
\text { See Attachment } 5 \text {. }\end{array}$ & none \\
\hline
\end{tabular}




\section{Revision 0}

Figure 1. AW-A and AW-B Manifold Layout Sketch.

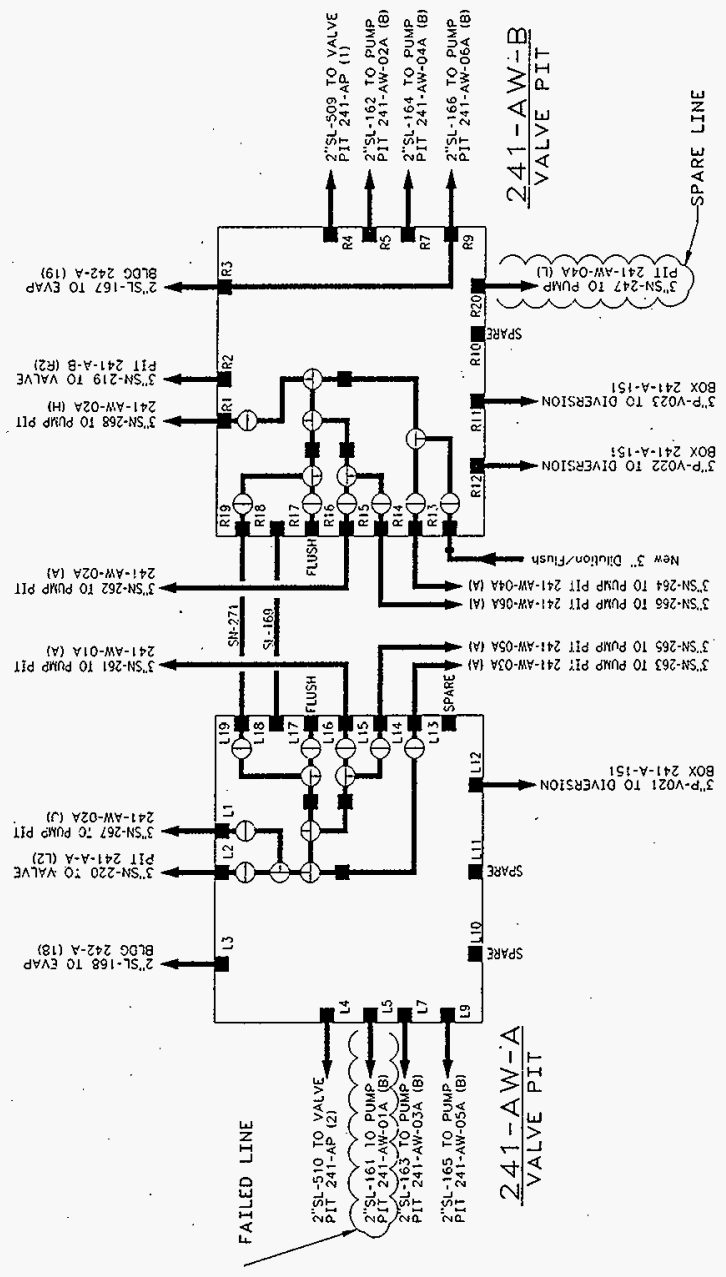




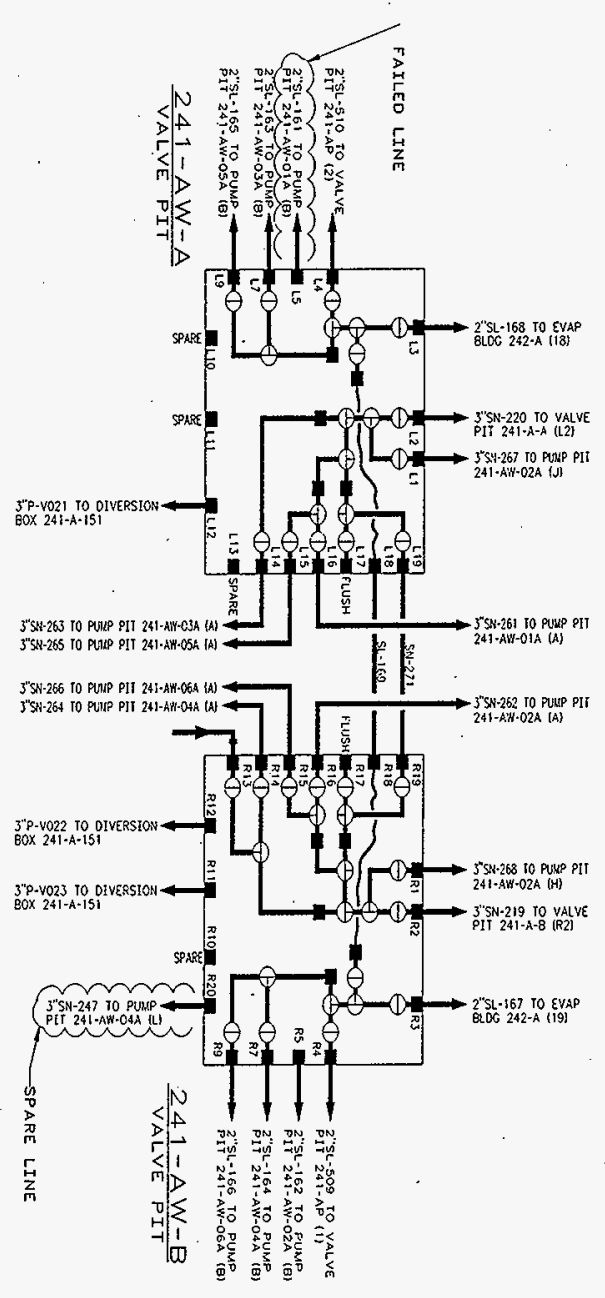

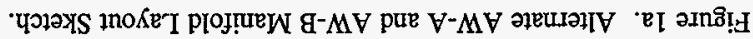


HNF-2238

Revision 0

Figure 2. Tank 241-AW-102 Central Pump Pit Jumper Layout Sketch.

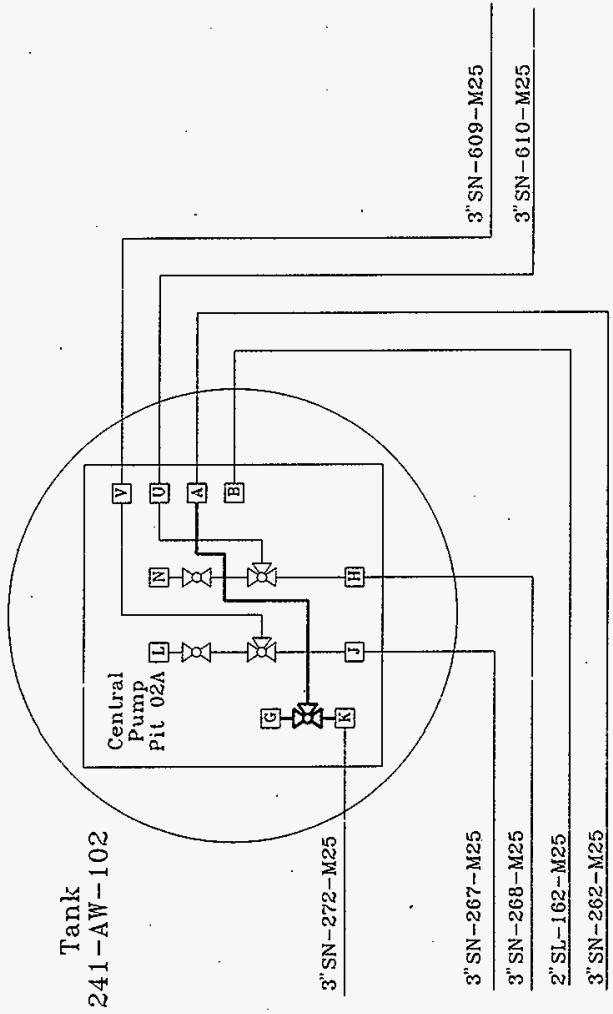


HNF-2238

Revision 0

Figure 3. Central Pump Pit Configuration Sketch.

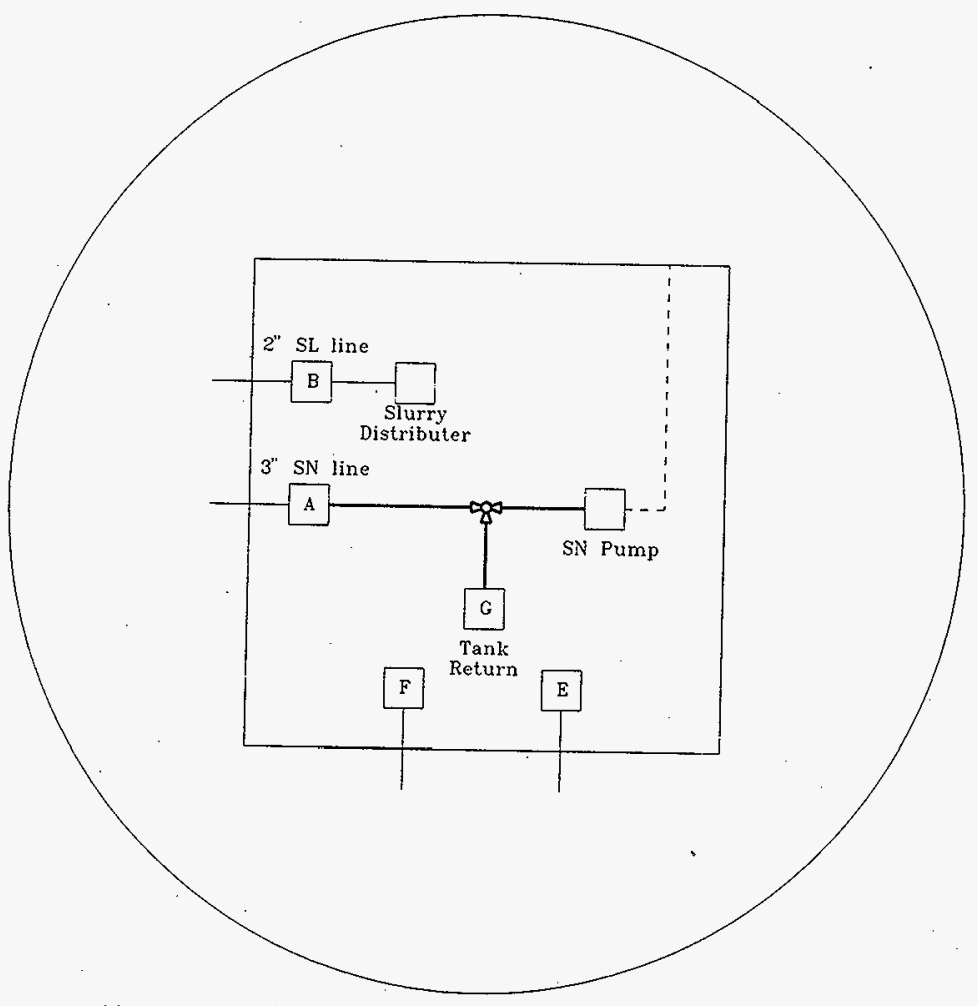


- HNF-2238

Revision 0

Attachment 1 (Sheet 1 of 2)

CALCULATION SHEET

Page $\frac{1}{1}$ of $\frac{1}{0}$

Revision No. 0

Date: May 12.1998

By: BB Peters of

Checked By: wi with who

SUBJECT: Calculation of maximum heatup rate from operation of two mixer pumps

Assumptions: 1)100\% of energy goes to heatup waste

2) each mixer pump is $300 \mathrm{hp}$ (600 hp total)

3) waste heat capacity is same as water ( 1 BTU /16 $\%$ )

4) initial waste temperature is $117{ }^{\circ} \mathrm{F}(47 \mathrm{C})$

- this is maximum temp in AN-104 sludge.

- the average temp in $A \omega-101$ is $-108^{\circ} \mathrm{F}$

5) average density of waste is $1.4 \mathrm{~g} / \mathrm{ml}$

A) Calculate Heat up rate and time to reach. $140^{\circ} \mathrm{F}$ and $195^{\circ} \mathrm{F}$ for a tank with I million gallons

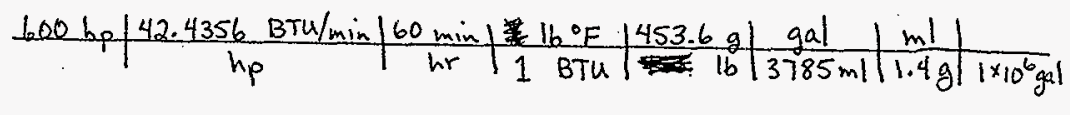

$$
=0.13^{\circ} \mathrm{F} / \mathrm{hr}
$$

$$
\begin{aligned}
& \frac{(140-117)^{\circ} \mathrm{F}}{0.13^{\circ} \mathrm{F} / \mathrm{hr}}=177 \mathrm{hr}(7.4 \text { days }) \text { to reach } 140^{\circ} \mathrm{F} \\
& \frac{(195-117)^{\circ} \mathrm{F}}{0.13^{\circ} \mathrm{F} / \mathrm{hr}}=600 \mathrm{hr}(25 \text { days }) \text { to reach } 195^{\circ} \mathrm{F}
\end{aligned}
$$

E-22

\$112\%8 MOCRHEAT.CAL 


\section{Attachment 1 (Sheet 2 of 2)}

Author: "Onishi; Yasuo" <yasuo.onishi@pni.gov> at EXCHANGE

Date: 4/20/98 1:57 PM

TO: Brian B Peters at HANFORD21E

Subject: RE: Status of DST mixing/waste transfer modeling work

Brian: Message Contents

We completed AP-102 and AP-104 pump jet mixing modeling. We assumed that there will be no yield strength of the disturbed mixture of AN-105 solids and diluents. We selected for test cases; $25 \%$ and $180 \%$ dilution by water with and without solid dissolution effects as starting conditions. For the $25 \%$ dilution case, the mixing was predicted to be very quick, within tens of minutes. For $180 \%$ case, it will take a little over one hour to fully mix the waste. We made these simulation results into video tapes and John Van Beek has a copy. If you are interested in, I will show you the video tape. We may repeat these cases when we get a yield strength value of this disturbed mixture of AN-105 solid and ciluent in the later part of FY98.

We are now setting up our chemical model as a part of the AN-105 pump jet mixing. After confirming our chemical model results, we will conduct pump jet mixing modeling of AN-105 tank with chemical reactions occurring during the mixing. I am assuming that (i) gas is already removed from AN-105 salt cake, and (ii) the supernatant liquid of AN-105 is decanted and the tank was filled back with water, as a starting condition for the AN-105 pump jet mixing.

I was not asked to conduct degas modeling with pump jet mixing, so I assume the degas process will be assessed in some other ways. One way to degas would be to use short bursts of jets to mobilize only a portion of the salt cake at a time to avoid a large gas release. It will take a long time for the solids resuspended during the degas process to settle down so the supcrnate can be decanted, I suspect.

Yasuo 
HNF-2238

Revision 0

Attachment 2 (Sheet 1 of 15)

Requirement Basis

241-AW Primary Ventilation Stack Effluent Monitoring Upgrade

Issue Description:

The 241-AW primary veritilation stack (296-A-27) is currently designated as a minor stack under the site air permit thus it has not been required to meet NESHAP requirements ( $40 \mathrm{CFR} 61$, subpart $\mathrm{H}$ ) for continuous emissions monitoring. The environmental permit groups from both LMH and WMH agree that under a review such as part of a Notice Of Construction (NOC), this stack is likely to be redesignated as a major stack. The current stack would not be compliant with NESHAP monitoring requirements.

The criterion for designation of an effluent discharge stack as minor or major is whether the potential for unabated discharges could result in an off-site exposure of $0.1 \mathrm{mrem} / \mathrm{yr}$. Previous modeling for $241-\mathrm{AW}$ 's current designation as a minor stack identified an exposure potential of $0.04 \mathrm{mrem} / \mathrm{yr}$. Recent and ongoing changes to the air standards would probably revise the current 241-AW primary stack calculations to exceed $0.06 \mathrm{mrem} / \mathrm{yr}$ under current conditions. The consensus among the environmental permitting groups is that the Department of Health is very likely to consider the 241-AW primary stack as a major stack even under the current conditions (safe storage \& evaporator support). Increased activities, such as mixer pump operation and WFD transfers, would provide additional rationale for a major stack designation.

As a major stack, the monitoring requirements will be increased. There are a number of existing projects which could include the scope to upgrade this stack but none appear to have identified it explicitly. Project W-314 will be replacing the entire 241-AW yentilation system during phase 2 of that project. The W-314 CDR shows the schedule for completion of all phase $2 \mathrm{AW}$ farm. upgrades as 12/2003. Tank AW-10I is scheduled for retrieval in 3/2003. Project W-211 will provide for modifications to AW-101, including installation of the mixer pumps, by $7 / 2002$. The NOC for this aspect of Project $W-211$ will likely identify the potential ventilation emissions as exceeding $0.1 \mathrm{mirem} / \mathrm{yr}$.

There is another project (W-420) which is installing fully NESHAP-compliant stacks on the ventilation systems for four DCRTs (244-A, 244-BX, 244-S, 244-TX), 244-CR Vault, 241-SX exhauster, and the exhauster for tanks C-104/C-105/C-106. Initial indications are that the design from this project could be readily applied to $241-\mathrm{AW}$. Based on cost estimates prepared for W-420, installation of a NESHAP-compliant stack at 241 -AW would cost $\$ 100,000$ to $\$ 250,000$.

Project Ownership Issue:

There is a potential ownership issue (WFD/Operations) because current operations (safe storage, evaporator support) in 241-AW may result in the redesignation of the stack without considering impacts from WFD activities. However, upgrades to $241-\mathrm{AW}$ in support of WFD will probably be the actions that trigger a formal redesignation of the stack, and even if ongoing operations did not exceed $0.1 \mathrm{mrem} / \mathrm{yr}$, the additional WFD activities would probably cause this limit to be exceeded, albeit for a temporary period. 
HNF-2238

Revision 0

Attachment 2 (Sheet 2 of 15)

Timecycle/Schedule Issues:

The time required to prepare an NOC and get it approved can take $6-9$ months. Installing, testing and operation of new monitoring equipment could add 3-6 months to the upgrade and compliance process. The earliest need date depends on when the need for review and stack designation is triggered (e.g., by preparation of an NOC for changes to current system design or effluent sources). Project W-314 will be making upgrades to the AW valve pits early next FY. It is not clear whether the NOC for this work would trigger the ventilation system review. The next activity to trigger an NoC review is likely to be project W-211. The W-211 design for tank AW-101 is scheduled to start in 1999 with construction complete by $6 / 2002$.

\section{ATIACHMENTS}

- H-14-020102 Ventilation Tank Primary System (VTP) Stack Monitor P\&ID H-2-74896 296-A-27 Stack Monitor Insiallation

FNE-SD-W420-CDR-001, pages 4-14, Conceptual Design Report for Project W-420, Stack Monitoring Upgrade, Project Description 
HNF-2238

Revision 0

Attachment 2 (Sheet 3 of 15)

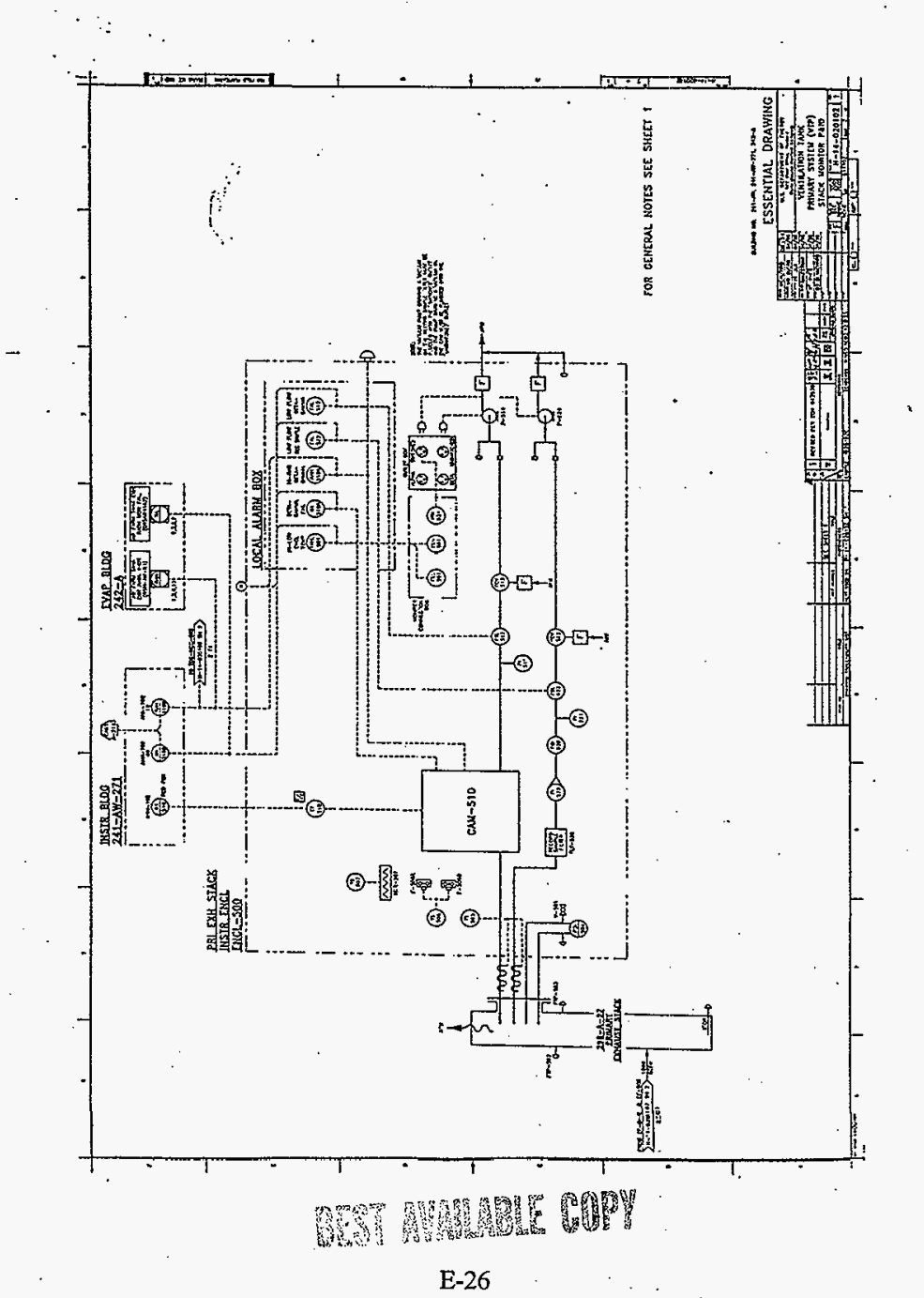


HNF-2238

Revision 0

Attachment 2 (Sheet 4 of 15)

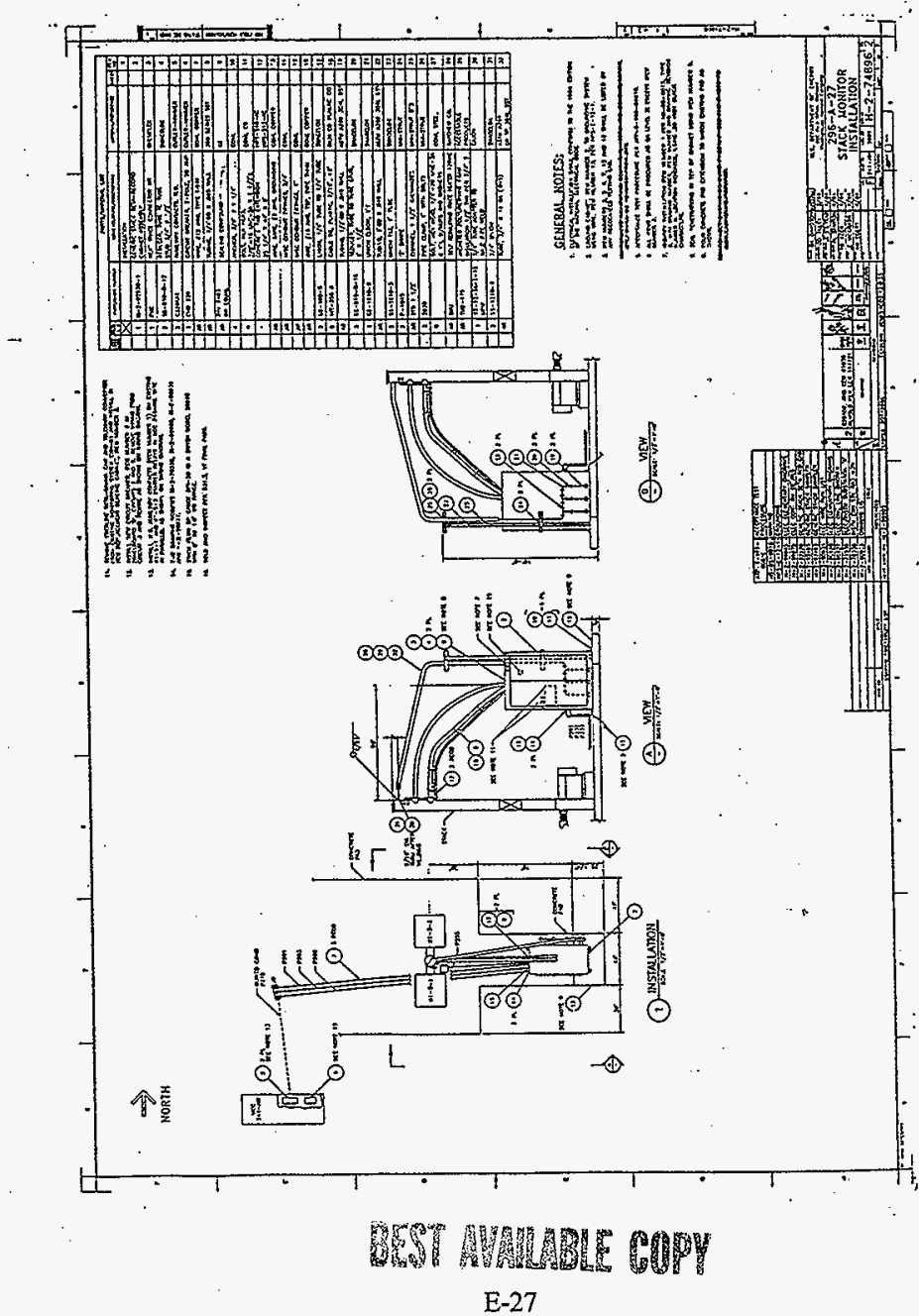


HNF-2238

Revision 0

\section{- Attachment 2 (Sheet 5 of 15)}


HNF-2238

Revision 0

Attachment 2 (Sheet 6 of 15)

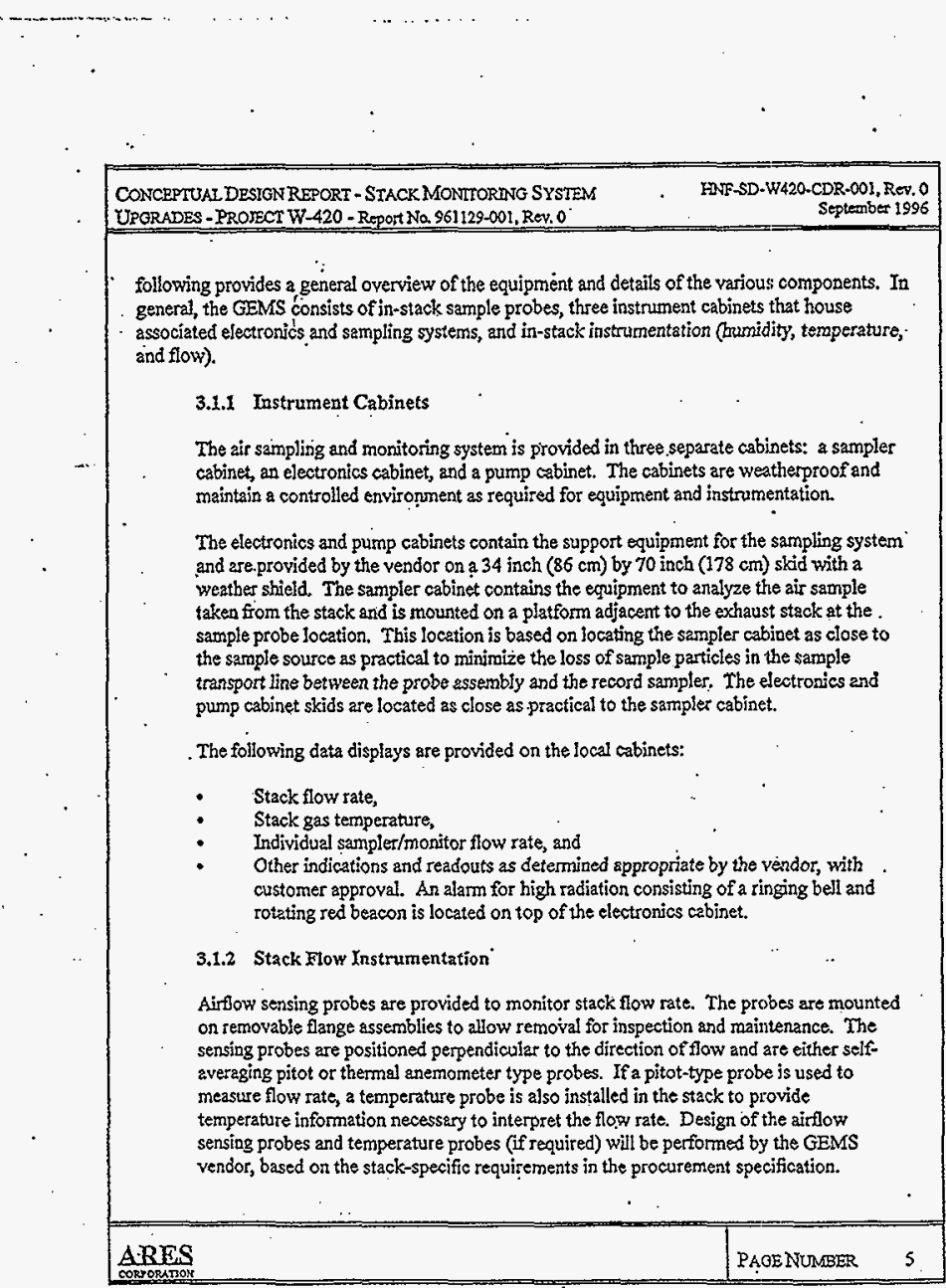


HNF-2238

Revision 0

Attachment 2 (Sheet 7 of 15)

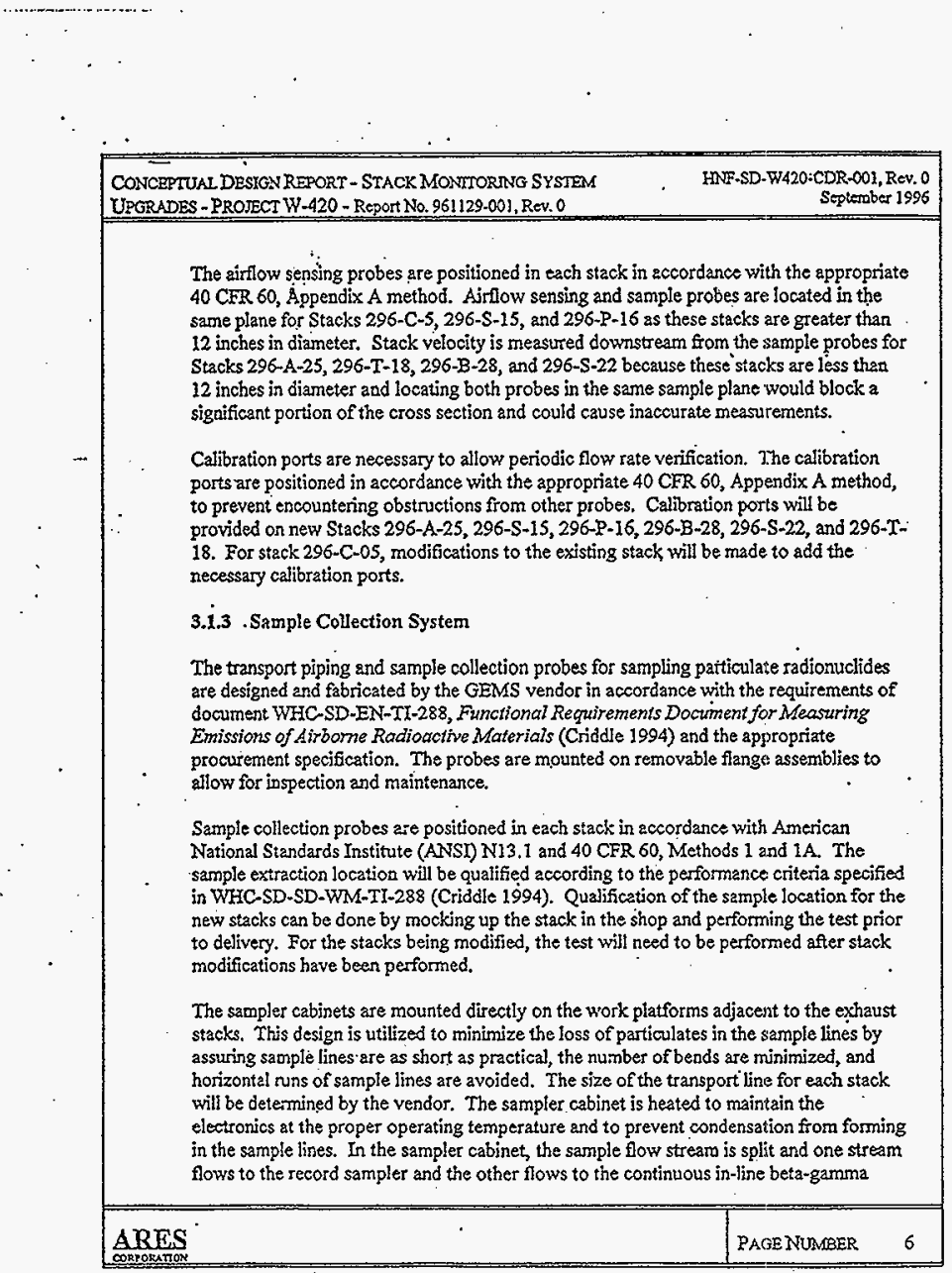


HNF-2238

Revision 0

Attachment 2 (Sheet 8 of 15)

CONCEPTUAL DESTGN REFORT - STACK MONITORING SYSTEM
UTGRADRS - PROTECT W-420 - RepOrTNO.961129-001, Rev, 0
sample head. The flow rate of each sample stream is controlled separately.

The sample transport lines are recombined in the Sampler cabinet and routed to the pump cabinet where the vacuum pumping sources are located. Sample return piping is provided from the pump cabinet to retum the extracted sample to the stack. The size of the return line for each stack will be determined during final design (normally $1 / 2$ inch).

Sample probe, sample transport piping, and sample retum piping will be fabricated from type 316 stainless steel. All tubing is seamless construction with material composition per Americar Society for Testing and Materials (ASTM) A269.

\subsubsection{Particulate Record Sampler}

The particulate record sampler is located in the sampler cabinet. The particulate record sampler collects a sample of particles onto a standard 47 millimeter (mm) membrane filter paper for future radionuclide analysis at a laboratory. A record sampler is required to meet the requirements of 40 CFR 61 Subpart $F$ which requires that "all radionuclides. which could contribute greater than 10 percent of the poteritial effective dose equivalent for a release point shall be measured."

Performance of the particulate record sampler shall be verified and documented by (in order of preference): 1) a field acceptance test, 2) laboratory wind tunnel testing, or 3) the verified model (Criddle 1994). Performance requirements for the record sampler and sample probe are as specified in WHC-S-0400 (White 1995) and WHC-SD-W420-EDC001 (Lott 1996).

\subsubsection{Rarticulate Beta-Gamma Continuous Monitor}

The particulate beta-gamma continuous moritor in-line sample head is located in the sampler cabinet. The counting electronics are located in the electronics cabinet. A local alarm on top of the electronics cabinet is provided for stack high radiation. The alarm includes a ringing bell and rotating red beacon. Auxiliary contacts are provided for tro remote indicators, if desired; one for high radiation and one for continuous air monitor (CAM) failure.

The criteria for the need of a continuous on-line beta-gamma monitor is the graded approach to the application of sampling and monitoring outlined in WHC-SD-WM-II-288 (Cricale 1994). This graded approach is reproduced below in Table 2 where it can be seen that real-time monitoring is required for Potential Effective Dose Equivalents (PEDE) greater than 1.0 mrem/yr.

\begin{tabular}{|l|l|}
\hline ARES & PAGENUMAER 7 \\
\hline
\end{tabular}




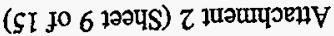


HNF-2238

Revision 0

Attachment 2 (Sheet 10 of 15)

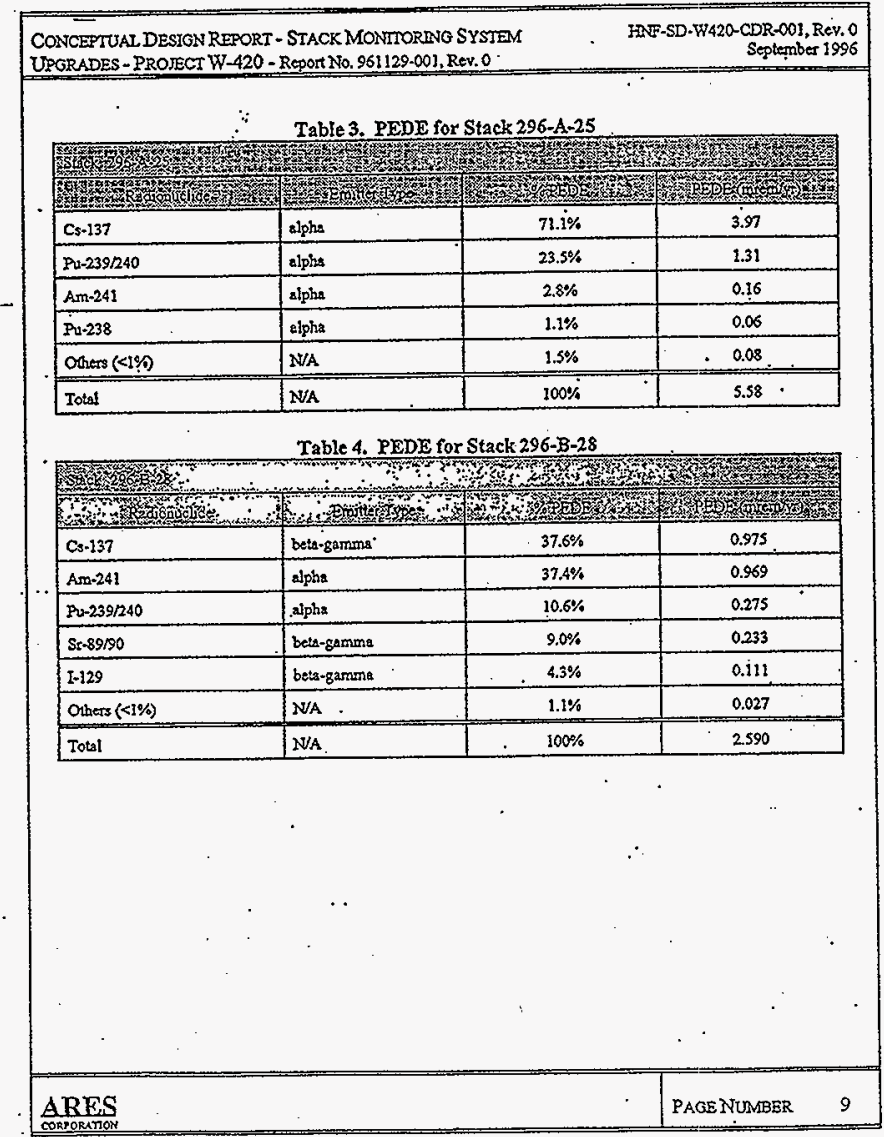


HNF-2238

Revision 0

Attachment 2 (Sheet 11 of 15)

\author{
Attachment 2 (Sheet 11 of 15$)$
}


HNF-2238

Revision 0

Attachment 2 (Sheet 12 of 15)

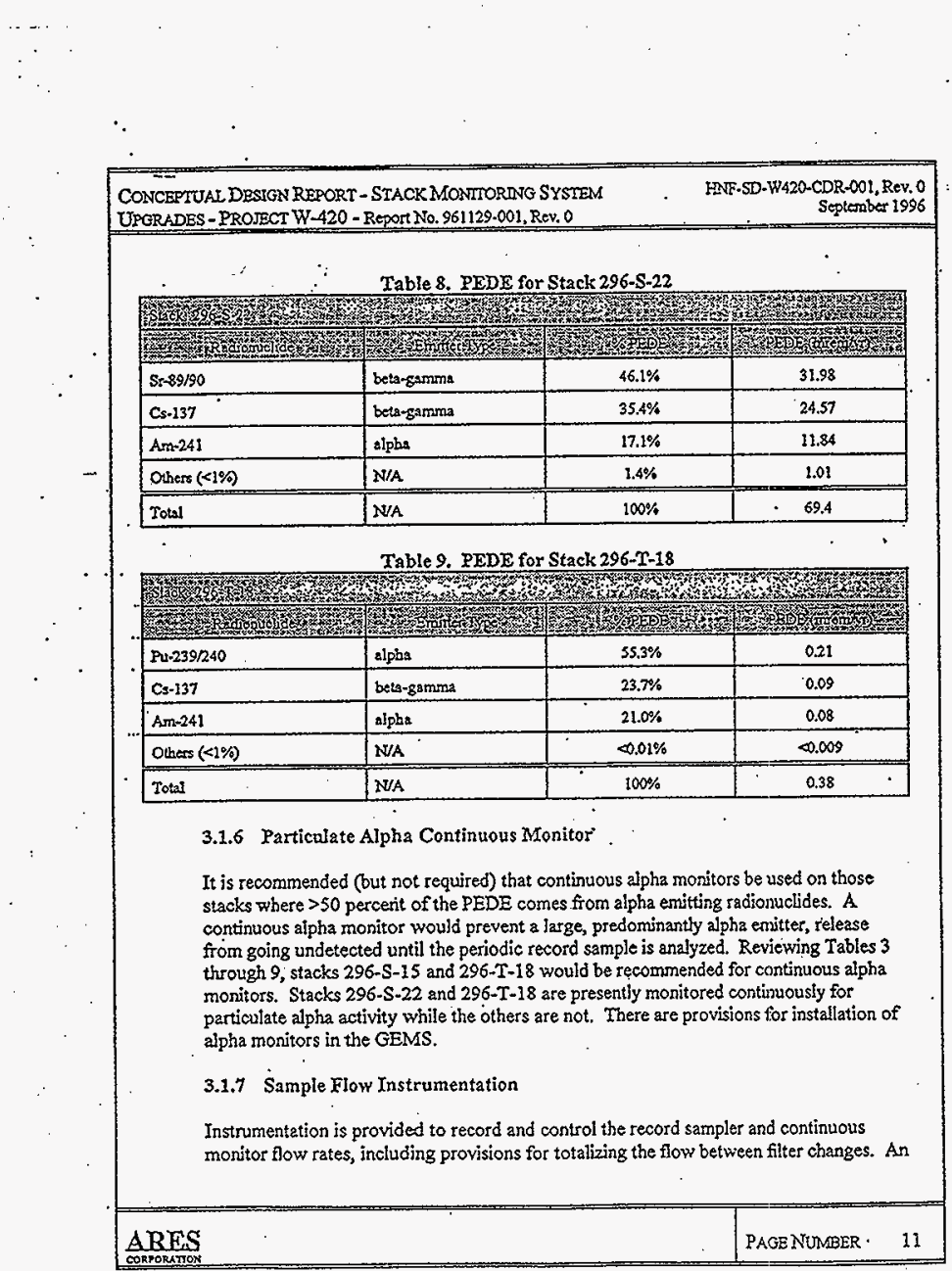


HNF-2238

Revision 0

Attachment 2 (Sheet 13 of 15)

CONCEPTUAL DESTGN REPORT - STACK MONTTORRNG SYSTEM

UPGRADES - PROJECT W-420 - Report No. 961129 -001, Rev. 0

HNF-SD-W420-CDR-001, Rev. 0 Septerber 1996

adjustable alarm set point for sample low flow rate is provided. Auxiliary contacts are - provided for femote indication, if desired, when sample flow rate deviates beyond required tolerances.

\subsubsection{Data Collection System}

The Data Collection System shall have a standardjzed communications interface with the existing host computer for the transfer, on command, of recorded values.

The Data Collection System shall record and store stack flow rate, stack semperature, stack relative humidity, record sampler flow rate, total stack flow, and total record sampler flow since the last filter change-out. The beta-gamma moritor shall have its flow rate and readings stored periodically. The data storage shall have a method of data transfer for archival purposes. The transfer media shall be PC compatible.

The Data Collection System shall have a method of transferring data during filter changeout. The record sampler flow readings and total flow, the stack llow readings and total flow, and the date and time of the starting and ending data points shall be the minimum information transferred. The transfer media shall be PC compatible.

\subsubsection{Yacuum System}

A vacuurn system is located in the pump cabinet. The system provides a steady, nonpulsing, vacuum source over a range of operating conditions from the maximum and minimum design values of the samplers based on the required collection efficiency. Two vacuum pumps are provided for redundancy.

Each particulate sampling line has its own flow regulation. The vacuum supply to each sample line is adjustable and automatically regulated so that individual sample flow rates are maintained. Components can be isolated individually by isolation valves provided.

The individual vacuum exhaust lines are combined in to a single return manifold. The combined return manifold.is connected by field piping to the stack downstream of sampling and velocity probes.

\subsection{Stacks}

An analysis was performed to determine the ability of the existing stacks to accept the required probes. The decision to use an existing stack configuration or to replace or modify a stack is based on the ability to provide satisfactory locations for sample and flow sensing probes. The

\begin{tabular}{|l|l|}
\hline ARES & PAGENUABER 12 \\
\hline
\end{tabular}


HNF-2238

Revision 0

\title{
Attachment 2 (Sheet 14 of 15)
}

\author{
COACEPTUAL DESIGN REPORT - STACK MONTORMTE SYSTEM \\ UPGRADES:-PROJECT W-420-REOOCTNO. $961129-001 ;$ REv. 0
}

IDF.SD-W420-CDR-O1, REv. 0 September 1996

locations for the sample extraction and flow sensing probes have been chosen in accordance with ANSI N13.1, the appropriate 40 CFR 60 Appendix A method, and WHC-SD-EN-TI-288 (Criddle 1994). An existing stack is used when the probe mounting location(s) satisfy the applicable criteria. New stack extensions, modifications to existing stacks, or replacement stacks are specified when the existing probe locations do not satisfy the applicable requirements. Appropriate modifications are described for each stack.

The locations for flow sensing probes were chosen to satisfy the requirements of the appropirate 40 CFR 60 method, Method 1 for stacks greater than 12 inches in diarneter, and Method $1 \mathrm{~A}$ for stacks less than 12 inches in diameter. Following installation in the field, an initial stack flow sensor verification test will be performed in accordance with 40 CFR 52 Appendix $\mathrm{E}$. When practical, flow sensing probes sre located in the same plane as the sample probes.

The locations for sample probes were chosen in accordance with the guidance of ANSIN13.1 and the requirements of the appropriate $40 \mathrm{CFR} 60$ Appendix A method. In general, these locations must not exhibit angular or cyclonic flow and must provide acceptable uniformity of contaminant mixing. Specific performance criteria for sample extraction locations is provided by FHC-SDEN-TI-288 (Criddle 1994).

Testing (shop or field) will be performed to qualify the sample extraction locations according to the performance criteria of WHC-SD-EN-TI-288 (Criddle 1994). The velocity profile at the sample location will be measured in accordance with 40 CFR 60 Appendix A, Method 1, Section 2.4, "Verification of the Absence of Cyclonic Flow." Satisfactory contaminant mixing will be "demonstrated in accordance with the methods described in WHC-SD-EN-TI-288 (Criddle 1994), . Section 3.3, "Methods for Qualifying the Sample Extraction Location."

An assumption has been made that locating sample probes in accordance with the ANSI N13.1 and $40 \mathrm{CFR} 60$ Appendix A will result in sariple locations that satisfy performance criteriz of WHC-SD-EN-II-288 (Criddle 1994). The validity of this assumption will be demonstrated by shop or field testing. When the existing sample locations are in accordance with ANSI N13.1 and $40 \mathrm{CFR} 60$ Appendix A, the sample location will be qualified by field testing after performance of any additional stack modifications.

Several of the existing stack designs utilize flow straightening vanes because the stacks have tangential inlets which tend to induce swirling. An assumption has been made that flow straightening vanes will not be required with the new stack extensions. However, removable spool pieces are provided to allow installation of flow vanes if testing indicates unacceptable flow conditions at the sample extraction location.

As mentioned previously, the decision to feplace or modity a stack is based on the ability of the 
HNF-2238

Revision 0

Attachment 2 (Sheet 15 of 15)

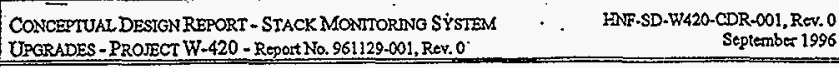

stack to.provide satisfactory probe locations. An assumption has been made that the existing stack heights provide adequate dilution and dispersion. It was not within the scope of Conceptual Design for Project W- 420 to verify that existing stacks satisfy requirements for dilution. and dispersion of contanuinants. Therefore, the original stack height is used as the minimum height for new stack extensions. This should be confirmed early in Definitive Design or in a separate study.

. Preliminary calculations indicate that several of the existing stacks have low stack discharge velocity that may result in extensive downwash along the stack. It is recommended that a separate study be performed prior to detailed design of new stack extensions to ensure the stack effluent escapes the air flow patterns surrounding adjacent buildings and provides adequate dilution and dispersion of contaninants to prevent an unacceptable situation when the efflusent reaches the ground,

\subsection{Vtilities .}

Electrical power for the stack monitors will be provided from the same parel boards that supply the existing stack monitors. Existing conduit will be utilized to the maximum extent practical. All electrical materials and equipment shall be UL or FM tested, with labels attached, for the purpose intended, whenever such products are available. Installation methods shall be in accordance with manufacturer's instructions, NFPA 70 , and with other applicable requirements. A 30 A, 120 VAC, single phase power supply is required for the stack monitors. The single feed connects with a power distribution pasel in the pump cabinet supplied by the stack moritor vendor. Distribution from the pump cabinet to the sample cabinet and the electronics cabinet will be performed through short nuns of galvanized rigid steel (or flexible seal tight) conduit.

\subsection{Work Platforms}

Work platforms are provided for routine access to the sample cabinets which are located next to the sampic probe location. The work platforms shall have guardrails and toe boards that conform to 29 CFR 1910.23(c), and ladders that conform to 29 CFR 1910:27. Conceptual Design allows for a 60 degree pitch for the ladders. A safety chain will be provided at the access to the platform. 
HNF-2238

Revision 0

Attachment 3 (Sheet 1 of 6 )

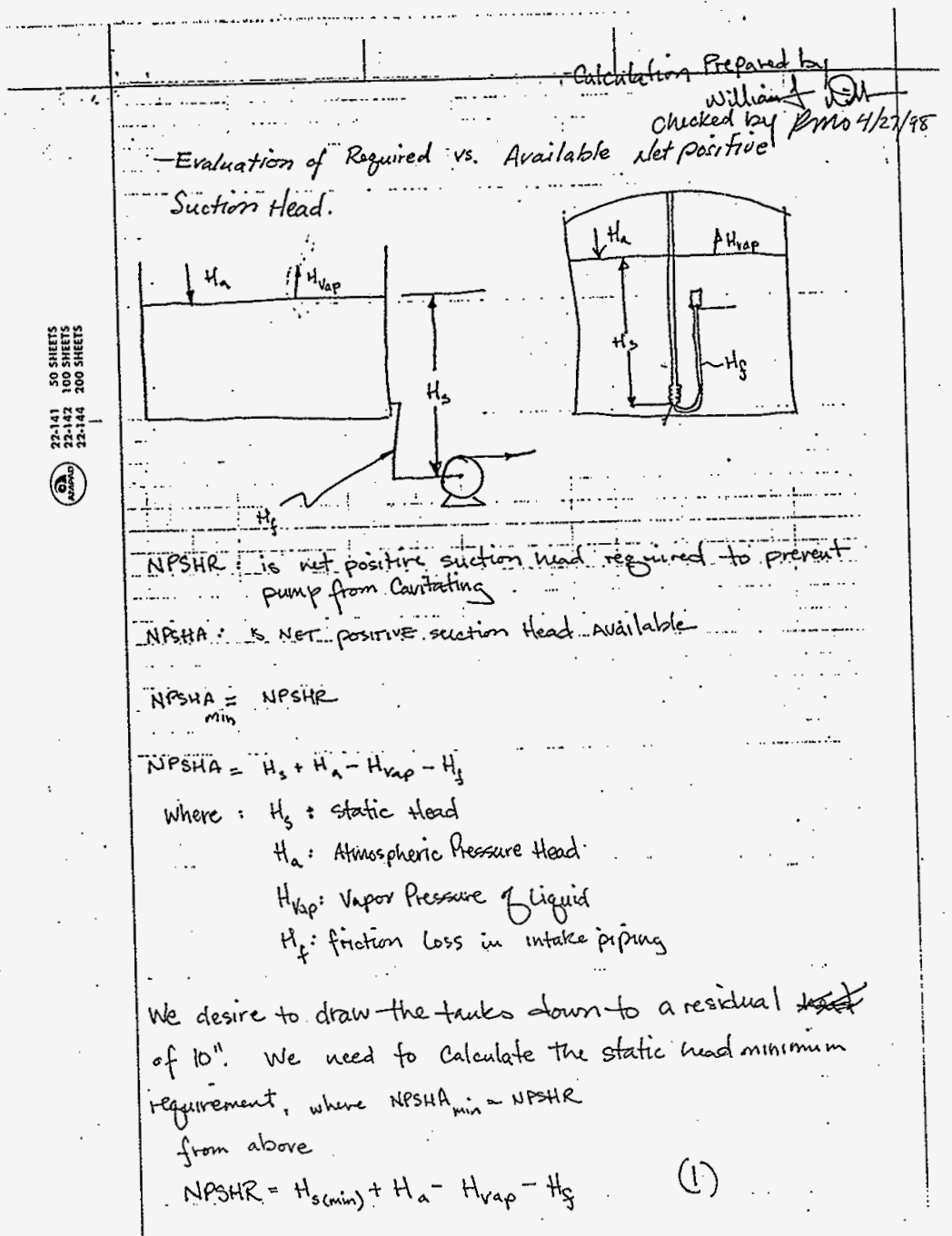

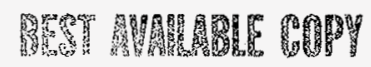

E-39 
HNF-2238

Revision 0

Attachment 3 (Sheet 2 of 6 )

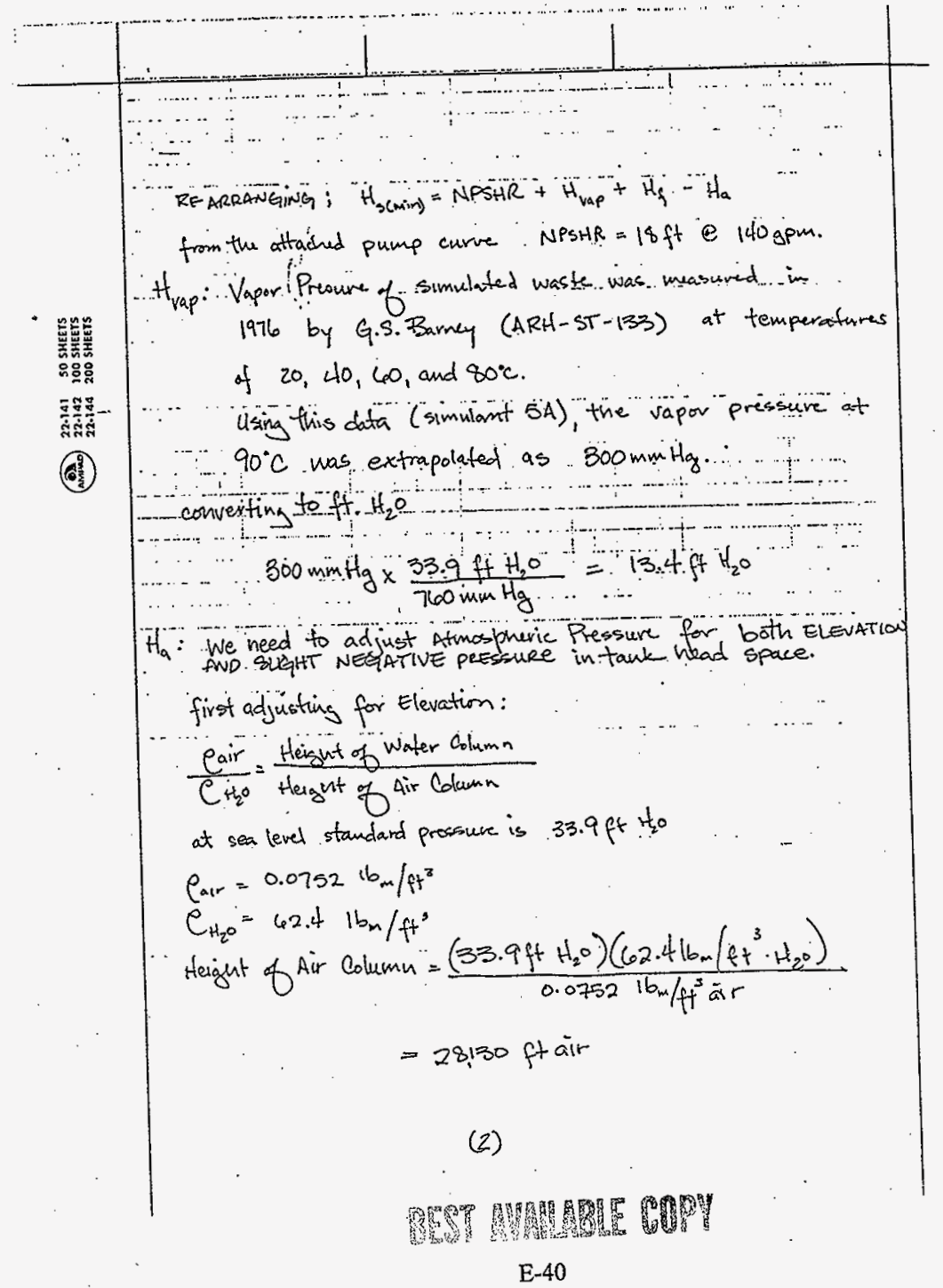


HNF-2238

Revision 0

Attachment 3 (Sheet 3 of 6 )

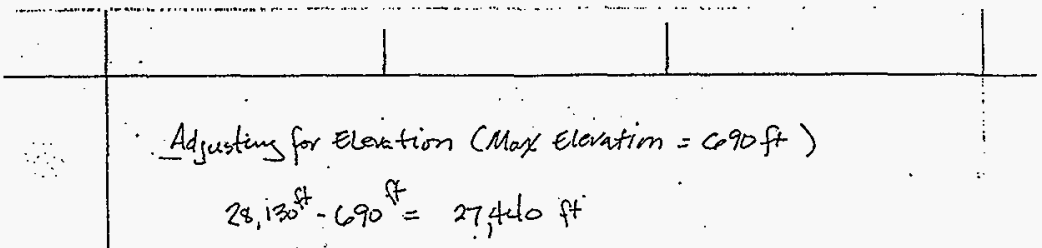

Converting ito $\mathrm{ft}_{2} \mathrm{O}$

$$
27,440 \mathrm{ftair} \times \frac{33.9 \mathrm{ft} \mathrm{H}_{2} \mathrm{O}}{28,130 \mathrm{ft} \text { Air }}=33.1 \mathrm{ft} \mathrm{H}_{2} \mathrm{O}
$$

Adjusting for Negative tank pressure. Assume task pressure is - 4 in $W G$

$$
\begin{aligned}
H_{A} & =33 . F+4 i m \\
& =32.7 \mathrm{f}+\mathrm{H}_{2}{ }^{\circ}
\end{aligned}
$$

$H_{f}$ Per Senior Fiexonics (Manufactures of flex hire) frictional loss in flex is $\approx 7$ finds that in

From flow of Fluid: Through Valves, Fittings, and Pipe." The pressuRe drop is 1.99 psillooft for three inch pipe at a flow tate of $140 \mathrm{gpm}$.

Since the flexible suction hose is $20 \mathrm{ft} \times 3$ in. stainless steel flex hose the Head loss is estimated

$$
\begin{aligned}
& \text { as } \frac{1.99 \rho s i}{100 \mathrm{ft}} \times 20 \mathrm{ft} \times 7 \times \frac{33.9 \mathrm{psift} \mathrm{H}_{2} \mathrm{O}}{14.7 \mathrm{ps} i} \\
& =6.4 \mathrm{ff} \mathrm{H}_{2} \mathrm{O}
\end{aligned}
$$

Hence

$$
\begin{aligned}
H_{\text {SIm) })} & =18 \mathrm{ft} \mathrm{H}_{2} \mathrm{O}+13.4 \mathrm{ft} \mathrm{H}_{2} \mathrm{O}+6.4 \mathrm{ft} \mathrm{H}_{2} \mathrm{O}-32.7 \mathrm{ft} \mathrm{H}_{2} \mathrm{O} \\
& =5.1 \mathrm{ft}
\end{aligned}
$$

(3)

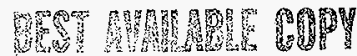

E-41 
HNF-2238

Revision 0

Attachment 3 (Sheet 4 of 6)

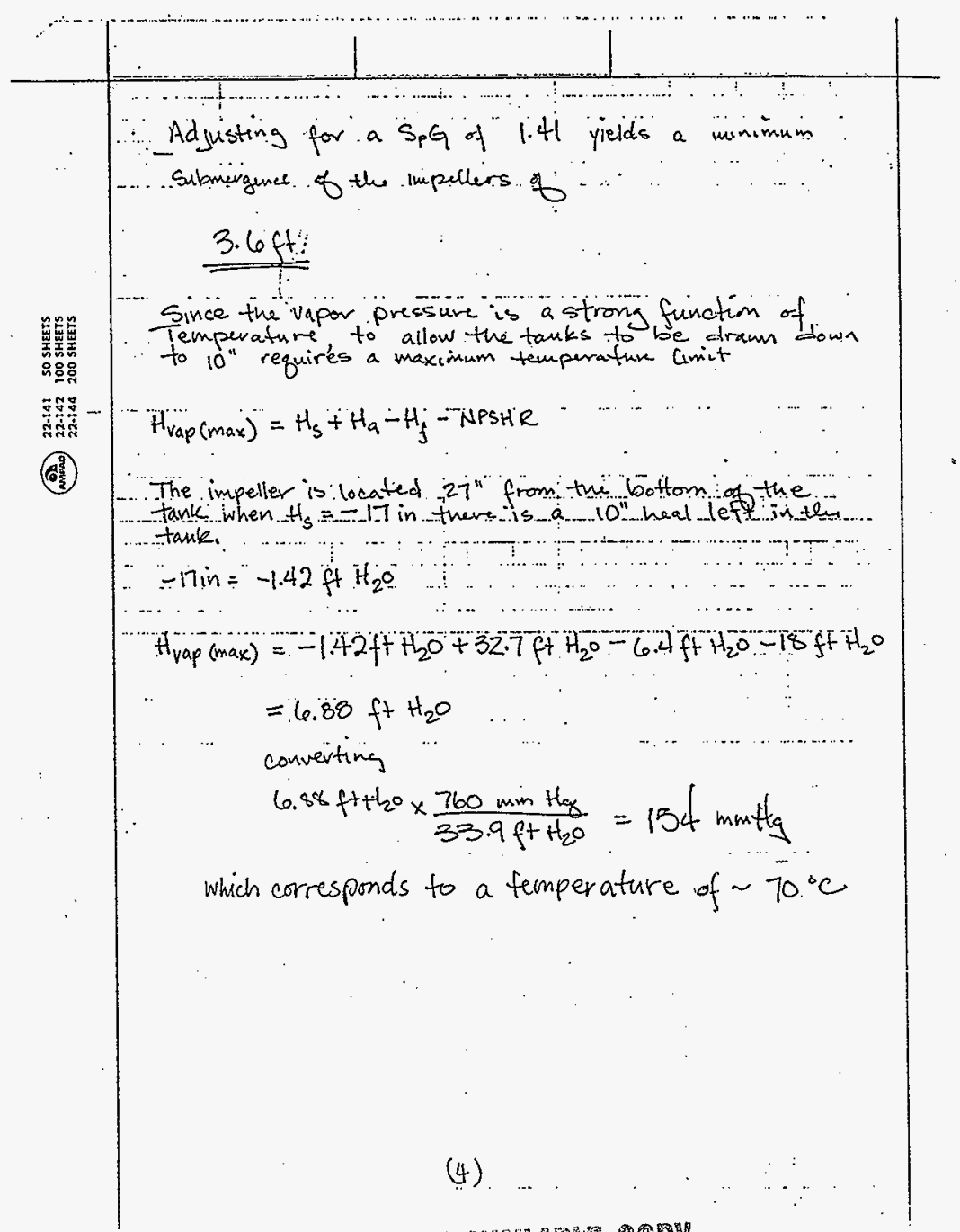


HNF-2238

Revision 0

Attachment 3 (Sheet 5 of 6)

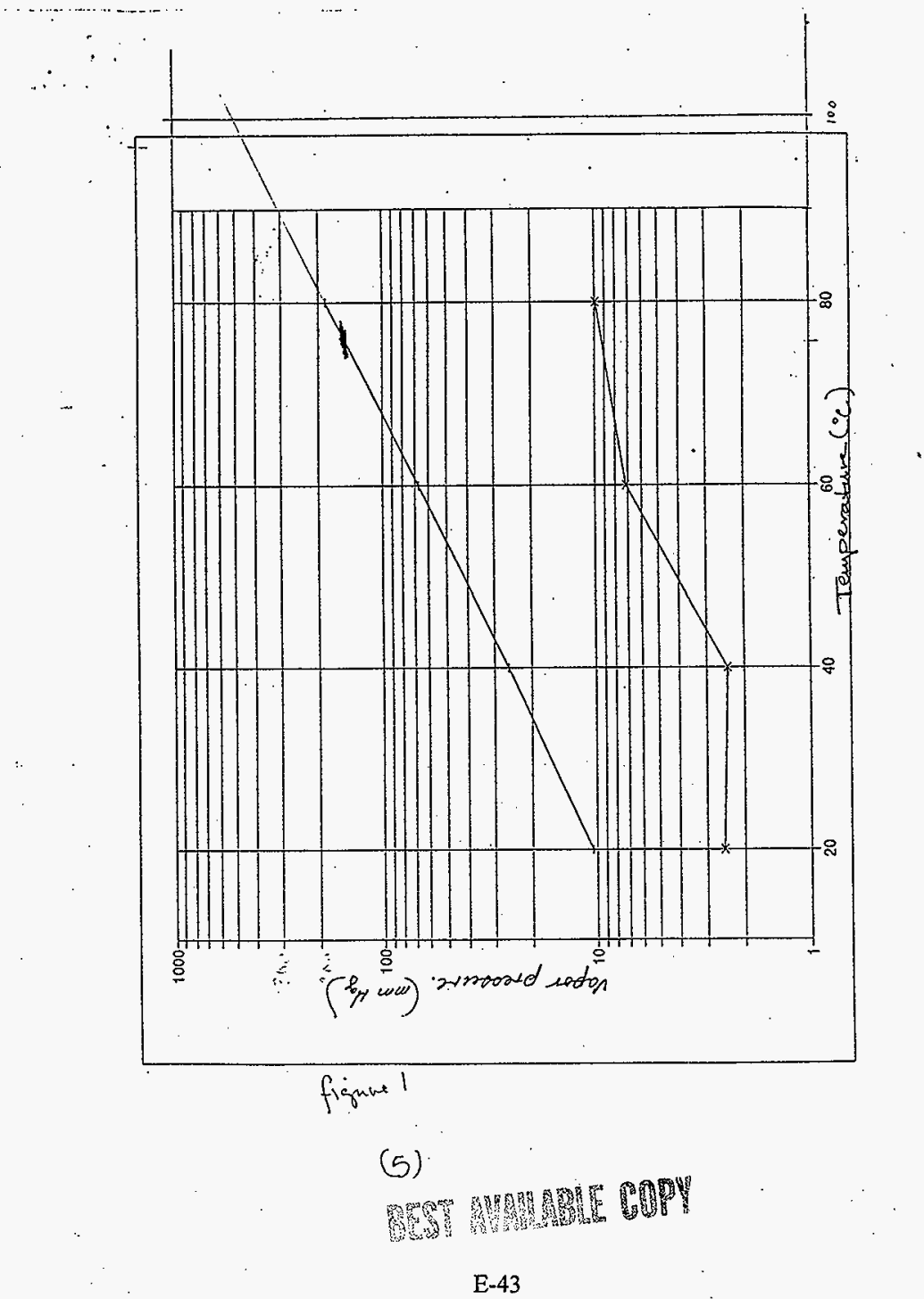


HNF-2238

Revision 0

\section{Attachment 3 (Sheet 6 of 6 )}

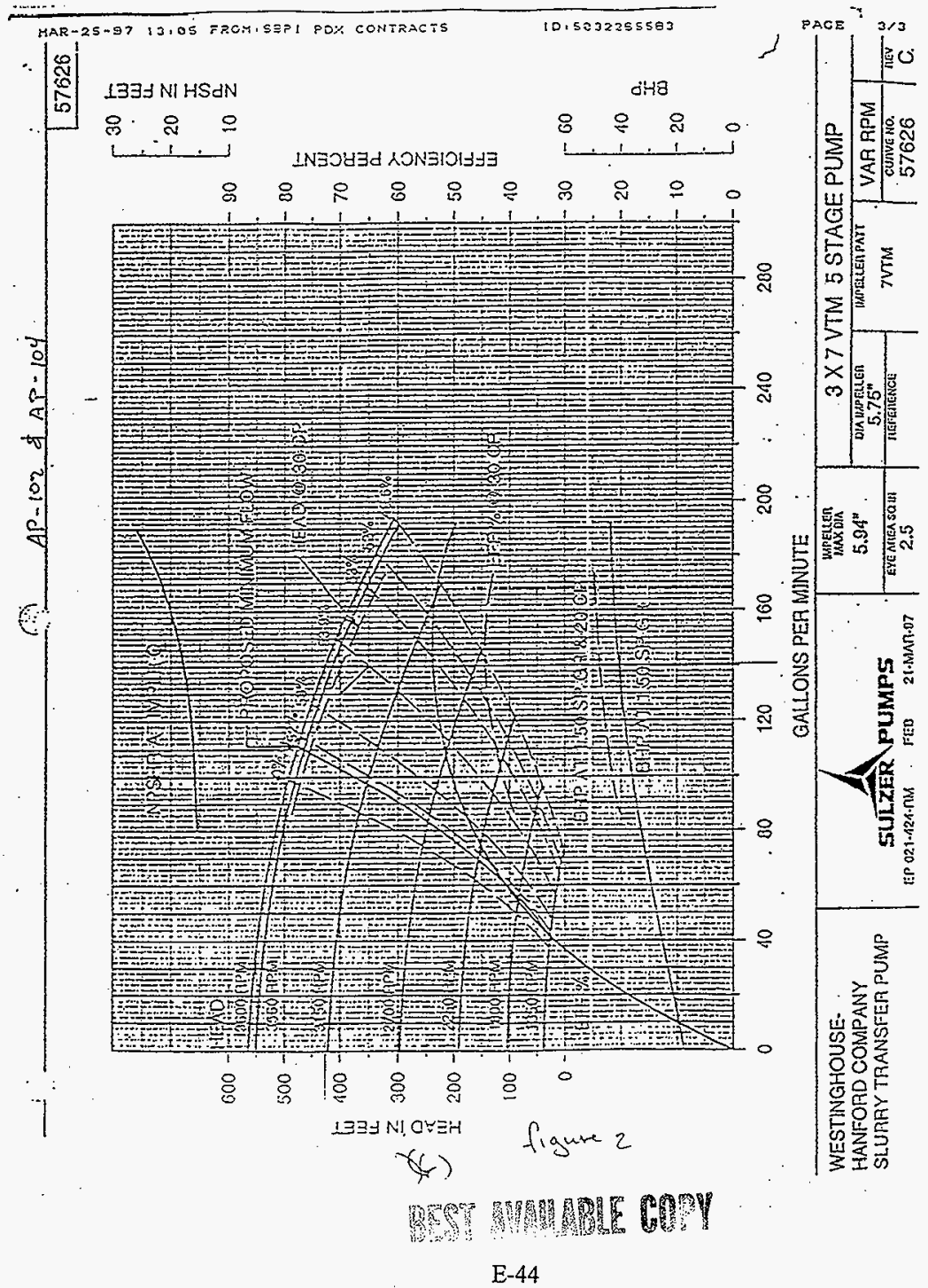


HNF-2238

Revision 0

CALCULATTON SHEET Attachment 4 (Sheet 1 of 1 )

Page $\frac{1}{\text { Revision No. }} \frac{1}{0}$

Revision No. $\frac{0}{390}$

Date:_May 12.1998.

By: BB Peters.

Checked By:Wh whls

SUBJECT: Calculation of Maximum Head for AW-104 Pump

Assumptions: 17 maximum waste density is $1.4 \mathrm{~g} / \mathrm{m} 1$.

2) Line operating pressure is 230 psi

3) Set maximum head equal to line operating pressure

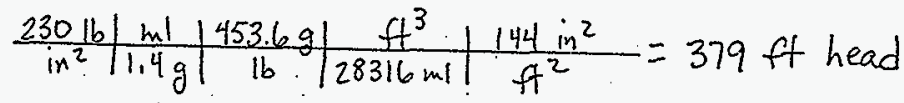


HNF-2238

Revision 0

This page intentionally left blank. 


$$
\begin{aligned}
& \bar{W} S S>\quad[\stackrel{\varepsilon}{\varepsilon} \mathrm{ON}] \\
& \bar{W}: 1<\quad[\mathrm{Z} \mathrm{ON}]+[\mathrm{HO}]
\end{aligned}
$$

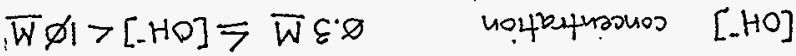

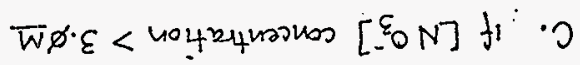

$$
\left(\left[-\varepsilon^{\varepsilon} \mathrm{ON}\right]\right)+\varnothing<\quad[-\mathrm{ON}]+[. \mathrm{HO}]
$$

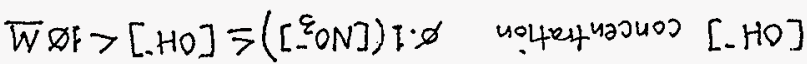

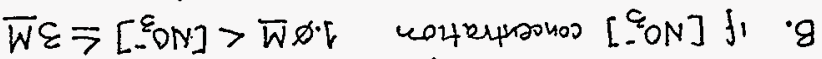

$$
S \cdot \tau>\left(\left[\left[^{2} O N\right]+[. H O]\right) /\left[E_{-} O N\right]\right.
$$

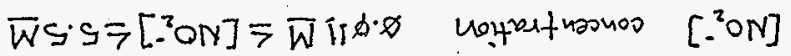

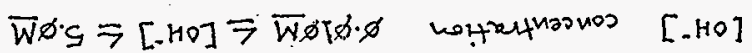

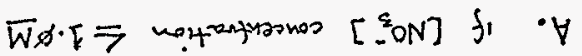

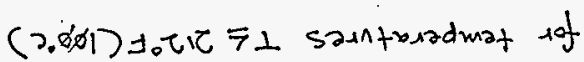

$$
\begin{aligned}
& \text { noyirifioads vorseniog 'I }
\end{aligned}
$$

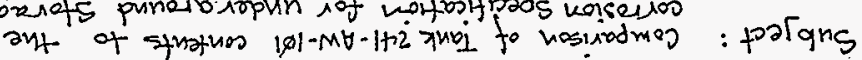

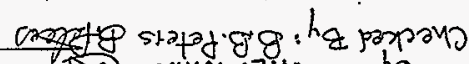

fin silm $7 \mathrm{M} M: \mathrm{h}_{\mathrm{g}}$

sbbl 'bl how

$\varepsilon$ to $f$ stod

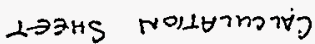


HNF-2238

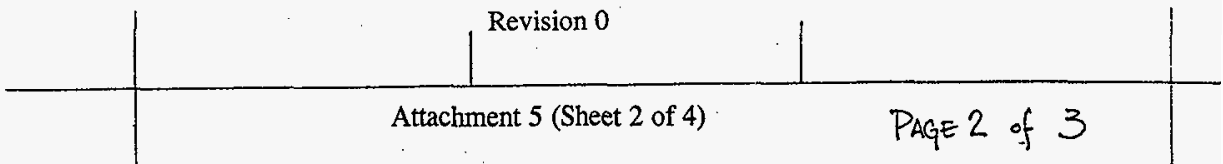

Comparison of Tank 241-AW- $|\varnothing|$ contents to the Corrosion Specification for undergrad storage tanks. (continued)

II. The water survey for Tank $241-A W-|\Phi|$ in Appendix D lists Kilogram quantities of 10 hic species for the waste. Undiluted waste and waste diluted with. $25,5 \dot{\phi}$, and $75 \%$ are included in the water survey.

A. Undiluted Waste - volume $=68.057 \mathrm{~L} / \mathrm{hr}$.

$$
\mathrm{OH}^{-}=3.7738 \mathrm{~kg} / \mathrm{hr}, \mathrm{NO}_{2}^{-}=6.3220 \mathrm{~kg} / \mathrm{hr}, \mathrm{NO}_{3}^{-}=13.389 \mathrm{~kg} / \mathrm{hr}
$$

converting to molarity $(\mathrm{Mol} / \mathrm{L})$

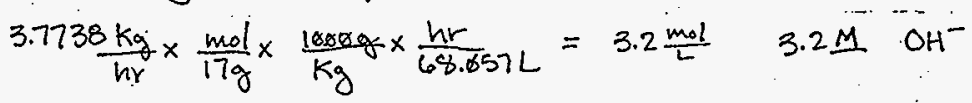$$
6.322 \phi \frac{\mathrm{kg}}{\mathrm{hr}} \times \frac{\mathrm{mol}}{46 \mathrm{~g}} \times \frac{6 \phi \phi g \mathrm{~g}}{\mathrm{~kg}} \times \frac{\mathrm{hr}}{68 . \phi 57 \mathrm{C}}=2.1 \mathrm{M} \mathrm{NO}_{2}^{-}
$$$$
13.389 \frac{\mathrm{kg}}{\mathrm{hr}} \times \frac{\mathrm{mol}}{62 \mathrm{~g}} \times \frac{1 \% 60 \mathrm{gg}}{\mathrm{kg}} \times \frac{\mathrm{hr}}{68657^{2}}=3.2 \mathrm{M} \mathrm{N \textrm {NO } _ { 3 } ^ { - }}
$$

Condition $C$ is met with undiluted waste

B. $25 \%$ dilution - volume $=84.623 \mathrm{~L} / \mathrm{hr}$

$$
\mathrm{OH}^{-}=3.7797 \mathrm{~kg} / \mathrm{hr}, \mathrm{NO}_{2}^{-}=6.322 \varnothing \mathrm{kg} / \mathrm{hr}, \mathrm{NO}_{3}^{-}=13.367 \mathrm{~kg} / \mathrm{hr}
$$

converting to molairty

$$
\begin{aligned}
& 3.7797 \frac{\mathrm{kg}}{\mathrm{hr}} \times \frac{\mathrm{mol}}{17 \mathrm{~g}} \times \frac{1 \phi \phi g \mathrm{~g}}{\mathrm{~kg}} \times \frac{\mathrm{hr}}{84.623 \mathrm{~L}}=2.6 \mathrm{M} \mathrm{OH} \\
& 6.322 \phi \frac{\mathrm{kg}}{\mathrm{hr}} \times \frac{\mathrm{mol}}{46 \mathrm{~g}} \times \frac{\mathrm{6066}}{\mathrm{kg}} \times \frac{\mathrm{hr}}{84.623 \mathrm{~L}}=1.6 \mathrm{M} \mathrm{NO}_{2}^{-} \\
& 13.367 \frac{\mathrm{kgg}}{\mathrm{hr}} \times \frac{\mathrm{mol}}{62 \mathrm{~g}} \times \frac{10 p \phi \mathrm{g}}{\mathrm{kg}} \times \frac{\mathrm{hr}}{84.623 \mathrm{~L}}=2.5 \mathrm{M} \mathrm{NO}_{3}^{-}
\end{aligned}
$$

Condition $B$ is met with waste diluted by $25 \%$

E-48 
HNF-2238

Revision 0

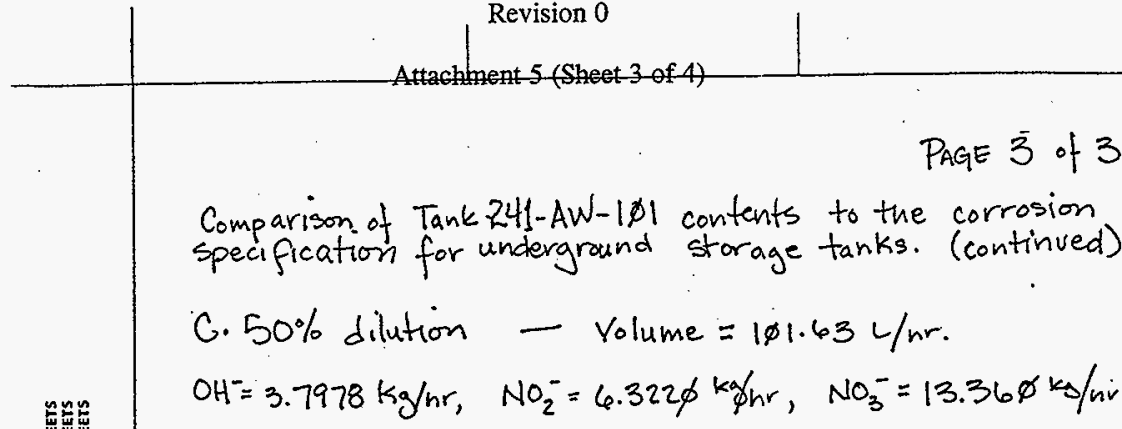

converting to marty

$$
\begin{aligned}
& 3.7978 \frac{\mathrm{kg}}{\mathrm{hr}} \times \frac{\mathrm{mol}}{17 \mathrm{~g}} \times \frac{1898 \mathrm{gg}}{\mathrm{kg}} \times \frac{\mathrm{hr}}{101.63 \mathrm{~L}}=2.2 \mathrm{M} \mathrm{\textrm {OH } ^ { - }} \\
& 6.3220 \frac{\mathrm{kg}}{\mathrm{hr}} \times \frac{\mathrm{md}}{46 \mathrm{~g}} \times \frac{106 \mathrm{gg}}{\mathrm{kg}} \times \frac{\mathrm{hr}}{101.63 \mathrm{~L}}=1.4 \mathrm{M} \mathrm{NO}_{2}^{-} \\
& 13.36 \phi \frac{\mathrm{kg}}{\mathrm{hr}} \times \frac{\mathrm{mol}}{62 \mathrm{~g}} \times \frac{1 \phi \phi \phi g}{\mathrm{~kg}} \times \frac{\mathrm{hr}}{1 \phi 1.63 \mathrm{~L}}=2.1 \mathrm{M} \mathrm{N \textrm {NO } _ { 3 } ^ { - }}
\end{aligned}
$$

Condition $B$ is met with waste diluted by $50 \%$

D. $75 \%$ dilution - volume $=118.92 \mathrm{~L} / \mathrm{hr}$.

converting to molarity $\mathrm{OH}^{-}=3.9039 \frac{\mathrm{kg}}{\mathrm{hr}}, \mathrm{NO}_{2}=6.322 \phi \frac{\mathrm{kag}}{\mathrm{hr}}, \mathrm{NO}_{3}^{\circ}=13.359 \frac{\mathrm{ks}}{\mathrm{hr}}$

$$
\begin{aligned}
& 3.8039 \frac{\mathrm{kg}}{\mathrm{hr}} \times \frac{\mathrm{mol}}{17 \mathrm{~g}} \times \frac{1 \phi \phi \phi g}{\mathrm{~kg}} \times \frac{\mathrm{hr}}{118.92 \mathrm{~L}}=1.9 \mathrm{M} \mathrm{OH} \\
& 6.322 \frac{\mathrm{kg}}{\mathrm{hr}} \times \frac{\mathrm{mol}}{46 \mathrm{~g}} \times \frac{1 \phi \phi g}{\mathrm{~kg}} \times \frac{\mathrm{hr}}{118.92 \mathrm{~L}}=1.2 \mathrm{M} \mathrm{NO}- \\
& 13.359 \frac{\mathrm{kg}}{\mathrm{hr}} \times \frac{\mathrm{mol}}{62 \mathrm{~g}} \times \frac{1 \phi \phi \phi g}{\mathrm{~kg}} \times \frac{\mathrm{hr}}{118.92 \mathrm{~L}}=1.8 \mathrm{M} \mathrm{NO}-
\end{aligned}
$$

Condition 3 is met with waste diluted by $75 \%$

E-49 
HNF-2238

Revision 0

Attachment 5 (Sheet 4 of 4)

CONIROILIDD COPY

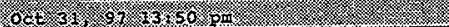

\subsection{UNDERGROUND STORAGE TANKS SPECIFICATTION}

\subsubsection{TANK COMPOSITION}

7.2.1.A Temperatures $\left(T \leq 212^{\circ} \mathrm{F}\right)$

Variable

For $\left[\mathrm{NO}_{3}\right] \leq 1.0 \mathrm{M}:$

[OH]

$\left[\mathrm{NO}_{2}{ }^{\mathrm{T}}\right]$

. $\left[\mathrm{NO}_{3}\right] /\left([\mathrm{OH}]+\left[\mathrm{NO}_{2}\right]\right)$
Specification Limit

$0.010 \mathrm{M} \leq\left[\mathrm{OH}^{-}\right] \leq 5.0 \mathrm{M}$

$0.011 \mathrm{M} \leq\left[\mathrm{NO}_{2}\right] \leq 5.5 \mathrm{M}$

$<2.5$

(for solutions below $167^{\circ} \mathrm{F}$, the [OH'] limit is $8.0 \mathrm{M}$ )

For $1.0 \mathrm{M}<\left[\mathrm{NO}_{3}\right] \leq 3.0 \mathrm{M}$ :

$$
\begin{array}{ll}
{[\mathrm{OH}]} & 0.1\left(\left[\mathrm{NO}_{3}\right]\right) \leq[\mathrm{OH}]<10 \mathrm{M} \\
{\left[\mathrm{OH}^{-}\right]+\left[\mathrm{NO}_{2}^{-}\right]} & \geq 0.4\left(\mathrm{NO}_{3}^{-1]}\right)
\end{array}
$$

For $\left[\mathrm{NO}_{3}{ }^{-}\right]>3.0 \mathrm{M}$ :

$$
\begin{aligned}
& {[\mathrm{OH}]} \\
& {\left[\mathrm{OH}^{-}\right]+\left[\mathrm{NO}_{3}^{\cdot}\right]} \\
& {\left[\mathrm{NO}_{3}^{-}\right]}
\end{aligned}
$$

$$
\geq 1.2 \mathrm{M} \leq .3 \mathrm{M} \leq[\mathrm{OH}]<10 \mathrm{M}
$$

7.2.1.B For High Operating Temperatures $\left(T>212^{\circ} \mathrm{F}\right.$ for $A Y$ and $A Z$ tapks) - section 7.2.1.A temperature limits apply with the exception that $\mathrm{OH}^{\circ}$ concentration must be $<4 \underline{\mathrm{M}}$.

No\%

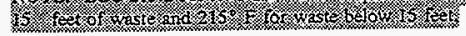

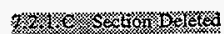

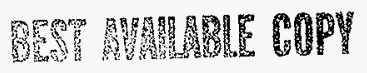




\section{DISTRIBUTION SHEET}

\begin{tabular}{|c|c|c|c|c|c|}
\hline \multirow{2}{*}{$\begin{array}{l}\text { To } \\
\text { Distribution }\end{array}$} & \multirow{2}{*}{\multicolumn{3}{|c|}{$\begin{array}{l}\text { From } \\
\text { TWRS Process Development }\end{array}$}} & \multicolumn{2}{|c|}{ Page 1 of 1} \\
\hline & & & & \multicolumn{2}{|c|}{ Date $5 / 22 / 98$} \\
\hline \multicolumn{4}{|l|}{ Project Title/Work Order } & \multicolumn{2}{|c|}{ EDT No. 622714} \\
\hline \multicolumn{4}{|c|}{$\begin{array}{l}\text { Evaluation of Tank Waste Transfers at 241-AW Tank Farm, } \\
\text { HNF-2238, Rev. } 0\end{array}$} & N No. $\quad N / A$ & \\
\hline Name & MSIN & $\begin{array}{c}\text { Text } \\
\text { With All } \\
\text { Attach. }\end{array}$ & Text Only & $\begin{array}{l}\text { Attach./ } \\
\text { Appendix } \\
\text { Only }\end{array}$ & $\begin{array}{l}\text { EDT/ECN } \\
\text { Only }\end{array}$ \\
\hline $\begin{array}{l}\text { Central Files } \\
\text { DIMC } \\
\text { DOE Reading Room }\end{array}$ & $\begin{array}{l}B 1-07 \\
\mathrm{H} 6-15 \\
\mathrm{H} 2-53\end{array}$ & $\begin{array}{l}x \\
x \\
x\end{array}$ & & & \\
\hline $\begin{array}{l}\text { J. N. Alibert } \\
\text { P. R. Angel ier } \\
\text { D. G. Baide } \\
\text { S. K. Baker } \\
\text { G. E. Binghan } \\
\text { K. A. Boes } \\
\text { T. W. Bohan } \\
\text { H. L. Boston } \\
\text { D. E. Bowers } \\
\text { A. B. Carlson } \\
\text { K. G. Carothers } \\
\text { R. D. Claghorn } \\
\text { G. L. Crawford } \\
\text { T. W. Crawford } \\
\text { R. A. Dodd } \\
\text { M. F. Erhart } \\
\text { B. G. Erlandson } \\
\text { J. W. Ficklin } \\
\text { J. D. Galbraith } \\
\text { J. S. Garfield } \\
\text { C. E. Hatch } \\
\text { D. M. Hammond } \\
\text { J. L. Homan } \\
\text { D. P. Kerwick } \\
\text { N. W. Kirch } \\
\text { J. R. Kriskovich } \\
\text { D. L. Lamberd } \\
\text { C. E. Leach } \\
\text { M. W. Manderbach } \\
\text { R. P. Marshall } \\
\text { W. C. Miller } \\
\text { K. E. Myers } \\
\text { D. R. Nunamaker } \\
\text { R. M. Orme } \\
\text { I. G. Papp (4 copies) } \\
\text { W. A. Peiffer } \\
\text { B. B. Peters } \\
\text { R. D. Potter } \\
\text { W. I. Powell } \\
\text { O. R. Rasmussen } \\
\text { D. W. Reberger } \\
\text { M. A. Rickenbach } \\
\text { C. A. Rieck } \\
\text { S. H. Rifaey } \\
\text { T. W. Staehr } \\
\text { W. T. Thompson } \\
\text { J. E. VanBeek } \\
\text { T. L. Haldo II } \\
\text { K. A. White } \\
\text { W. L. Willis (10 copies) }\end{array}$ & 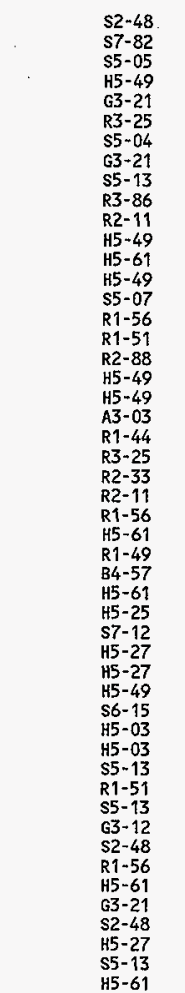 & $\begin{array}{l}x \\
x \\
x \\
x \\
x \\
x \\
x \\
x \\
x \\
x \\
x \\
x \\
x \\
x \\
x \\
x \\
x \\
x \\
x \\
x \\
x \\
x \\
x \\
x \\
x \\
x \\
x \\
x \\
x \\
x \\
x \\
x \\
x \\
x \\
x \\
x \\
x \\
x \\
x \\
x \\
x \\
x \\
x \\
x \\
x \\
x \\
x \\
x \\
x \\
x \\
x\end{array}$ & & . & \\
\hline
\end{tabular}

